\title{
NIST Center for Neutron Research Accomplishments and Opportunities
}
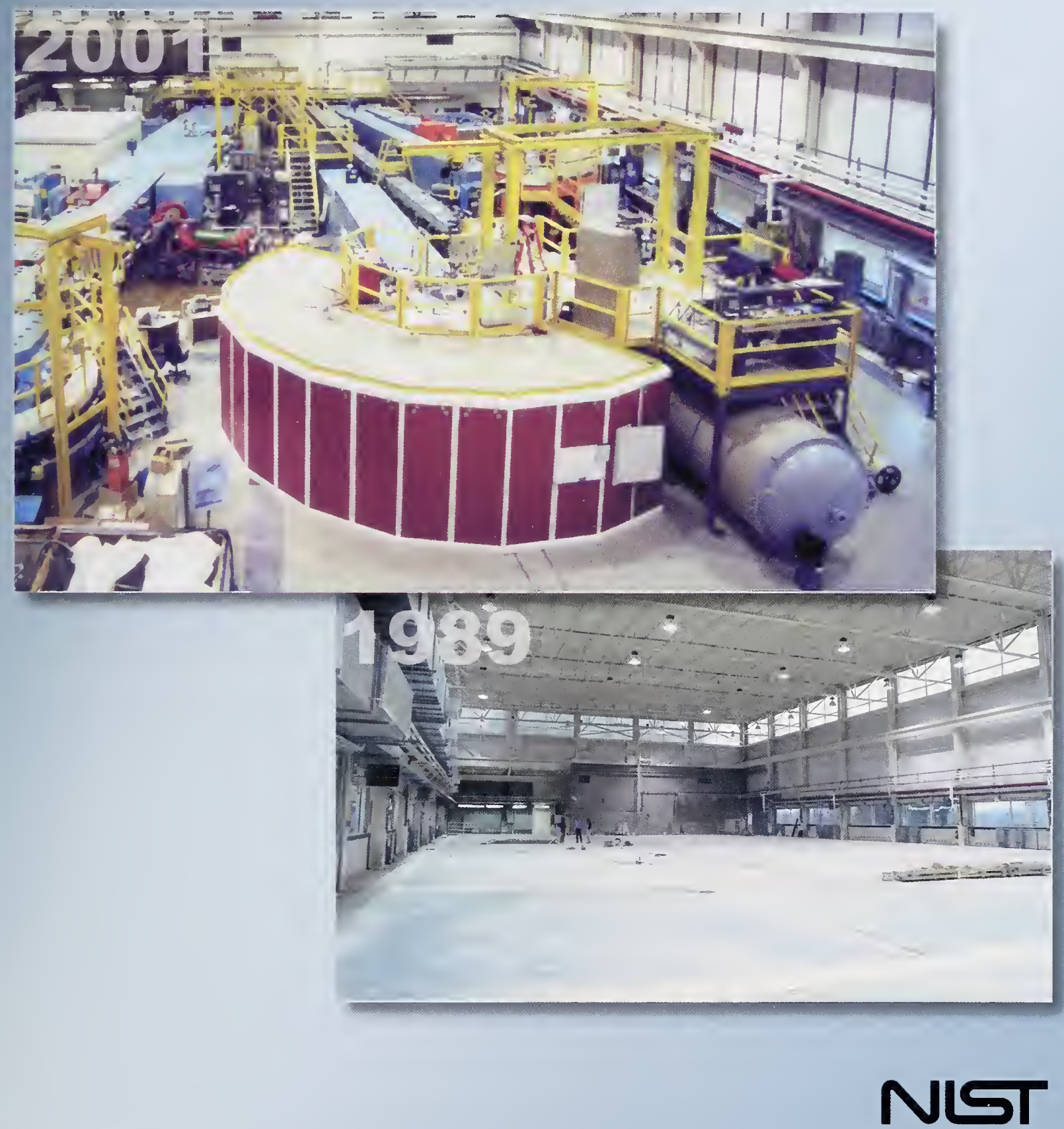

National Institute of Standards and Technology

Technology Administration 


\section{On the Cover}

The two photos dramatize the development of the NIST Center for Neutron Research over a 12-year period. The lower photo shows the Cold Neutron Guide Hall before installation of equipment. The upper picture, taken from approximately the same vantage point, is packed with 7 guide tubes serving 13 instrument stations. See pages 2-3 for a layout and brief description of the instruments. Pages 4-5 provide a selected pictorial history of the Center. (Top photo by L. A. Shuman.) 


\section{NCNR 2001}

\section{NIST}

Center for

Neutron

Research

\section{Accomplishments}

and

\section{Opportunities}

\section{NIST Special Publication 977}

J. Michael Rowe, Director

Ronald L. Cappelletti, Editor

Linda K. Clutter, Assistant Editor

March 2002

U.S. DEPARTMENT OF COMMERCE

Donald L. Evans, Secretary

Technology Administration

Phillip J. Bond, Under Secretary for Technology

National Institute of Standards and Technology

Arden L. Bement, Jr., Director

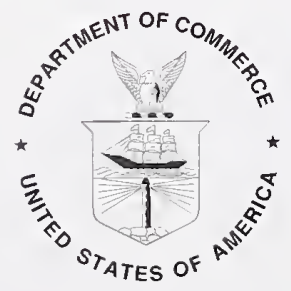


DISCLAIMER

Certain commercial entities, equipment, or materials may be identified in this document in order to describe an experimental procedure or concept adequately. Such identification is not intended to imply recommendation or endorsement by the National Institute of Standards and Technology, nor is it intended to imply that the entities, materials, or equipment are necessarily the best available for the purpose.

National Institute of Standards and Technology Special Publication 977

Natl. Inst. Stand. Technol. Spec. Publ. 977, 76 pages (March 2002)

CODEN: NSPUE2

U.S. GOVERNMENT PRINTING OFFICE - WASHINGTON: 2001

For sale by the Superintendent of Documents, U.S. Government Printing Office

Internet: bookstore.gpo.gov — Phone: (202) 512-1800 — Fax: (202) 512-2250

Mail: Stop SSOP, Washington, DC 20402-0001 
Foreword

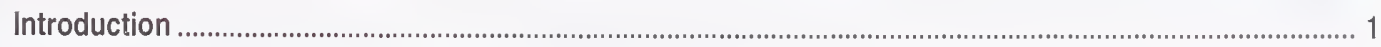

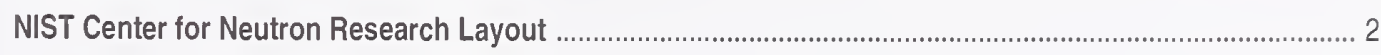

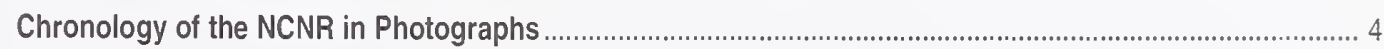

\section{Research Highlights}

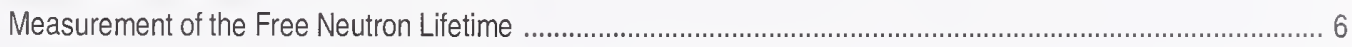

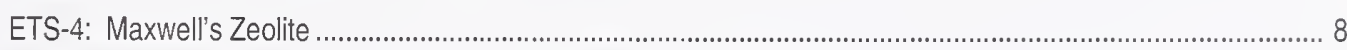

Measuring Stress Relief in Electron Beam Weld Joints of Superalloys ..................................................... 10

EXPGUI: Smoothing the Path to Powder Crystallography ............................................................................. 12

Neutron Diffraction Contributes to Improving the Fatigue Life of Bridges ...................................................... 14

Capillary Neutron Lens for Prompt Gamma Activation Micro-Analysis ...................................................... 16

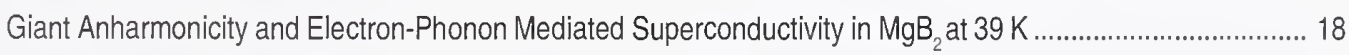

Polaron Formation and Colossal Magnetoresistance in Manganites .......................................................2 20

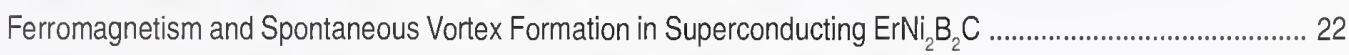

Structure of Local Spin Excitations in a Geometrically Frustrated Antiferromagnet ......................................... 24

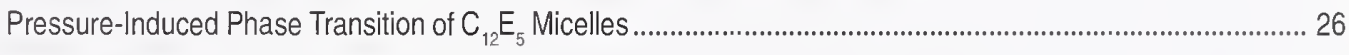

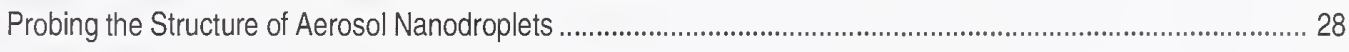

Nanoporous Ultra-Low Dielectric Constant Materials .................................................................................. 30

Neutron Reflectivity Studies of Surfactants at Electrode Surfaces ........................................................... 32

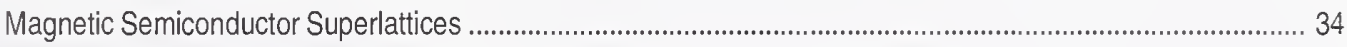

Pinpointing Chiral Structures With Front/Back Polarized Neutron Reflectometry ........................................... 36

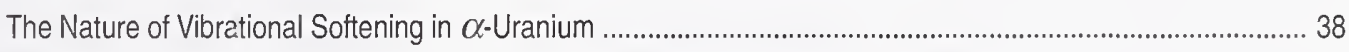

Native and Partially Unfolded Proteins: Neutron Inelastic Scattering and Simulations ..................................... 40

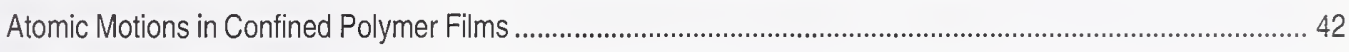

Tuning the Properties of Carbon Nanotubes by Deformation ........................................................................ 44

Serving the Scientific and Technological Communities ......................................................................... 46

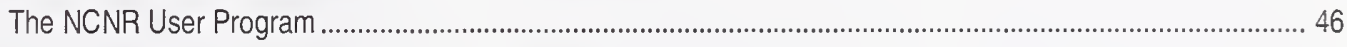

The Center for High Resolution Neutron Scattering .................................................................................. 46

Summer School on Methods and Applications of Neutron Spectroscopy ...................................................... 47

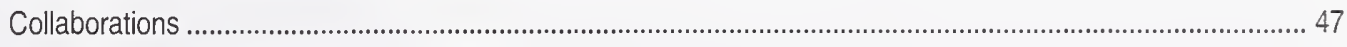

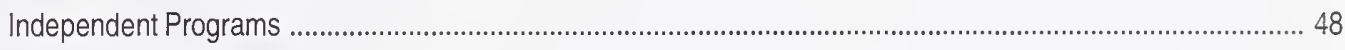

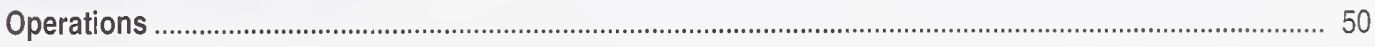

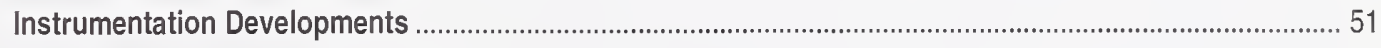

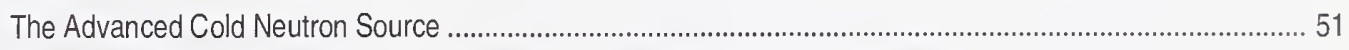

Development of an Air-Pad and Floor System for New Neutron Spectrometers ............................................. 52

Development of an Intercalated Graphite Monochromator ......................................................................... 52

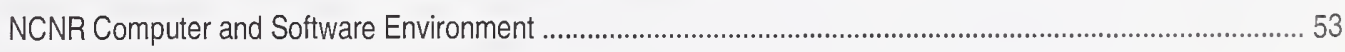

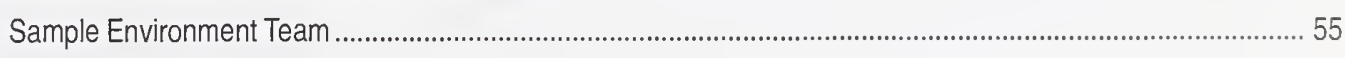

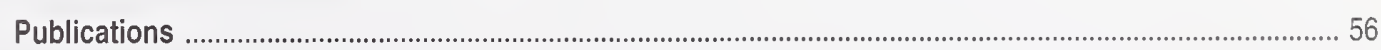

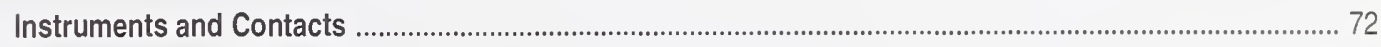


A $s$ this is being written, we have just begun a three-

month shutdown during which we will, among other changes, install a next-generation liquid hydrogen cold source, which will increase cold neutron intensities by almost a factor of two. This is an excellent example of the necessary operating philosophy for any facility - improvement and change are the only constants. During the past year, we have had many changes in reactor systems, in instrumentation, in staff, and in science, all of which are aimed at continuing the development of the NIST Center for Neutron Research as a first rate neutron facility.

A new cooling tower, which is specially designed to reduce the visible vapor plume during winter months, as well as to provide full cooling ability for the next 20 years, has been constructed. It will be connected into the reactor secondary systems during the shutdown that has just started. A new analysis of major reactor systems has been initiated using the most modern tools as part of preparing the application for a license extension through 2024. Many other improvements, ranging from instrumentation renewal to better provision for maintenance to improved electrical distribution, are under way as part of the preparation for relicensing. In spite of the shutdown that just started, the reactor has once again performed admirably, operating for 240 days during the fiscal year, meeting our scheduled operating time.

The three new inelastic scattering instruments, which were just coming on line last year, are now being fully incorporated into the user program, with encouraging user demand. The joint National Science Foundation (NSF)/NIST Center for High Resolution Neutron Scattering is being expanded to include operation of these instruments in addition to the prior SANS and triple axis instruments. The thermal neutron upgrade program, which includes two new triple axis spectrometers, an improved filter analyzer spectrometer, and other instruments, is advancing well, with characterization of monochromator crystals now complete, and contracts awarded for the manufacture of monochroma- tor drums and focusing devices. A new cold neutron triple axis spectrometer is currently entering detailed design phase as a joint construction project with Johns Hopkins University (partially funded by the NSF). In order to create more research space in the guide hall, a new building for our technical support activities is nearing completion. In response to many recommendations, we have significantly strengthened our sample environment support and our data acquisition and analysis computing effort, adding additional staff and refocusing efforts. The number of research participants continues to grow, surpassing all of our expectations. Overall, we are moving forward on many fronts to strengthen our research facility operations efforts, in order to better serve our customers.

And, as always, the scientific results are the true measure of success, and the highlights that form the largest portion of this report comprise an excellent set of examples of the exciting outputs of the facility. I hope that you enjoy reading them as much as $I$ did.

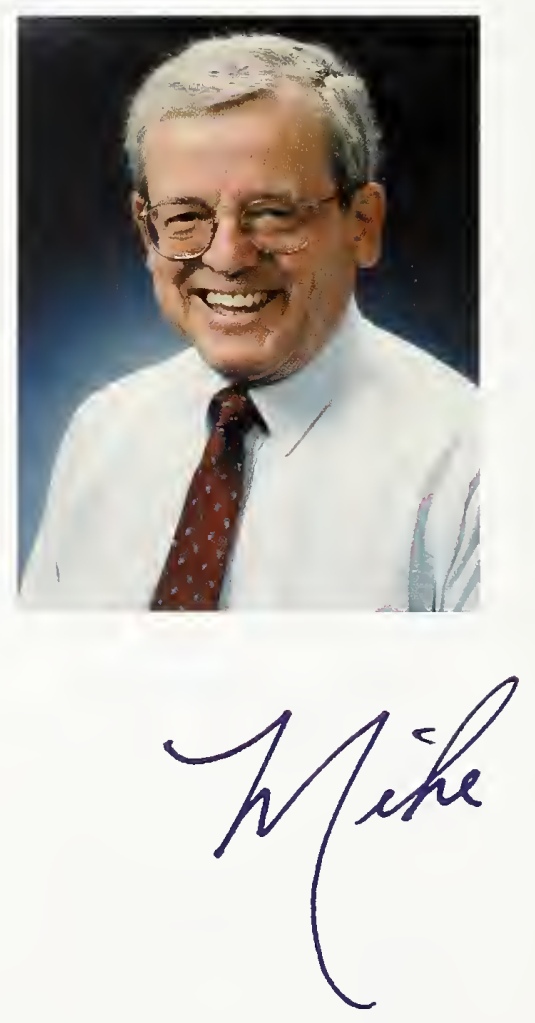




\section{Introduction to the NIST Center for \\ Neutron Research (NCNR)}

$\mathbf{M}$ odern technological society is dependent upon increasingly sophisticated use of materials with attributes dictated by their sub-microscopic structural and dynamical properties. A wide range of techniques provides knowledge of these properties. Scattering probes (for example: x-rays, light, electrons, neutrons) are arguably the most important. Of these probes, neutrons are perhaps least familiar, but they provide important advantages for many types of measurements.

Modern sources provide neutrons moving at speeds comparable to those of atoms at room temperature, thus providing the ability to probe dynamical behavior. Neutrons are also well matched to probe lengths ranging from the distances between atoms to the size of biological or polymer macromolecules. Neutrons are sensitive to the magnetic properties of atoms and molecules, allowing study of the underlying magnetic properties of materials. They also scatter differently from normal hydrogen atoms than they do from heavy hydrogen (deuterium), allowing selective study of individual regions of molecular systems. Finally, neutrons interact weakly with materials, providing the opportunity to study samples in different environments more easily (at high pressures, in shear, in reaction vessels, etc.), and making them a non-destructive probe. These favorable properties are offset by the weakness of the best neutron sources compared to x-ray or electron sources, and by the large facilities required to produce neutrons. As a result, major neutron sources are operated as national user facilities to which researchers come from all over the United States and abroad to perform small-scale science using the special measurement capabilities provided.

In addition to scattering, neutrons can be captured by nuclei to probe the atomic composition of materials. The subsequent characteristic radioactive decays provide "fingerprints" for many atomic nuclei, allowing studies of environmental samples for pollutants (e.g., heavy metals), characterization of Standard Reference Materials, and other measurements. There are important areas in physics that can be explored by measuring fundamental neutron behavior. Examples include the lifetime of the free neutron, important for the theory of astrophysics; the neutron beta decay process, that provides a stringent test of nuclear theory; and the effects of various external influences such as gravity or magnetic fields on neutrons.

The NCNR's 20-MW NIST Research Reactor provides a national user community with facilities, including the nation's only internationally competitive cold neutron facility, for all of the above types of measurements. There are about 35 stations in the reactor and its associated beams that can provide neutrons for experiments. At the present time 28 of these are in active use, of which 6 provide high neutron flux positions in the reactor for irradiation, and 22 are beam facilities. The following pages show a schematic layout of the facility. More complete descriptions of instruments can be found at http://www.ncnr.nist.gov.

These facilities are operated both to serve NIST mission needs and as a national facility, with many different modes of access. Some instrumentation was built years ago, and is not suited to general user access; however, time is available for collaborative research. NIST has recently built new instrumentation, and reserves $1 / 3$ of available time for mission needs with the balance available to general users. In other cases, instrumentation was built and is operated by Participating Research Teams (PRT); PRT members have access to $75 \%$ of available time, with the balance available to general users. Additionally, NIST and the National Science Foundation operate the Center for High Resolution Neutron Scattering at the NCNR, with one thermal and five cold neutron instruments. For these facilities, most time is available for general users. While most access is for research, with results that are freely available to the general public, proprietary research can be performed under full cost recovery. Each year, about 1600 researchers (persons who participated in experiments at the facility, but did not necessarily come here) from all areas of the country, from industry, academia, and government use the facility for measurements not otherwise possible. The research covers a broad spectrum of disciplines, including chemistry, physics, biology, materials science, and engineering. 

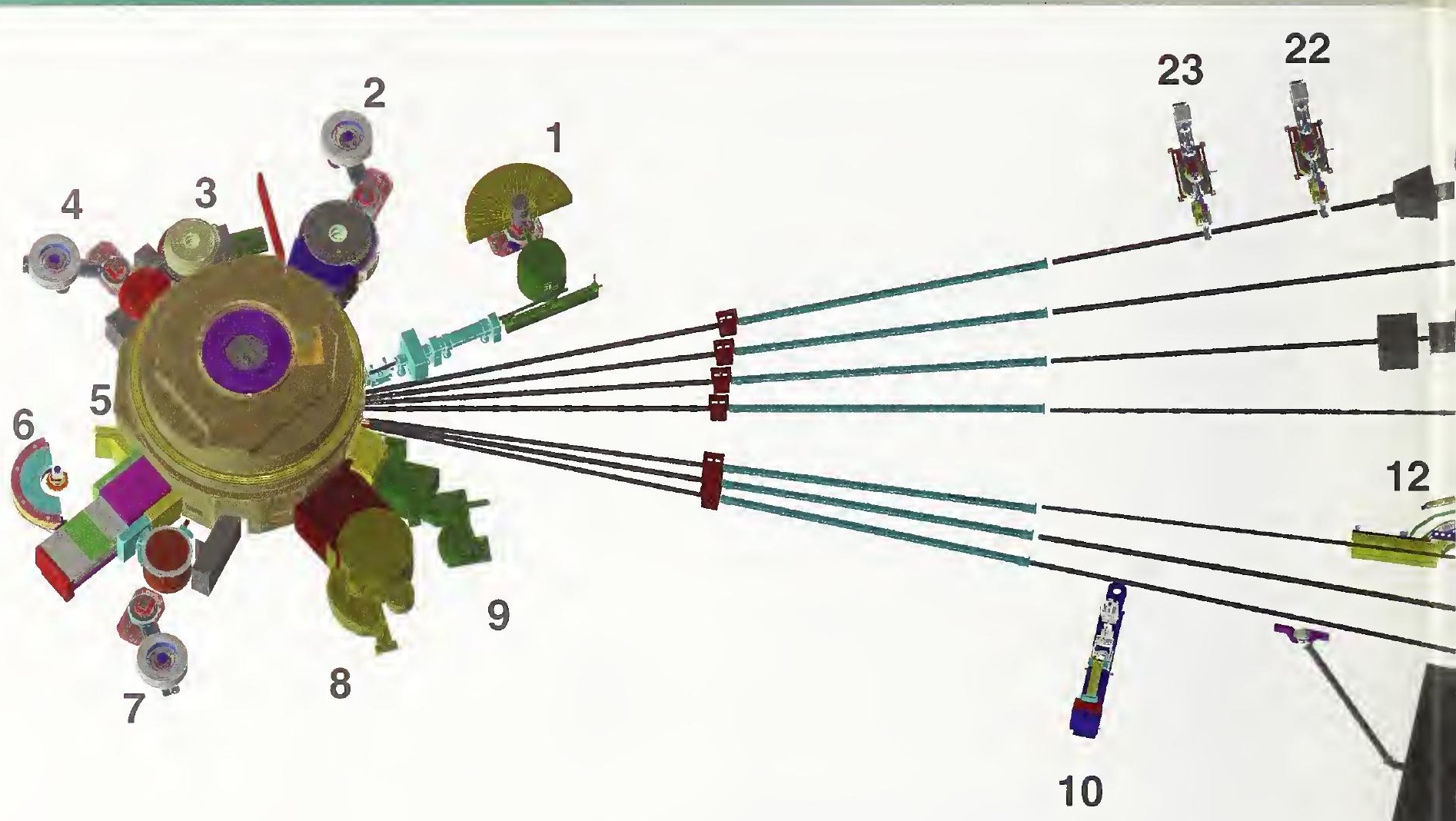

1 A Cold Neutron Depth

Profiling instrument (not shown) for quantitative profiling of subsurface impurities currently at this site will be moved to another position. Shown is a proposed Triple Axis Cold Neutron Crystal Spectrometer with double focusing monochromator and multiple crystal analyzer/ detectors that can be flexibly configured for several energies simultaneously or for high throughput at one energy.

2 BT-7 Triple Axis Spectrometer with fixed incident energy for measurements of excitations and structure.
3 BT-8 Residual Stress Diffractometer optimized for depth profiling of residual stress in large components.

4 BT-9 Triple Axis Crystal Spectrometer for measurements of excitations and structure.

5 Thermal Column A very wellthermalized beam of neutrons used for radiography, tomography, dosimetry and other experiments.

6 BT-1 Powder Diffractometer with 32 detectors; incident wavelengths of $0.208 \mathrm{~nm}$, $0.154 \mathrm{~nm}$, and $0.159 \mathrm{~nm}$, with highest resolution of $\delta \mathrm{d} / \mathrm{d}=$ $8 \times 10^{-4}$.
7 BT-2 Triple Axis Crystal Spectrometer with polarized beam capability for measurement of magnetic dynamics and structure.

8 BT-4 Filter Analyzer Neutron Spectrometer with cooled Be/ Graphite filter analyzer for chemical spectroscopy.

\section{BT-5 Perfect Crystal} Diffractometer SANS small angle neutron scattering instrument for microstructure on the $10^{4} \mathrm{~nm}$ length scale, sponsored by the National Science Foundation and NIST, part of the Center for High Resolution Neutron Scattering (CHRNS).
10 NG-7 Horizontal Sample Reflectometer allows reflectivity measurements of free surfaces, liquid vapor interfaces, as well as polymer coatings.

11 Neutron Interferometry and Optics Station with perfect silicon interferometer; vibration isolation system provides exceptional phase stability and fringe visibility.

12 Spin Polarized Triple Axis Spectrometer (SPINS) using cold neutrons with position sensitive detector capability for high resolution studies - part of CHRNS. 


\section{Chronology of the NCNR in Photographs}

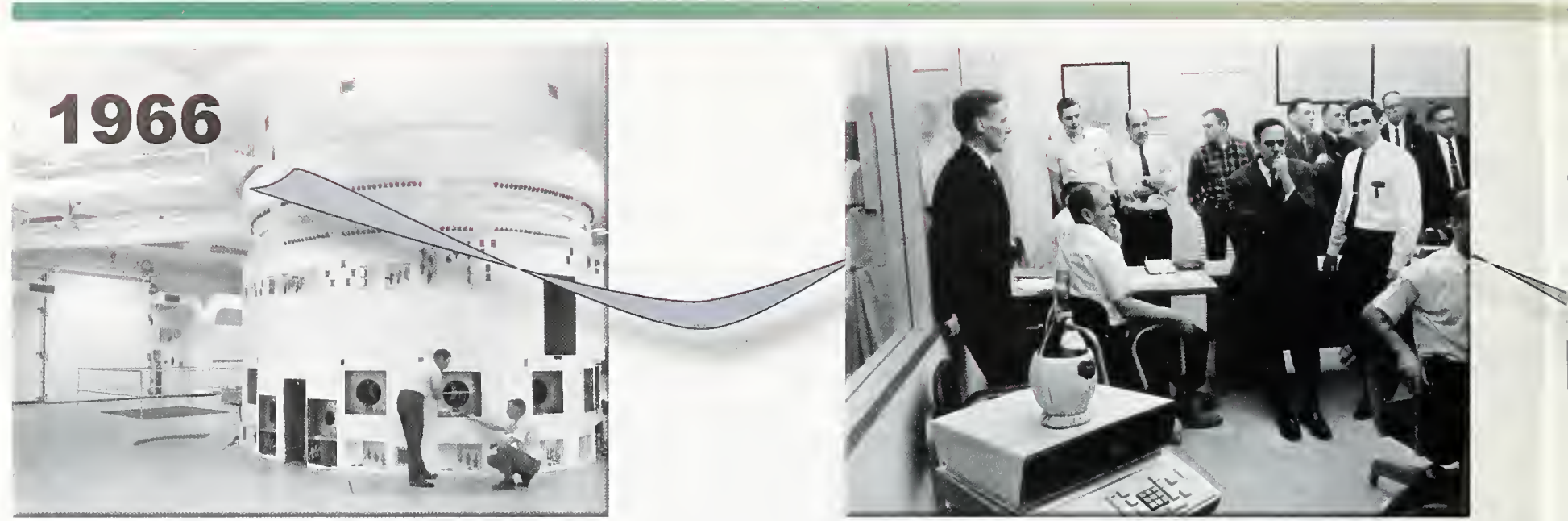

Fred Shorten and Chuck Ware at beam tube opening (BT-4) during construction in 1966.

Awaiting criticality, December 7, 1967. Chianti not yet uncorked.

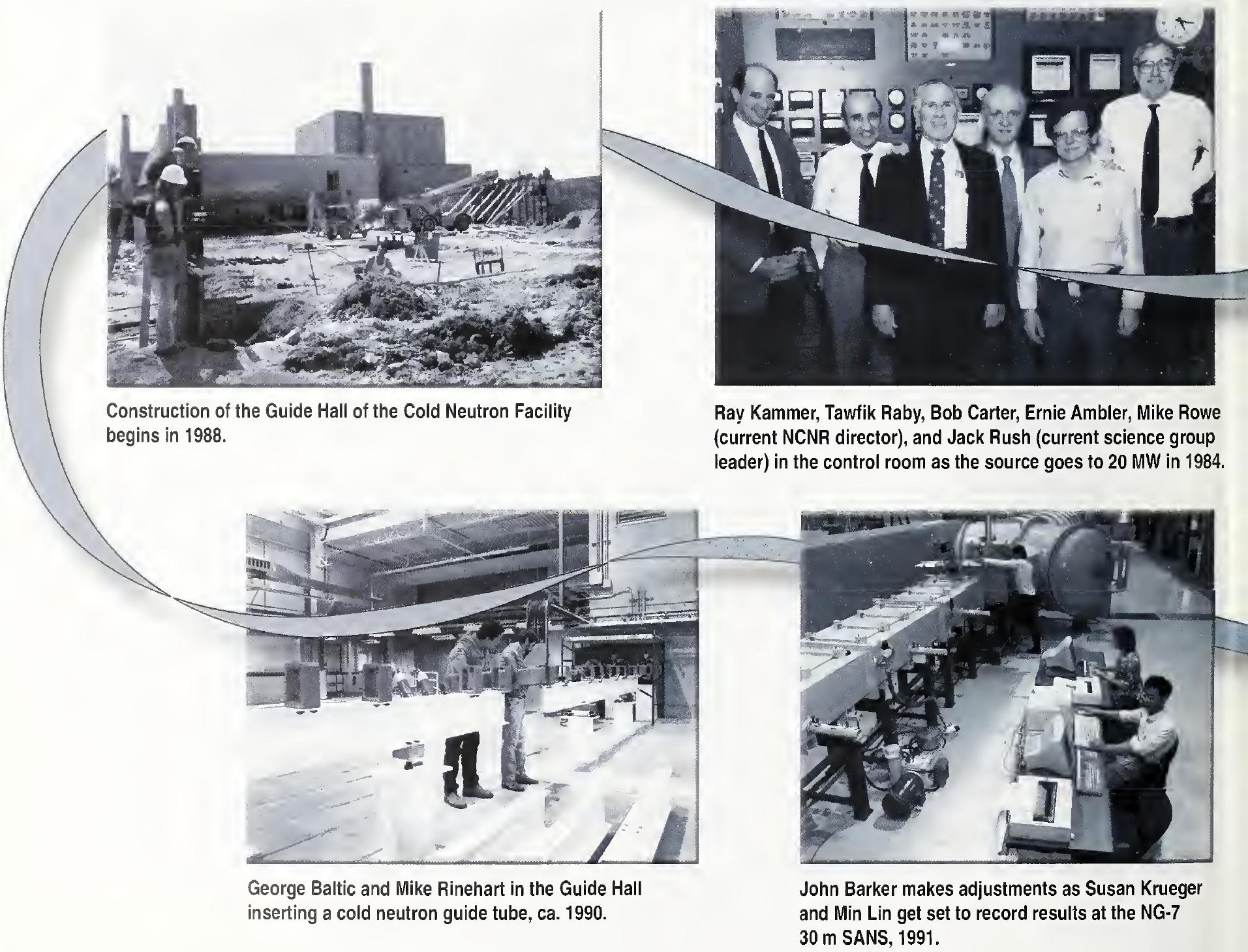




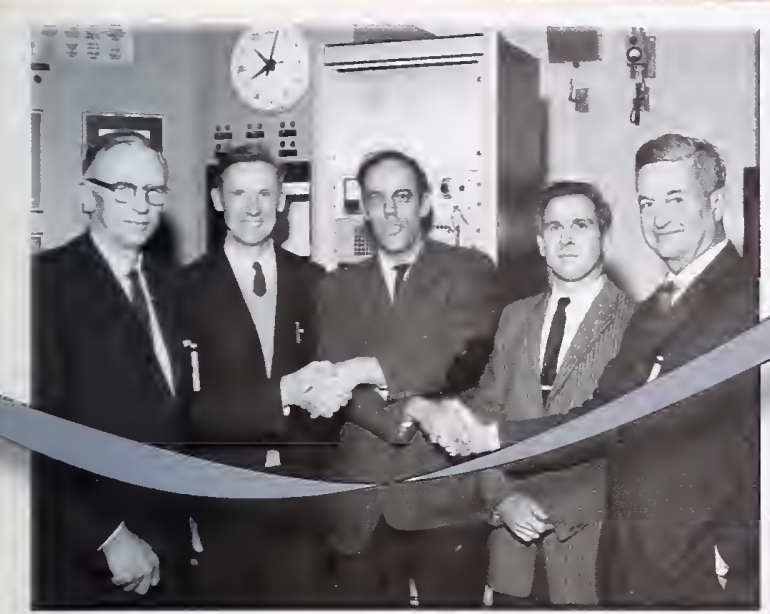

Anticipating success, Allen Astin, Harry Landon, Carl Muehlhause, Robert Carter, and IrI Schoonover.

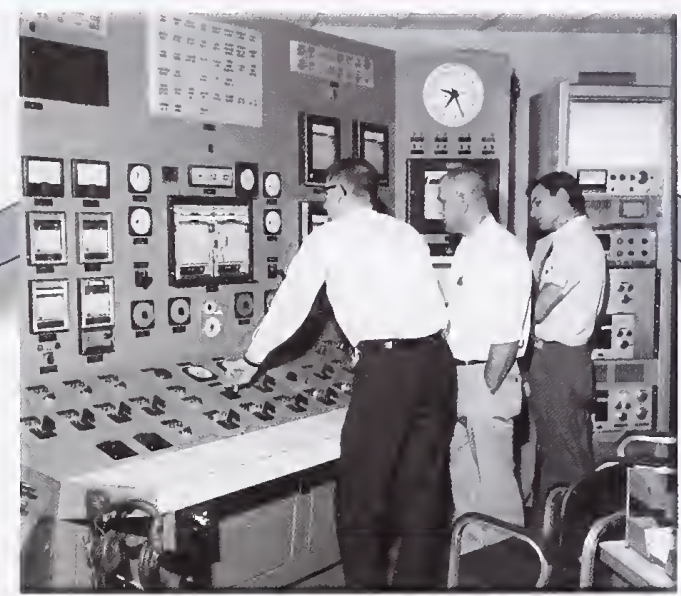

Larry Smith, Art Chatman and Bill Crebs, at the controls during criticality test.

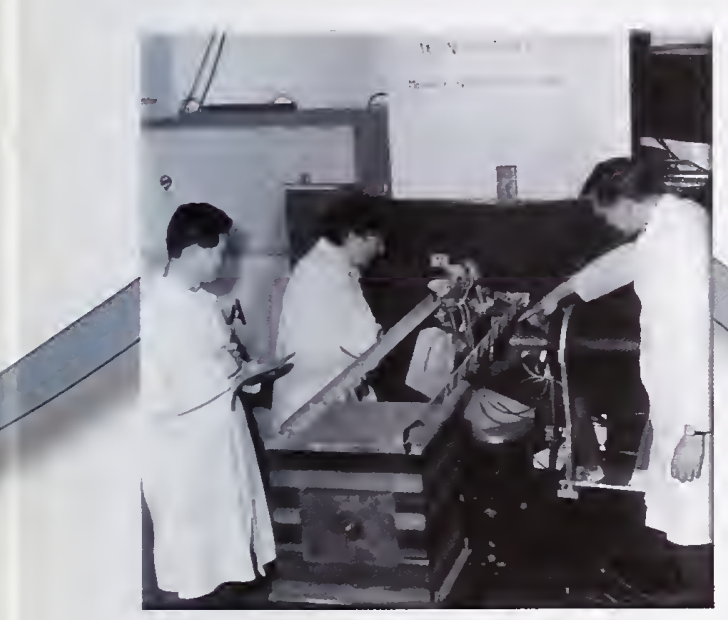

Chang-Sun Choi, Sam Treviño and Henry Prask at a 2-axis diffractometer sited at BT-6, 1973.

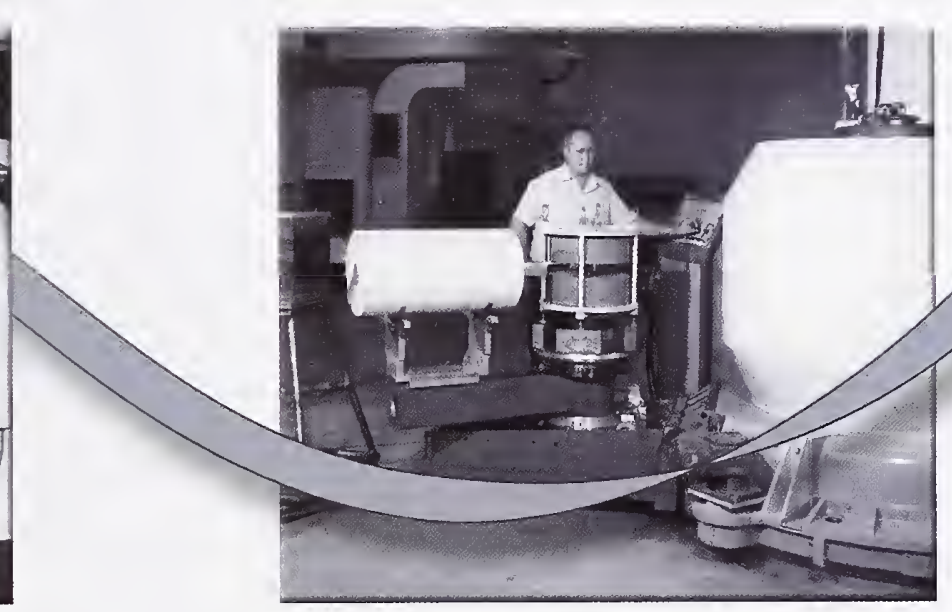

Al Cinquepalma at an early version of the diffractometer at BT-1, 1971.

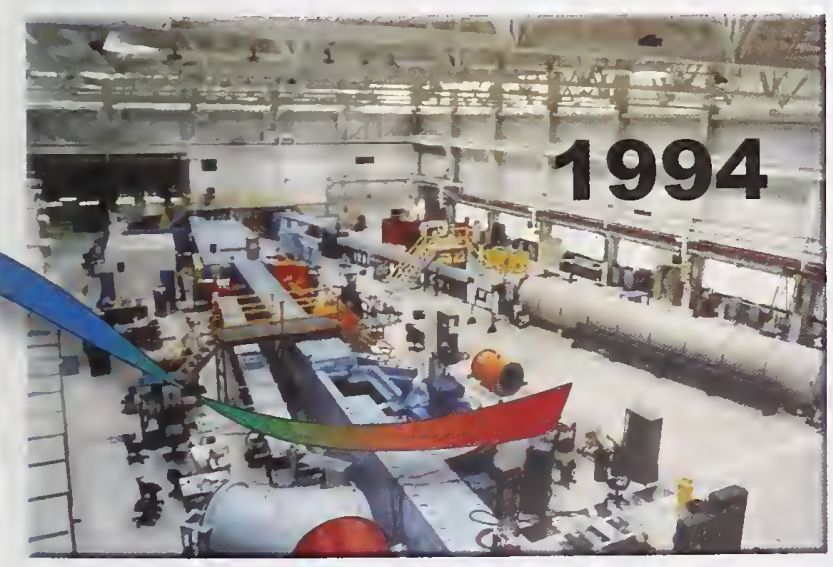

The Guide Hall begins

to fill up, 1994:

NG-7 $30 \mathrm{~m}$ SANS at left, NG-6 Fermi-chopper time-of-flight spectrometer, center, $8 \mathrm{~m}$ SANS (orange) behind it, and NG-3 $30 \mathrm{~m}$ SANS at right. Also visible is the chamber housing the neutron interferometer at far left, back. See front cover for an early and recent view. 


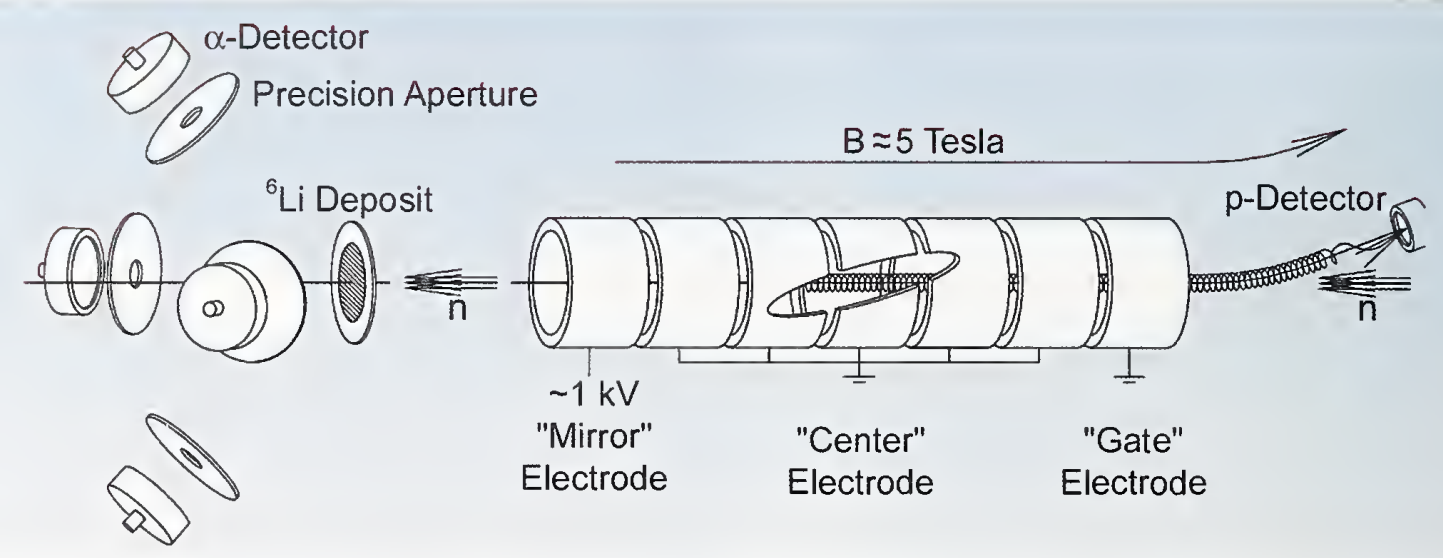

FIGURE 1. Schematic drawing of the NIST Penning trap neutron lifetime experiment.

$\mathbf{F}$ ree neutrons are unstable and decay with a lifetime of about 15 minutes into a proton, an electron, and an antineutrino. Accurate determination of this natural lifetime is important for tests of the Standard Model of Fundamental Particles and Interactions, as well for understanding certain aspects of cosmology and astrophysics. The neutron lifetime influences the predictions of the theory of Big Bang nucleosynthesis for the primordial helium abundance in the universe and the number of different types of light neutrinos. Since the dominant uncertainty for the primordial helium abundance comes from the accuracy of the neutron decay rate [1], improved neutron decay rate measurements are needed for sharpening this prediction.

The measurement of the lifetime of free neutrons in a cold neutron beam was one of the first experiments operating in the NCNR Guide Hall. The experiment occupied the NG-6 Physics Station alternatively with other fundamental physics experiments through December 2000. A statistical precision of one part per thousand was achieved in the final result. An overall standard uncertainty of about four parts per thousand has been obtained, and that uncertainty may be eventually reduced by as much as a factor of two by ongoing developments in characterization of the neutron counting for this experiment.

Figure 1 shows a schematic diagram of the apparatus. An axial 5 Tesla magnetic field provides radial containment of the decay protons, while axial containment is achieved by the positive electrostatic charge on two end electrodes, called the mirror and the gate. This combination of mag- netic and electrostatic fields is called a "Penning Trap." The neutron detector consists of a well-characterized isotopic target of ${ }^{6} \mathrm{Li}$, viewed by a set of four charged particle detectors. The charged particles from neutron reactions in the isotopic targets are counted with an accuracy of better than one part per thousand. The reaction cross sections for these standard cross sections are known to about $0.25 \%$.

When a neutron in the beam decays inside the trapping region, the recoil proton is trapped. Periodically, the gate electrode is lowered to ground potential to allow the accumulated trapped proton(s) to exit the trap and be counted by the proton detector. A bend in the magnetic field makes it possible to locate the proton detector outside of the incoming neutron beam. The trapped protons have a maximum energy of less than $800 \mathrm{eV}$ and are undetectable at those low energies, but by holding the proton detector at a negative high voltage of the order of $-30 \mathrm{kV}$, the protons may be detected with a signal well above noise levels. Counting for only a short time (less than $100 \mu$ s) after the trap is opened in each counting/trapping cycle further enhances the signalto-noise ratio. Typical trapping times are of the order of $10 \mathrm{~ms}$, and typical decay event rates are of the order of a few protons per second.

Determination of the lifetime requires either an accurately known trap length or a variable trap length with accurately known differences in length. The latter is more easily realized physically and is achieved in this apparatus by the precision machining of the segmented trap structure. Varying the trap length by applying the mirror voltage to 
M. S. Dewey, D. M. Gilliam, J. S. Nico, and F. E. Wietfeldt lonizing Radiation Division National Institute of Standard's and Technology Gaithersburg, MD 20899-8461
W. M. Snow

Indiana University Cyclotron Facility

Indiana University

Bloomington, IN 47405-7105
G. L. Greene

Los Alamos National Laboratory

Los Alamos, NM 87545 different segments does not significantly change the hard-todefine end effects. Figure 2 shows a plot of the measured proton rate (after normalization to the neutron rate) as a function of trap length; the slope of this line is inversely proportional to the neutron lifetime.

Since the time when some of the present staff of this experiment participated in a closely related experiment [2] at the Institut Laue-Langevin, a large number of changes have been made to improve the accuracy. Perhaps the most important factor was simply having much more beam time

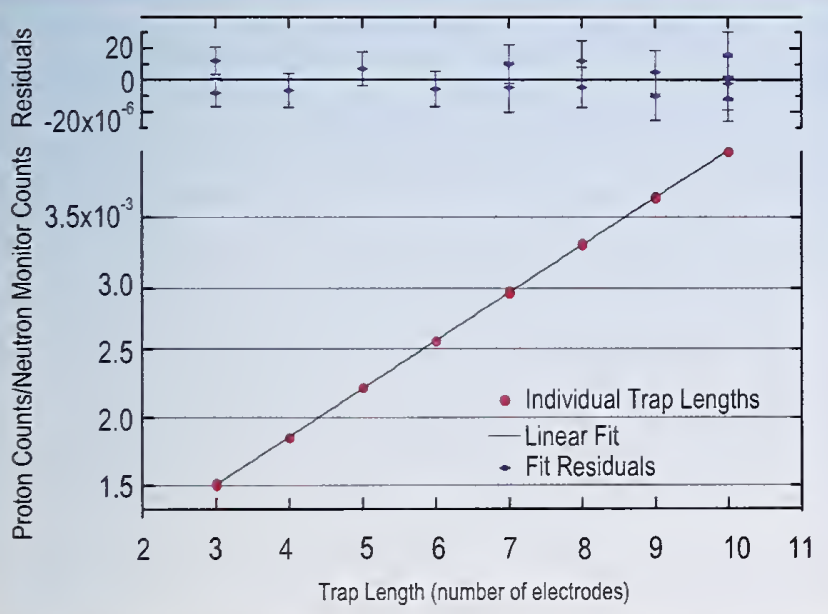

FIGURE 2. A plot of the proton count rate (normalized to the neutron monitor count rate) vs. trap length. The slope of the line is inversely proportional to the measured neutron lifetime.

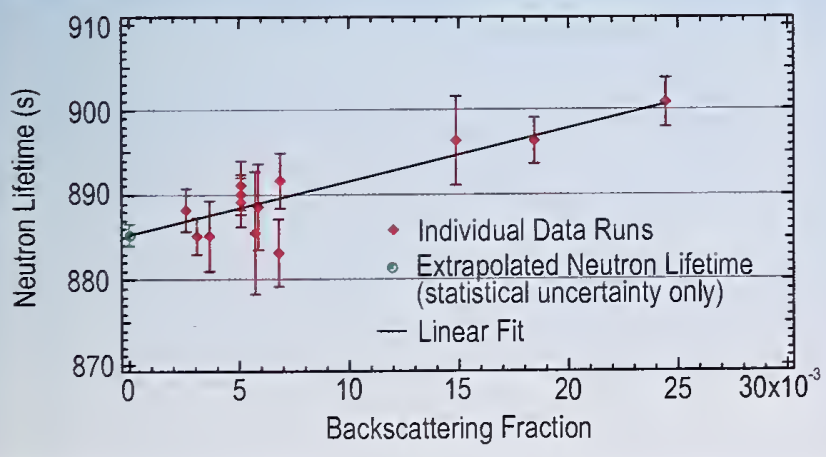

FIGURE 3. A linear fit of the measured neutron lifetime at varying values of the detector backscattering fraction. The extrapolation to zero backscattering gives the free neutron lifetime. for evaluation of significant systematic effects and accumulation of better statistical precision. Other factors include Monte Carlo-computed corrections due to magnetic field inhomogeneities, improved beam profiles and proton alignment, better trap voltage stability and monitoring, improved definition of the areal mass density of the isotopic targets, use of a new trap designed to minimize instabilities, and improved analysis methods.

Figure 3 shows the extrapolation of the measured lifetime as a function of the backscattering fraction for protons from the detector surface to zero backscattering. The preliminary lifetime value obtained in this experiment [3] is:

$$
885.3 \mathrm{~s} \pm 4.0 \mathrm{~s} \text {. }
$$

Continuing efforts to measure the neutron count rate are underway by both calorimetric and coincidence techniques, which should reduce the final uncertainty by half. They will not only improve on the results of this experiment but will also provide a more accurate and direct calibration of the NIST Standard Neutron Source.

Also underway at NG-6 is a new experiment to measure the neutron lifetime by an independent method involving magnetic trapping of ultracold neutrons. This experiment is being carried out in collaboration with the Physics Department of Harvard University.

\section{References}

[1] S. Burles, K. M. Nollett, J. W. Truran, and M. S. Turner, Phys. Rev. Lett. 82 4176, (1999).

[2] J. Byrne et al., Phys. Rev. Lett. 65, 289 (1990); J. Byrne et al., Europhys. Lett. 33, 187 (1996).

[3] M. S. Dewey et al., manuscript in preparation. 
cottish physicist James Clerk Maxwell (1831-1879)

discussed a mythical device that could separate molecules by temperature, a concept now known as Maxwell's demon. Maxwell used this idea to explore how sorting molecules by size would violate the second law of thermodynamics. If one did have a Maxwell's demon, a more useful task for it would likely be to separate molecules by size. This would allow mixtures to be separated into pure substances, and would facilitate removal of dangerous contaminants from valuable materials. In some cases, molecular separations can be performed using zeolites and similar materials. Zeolites are composed of linked tetrahedral $\mathrm{SiO}_{4}$ and $\mathrm{AlO}_{4}$ species, arranged in rings to create a framework of molecule-sized cavities. Related zeolitic materials may use other tetrahedral atoms to build the framework. Molecules can access these cavities or pores, under two conditions: if they can pass through the appropriate rings in the framework and these rings are not blocked by other species in the material, such as cations. If the ring size in a material matches the needs for a particular chemical separation, the material may be appropriate. Chemical modifications, such as ion exchange or changing the framework composition can sometimes influence the pore size. So, occasionally it is possible to modify a zeolite to a particular separation. However, this does not allow what Maxwell might envision, where the pore size can be adjusted over a wide range to suit the desired process, in effect a Maxwell's demon for size separations. This exact capability has now been demonstrated in material ETS-4 [1]. ETS-4 can be processed in such a way so that the pore sizes can be tuned for particular separations.

ETS-4 is a titanosilicate material invented by Engelhard in the 1980s [2]. ETS-4 differs from most zeolitic materials in a number of ways. Chemically, it is an oxide of $\mathrm{Ti}$ and $\mathrm{Si}$, while the majority of zeolitic materials are oxides of $\mathrm{Al}$ and $\mathrm{Si}$. Structurally, the material is composed of both tetrahedral $\mathrm{TiO}_{4}$ and octahedral $\mathrm{TiO}_{6}$ units, as well as $\mathrm{SiO}_{4}$ units. Finally, most zeolitic materials hydrate and dehydrate reversibly or are destroyed during dehydration. ETS-4, at least in some ion-exchanged forms, can be induced to dehydrate irreversibly and without destruction of the pores. Dehydration reduces the average pore opening size. Thus it

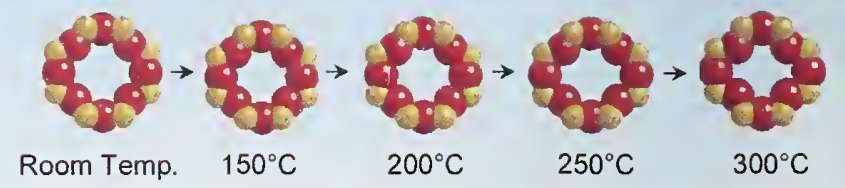

FIGURE 1. Size of the eight-ring pore opening in ETS-4 as a function of dehydration temperature.

is possible to tune the zeolite to accept molecules of a particular size by changing the level of dehydration. The temperature to which the material is exposed in turn dictates the amount of dehydration. In 1999, the Advanced Technology Program funded Engelhard to exploit this effect, which Engelhard calls the Molecular Gate ${ }^{\circledR}$, to develop methods for separating oxygen from air, a process with useful applications for prevention of pollution as well as medical and other industrial applications.

The mechanism behind the Molecular Gate ${ }^{\circledR}$ effect was demonstrated crystallographically using data from the BT-1 neutron powder diffractometer. Pores in ETS-4 must be accessed via a ring of eight $\mathrm{Si}$ and $\mathrm{Ti}$ atoms, each linked by oxygen atoms. This ring is commonly called an eight-ring, despite the fact that it is actually composed of sixteen atoms. In Fig. 1, the size of this eight-ring is shown as a function of dehydration temperature. The actual size of molecules that can be admitted via this opening are dictated by the van der Waals radii of the $\mathrm{O}$ atoms in the eight-ring and the van der Waals dimensions of the molecule to be admitted. Since accurate determination of oxygen atom siting is needed, and since ETS-4 does not form single crystals, neutron powder

TABLE 1. Eight-ring opening as a function of dehydration temperature. Distances are the pore-openings separating van der Waals radii between opposite $05\left(D_{1}\right), 01\left(D_{2}\right)$ and $02\left(D_{3}\right)$ atom pairs (see FIGURE 2).

\begin{tabular}{c|c|c|c|c|c}
$\begin{array}{c}\text { van der } \\
\text { Waals } \\
\text { opening } \\
(\AA)\end{array}$ & \multicolumn{5}{|c}{ Dehydration Temperature, ${ }^{\circ} \mathrm{C}$} \\
\cline { 2 - 6 } & $\mathrm{RT}$ & 150 & 200 & 250 & 300 \\
\hline $\mathrm{D}_{1}$ & 4.27 & 3.97 & 3.95 & 3.94 & 3.90 \\
\hline $\mathrm{D}_{2}$ & 4.43 & 4.02 & 4.09 & 4.57 & 4.57 \\
\hline $\mathrm{D}_{3}$ & 3.61 & 3.28 & 3.29 & 3.27 & 2.77
\end{tabular}


S. M. Kuznicki, V. A. Bell and R. M. Jacubinas

Strategic Technology Group Engelhard Corporation Iselin, NJ 08830
S. Nair, H. W. Hillhouse,

C. M. Braunbarth and M. Tsapatsis Department of Chemical Engineering, University of Massachusetts

Amherst, MA 01003

\section{B. H. Toby}

NIST Center for Neutron Research

National Institute of Standards and Technology Gaithersburg, MD 20899-8562

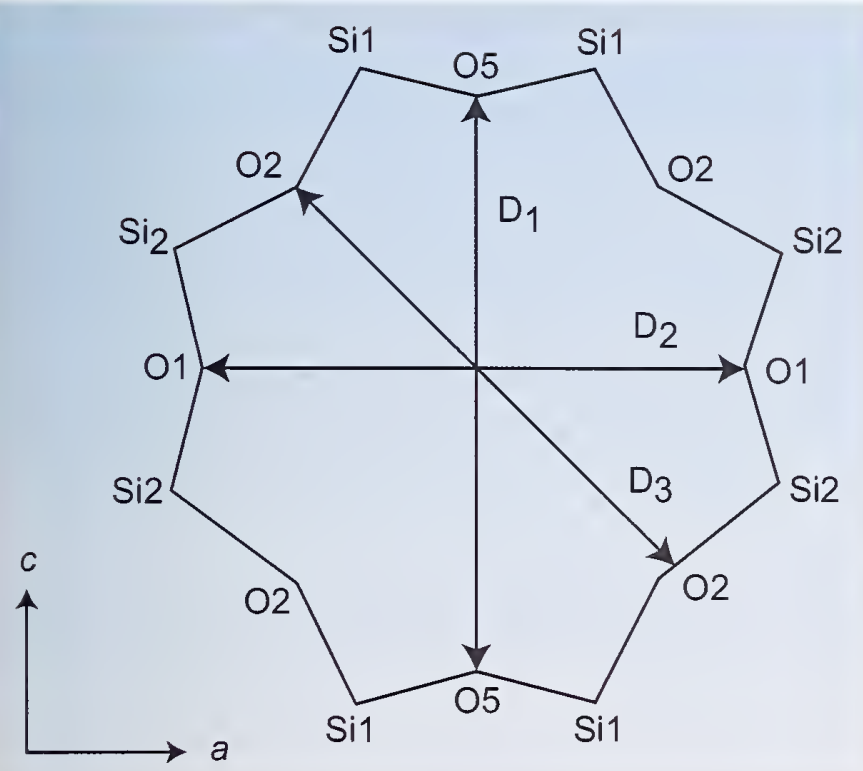

FIGURE 2. Atom and pore-opening distance labeling scheme in the eight-ring opening of ETS-4.

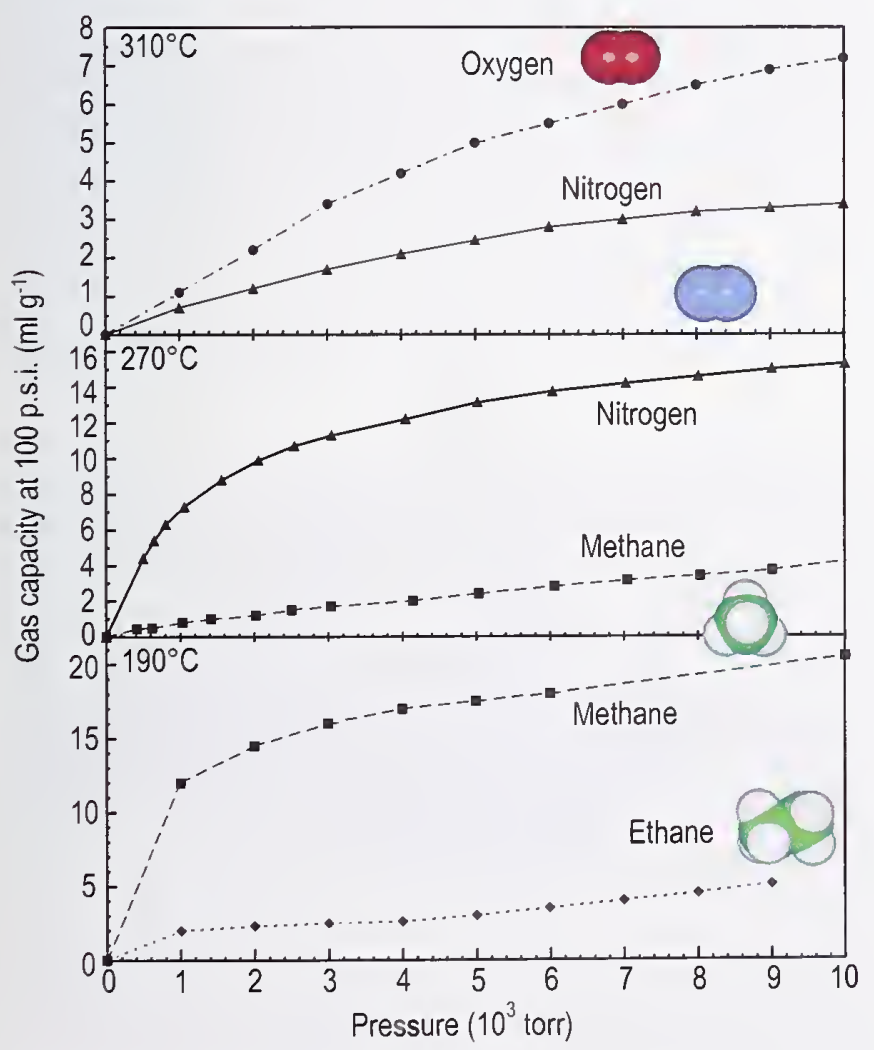

FIGURE 3. Adsorption of selected molecules as a function of dehydration temperature. diffraction measurements are the only way to obtain accurate measurements of the ring openings as a function of dehydration temperature. Measurements of the eight-ring opening from Rietveld refinements are shown in Table 1, relative to the distances diagrammed in Fig. 2.

The proof that dehydration indeed does change the pore access is demonstrated by adsorption isotherms, shown in Fig. 3. The separation shown in the top of this figure, between $\mathrm{O}_{2}$ and $\mathrm{N}_{2}$, shows how precisely the pore sizes can be adjusted. The difference in van der Waals radius between oxygen and nitrogen is only $0.1 \AA$.

\section{References}

[1] S. M. Kuznicki, V. A. Bell, S. Nair, H. W. Hillhouse, R. M. Jacubinas, C. M. Braunbarth, B. H. Toby, and M. Tsapatsis, Nature 412, 720 (2001).

[2] S. M. Kuznicki, US Patent 4938939 (1990). 


\section{Measuring Stress Relief in Electron Beam Weld Joints of Superalloys}

a ingle-crystal turbine blades are the pinnacle of a - decade-long development and refinement of nickelbased superalloys. By eliminating creep along grain boundaries as the main failure mechanism of polycrystals at high temperatures, the use of single crystal turbine blades has allowed a rise in temperature in the "hot zone" by more than $100 \mathrm{~K}$, thus substantially increasing thermodynamic efficiency of jet engines. The complete turbine blade, including the mounting base and the cooling channels, is grown from the melt in a single process and represents a considerable investment. In order to protect this investment, electron beam welding (see Fig. 1) is used to repair minor structural damage of failed components.

The weld process creates very high residual stresses that make the extended weld region prone to cracking. Cracks are sometimes found parallel to the weld especially in the $z$-direction, so that an additional heat treatment must be applied for stress relief. The standard heat treatment is $2 \mathrm{~h}$ at $1423 \mathrm{~K}$ for homogenizing (solution) and a subsequent aging treatment. In this investigation neutron diffraction is used to compare the stresses before and after heat treatment in the single crystal side of the weld joint.

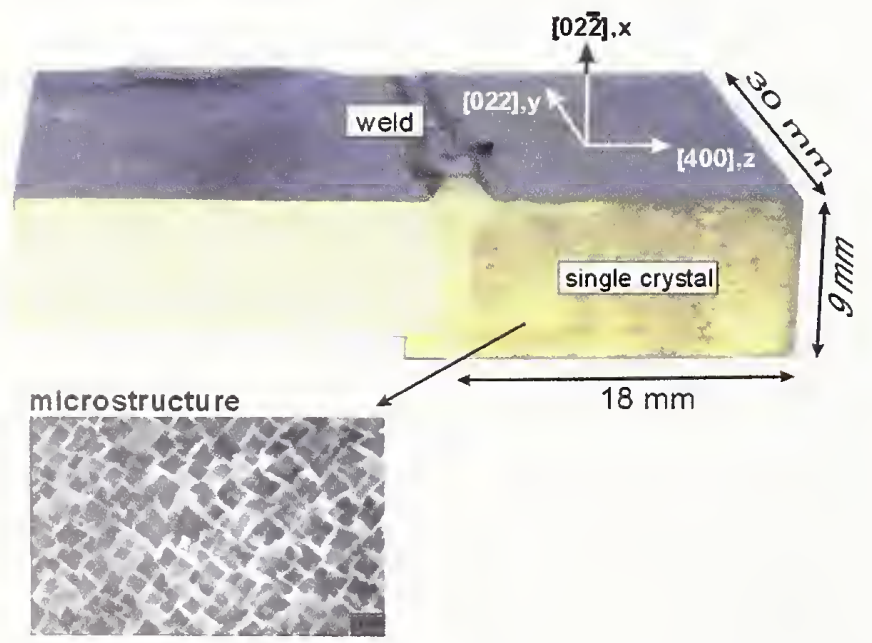

FIGURE 1. Test specimen of a weld joining a Rene N5 single crystal and a polycrystal. The three different regions, polycrystal, weld, and single crystal, appear in different colors after etching which indicates different chemical compositions. The microstructure shows typical features: $60 \%$ cuboidal $\gamma$ 'precipitates and unimodal size distribution.
Usually a polycrystalline alloy of comparable composition is chosen as a replacement material. The typical grain size of the polycrystal is approximately one millimeter, which is about the size of the incident neutron beam. Thus, stress measurements are not feasible for this side of the weld. Compared to a measurement on a fine-grained polycrystal, the measurement procedure is rather difficult because all small angle grain boundaries (Fig. 2) have to be brought into reflection and averaged over the rocking angle $\omega$ in the data reduction.

Additionally, both the welding and the heat treatment produce position-dependent chemical gradients that affect the unstressed lattice parameters. Their measurement would have required cutting the specimen into little pieces for complete stress relief. We chose an alternative approach of applying mechanical boundary conditions at the surface. The results obtained from this procedure are shown in Fig. 3.

The effect of the heat treatment is dramatic. Both the strong tensile stresses in the heat-affected zone $(5 \mathrm{~mm}$ from the weld center) and the strong gradients at the interface between weld and heat affected zones are removed. Stress relief in the $z$-direction is especially important because it removes the driving force for crack opening parallel to the weld in the heat-affected zone.

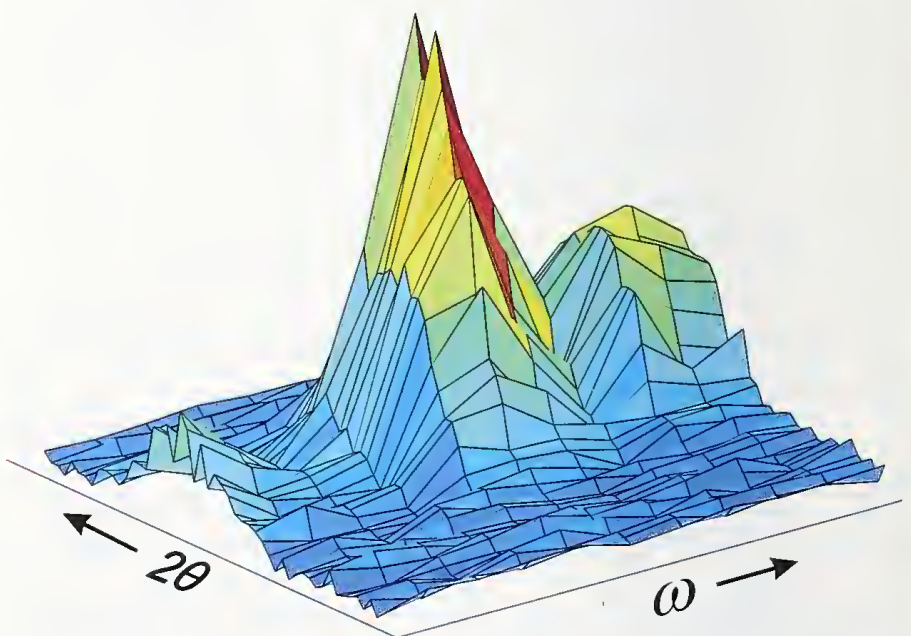

FIGURE 2. $Q_{x}$-intensity map for the as-welded sample (reciprocal space) plotted vs. $2 \theta$ and the rocking angle $\omega$. Small angle grain boundaries as indicated by the presence of several maxima are characteristic for superalloy single crystals. The existence of two maxima in $2 \theta$ (equivalent to two $d$-spacings) indicates the $\gamma$-matrix and the $\gamma^{\prime}$-precipitates whose lattice parameters are close to each other. 
Remarkable features in Fig. 4 are the strong compressive stresses after heat treatment in the middle ( $4 \mathrm{~mm}$ depth) both for the $z$-direction and the $y$-direction. There is evidence that solidification partitioning can lead to a different $\gamma / \gamma^{\prime}$-microstructure in the weld zone, thus affecting the mean lattice parameter measured by diffraction. The immediate results are apparent stresses caused by changes in the unstressed $d$-spacing. This effect can be removed with some confidence only for the measurements close to the surface ( = $1 \mathrm{~mm}$ depth) by using mechanical equilibrium condi-

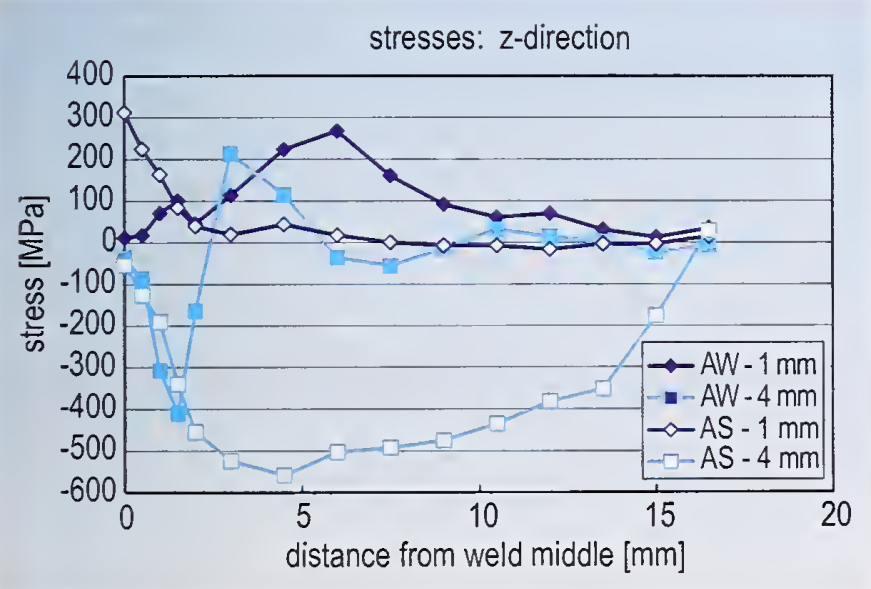

FIGURE 3. Residual stresses $\sigma_{z z}$ before and after the heat treatment for two different depths from the upper surface. AW stands for "As Welded"; AS stands for "Heat Treated." The uncertainties are of the order of the size of the data points.

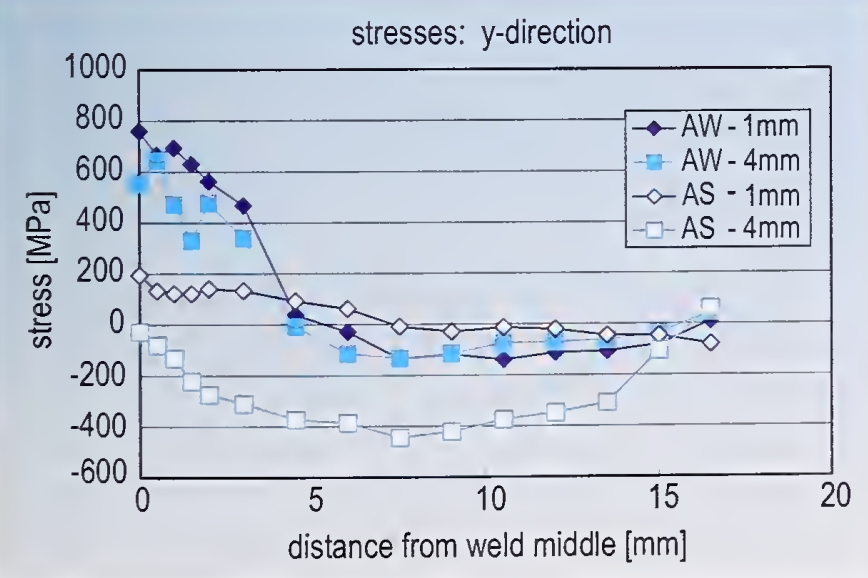

FIGURE 4. Residual stresses $\sigma_{y y}$ (parallel to the weld) before and after the heat treatment. tions. Further investigations are under way to clarify this effect for larger depths.

The stresses parallel to the weld (Fig. 4) are usually the highest and the most dangerous stresses because they reach the yield limit (around $600 \mathrm{MPa}$ ). If the weld direction is oriented such that the weld line is parallel to the main stress axis - i.e., radially for a rotating blade - then further loading would exceed the flow stress and cause a dangerous local weakening of the turbine blade. Here, the effect of the heat treatment is most beneficial because almost complete stress relief is achieved.

The stress relief effect of the heat treatment extends also to the microstresses between sub-grains by removing the dislocations created by the plastic deformation in the weld region. While direct observations of dislocations are not available, we can use the full width at half maximum (FWHM) of the measured integrated strain distributions as a measure for the dislocation density (see Fig. 5).

Figure 5 shows clearly that substantial plastic deformation exists only in the weld itself $(0 \ldots 1.0 \mathrm{~mm})$ and within a very narrow zone of about $0.5 \ldots 1.0 \mathrm{~mm}$ in the base material. This effect vanishes completely after the heat treatment. These results demonstrate that a heat treatment after welding is essential for avoiding detrimental effects to the mechanical properties of the turbine blade.

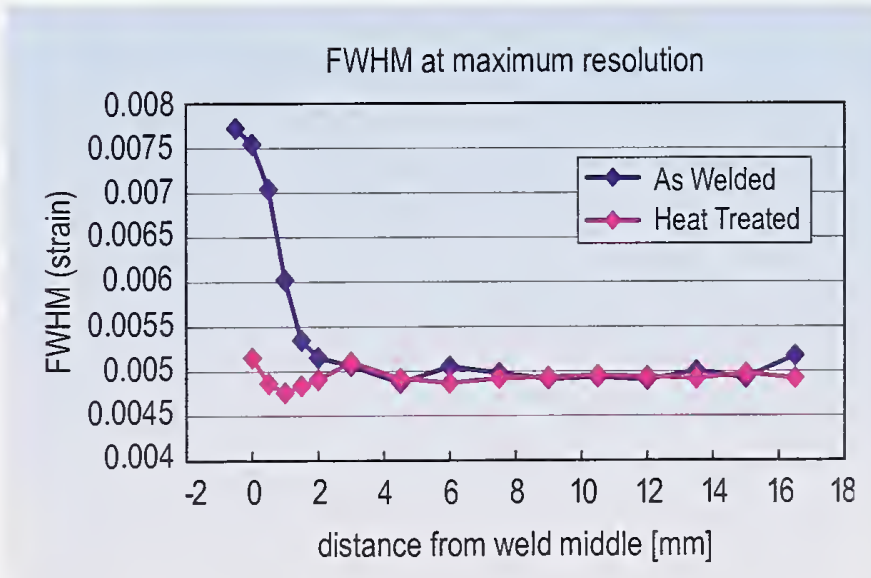

FIGURE 5. FWHM at the best instrument resolution before and after heat treatment. These results are representative for all measured depths. 


\section{EXPGUl: Smoothing the Path to Powder Crystallography}

D ack in the dark ages of computational crystallography, crystallographers were expected to be able to modify their computer programs to suit the computation at hand. Most crystallographers could create programs to convert data between different formats, and felt comfortable reading through a program's source code when the program documentation did not adequately explain a topic. (There is disagreement on when this ended, some say the 1950s, others say the 1980 s or even the early $21^{\text {st }}$ century!)

The advent of desktop computing has also introduced a new paradigm of computing — the graphical user interface (GUI). Visual analogies to physical devices (such as switches) make operating a GUI intuitive; a good GUI implementation significantly reduces learning time.

Simultaneous with the changes in computing, crystallography has evolved from a technique used exclusively by specialists to one of many tools in the arsenal of chemists, physicists and engineers. To facilitate this, the NCNR is developing GUI-based software for crystallography. There are two goals: to increase the utility of the software for the non-specialist, and to improve the productivity of experts. This allows novices to concentrate on the science and techniques of neutron powder crystallography rather than devote their efforts to learning arcane details of computer software.

The Rietveld refinement technique for crystallographic analysis was adopted within the NCNR immediately after its first publication [1]. The NCNR then produced Rietveld software specifically adapted to the local instrumentation, which is still being improved [2, 3]. However, the General Structure Analysis System (GSAS) package of programs from Los Alamos is also widely used [4]. GSAS has wide applicability. It can be used with virtually any type of neutron or $\mathrm{x}$-ray diffraction data, including both powder and single crystal data. It supports a wide range of geometric and compositional restraints and constraints. While many new features have been added to GSAS on a continual basis, the original text-based user interface has not changed. Novice and occasional users often find the many levels of dialogs cumbersome and difficult to learn.

Work on a GUI for GSAS was begun several years ago, with the modest goal of providing a mechanism for accessing a small portion of the GSAS features. The project, now called EXPGUI, has grown in scope [5]. Most of the commonly used powder diffraction features are now implemented, allowing a complete Rietveld refinement to be performed graphically. Features not implemented in the GUI can still be accessed via the standard GSAS user interface. EXPGUI runs identically on all platforms where GSAS is supported, including both Unix and Windows. In FY2001, the Advanced Technology Program recognized the value of this effort and provided funding to accelerate development.

The GUI is designed to display information and controls in a concise and intuitive fashion. An example screen, shown in Fig. 1, manipulates structural parameters. This figure demonstrates how four atoms that share sites in this material can be selected together, so that their refinement options can be changed as a group. Another screen, shown in Fig. 2, applies constraints that link appropriate parameters in the refinement.

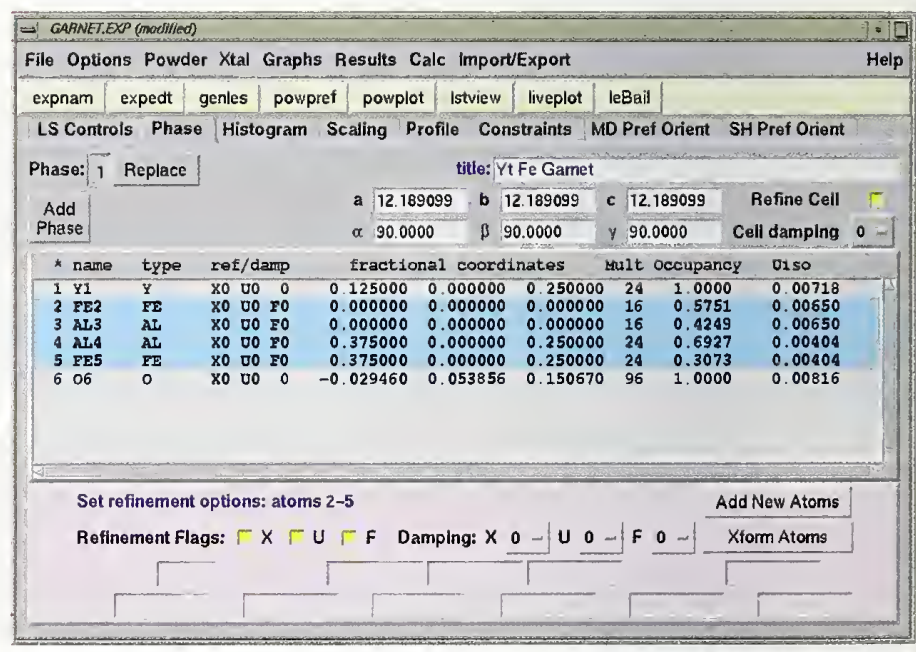

FIGURE 1. Sample screen from EXPGUI, showing where atomic parameters are displayed.

Recent EXPGUI development has been aimed at extending the capabilities of the GSAS package. As an example, when EXPGUI was recently given the ability to import atomic coordinates from files, support was added for CIF, the complex standard crystallographic information file format developed by the International Union of Crystallographers. Further, the import routine was written in a general fashion, so that other formats are easy to add. 




FIGURE 2. EXPGUI screen for entering and display of atomic constraints. In this case, atoms sharing sites are constrained to have the same $U_{i s o}$ values and have fractional occupancies that total to unity.

Where possible, EXPGUI takes advantage of the graphical nature of modern computers to offer scientists better access to refinement tools. Figure 3 shows a graphical display of the observed and computed diffraction pattern. Through a simple set of mouse operations, the user has "zoomed in" on a portion of the data, to better see the poor agreement between observed and computed intensity values, indicating that the structure is not well modeled.

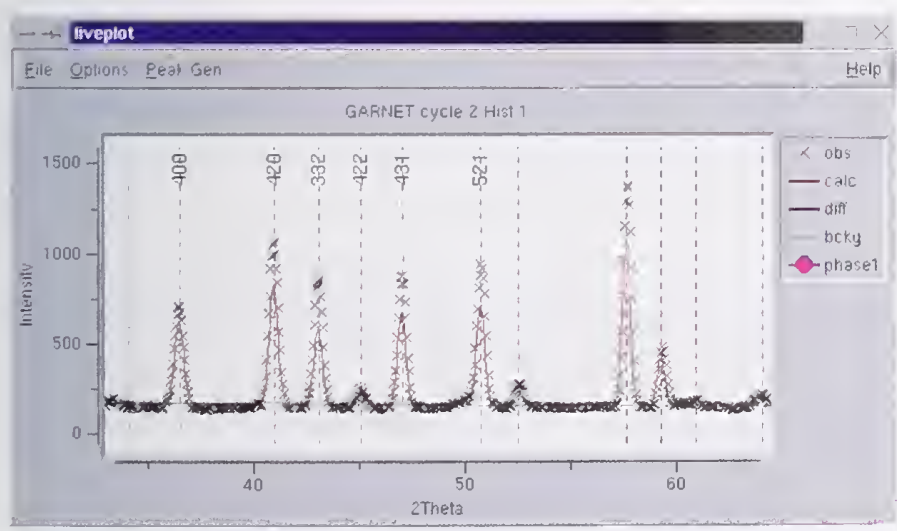

FIGURE 3. Graphical display of fit in EXPGUI. This plot has been "zoomed" in to show details. Several features of this plot, for example the display of reflection indices and the location of the fitted background, were not previously available in GSAS.

This graphical tool also allows the user to see the fitted background and to label the reflection indices — features not previously available within GSAS. Another example is a new tool for background fitting. In most cases, background fitting is nearly automatic, but for some refinements, fitting can be quite difficult. GSAS allows a scientist to define the background either through use of a function with refinable terms or as a spline drawn through a set of fixed points. A new tool in EXPGUI improves GSAS by allowing a user to define where the background curve should occur using a computer mouse. Since many experts agree that fixed background points are a poor way to treat background fitting, EXPGUI offers an augmented feature: a background function can be fit to the user's desired background curve. The terms for this function will be sufficient to obtain an initial model with good agreement to the data. At the end, the background terms can be optimized, to obtain the best possible model. This capability was not previously available in GSAS.

EXPGUI has been widely adopted by Rietveld users. It is used in most major neutron and synchrotron facilities, as well as dozens, if not hundreds, of universities, companies and research centers around the world.

\section{References}

[1] H. M. Rietfeld, J. Appl. Cryst. 2, 65 (1965).

[2] L. W. Finger and E. Prince, National Bureau of Standards, Report No. 852 (1975).

[3] B. H. Toby, E. Prince, and J. Stalick, unpublished computer program (2000).

[4] A. C. Larson and R. B. Von Dreel, Los Alamos National Laboratory, Report LAUR 86-748, (2000).

[5] B. H. Toby, J. Appl. Cryst. 34, 210 (2001). 


\section{Neutron Diffraction Contributes to Improving the Fatigue Life of Bridges}

W elded attachments to large-scale structures such as bridges can limit the serviceability and prevent effective use of high strength steels. To avoid this, it is desirable to enhance the fatigue resistance of common attachment details such as transverse stiffeners, cover plates, gusset plates and other welded details that, at the weld toe, are often the initiation point for crack growth.

Enhancement of fatigue resistance of welded joints by plastic deformation of the surface and by improvement of weld toe characteristics is well established $[1,2]$. It is known that conventional improvement techniques such as grinding, shot peening, air hammer peening, gas tungsten arc re-melting, and use of improved welding consumables can improve fatigue resistance of welded details [3]. However, these procedures are time-consuming, and can be inefficient and environmentally unfriendly. Ultrasonic Impact Treatment (UIT) offers an alternative means to avoid the negative aspects of other methods [4].

The post-weld enhancement of welded details by UIT, developed by Statnikov and co-workers [4], involves deformation treatment of welds by impacts at ultrasonic frequency on the weld toe surface. The objectives of the treatment are to introduce beneficial compressive residual stresses at the treated weld toe zones, and to reduce stress concentration by improving the weld toe profile. UIT is claimed to involve a complex combination of strain hardening, reduction in welding strain, relaxation of residual stress, and a reduction in operating stress concentration, thereby achieving a deeper cold-worked metal layer. However, virtually none of the effects ascribed to UIT have been confirmed by appropriate measurements.

Here we highlight neutron diffraction measurements at the NCNR that have contributed new insights into how UIT actually affects residual stresses in the weld region around a full-scale cover plate for a steel girder. In Fig. 1 a diagram is shown of a typical I-beam fatigue-tested at the Center for Advanced Technology for Large Scale Structures (ATLSS) at Lehigh University. In the initial phase of the NCNRLehigh collaboration, neutron diffraction was used to determine the effect of UIT on a test flange plate by measuring before and after the treatment by UIT. The test plate consisted of a cover plate having a $12.5 \mathrm{~mm}(0.5 \mathrm{in}$.) end fillet weld attaching it to a base plate. This kind of cover- plate detail is often used in steel bridges but has a low level of fatigue strength compared to other welded configurations. The geometry and dimensions of the welded detail are the same as in the full-scale fatigue test beams of Fig. 1. Both base plate and cover plate are steel (A572 Gr.50).

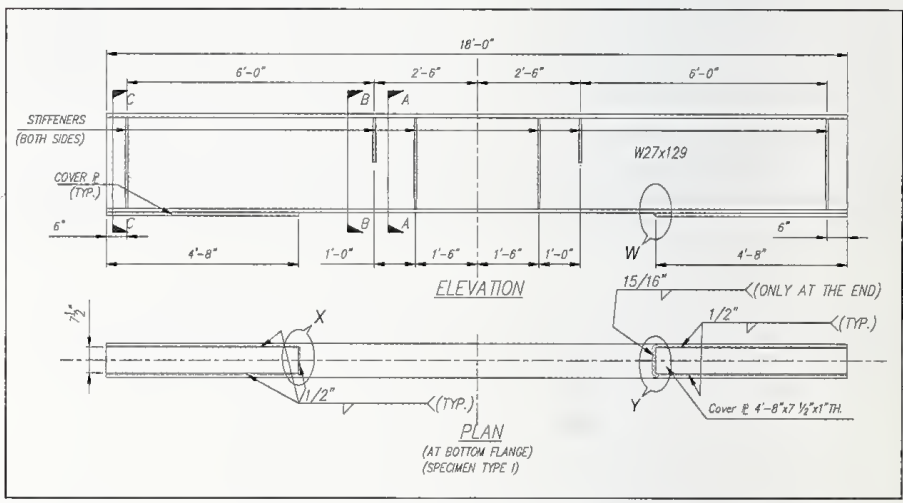

FIGURE 1. Partial details of one of the beam specimens being fatiguetested at the ATLSS facility. The cover plate location is shown as "W" and " $Y$ " on the right side.

Neutron diffraction measurements were made in a mesh, mostly near the weld toe, as indicated in Fig. 2. Overall, residual stresses were determined at 30 positions in the weld region, before and after UIT. Gauge volumes of $3 \mathrm{x}$ $3 \times 3 \mathrm{~mm}^{3}$ (interior) and $2 \times 2 \times 2 \mathrm{~mm}^{3}$ (near surface) were used. The unstressed $d$-spacing, $d_{0}$, was determined from a small piece of the starting material. The depth dependence of $X$-direction stresses is shown in Fig. 3.

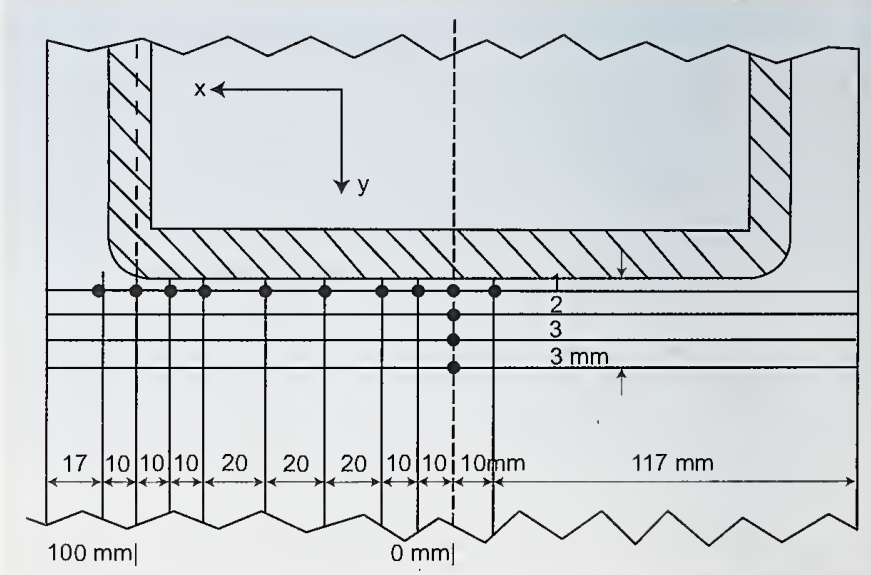

FIGURE 2. Top view of a flat test plate: cover plate (inner rectangular segment), weld (hatched area), and base plate underneath. Part of measurement mesh is shown by dots. 
H. J. Prask, T. Gnäupel-Herold ${ }^{1}$, and V. Luzin²

NIST Center for Neutron Research

National Institute of Standards and Technology

Gaithersburg, MD 20899-8560 and

${ }^{1}$ University of Maryland $\quad{ }^{2}$ State University of New York

College Park, MD 20742 Stony Brook, NY 11794
J. W. Fisher and Xiaohua Cheng

Department of Civil and Environmental Engineering

Lehigh University

Bethlehem, PA 18015-4793

\section{$S_{x x}$ vs. depth at $x=0$}

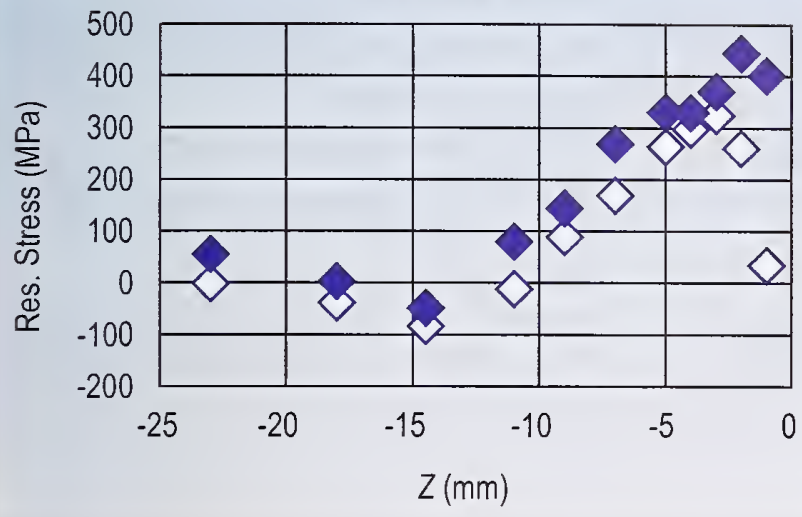

FIGURE 3. $X$-direction residual stresses vs. depth at the weld center. Solid symbols are pre-UIT. Uncertainties are typically $\pm 20 \mathrm{MPa}$.

The near-surface residual stresses determined, nominally $1 \mathrm{~mm}$ below the actual surface, are shown in Fig. 4. Both the anisotropy of stresses around the untreated weld metal, and the additional anisotropy introduced by UIT are seen. Before UIT, the stresses just beneath the weld toe are comparable and highly tensile in the $X$ and $Y$ directions. These stresses reach and exceed the minimum yield stress (315 MPa) of base plate material, whereas those normal to the surface are generally only $\approx 100 \mathrm{MPa}$ tensile. After UIT the $X$-direction stresses become compressive, changing in magnitude by about $400 \mathrm{MPa}$. The $Y$-direction stresses are reduced by $\approx 150 \mathrm{MPa}$ or less but remain in tension except at one point. The normal stresses (Z-direction) are essentially unchanged. The UIT effect on $X$-direction stresses (Fig. 3 ) is significant only near the plate surface, but reaches deeper than, for example, shot peening. The depth dependences of the $Y$ - and $Z$-direction stresses were also obtained.

To our knowledge this is the first determination of the triaxial stress distribution in the vicinity of a weld treated with UIT. Having established the effects of UIT stress-relief on a test flange plate, ongoing neutron diffraction studies in this collaboration are now focusing on the mapping of stress gradients within and near weld joints in sections of actual fatigue-tested I-beams.

\section{References}

[1] J. W. Fisher, K. H. Frank, M. A. Hirt, and B. M. McNamee, Effect of Weldments on the Fatigue Strength of Steel Beams, NCRP Report 102, Highway Research Board, Washington, DC, 1970.

[2] J. W. Fisher, P. A. Albrecht, B. T. Yen, D. I. Klingerman, and B. M. McNamee, Fatigue Strength of Steel Beams with Transverse Stiffeners and Attachments, NCHRP Report 147, Highway Research Board, Washington, DC, 1974.

[3] J. W. Fisher, H. Hausammann, M. D. Sullivan, and A. W. Pense, Detection and Repair of Fatigue Damage in Welded Highway Bridges, NCHRP Report 206, Transportation Research Board, Washington, DC, 1979.

[4] Reviewed in Statnikov, Esh, Applications of Operational Ultrasonic Impact Treatment (UIT) Technologies in Production of Welded Joints, Welding in the World 44, 11 (2000).
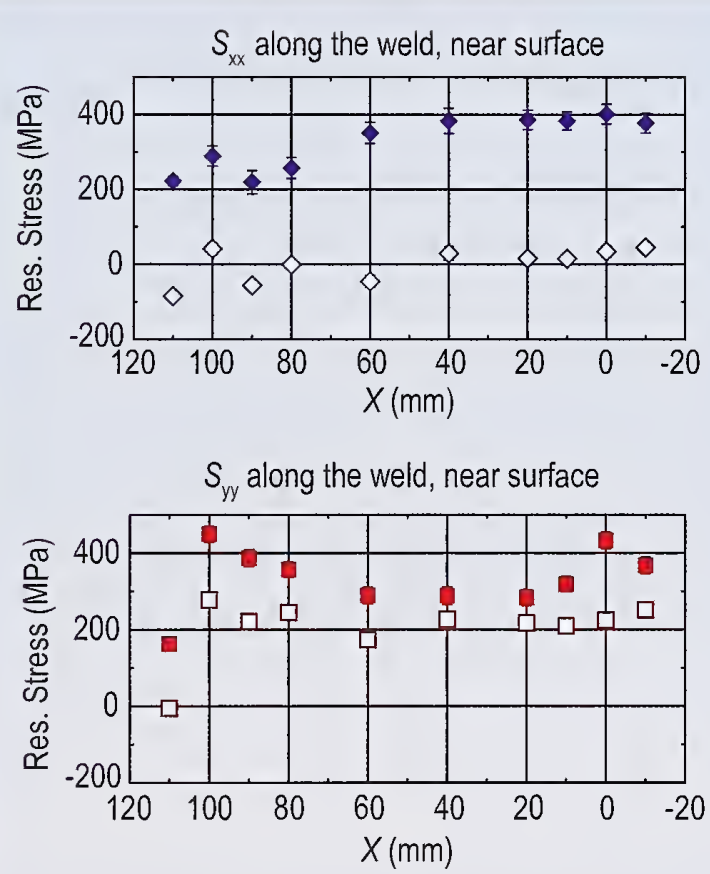

$S_{z z}$ along the weld, near surface

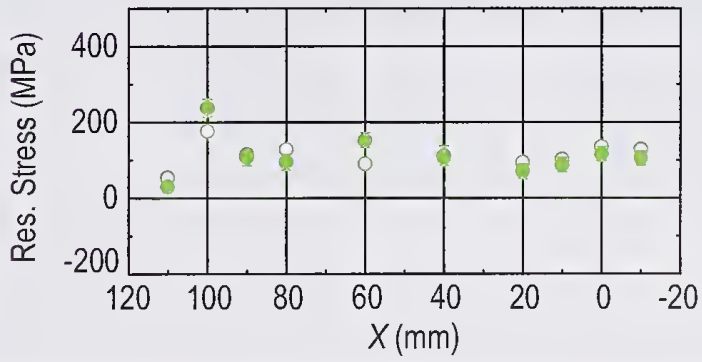

FIGURE 4. The residual stresses determined by neutron diffraction for the "near-surface" points. Uncertainties due to counting statistics are typically about $\pm 20 \mathrm{MPa}$. Solid symbols are before UIT. 


\section{Capillary Neutron Lens for Prompt Gamma Activation Micro-Analysis}

$\mathrm{G}$ lass polycapillary fibers with hundreds of hollow micrometer-wide channels have been used for guiding and changing the direction of slow neutrons [1]. The use of a monolithic lens to focus neutron beams for prompt-gamma activation analysis (PGAA) of small samples has been explored earlier [2]. In addition, the converging nature of a focused beam can provide magnified images for neutron radiography [3]. We have combined these two uses of the focused beam from a monolithic lens and have implemented an alignment procedure for PGAA aided by neutron imaging.

The measurements have been performed at the end of the NG-0 beam line at the NCNR [4]. The average wavelength of the incident beam with a Be filter is about $0.6 \mathrm{~nm}$. Neutrons are imaged with a camera that consists of a $\mathrm{ZnS}(\mathrm{Ag}) / 6 \mathrm{LiF}$ scintillator coupled with a CCD camera with an average pixel size of $50 \mu \mathrm{m}$. The gamma-ray spectrometry system [5] consists of a p-type germanium photon detector with a virtual pulser "loss free" counting module. The two monolithic lenses used in this study are both fabricated from a bundle of capillaries that is $50 \mathrm{~mm}$ long and with a hexagonal cross section $10 \mathrm{~mm}$ wide (flat-to-flat) at the entrance and tapered to $3 \mathrm{~mm}$ at exit. The distance from the exit to the focus is $8.8 \mathrm{~mm}$ for lens A and $9.8 \mathrm{~mm}$ for lens B. Each lens is installed on $x-y-z$ translation stages with two directions of tilt motion. The experimental layout is shown schematically in Fig. 1.

An optimum focal spot from lens $\mathrm{A}$ is recorded with the imaging detector. Line profiles across the focal spot in

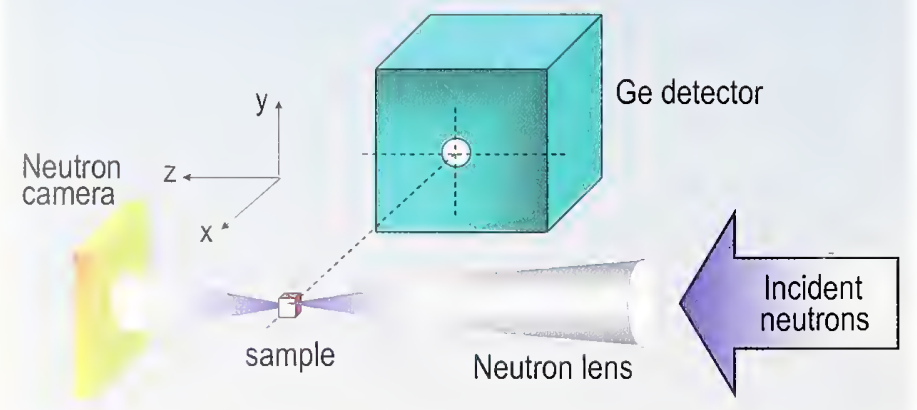

FIGURE 1. Schematic experimental layout. both the horizontal and vertical directions are taken to determine the intensity distribution and the FWHM (H: $87 \mu \mathrm{m}, \mathrm{V}: 100 \mu \mathrm{m}$ ) of the focus (Fig. 2 a). The gain in neutron current density within the $100 \mu \mathrm{m} \times 100 \mu \mathrm{m}$ area is $46 \pm 2$, and within a $50 \mu \mathrm{m} \times 50 \mu \mathrm{m}$ area it is $71 \pm 5$. Images and their sizes and intensity as a function of $z$ are shown in Fig. 2b.

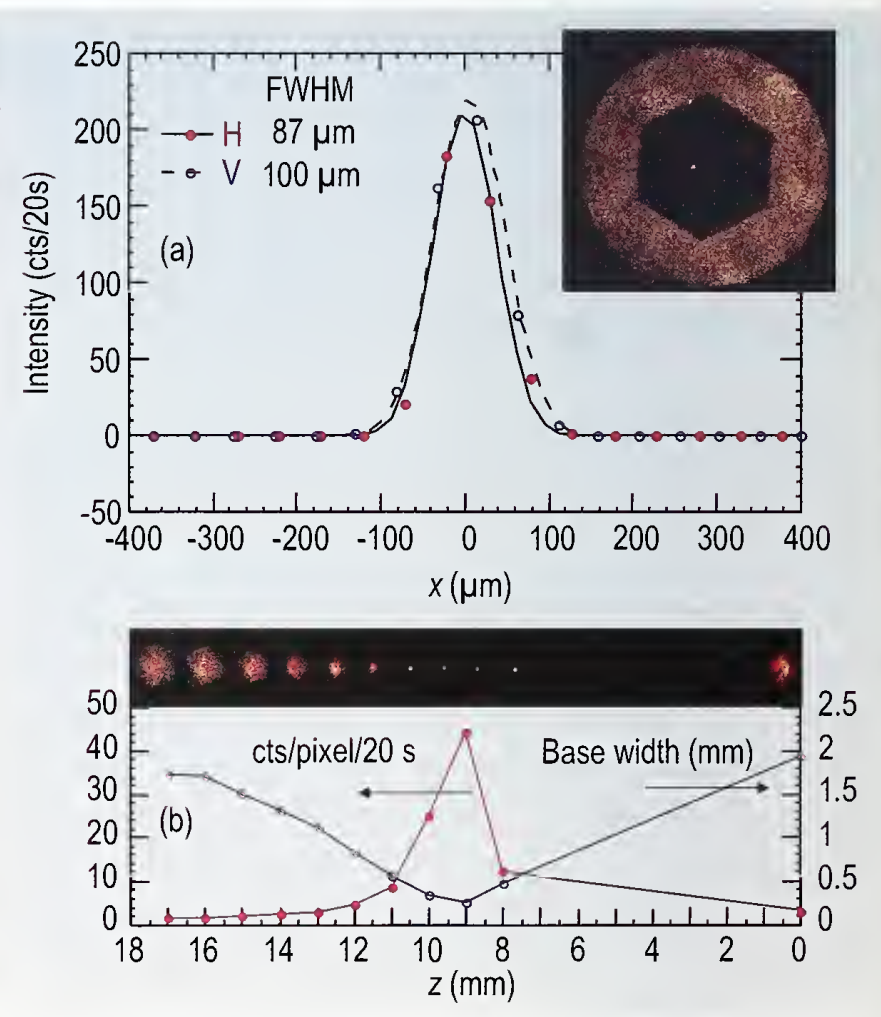

FIGURE 2. (a) Line profiles of the focal spot intensity in the horizontal and vertical directions. Insert: Focused beam image - the focus is near the center of the hexagonal shadow cast by the lens entrance surrounded by the background of unguided neutrons. (b) Intensity and base width of the spot along the beam path, and images of the focused beam "foot print." A linear fit to the region between $10 \mathrm{~mm}$ and $16 \mathrm{~mm}$ gives a slope of 0.233 radians, or a $13.3^{\circ}$ convergence angle.

The focused beam creates a neutron "point source," with neutrons departing the focus within a cone of about $13^{\circ}$. Any neutron-absorbing object within the path of the divergent beam will cast a magnified shadow at some further distance. We make use of this effect in order to align a small sample, a Gd foil (thickness $25 \mu \mathrm{m}$ ) of size $2 \mathrm{~mm}$ x $0.9 \mathrm{~mm}$, at the focus for PGAA (Fig. 3a). We vary the distance $\mathrm{L}_{1}$ between the sample and the lens focus by translating the lens 
H. H. Chen-Mayer, G. P. Lamaze

D. F. R. Mildner and R. Zeisler

NIST Center for Neutron Research, and

Analytical Chemistry Division

Chemical Science and Technology Laboratory

National Institute of Standards and Technology

Gaithersburg, MD 20899-8395
W. M. Gibson

X-Ray Optical Systems, Inc.

Albany, NY 12203

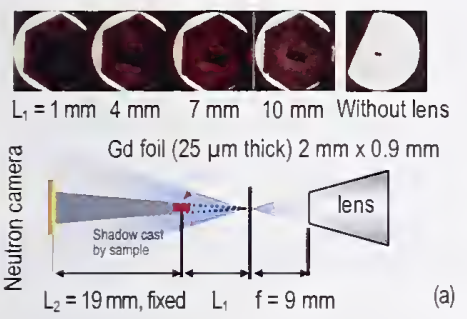

FIGURE 3. (a) Magnified image of the sample at various values of $L_{1}$. The image of the sample without the lens is also shown for comparison. (b) Prompt gamma spectrum for $\mathrm{Gd}$ (182 keV) and for In (186 keV) measured with the lens, and the corresponding image of the sample.

along the direction of the beam. The magnified image enables a better alignment of the sample with respect to the focus. From the earlier measurement of the lens focal point (Fig. 2), we determine the location of the focus with respect to the large hexagonal shadow cast by the lens entrance. We then move the lens transversely such that the image of the sample is now superimposed on the image of the focal point. This guarantees that the focal point is indeed on the sample. Once the image is centered, the lens is translated to $\mathrm{L}_{1}=0$ such that the focus is on the sample, ready for prompt gamma measurements.

At the center of the same Gd sample described above, there is an additional indium foil of about $1 \mathrm{~mm} \times 1 \mathrm{~mm}$ in cross section, in contact with the Gd strip. The prompt gamma measurement without the lens gives a peak intensity for Gd at $182 \mathrm{keV}$ of 2.1 counts per second (cps), but the In peak at $186 \mathrm{keV}$ is not visible even for a 20 min measurement. When the lens is used in a 10 min measurement, not only has the Gd peak intensity increased, but the In peak has also become visible (Fig. 3b). Since the Gd piece is much larger than the focal spot size, the gain in sensitivity for Gd is only a factor of 4 . The gain for In is not estimated.

We have also used lens B for an even smaller sample, a Gd piece of $128 \mu \mathrm{m} \times 103 \mu \mathrm{m} \times 25 \mu \mathrm{m}$ in size and $2.59 \mu \mathrm{g}$ in mass (effective mass $\approx 1 \mu \mathrm{g}$ taking into account selfabsorption). The images from an optical microscope and from neutron measurements are shown in Fig. 4a. Without the lens, the neutron camera cannot resolve the $100 \mu \mathrm{m}$ particle. The gamma intensity with the lens is $17.4 \mathrm{cps}$ with

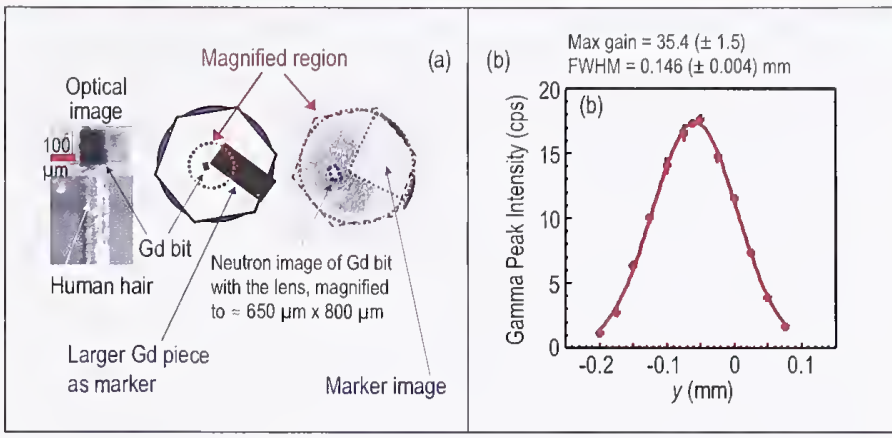

FIGURE 4. (a) Optical image of the Gd piece in comparison with a strand of human hair (left), and neutron image using lens $B$. The large solid rectangle is a larger $\mathrm{Gd}$ strip marker to help locate the smaller sample more easily. (b) Gd peak intensity produced by a y-scan of the lens.

a $1.6 \% 1 \sigma$ counting statistics after 1 hour of counting, and with the direct beam 0.506 cps $(3.9 \% 1 \sigma)$ after 19.5 hours. The gain, given by the ratio of the two count rates, is $34 \pm$ 1.4. A scan of the lens in the vertical direction yields a peak, given by the convolution of the sample width with the beam width, as shown in Fig. 4b. The FWHM of the peak is 148 ( \pm 1.5$) \mu \mathrm{m}$, correctly corresponding to the $100 \mu \mathrm{m}$ samplewidth and the $100 \mu \mathrm{m}$ beam-width added in quadrature.

The effort presented here is a step toward providing quantitative elemental information to traditional neutron radiography. This new tool will provide a unique capability in non-destructive analysis for industrial materials. In the future, the system will be automated to scan, giving a more efficient probe for microanalysis with neutron beams.

\section{References}

[1] M. A. Kumahkov and V. A. Sharov, Nature 357, 390 (1992), and H. Chen, R. G. Downing, D. F. R. Mildner, W. M. Gibson, M. A. Kumakhov, I. Yu. Ponomarev, and M. V. Gubarev, Nature 357, 391 (1992).

[2] V. A. Sharov, Q. F. Xiao, I. Yu. Ponomarev, D. F. R. Mildner, and H. H. Chen-Mayer, Rev. Sci. Instrum. 71, 3247 (2000).

[3] K. M. Podurets, D. F. R. Mildner, and V. A. Sharov, Rev. Sci. Instrum. 69, 3541 (1998).

[4] D. F. R. Mildner, H. H. Chen-Mayer, G. P. Lamaze, and V. A. Sharov, Nucl. Instrum. \& Meth. A413, 341 (1998).

[5] R. Zeisler, G. P. Lamaze, H. H. Chen-Mayer, J. Radioanal. Nucl. Chem. 248, 35 (2001) 


\section{Giant Anharmonicity and Electron-Phonon Mediated Superconductivity in $\mathrm{MgB}_{2}$ at $39 \mathrm{~K}$}

$\mathbf{T}$ he recent surprise discovery of superconductivity in $\mathrm{MgB}_{2}$ at $39 \mathrm{~K}$ has stimulated a great deal of research on this intercalated graphite-like system (see Fig. 1). Sparked by this discovery, we set out to unlock the structural secrets and, in particular, to reveal the origin of the high $T_{c}$ in $\mathrm{MgB}_{2}$ : an electron-phonon or other exotic mechanism? To answer this fundamental question, we calculated $T_{c}$, its pressure dependence, and its isotope effect from the electronic band structure and lattice dynamics of $\mathrm{MgB}_{2}$ using density functional theory within the generalized gradient approximation [1-3].

Figure 2 shows that the features in the calculated phonon density of states (DOS) are in excellent agreement with the neutron data (GDOS), giving confidence that the calculations provide a sound description of the physical properties of the system. The DOS consists of two bands of phonons, one below $40 \mathrm{meV}$ corresponding primarily to acoustic phonon modes, and one above $50 \mathrm{meV}$ that mostly involves the boron motions. Inspection of the calculated phonon-dispersion curves that make up the high-energy band in the DOS reveals that the in-plane boron phonons (as depicted in the inset to Fig. 3) are very anharmonic. To demonstrate this, in Fig. 3 we plot the total energy as boron atoms move according to one of these zone-center in-plane phonons with $E_{2 g}$ symmetry. The potential indicates a very strong anharmonic term. Numerically solving the

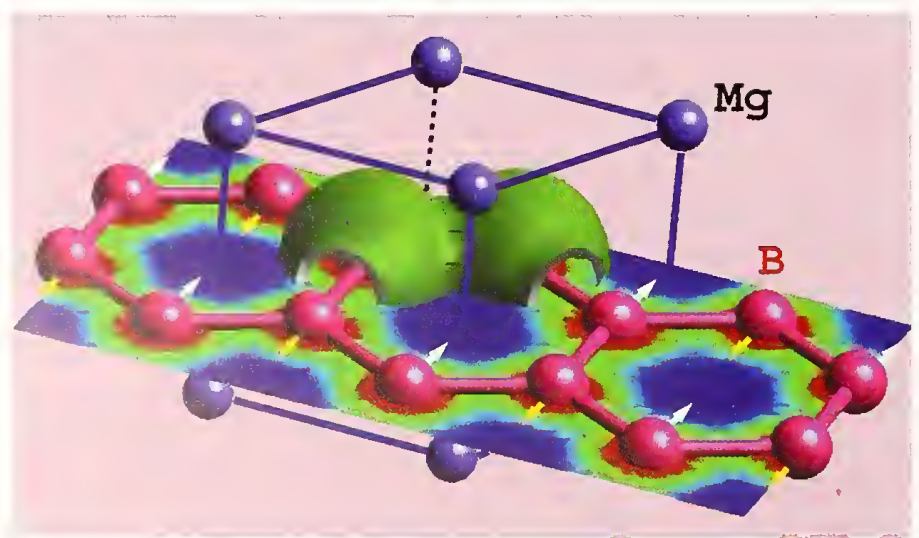

FIGURE 1. The crystal structure of $\mathrm{MgB}_{2}$ consisting of $\mathrm{B}$ and $\mathrm{Mg}$ hexagonal layers. The in-plane boron modes (shown by arrows) are strongly coupled to the boron $p_{x, y} \sigma$ bands shown as the green contour and isosurface plots.
Schrödinger equation for this anharmonic potential yields a phonon energy of $\hbar \omega\left(E_{2 g}\right)=74.5 \mathrm{meV}$, a $25 \%$ enhancement over the harmonic value of $60.3 \mathrm{meV}$. This value is in good agreement with recent Raman measurements. The giant anharmonicity revealed gives the first hint that the in-plane modes are strongly coupled to the $p_{x y} \sigma$ bonding orbitals of boron, as shown schematically in Fig. I. This coupling is also evident from the splitting of the boron $\sigma$ bands (red lines) with the $E_{2 g}$ phonons (see Fig. 3). Note that the other bands are not affected by the $E_{2 g}$ phonons.

The splitting of the boron $\sigma$ bands, when averaged over the Fermi surface, gives an electron-phonon (EP) coupling constant $\lambda \approx 1$. Using this value in the McMillan expression for $T_{c}$ with $\omega\left(E_{2 g}\right)$ and taking a typical value for the Coulomb repulsion $\mu^{*}=0.15$, we obtain a $T_{\mathrm{c}}$ of $39.4 \mathrm{~K}$, in excellent agreement with experiments. We also solved the Schrödinger equation for the potential shown in Fig. 3 for different boron masses and obtained $\omega\left(E_{2 g}\right)=291.8 M^{-0.575}$ and $\lambda=0.6151 M^{0.169}$, which yields the $T_{\mathrm{c}}-M$ curve shown in Fig. 4 and a boron isotope effect $\alpha=0.21$, in good agreement with the experimental value of $0.26 \pm 0.03$.

Since the pressure dependence of $T_{\mathrm{c}}$ puts a stringent test on any theory of superconductivity, we repeated the calculations of phonons and electronic band structures discussed above for isotropic, uniaxial (along $c$-axis), and biaxial (in the $a b$-plane) pressures [3]. We find that while

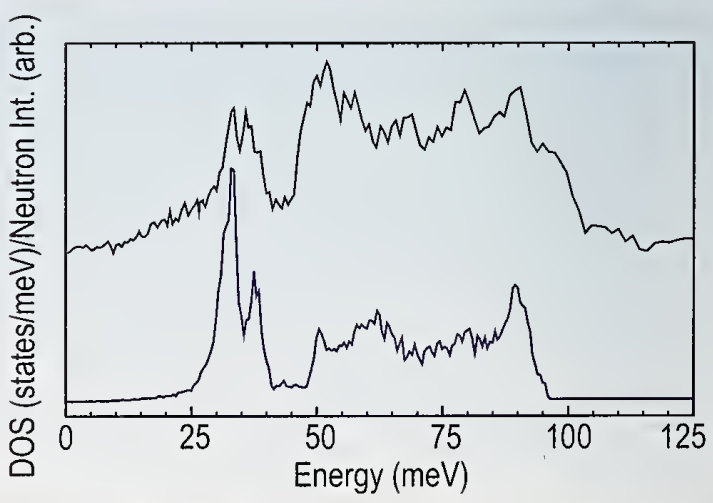

FIGURE 2. Generalized (top) and the calculated (bottom) phonon density of states. The intensities of the peaks do not agree well because in DOS $\mathrm{B}$ and $\mathrm{Mg}$ ions contribute equally while in GDOS they are weighted by neutron cross-sections and inverse masses. 
T. Yildirim

NIST Center for Neutron Research

National Institute of Standards and Technology

Gaithersburg, MD 20899-8562
O. Gulseren

NIST Center for Neutron Research

National Institute of Standards and Technology

Gaithersburg, MD 20899-8562 and

Department of Materials Science and Engineering

University of Pennsylvania

Philadelphia, PA 19104-6202
$\omega\left(E_{2 g}\right)$ increases with increasing pressure, the density of states at the Fermi energy decreases. The EP constant $\lambda$ also shows significant changes with pressure. Inserting all these competitive effects into the McMillan formula yields the pressure dependence of $T_{c}$ shown in Fig. 4.

For isotropic pressure $T_{\mathrm{c}}$ decreases with increasing pressure almost linearly at a rate of $\approx-1.0 \mathrm{~K} / \mathrm{GPa}$, in excellent agreement with the experimental value of $-1.1 \mathrm{~K} / \mathrm{GPa}$. We also predict a cusp in the $T_{\mathrm{c}}-P$ curve around $P \approx 20 \mathrm{GPa}$.
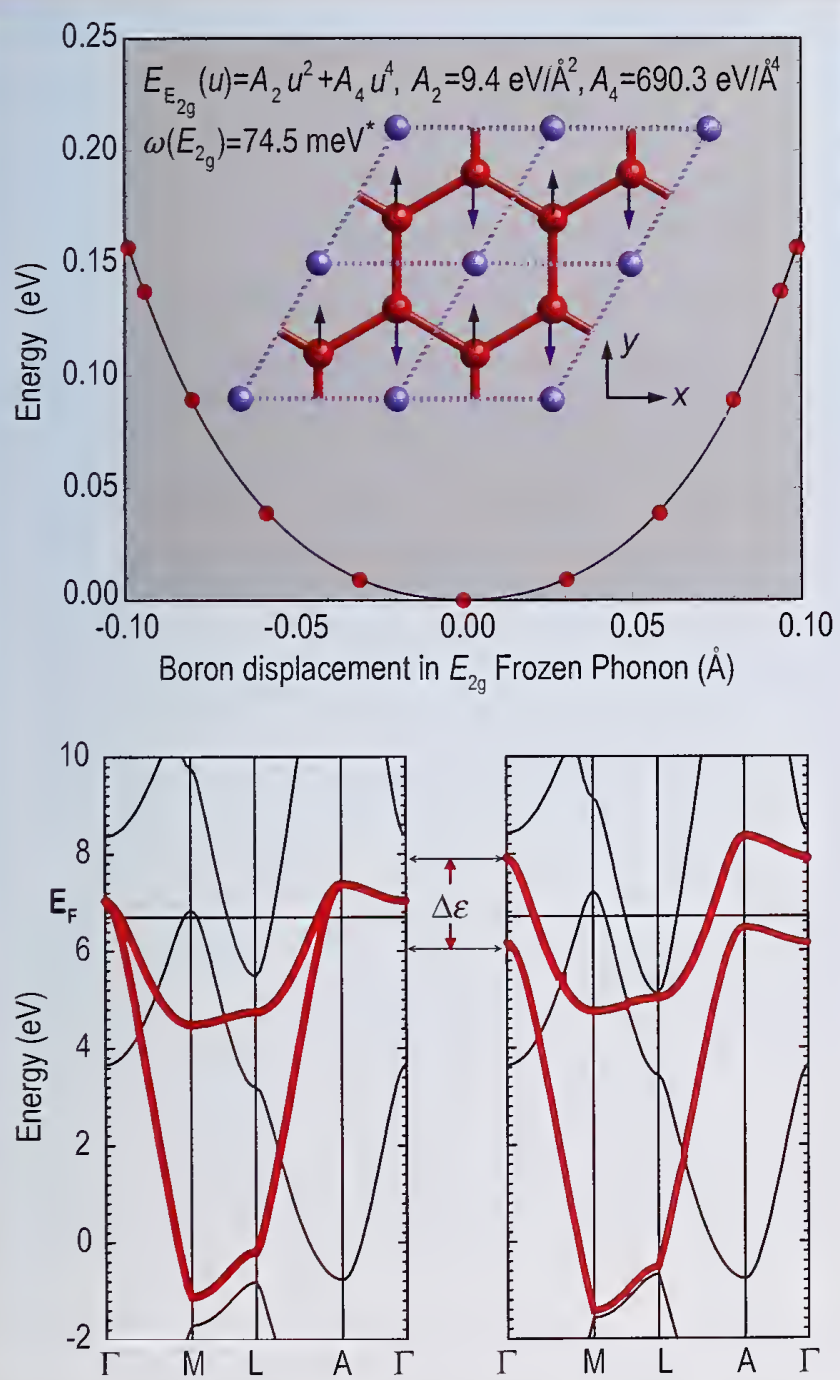

FIGURE 3. Top: Total energy curve as a function of boron displacement for $E_{2 g}$ mode (shown in the inset), indicating a large anharmonic term in the potential. Bottom: Band structure of the undistorted (left) and distorted structures (right) by $E_{2 g}$ phonons $\left(u_{B} \approx 0.06 \AA\right)$. See Ref. 1 for the animation of the zone center phonons and their coupling with the $B \sigma$ bands.
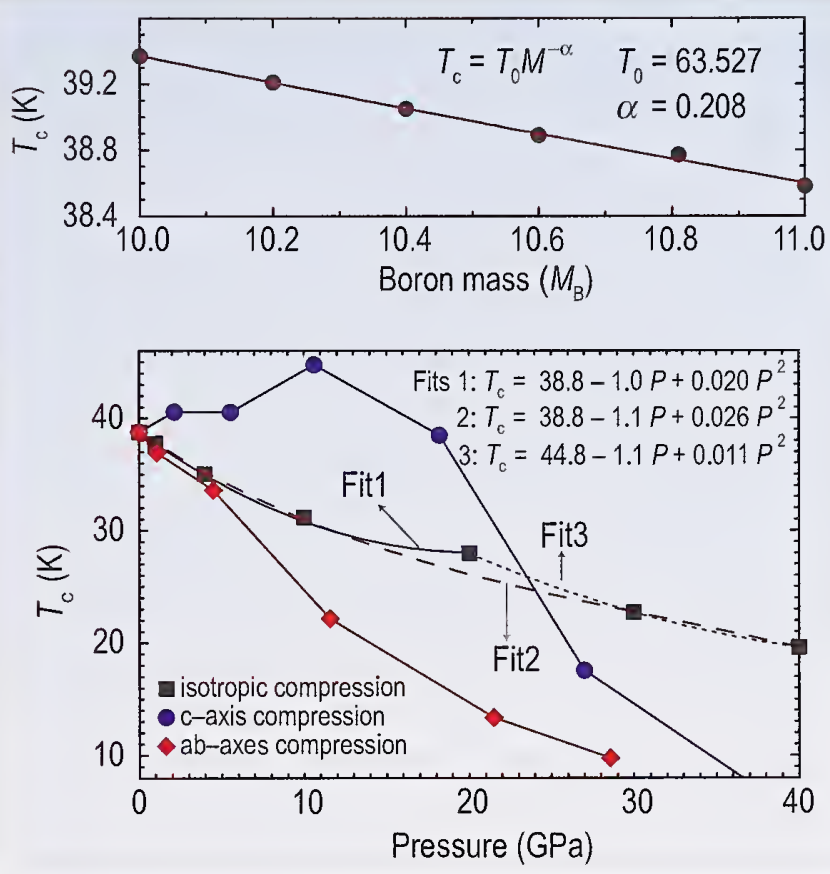

FIGURE 4. Top: Boron mass dependence of the $T_{c}$. Bottom: Pressure dependence of $T_{c}$ as a function of uniform $a b$-axes and $c$-axis compression, respectively.

A similar cusp was recently observed experimentally. Our calculations indicate that $T_{c}$ should increase first and then decrease with increasing $c$-compression, while it should decrease rapidly with $a b$-compression. Hence, when single crystal samples of $\mathrm{MgB}_{2}$ become available, measurements of the $a b$ - and $c$-compression dependence of $T$ should provide a critical test of our theory.

$\mathrm{MgB}$, may be the ultimate BCS s-wave superconductor, with parameters controlling $T_{c}$ fully optimized to yield the highest possible $T_{c}$. However, even if $T_{c}$ cannot be increased further, the low cost, light mass, easy fabrication, nearly isotropic high conductivity of $\mathrm{MgB}_{2}$, which also has a large critical current, will no doubt find many important technological applications in the near future.

\section{References}

[1] For full details of this work, see the website: http://www.ncnr.nist.gov/ staff/taner/mgb2.

[2] T. Yildirim, O. Gulseren, J. F. Lynn, C. M. Brown, T. J. Udovic, Q. Huang, N. Rogado, A. Regan, M. A. Hayward, J. S. Slusky, T. He, M. K. Haas, P. Khalifah, K. Inumaru, and R. J. Cava, Phys. Rev. Lett. 87, 37001 (2001).

[3] T. Yildirim and O. Gulseren, J. Phys.Chem. Solids (in press, 2002) 


\section{Polaron Formation and Colossal Magnetoresistance in Manganites}

$\mathrm{T}$ he recent discovery of huge magnetoresistance effects in the manganese oxide class of materials (such as $\left.\mathrm{La}_{l-x} \mathrm{~A}_{x} \mathrm{MnO}_{3}(\mathrm{~A}=\mathrm{Sr}, \mathrm{Ca}, \mathrm{Ba})\right)$ has rekindled intense interest in these systems for three reasons. One is that this "colossal magnetoresistance" (CMR) offers potential in a number of technologies, such as for read/write heads in magnetic recording media, sensors, and spin-polarized electronics. The second reason is that these materials are interesting from a fundamental viewpoint. In contrast to traditional isotropic ferromagnets such as $\mathrm{Fe}, \mathrm{Co}, \mathrm{Ni}$, and $\mathrm{EuO}$ where the spin system is isolated from the lattice, in the manganites the charge, spin, and lattice degrees of freedom are strongly coupled together, leading to a delicate balance of interactions that gives rise to a rich variety of physical phenomena of current interest in condensed matter science. These include a metal-insulator transition that is juxtaposed with ferromagnetism, charge ordering, orbital ordering, polaron formation, electronic phase separation, and spin and charge stripes. The third reason is because of the similarity to the high $T_{c}$ cuprate oxides. Recent progress in our understanding of the cuprates has provided insights into the manganites, and a deeper understanding of the fundamental properties of the manganites will surely elucidate the shared concepts underlying both classes of materials.

The electrical conductivity in these manganites is directly connected to the magnetic system through the double exchange mechanism, while the Jahn-Teller distortion of the $\mathrm{Mn}^{3+} \mathrm{O}_{6}$ couples the magnetic and lattice systems, resulting in the electronic, lattice, and magnetic degrees of freedom being intimately intertwined. The ferromagnetic transition then is non-conventional, and typically coincides with a metal-insulator transition to produce CMR. The present measurements on $\mathrm{La}_{0.7} \mathrm{Ca}_{0.3} \mathrm{MnO}_{3}$, which is near optimal doping for $\mathrm{CMR}$, show that polarons form as the ferro- to paramagnetic transition is traversed, self-trapping the carriers. These polarons then "melt" when a magnetic field is applied, freeing the carriers and generating the CMR.

The scattering from a lattice polaron arises from the structural distortion that surrounds a carrier and traps it. Individual polarons generate diffuse scattering around the fundamental Bragg peaks, while polaron correlations will give rise to additional superlattice peaks. We observe both of these effects [1], and in Fig. 1 we show the four satellite

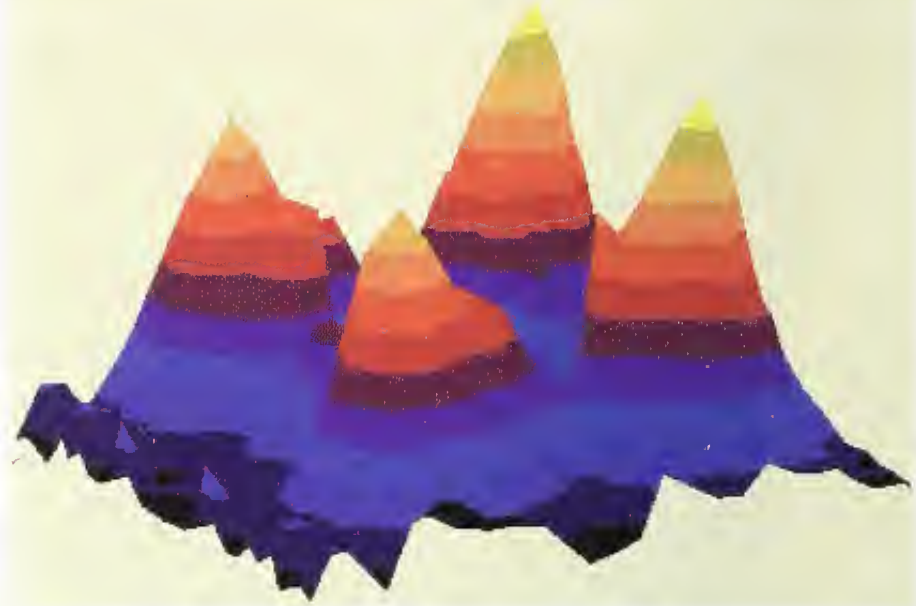

FIGURE 1. Polaron correlation peaks near the 500 Bragg peak at $270 \mathrm{~K}$, just above $T_{c}=255 \mathrm{~K}$.

peaks at $1 / 41 / 40$ positions near the 500 Bragg peak, just above $T_{\mathrm{c}}(255 \mathrm{~K})$. The intrinsic widths of these peaks indicate that the correlations are short range $(\approx 15 \AA)$ in nature, and this range is found to be only weakly temperature dependent. Polarized neutron measurements show that these peaks originate from lattice distortions rather than magnetic scattering. Similar direct evidence for polaron formation and correlations has been observed in the bilayer manganite $\mathrm{La}_{12} \mathrm{Sr}_{1.8} \mathrm{Mn}_{2} \mathrm{O}_{7}$ [2], although the ordering wave vectors and the ratios of diffuse scattering to correlated scattering are different.

A model that can explain both the ordering wave vector as well as the overall behavior of the observed intensities of the short-range correlation peaks is the $\mathrm{CE}$ model developed for the half-doped case. The CE model has an orbitally-ordered Jahn-Teller lattice with charge stripes in the [110] direction, and an antiferromagnetic ground state. Such ordering has been observed for $x \geq 0.5$ at low temperatures. This behavior contrasts with the present ferromagnetic system, where the orbital/charge correlations are short range in nature, occur only above $T_{\mathrm{c}}$, and are not accompanied by any antiferromagnetic correlations. The CE "stripes" are also consistent with stripe formation as seen in other transition metal oxides (nickelates and cuprates).

Of particular interest is how the polaron ordering is related to the metal-insulator transition and the quasielastic 
C. P. Adams ${ }^{1}$ and J. W. Lynn

NIST Center for Neutron Research

National Institute of Standards and Technology

Gaithersburg, MD 20899-8562

and

University of Maryland

College Park, MD 20742
Y. M. Mukovskii, A. A. Arsenov, and D. A. Shulyatev

Moscow Steel and Alloys Institute

Moscow, Russia "central peak" [3] associated with the spin component of the polaron. As we see in Fig. 2, the temperature dependence of the intensity of the charge ordering is nearly identical to the quasielastic magnetic peak, and both follow the resistivity behavior. This is a very strong indication that these properties have a common origin in polaron formation.

A further indication of the strong relationship between charge ordering and resistivity is established by measurements in magnetic fields [1]. In Fig. 3 we see that the intensity of the charge ordering peaks decreases rapidly with increasing field. Thus the polarons "melt" when the magnetization develops and the conductivity increases, either by lowering the temperature (Fig. 2) or increasing the applied field, producing CMR. Identical field dependence has been observed both for the central peak in $\mathrm{La}_{0.7} \mathrm{Ca}_{0.3} \mathrm{MnO}_{3}[1,3]$ as well as the polaron peaks in the bilayer system [2].

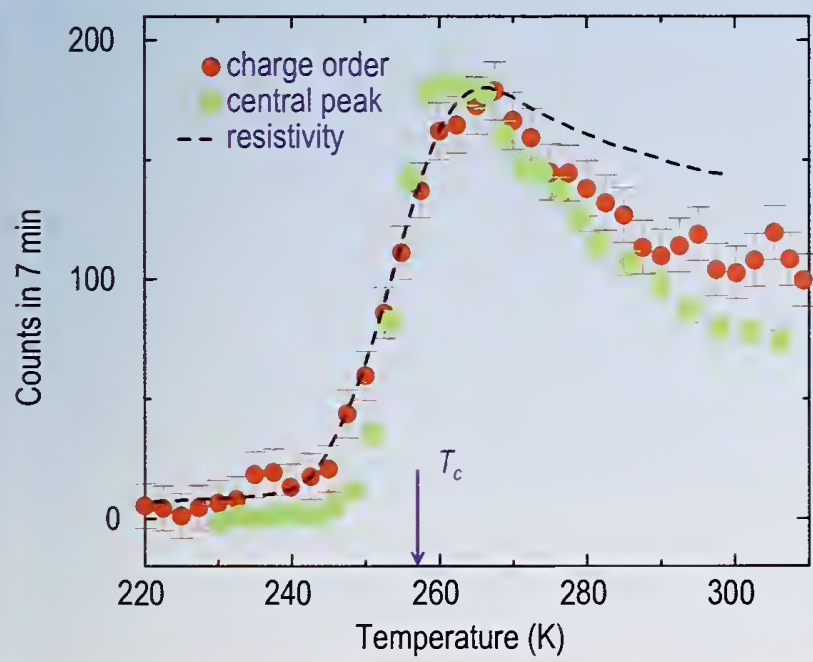

FIGURE 2. Temperature dependence of the intensity of the polaron peak at 3.750 .250 , compared to the magnetic quasielastic central peak, and the resistivity. The data have been scaled vertically so the peak heights match. The similarity of the data indicates a common physical origin.

The above experimental results reveal that both the spin and charge correlations associated with the polarons in $\mathrm{La}_{0.7} \mathrm{Ca}_{0.3} \mathrm{MnO}_{3}$ appear together, and have a very similar temperature dependence. This coincidence may explain the amplified magnetoresistive effects, as well as the absence of conventional magnetic critical behavior. The physical quantities that control the characteristic temperature for polaron formation, including the role that intrinsic inhomogeneities, stress, and phase separation play in their formation, still remain to be elucidated.

\section{References}

[1] C. P. Adams, J. W. Lynn, Y. M. Mukovskii, A. A. Arsenov, and D. A. Shulyatev, Phys. Rev. Lett. 85, 3954 (2000); J. Appl. Phys. 89, 6846 (2001).

[2] L. Vasiliu-Doloc, S. Rosenkranz, R. Osborn, S. K. Sinha, J. W. Lynn, J. Mesot, O. Seeck, G. Preosti, A. J. Fedro, and J. F. Mitchell, Phys. Rev. Lett. 83, 4393 (1999).

[3] J. W. Lynn, R. W. Erwin, J. A. Borchers, Q. Huang, A. Santoro, J. L. Peng, and Z. Y. Li, Phys. Rev. Lett. 76, 4046 (1996); J. Appl. Phys. 81. 5488 (1997).

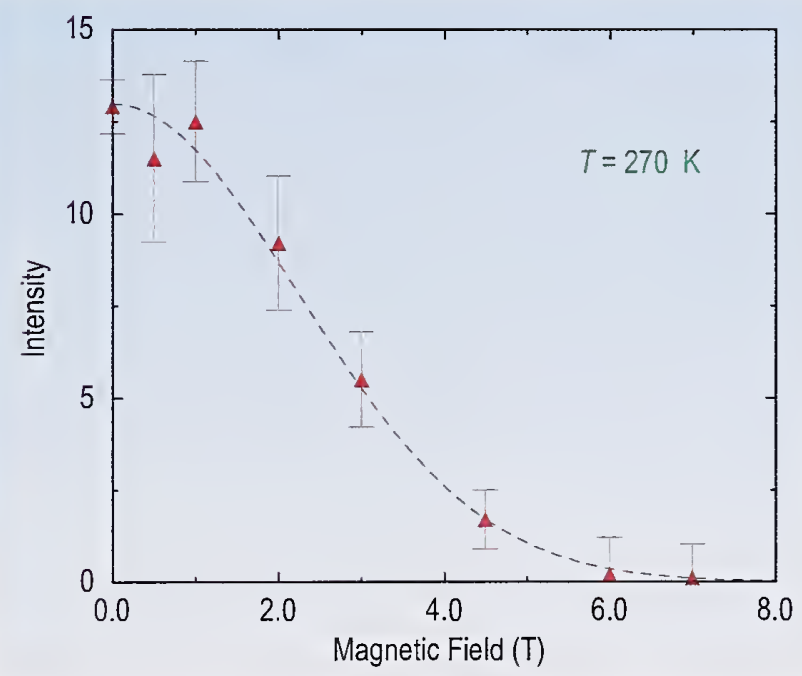

FIGURE 3. Magnetic field dependence of the intensity of the polaron peak, showing that the polarons melt with increasing field. 


\section{Ferromagnetism and Spontaneous Vortex Formation in Superconducting $\mathrm{ErNi}_{2} \mathrm{~B}_{2} \mathrm{C}$}

T he competition between magnetic order and superconductivity has had an interesting history. The first examples of true long range magnetic order coexisting with superconductivity were provided by the ternary Chevrelphase superconductors and related compounds. The magnetic ordering temperatures were all low $(\approx 1 \mathrm{~K})$, indicating that dipolar interactions dominate the energetics of the magnetic system. Similar behavior is found for the rare earth ordering in the hole-doped cuprates [1]. The first systems where exchange clearly dominates the magnetic energetics were provided by the electron-doped cuprates [1], followed by the borocarbides of central interest here [2].

Among these systems, the rare and interesting situation where ferromagnetic interactions are present has attracted special attention because of the competitive nature between the superconducting screening and the internally generated magnetic field. In the Chevrel phase materials this competition gives rise to long wavelength oscillatory magnetic states and/or reentrant superconductivity. Recently, superconducting $\mathrm{ErNi}_{2} \mathrm{~B}_{2} \mathrm{C}\left(T_{\mathrm{c}}=11 \mathrm{~K}\right)$ appeared to develop a net magnetization below $2.3 \mathrm{~K}$, well below the onset of long range spindensity-wave order at $T_{\mathrm{N}}=6 \mathrm{~K}$. We have carried out a comprehensive study of the magnetic order and vortex structure in this material, and directly demonstrated that this transition does indeed correspond to the development of a net atomic magnetization that coexists with superconductivity, and this results in the spontaneous formation of vortices in the system [3].

Superconducting $\mathrm{ErNi}_{2} \mathrm{~B}_{2} \mathrm{C}$ orders magnetically at $6 \mathrm{~K}$ into a transversely polarized spin density wave structure $[2,3]$, with the modulation wave vector $\delta$ along the $a$ axis and the spins along the $b$ direction. A portion of a scan along the $a$ axis is shown in Fig. 1 at three temperatures. The initial ordering is a simple spin density wave as shown in the schematic, with an incommensurate modulation of $\delta \cong 0.55 a^{*}\left(a^{*}=2 \pi / a\right)$. At $2.4 \mathrm{~K}$ additional higher-order peaks become observable as the magnetic structure squares up as indicated schematically at the bottom of Fig. 1. These peaks are all odd-order harmonics as expected for a squarewave magnetic structure.
Below the weak ferromagnetic transition $\left(T_{\mathrm{WFM}}\right)$ at $2.3 \mathrm{~K}$, the data (Fig. 1) indicate that a new series of peaks has developed, which are even harmonics of the fundamental wave vector. Polarized neutron measurements unambiguously establish that both the odd-order peaks and the evenorder peaks are magnetic in origin.

The integrated intensities of the odd- and even-order harmonics are shown in Fig. 2 as a function of $T$. The evenorder peaks abruptly develop below $2.3 \mathrm{~K}$, concomitant with the development of the new (weak) ferromagnetism at $T_{\mathrm{WFM}}$.
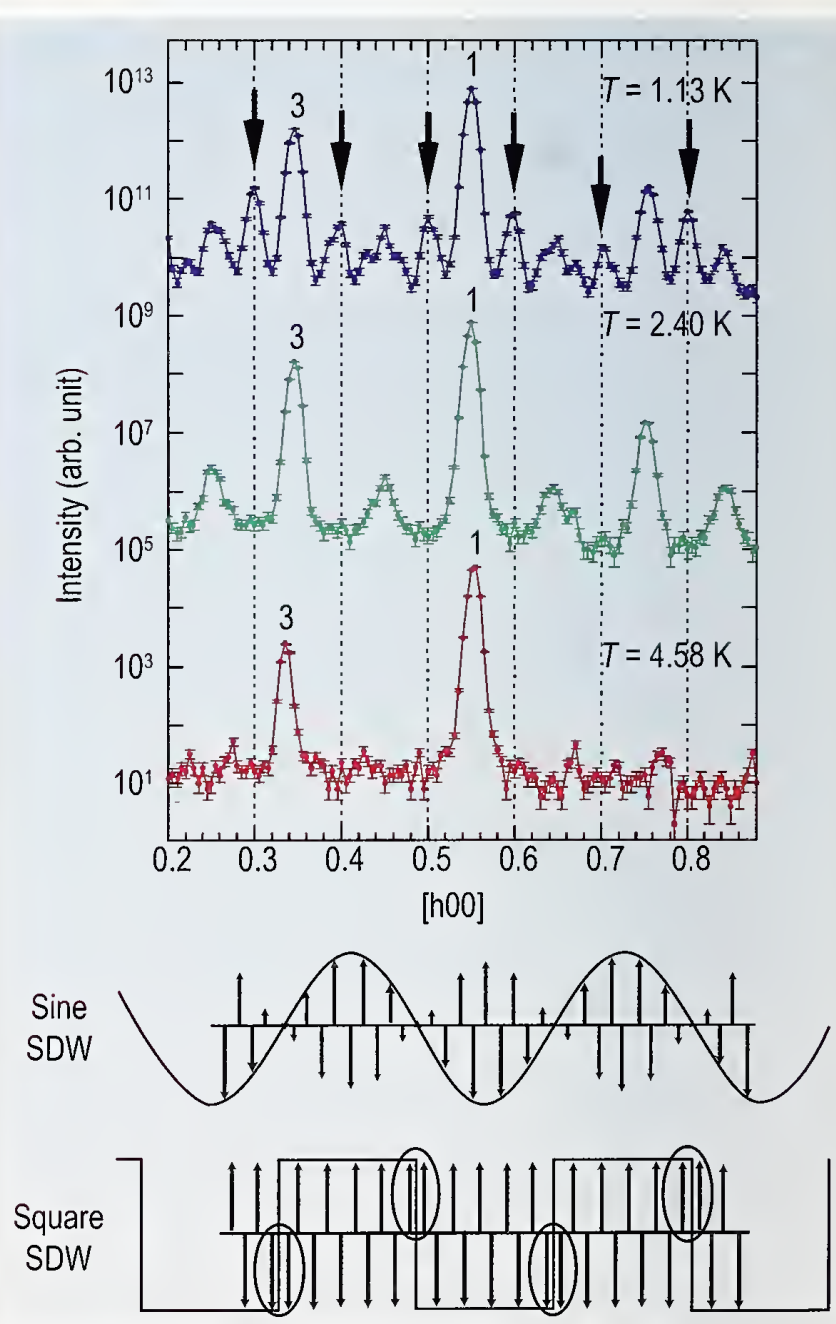

FIGURE 1. Scan along the [h00] direction at $T=1.3 \mathrm{~K}, 2.4 \mathrm{~K}$, and $4.58 \mathrm{~K}$. The data have been offset for clarity. Above the weak ferromagnetic transition at $2.3 \mathrm{~K}$ we observe the fundamental peak at $\approx 0.55$ along with odd-order harmonics, while below a new set of even harmonics develops (arrows). Also shown is a schematic of the spin density wave order for $T$ just below $T_{N}$, and the (undistorted) wave when it squares up. 
S.-M. Choi and J. W. Lynn

NIST Center for Neutron Research

National Institute of Standards and Technology

Gaithersburg, MD 20899-8562 and

University of Maryland

College Park, MD 20742
D. Lopez, P. L. Gammel, and

C. M. Varma

Bell Laboratories

Lucent Technologies

Murray Hill, NJ 07974
P. C. Canfield and S. L. Bud'ko

Ames Laboratory and Department of Physics and Astronomy lowa State University

Ames, IA 50011

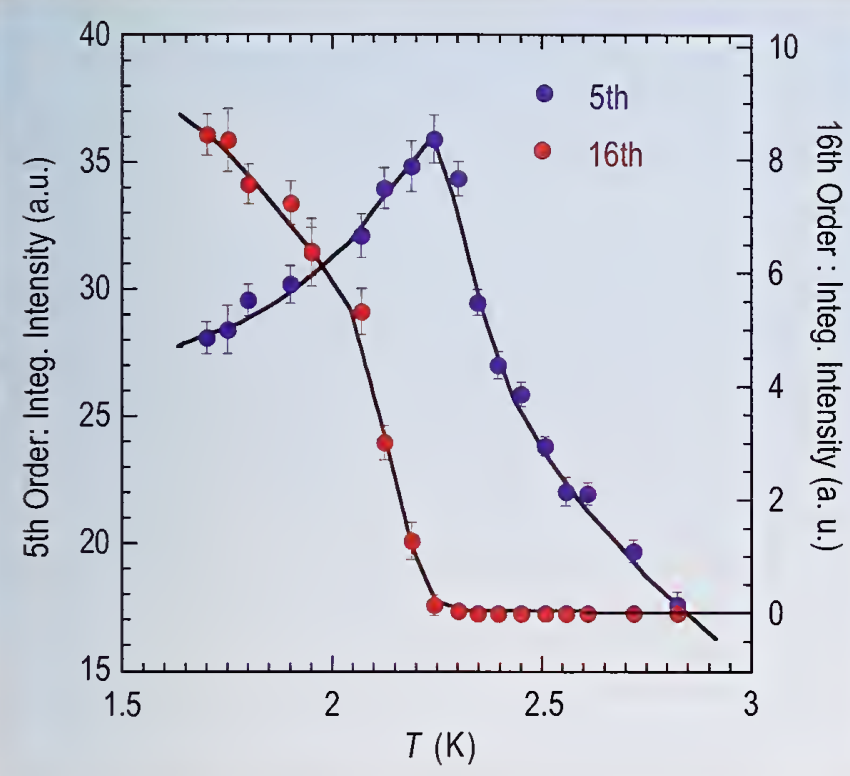

FIGURE 2. Integrated intensities of the odd (blue circles) and even (red circles) order harmonics as a function of $T$.

There is a substantial thermal hysteresis associated with the weak ferromagnetic transition, suggesting that this transition is first order in nature.

The present neutron results demonstrate that a net magnetization develops in $\mathrm{ErNi}_{2} \mathrm{~B}_{2} \mathrm{C}$ in the magnetically ordered state at low temperatures, making this the first such "ferromagnetic-superconductor" since $\mathrm{HoMo}_{6} \mathrm{~S}_{8}, \mathrm{HoMo}_{6} \mathrm{Se}_{8}$, and $\mathrm{ErRh}_{4} \mathrm{~B}_{4}$. For $\mathrm{ErNi}_{2} \mathrm{~B}_{2} \mathrm{C}$ the net magnetization is much smaller than for these earlier systems, which allows coexistence with superconductivity over an extended $T$ range. This presents the intriguing possibility that in an applied field vortices will form spontaneously when this net atomic magnetization is present. The small angle scattering data of the vortex structure (Fig. 3 top) show that the lattice has the expected spacing at lower applied fields. At higher fields, as the field begins to penetrate, vortices spontaneously form in addition to those expected from the applied field alone, increasing the vortex density and shifting the vortex peak. The $T$ dependence of this shift (Fig. 3 bottom) makes it clear that this behavior is directly related to onset of the net magnetization in the system. It is also interesting to note that the vortex pinning is enhanced in both magnetic phases, which may prove useful in high current applications of superconductors.
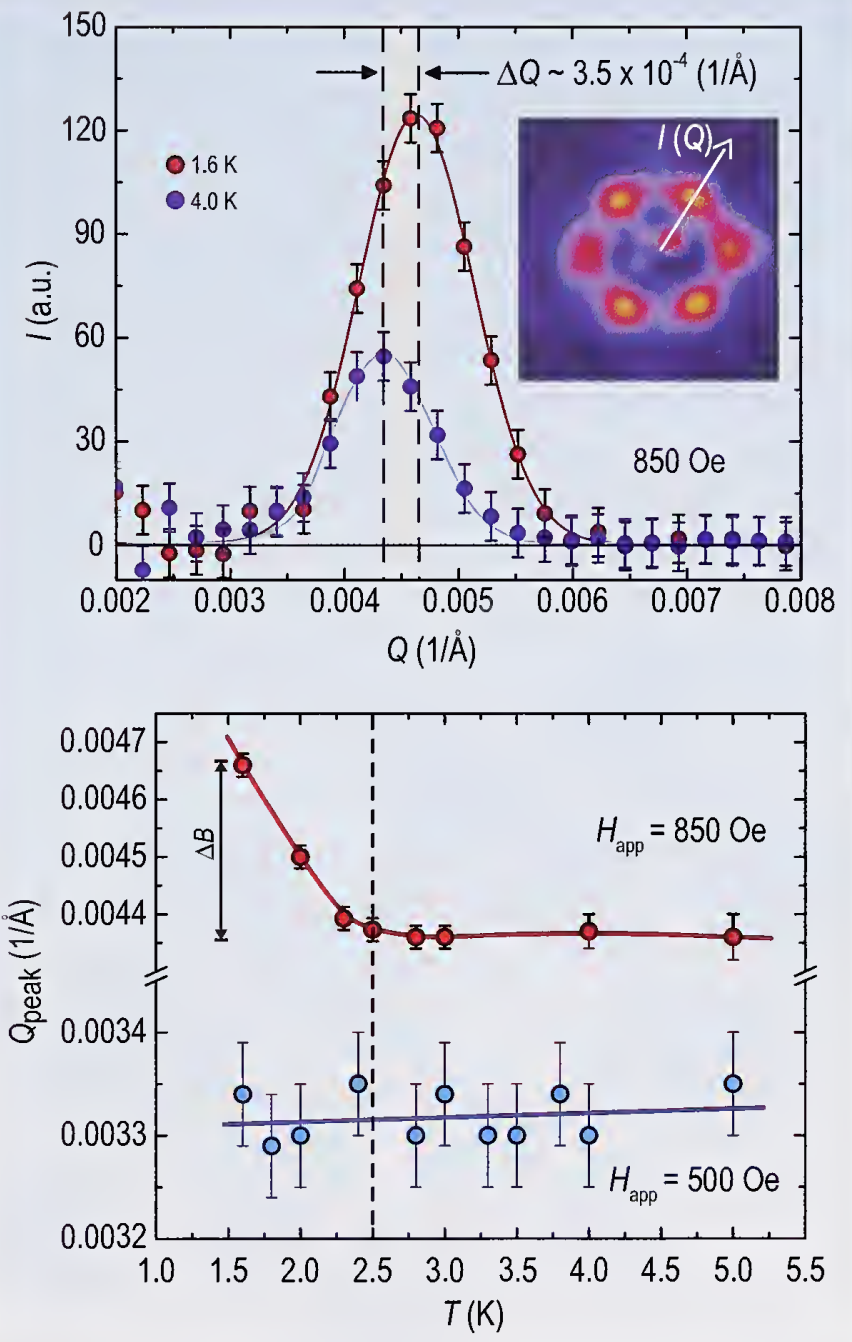

FIGURE 3. Intensity of the vortex scattering vs. wave vector $Q$ at $850 \mathrm{Oe}$ above and below the weak ferromagnetic transition. The shift in the peak position demonstrates that additional vortices spontaneously form at low temperatures. The temperature dependence (bottom) shows that this spontaneous vortex formation is directly related to the weak ferromagnetic transition.

\section{References}

[1] J. W. Lynn and S. Skanthakumar, Handbook on the Physics and Chemistry of Rare Earths, Chap. 199, Vol. 31, ed. by K. A. Gschneidner, Jr., L. Eyring, and M. B. Maple, North Holland, Amsterdam (2001) p. 313.

[2] J. W. Lynn, S. Skanthakumar, Q. Huang, S. K. Sinha, Z. Hossain, L. C. Gupta, R. Nagarajan, and C. Godart, Phys. Rev. B55, 6584 (1997).

[3] S.-M. Choi, J. W. Lynn, D. Lopez, P. L. Gammel, P. C. Canfield and S. L. Bud'ko, Phys. Rev. Lett. 87, 107001 (2001). 


\section{Structure of Local Spin Excitations in a Geometrically Frustrated Antiferromagnet}

or many crystalline magnetic materials, only a long
range ordered spin configuration can satisfy all near neighbor spin interactions. Such systems generally display a finite temperature transition to a broken symmetry phase with long-range magnetic order. However, on certain lattices with weak connectivity and a triangular motif, short-range interactions can be satisfied without long-range order [1].

To explore this possibility we examined magnetic order and fluctuation in $\mathrm{ZnCr}_{2} \mathrm{O}_{4}$. The B-site of this spinel lattice is solely occupied by spin- $3 / 2 \mathrm{Cr}^{3+}$, and this leads to a magnet with dominant nearest neighbor interactions on the lattice of corner-sharing tetrahedra shown in Fig. 1.

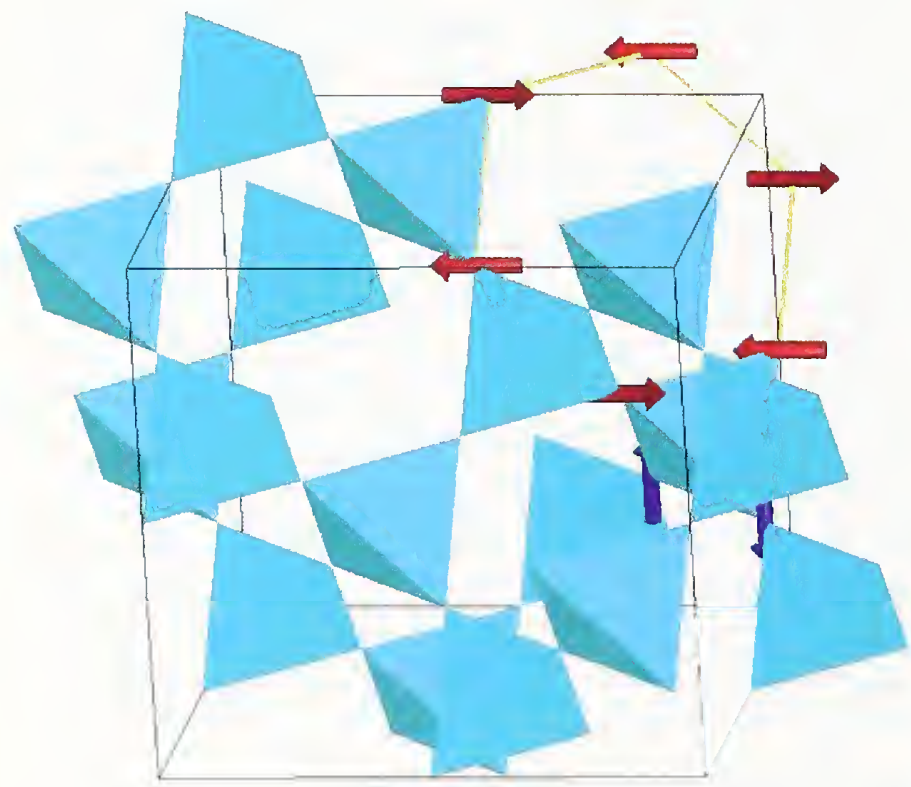

FIGURE 1. Lattice of corner-sharing tetrahedral formed by the octahedrally coordinated $\mathrm{B}$ sites of the spinel $\mathrm{AB}_{2} \mathrm{O}_{4}$. Black lines represent a structural unit cell containing eight tetrahedra. Vertices are populated by antiferromagnetically interacting spin-3/2 degrees of freedom in $\mathrm{ZnCr}_{2} \mathrm{O}_{4}$. Yellow line and six red spins represent a hexagonal loop of antiferromagnetically aligned spins.

Exchange interactions in this structure are satisfied at the mean field classical level when every tetrahedron carries zero net magnetization. Not only are there many ways that a tetrahedron can have no moment, but there are also many ways to organize such non-magnetic tetrahedra on the corner-sharing lattice. The result is a "geometrically frustrated" spin system with many ways to satisfy interactions without long-range order.
Analysis of specific spin models on the B-site spinel (or pyrochlore) lattice indicates that spin-1/2 and spin- $\infty$ models have short-range order down to $T=0$ [2], while long-range order is induced by quantum fluctuations for an unknown intermediate range of spin values. Experiments indicate that spin- $3 / 2 \mathrm{ZnCr}_{2} \mathrm{O}_{4}$ is "close" to the quantum critical point that separates the low spin quantum-disordered phase from the intermediate spin long-range ordered phase. Specifically, the relaxation rate for magnetic excitations, $\Gamma$, follows a power-law that extrapolates to zero as $T$ approaches 0 , indicating quantum criticality [3]. This state of affairs, however, does not persist to the lowest temperatures. Instead, at $T_{\mathrm{N}}=12.5 \mathrm{~K}$ a first order structural transition from the cubic cooperative paramagnet to tetragonal Néel order intervenes.

Figure 2 shows that a gapless continuum of magnetic scattering above $T_{\mathrm{N}}$ is pushed into a local spin resonance at $\hbar \omega \approx 4.5 \mathrm{meV} \approx J$ with remarkably little dispersion throughout the Brillouin zone. The result is analogous to the spinPeierls transition of the uniform spin- $1 / 2$ chain, where quantum critical fluctuations are pushed into a finite energy singlet-triplet transition through structural dimerization. Our

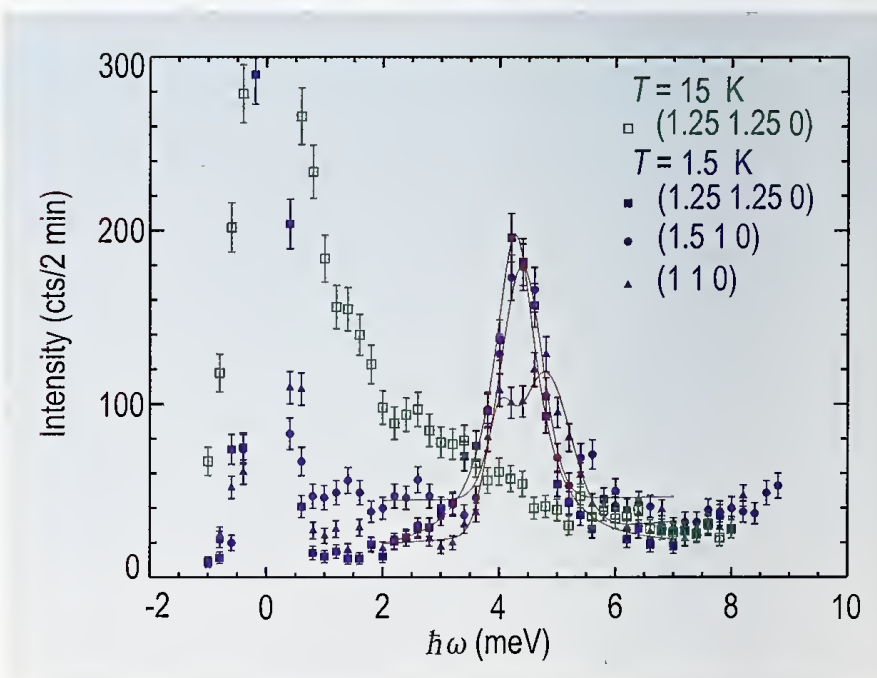

FIGURE 2. Constant- $Q$ scans above and below $T_{\mathrm{N}}=12.5 \mathrm{~K}$. In the cooperative paramagnetic phase above $T_{\mathrm{N}}$ magnetic excitations form a continuum. Below $T_{\mathrm{N}}$ the spectrum changes dramatically with a nearly dispersionless excitation near $4.5 \mathrm{meV}$ appearing abruptly. The red curves are to guide the eye. 
S.-H. Lee

NIST Center for Neutron Research

National Institute of Standards and Technology

Gaithersburg, MD 20899-8562 and

University of Maryland

College Park, MD 20742
C. Broholm and G. Gasparovic

Department of Physics and Astronomy

The Johns Hopkins University

Baltimore, MD 21218

T. H. Kim

Francis Bitter Magnet Laboratory

Massachusetts Institute of Technology

Cambridge, MA 02139 recent synchrotron $\mathrm{x}$-ray and neutron powder experiments indicate that deformation of tetrahedra does indeed occur for $\mathrm{ZnCr}_{2} \mathrm{O}_{4}$. However, the structural changes push the system to long range order rather than to quantum disorder, as indi-

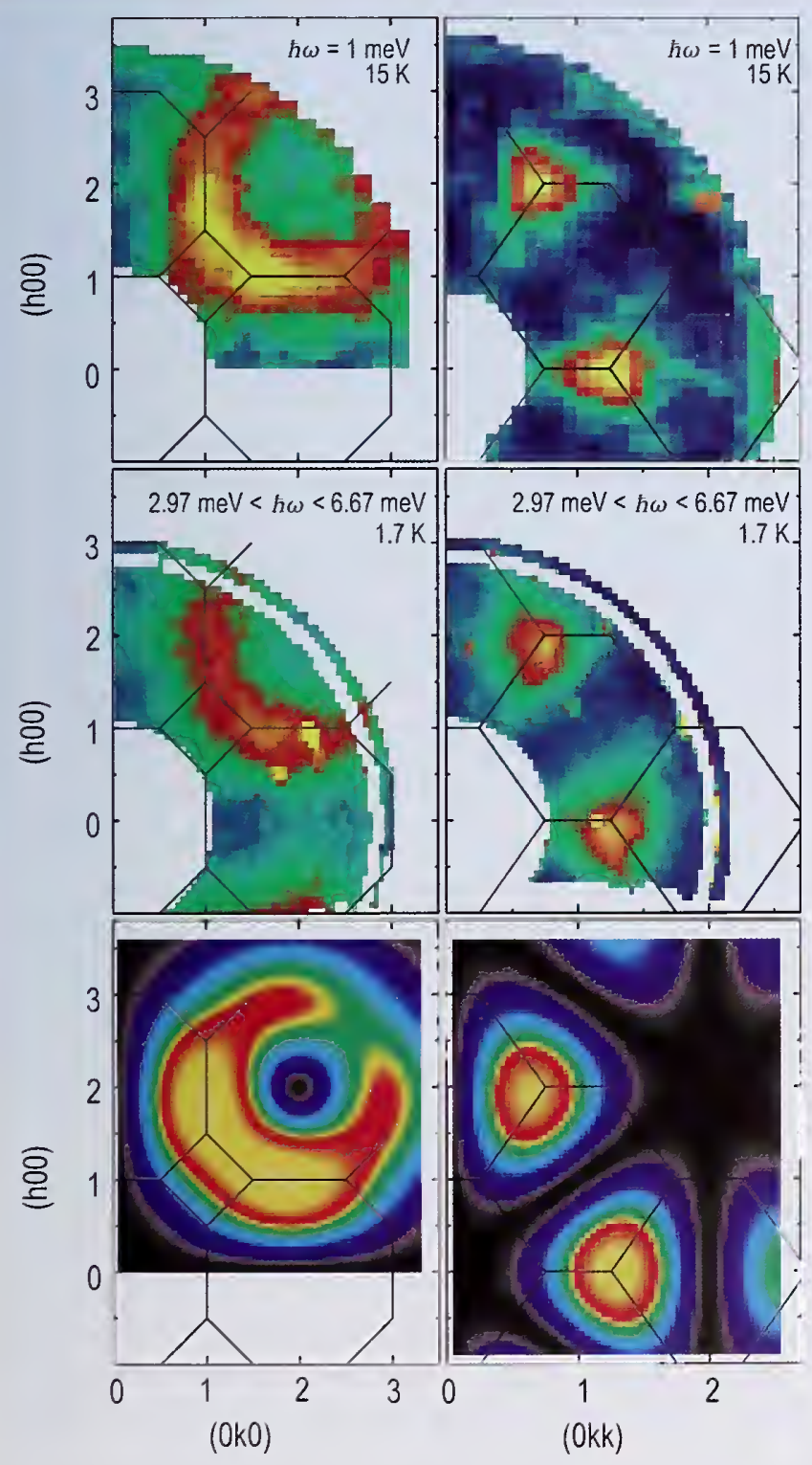

FIGURE 3. Color images of inelastic neutron scattering intensities in the (hkO) and (hkk) symmetry planes. From top to bottom are shown data from below and above $T_{N}$, and the structure factor for the hexagon mode proposed by 0 . Tchernyshyov. $T=1.7 \mathrm{~K}$ data were taken integrating over the energy range $\hbar \omega$ from $3.0 \mathrm{meV}$ to $6.7 \mathrm{meV}$. Data in the paramagnetic phase $(T=15 \mathrm{~K})$ were taken at $\hbar \omega=1 \mathrm{meV}$. cated by the magnetic Bragg peaks and spin wave excitations.

Our single crystal experiment [4] also enabled unique insight into the local structure of spin-fluctuations in geometrically frustrated systems. Figure 3 shows the $Q$-dependence of low energy magnetic scattering in two high symmetry planes above and below $T_{\mathrm{N}}$. While the spectrum for spin fluctuations changes dramatically at the first order phase transition, the structure factor clearly does not. Also shown in the figure is the structure factor for six spins of $<111>$ type kagomé hexagons precessing with $\pi$ phase shift between neighbors. The proposal by O. Tchernyshyov et al., [5] that these are the dominant low energy spin fluctuations for spins on the B-site spinel lattice is clearly borne out by the data.

The present data for $\mathrm{ZnCr}_{2} \mathrm{O}_{4}$ show that geometrically frustrated lattices have composite low energy degrees of freedom analogous to rigid unit modes in certain open framework lattice structures. To better understand the unusual type of phase transition that occurs in this system, it must be determined what defines the $4.5 \mathrm{meV}$ energy scale for hexagon excitation in the ordered phase. Do quantum fluctuations play a significant role or does the broken symmetry between exchange interactions within tetrahedra induce the resonance? The answer to this question is now being pursued through an accurate determination of the complex low temperature lattice and magnetic structure.

\section{References}

[1] P. W. Anderson et al., Philos. Mag. 25, 1 (1972); J. Villain, Z. Phys. B 33, 31 (1979).

[2] R. Moessner et al., Phys. Rev. Lett. 80, 2929 (1998); B. Canals et al., Phys. Rev. Lett. 80, 2933 (1998)

[3] S.-H. Lee, C. Broholm, T.H. Kim, W. Ratcliff II, and S.-W. Cheong, Phys. Rev. Lett. 84, 3718 (2000).

[4] S.-H. Lee et al., unpublished (2002).

[5] O. Tchernyshyov et al., unpublished (2002). 


\section{Pressure-Induced Phase Transition of $C_{12} E_{5}$ Micelles}

$\mathbf{N}$ onionic surfactants form a variety of microstructures in water, ranging from simple micelles at low surfactant concentrations to complex mesophases, such as hexagonal or lamellar phases at high concentrations. The effect of pressure on the structure of micelles and microemulsions has not been extensively studied. Small angle neutron scattering (SANS) is particularly well suited for such measurements because the range of length scales probed includes both the particle size and the interparticle spacing.

Pressure effects observed in SANS measurements of surfactant microstructure are typically interpreted in terms of geometric packing arguments that focus on the compressible hydrophobic tails of the surfactants. In this context, increasing pressure has the single effect of decreasing the surfactant tail volume, thereby increasing the curvature of the oil-water interface. Conversely, increasing temperature dehydrates the nonionic headgroups, decreasing the headgroup area and decreasing the film curvature. Thus, temperature and pressure can be viewed as thermodynamic variables with inherently different mechanisms and opposite effects for controlling microstructure in nonionic surfactant solutions.

Here we report the results of high-pressure SANS experiments at $20^{\circ} \mathrm{C}$ and pressures up to $\approx 300 \mathrm{MPa}$ on a solution of pentaethylene glycol mono-n-dodecyl ether $\left(\mathrm{C}_{12} \mathrm{E}_{5}\right)$ in $\mathrm{D}_{2} \mathrm{O}$ having a mass fraction of $1 \%$. The phase diagram for this system [1] at ambient pressure is shown in Fig. 1. At this temperature and surfactant concentration, a single-phase micellar solution ( $\mathrm{L}_{1}$ phase) forms at ambient pressure, well below the lower critical solution temperature (LCST) for liquid-liquid equilibrium and far removed from the $\mathrm{H}_{1}$ hexagonal phase at much higher $\mathrm{C}_{12} \mathrm{E}_{5}$ concentrations. The microstructure of the $\mathrm{L}_{1}$-phase at ambient pressure is known to be a network of branched semi-flexible, cylindrical micelles with the branch points comprised of three-armed junctions.

SANS spectra were measured using the NIST highpressure cell and neutrons of wavelength $\lambda=6 \AA$, covering a $q$-range of $0.012 \AA^{-1}<q<0.22 \AA^{-1}$. The scattering curves obtained at $3.4 \mathrm{MPa}, 241 \mathrm{MPa}$, and $255 \mathrm{MPa}$ are shown in Fig. 2. The curves at $3.4 \mathrm{Mpa}$ and $24 \mathrm{l} \mathrm{MPa}$ are virtually identical, indicating no significant change in microstructure with increasing pressure up to $241 \mathrm{MPa}$. Fitting these curves using a form factor for cylindrical micelles gives a radius of $(21.0 \pm 0.2) \AA$ and a length greater than $600 \AA$, independent of pressure. However, a small increase in pressure from $241 \mathrm{MPa}$ to $255 \mathrm{MPa}$ leads to the appearance of a peak in the scattering intensity at $q \approx 0.130 \AA^{-1}$, indicative of a locally ordered system. A similar transition has been reported in high-pressure SANS studies of tetradecyldimethylaminoxide (TDMAO) micelles in $\mathrm{D}_{2} \mathrm{O}$ at pressures up to $300 \mathrm{MPa}$, but the high-pressure microstructure was never determined [2].

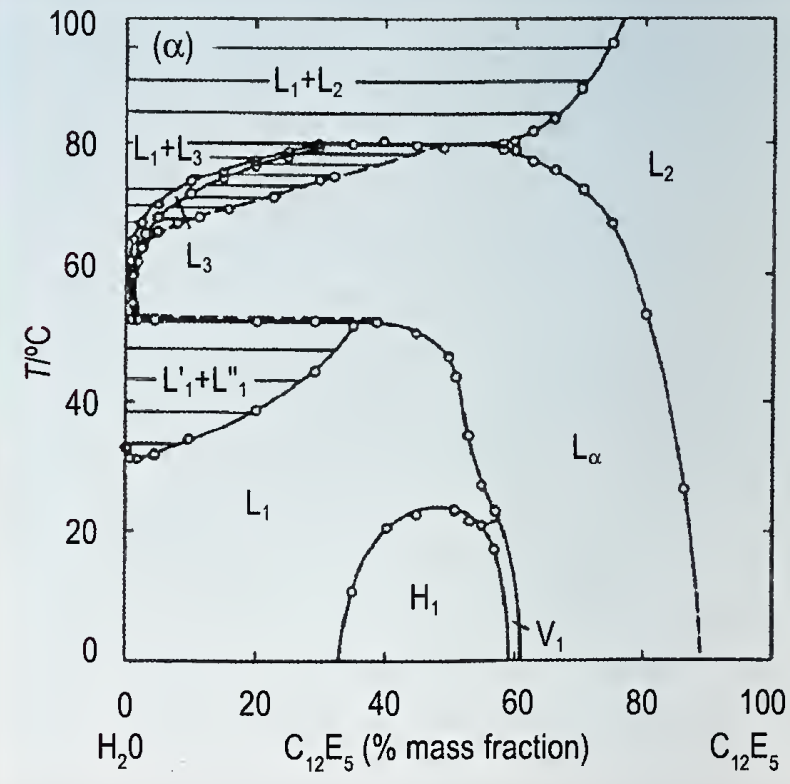

FIGURE 1. Temperature-composition phase diagram for $C_{12} E_{5} /$ water solutions at ambient pressures [1].

Shown in Fig. 2 is the scattering curve for the $\mathrm{H}_{1}$ hexagonal phase at $49 \% \mathrm{C}_{12} \mathrm{E}_{5}$ mass fraction at $20{ }^{\circ} \mathrm{C}$, and ambient pressure. The peak at $q \approx 0.120 \AA^{-1}$, arising from the hexagonal lattice of cylindrical micelles, is similar to the peak for the $1 \% \mathrm{C}_{12} \mathrm{E}_{5}$ mass fraction solution at $255 \mathrm{MPa}$, suggesting that this new high-pressure phase may resemble a slightly compressed state of the $\mathrm{H}_{1}$ hexagonal phase at ambient pressure. The formation of a lamellar phase from cylindrical micelles is unlikely, since this corresponds to increasing the hydrophobic core volume-to-surface-area ratio per surfactant molecule, or equivalently, decreasing the spontaneous curvature of the surfactant film. The application 


\section{S. R. Kline}

NIST Center for Neutron Research

National Institute of Standards and Technology

Gaithersburg, MD 20899-8562

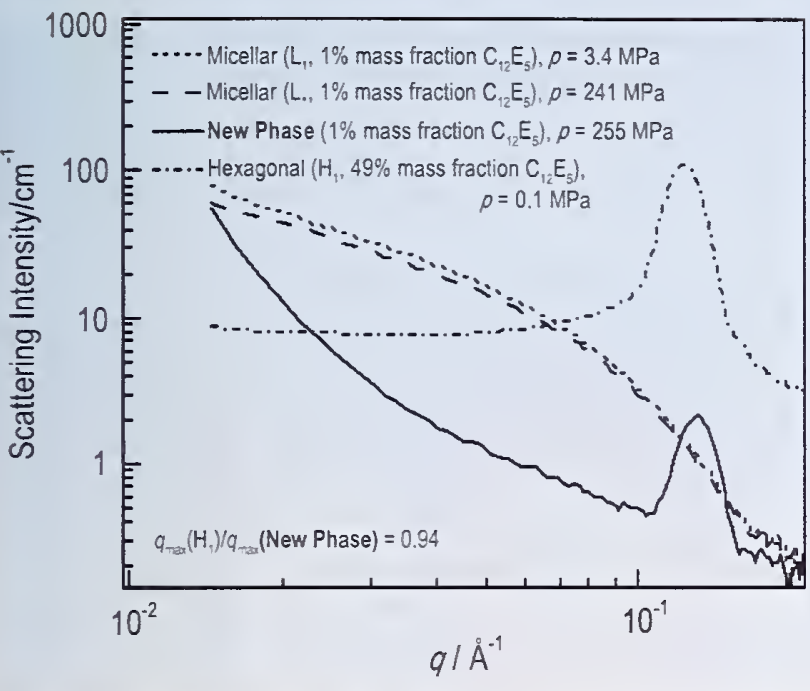

FIGURE 2. Measured SANS spectra at $20{ }^{\circ} \mathrm{C}$ for $1 \%$ mass fraction $C_{12} E_{5}$ in $D_{2} \mathrm{O}$ as a function of pressure and $49 \%$ mass fraction $C_{12} E_{5}$ in $D_{2} O$ at ambient pressure.

of pressure would have the opposite effect. We conclude, therefore, that the observed change in microstructure corresponds to a pressure-induced $\mathrm{L}_{1}-\mathrm{H}_{1}$ phase transition from a network of branched semi-flexible, cylindrical micelles to hexagonally ordered bundles of cylindrical micelles.

To further understand the SANS results, we have measured the temperature dependence of the $\mathrm{L}_{1}-\mathrm{H}_{1}$ transition pressure and find that the $p-T$ curve follows the $p-T$ freezing curves for liquid $n$-alkanes of comparable hydrocarbon chain length. $N$-decane solidifies at a pressure of $\approx 250 \mathrm{MPa}$ at $20{ }^{\circ} \mathrm{C}$. which is close to the pressure for the observed $\mathrm{L}_{1}-\mathrm{H}_{1}$ phase transition. We propose that the $\mathrm{C}_{12} \mathrm{E}_{5}$ micelle hydrophobic core, equivalent to $n$-decane, does solidify at these conditions, such that the micelles lose flexibility, and hence conformational entropy. An analysis of the geometric packing constraints for three-arm junctions coexisting with cylinders shows that when the surfactant tail volume decreases with increasing pressure, the fraction of surfactant forming junction points also decreases. This indicates that the formation of three-arm junctions becomes increasingly unfavorable at higher pressures due to the compression of the $\mathrm{C}_{12} \mathrm{E}_{5}$ micelle hydrophobic core. Our calculations predict that no junctions should be present at $P>275 \mathrm{MPa}$ at $20^{\circ} \mathrm{C}$, which is in good agreement with our observation of a structural transition between $241 \mathrm{MPa}$ and $255 \mathrm{MPa}$ at this temperature. Consequently, the network of branched threadlike micelles becomes globally unstable. The formation of hexagonally ordered bundles of cylindrical micelles follows as the attractive van der Waals forces between the micelles are not offset by entropic repulsive undulation interactions that are not present in the now-solidified hydrophobic core.

The practical significance of these results is to show that pressure allows access to regions of the $\mathrm{C}_{12} \mathrm{E}_{5}$ /water phase diagram that are virtually inaccessible to temperature. Thus, the use of pressure may offer unique approaches for directing and stabilizing certain surfactant microstructures that, in turn. could prove useful for creating novel soft materials.

\section{References}

[1] R. Strey, R. Schomäcker, D. Roux, F. Nallet, U. Olsson, J. Chem. Soc. Faraday Trans. 86, 2253 (1990).

[2] N. Gorski, J. Kalus, D. Schwahn, Langmuir 15, 8080 (1999). 


\section{Probing the Structure of Aerosol Nanodroplets}

$\mathbf{N}$ anodroplet aerosols form readily in the supersonic expansions that occur, for example, in turbomachinery, jet exhausts, and volcanic eruptions. Thus, understanding particle formation and growth when cooling rates approach $10^{6} \mathrm{~K} / \mathrm{s}$ is of broad scientific interest.

From a fundamental point of view, particles with radii $<10 \mathrm{~nm}$ are important because they lie in the critical transition zone between large molecular clusters and bulk material. In multicomponent droplets, there is also strong theoretical evidence for surface enrichment, i.e., that the surface and interior compositions can differ significantly. Surface enrichment is important because it affects nucleation, growth and evaporation kinetics, and the heterogeneous chemistry of aerosol droplets. A major goal of our work is to use small angle neutron scattering to find direct evidence for surface enrichment in nanodroplets.

We produce nanodroplets with radii between $5 \mathrm{~nm}$ and $12 \mathrm{~nm}$ and number densities in the range of $10^{11} \mathrm{~cm}^{-3}$ to $10^{12} \mathrm{~cm}^{-3}$ by rapidly expanding a dilute vapor mixture of $\mathrm{D}_{2} \mathrm{O}$ (or other condensible) and $\mathrm{N}_{2}$ in a supersonic nozzle apparatus (Fig. 1) [1]. The volume fraction of droplets is always

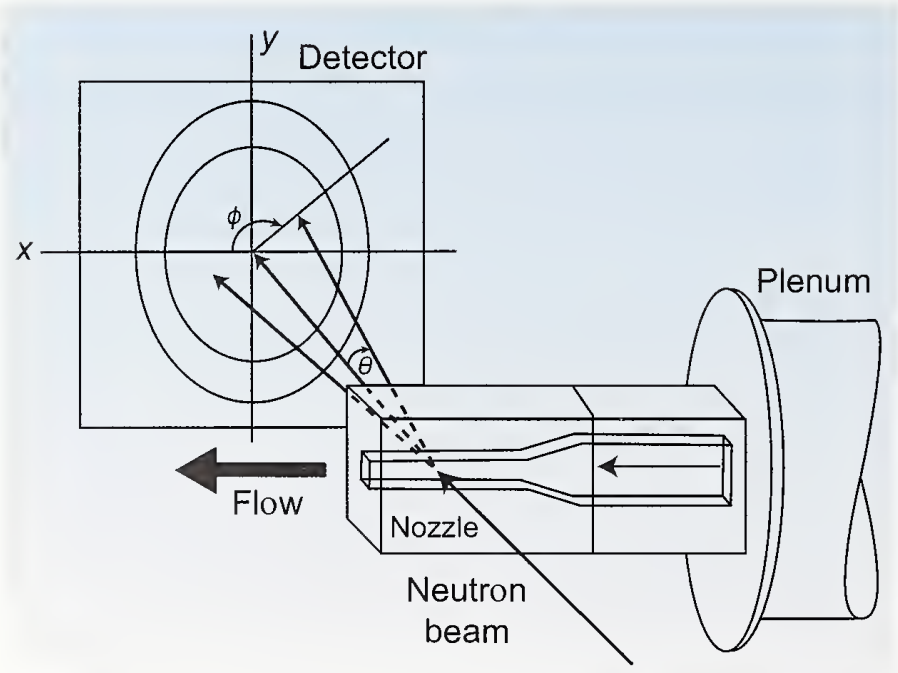

FIGURE 1. The nozzle is placed in the sample chamber at right angles to the neutron beam. less than $10^{-5}$ and, thus, the scattering signals are close to the instrument background. Furthermore, the high velocity of the droplets $(400 \mathrm{~m} / \mathrm{s}$ to $500 \mathrm{~m} / \mathrm{s})$ is comparable to the speed of the neutrons. This leads to a Doppler shift in the momentum of the scattered neutrons [2,3], and the 2-D scattering
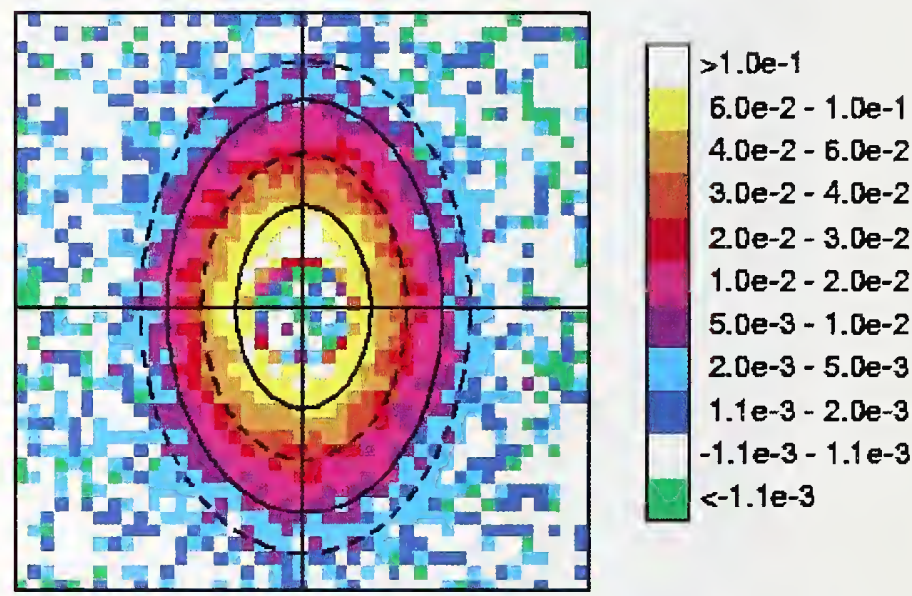

FIGURE 2. The observed 2-D scattering pattern for an aerosol with an average particle velocity $v_{p}=435 \mathrm{~m} / \mathrm{s}$ measured using a neutron wavelength of $\lambda=1.0 \mathrm{~nm}\left(v_{n}=400 \mathrm{~m} / \mathrm{s}\right)$. The contour levels correspond to absolute intensities of $0.08,0.03,0.008$ and $0.003 \mathrm{~cm}^{-1}$, respectively.

patterns are anisotropic (Fig. 2) even though spherical droplets at rest scatter isotropically. This Doppler shift can be used to directly measure the velocity of the particles, and, in a test case, this velocity was within $2 \%$ of the average velocity derived from the pressure trace measurements [2].

Rather than simply examine nanodroplets for which segregation of the components is severe, we use different materials to observe the transition between well-mixed and fully segregated droplets. Thus, our experiments include binary mixtures of $\mathrm{H}_{2} \mathrm{O}, \mathrm{D}_{2} \mathrm{O}$, ethanol, and n-butanol, or its fully deuterated analog d-butanol. Almost degenerate mixtures, such as $\mathrm{D}_{2} \mathrm{O}-\mathrm{H}_{2} \mathrm{O}$, appear to form well-mixed droplets [4]. In contrast, binary nanodroplets containing $\mathrm{H}_{2} \mathrm{O}$ (or $\mathrm{D}_{2} \mathrm{O}$ ) and a low molar concentration of d-butanol (or h-butanol) should consist of a water-rich core and an 
B. E. Wyslouzil, C. H. Heath,

U. M. Dieregsweiler,

and K. A. Streletzky

Department of Chemical Engineering

Worcester Polytechnic Institute

Worcester, MA 01609
R. Strey, J. Wölk

Institut für Physikalische Chemie

Universität zu Köln

D-50939 Cologne, Germany

G. Wilemski

Physics Department

University of Missouri - Rolla

Rolla, MO 65409 alcohol-rich shell. Our recent SANS experiments with $\mathrm{H}_{2} \mathrm{O}$ - d-butanol nanodroplets clearly demonstrate for the first time the existence of the alcohol-rich shell. As illustrated in Fig. 3, for these droplets the scattered intensity falls off as $q^{-2}$ in the high $q$ region, as is characteristic for scattering by a shell. Furthermore, the feature in the region $0.4 \mathrm{~nm}^{-1}<q<0.5 \mathrm{~nm}^{-1}$ cannot be reproduced by a wellmixed droplet model that matches the low $q$ data. Also shown in Fig. 3 is the scattering spectrum from the complementary $\mathrm{D}_{2} \mathrm{O}-\mathrm{h}$-butanol experiment. As expected, the scattered intensity is much higher, and the signal intensity decreases as $q^{-4}$ in the high $q$ region. In this case, the $\mathrm{D}_{2} \mathrm{O}$-rich core dominates the scattering.

In summary, SANS provides us with a powerful new way to study the properties of nanometer sized liquid droplets in the environment in which they form. To date, it is the only technique to directly probe the microstructure of aerosol nanodroplets. Combined with pressure trace measurements and modeling, SANS provides information critical to our understanding of droplet formation and growth in the nanometer size regime.

\section{References}

[1] B. E. Wyslouzil, J. L. Cheung, G. Wilemski, and R. Strey, Phys. Rev Lett. 79, 431 (1997).

[2] B. E. Wyslouzil, G. Wilemski, J. L. Cheung, R. Strey, and J. Barker, Phys. Rev. E 60, 4330 (1999).

[3] G. Wilemski, Phys. Rev. E 61, 557 (2000).

[4] C. H. Heath. K. A. Streletzky, J. Wolk, B. E. Wyslouzil, and R. Strey, in Nucleation and Atmospheric Aerosols, 2000, ed. by B. N. Hale and M. Kulmala, American Institute of Physics, New York (2000) p. 59.

\section{J. Barker}

NIST Center for Neutron Research

National Institute of Standards and Technology

Gaithersburg, MD 20899-8562

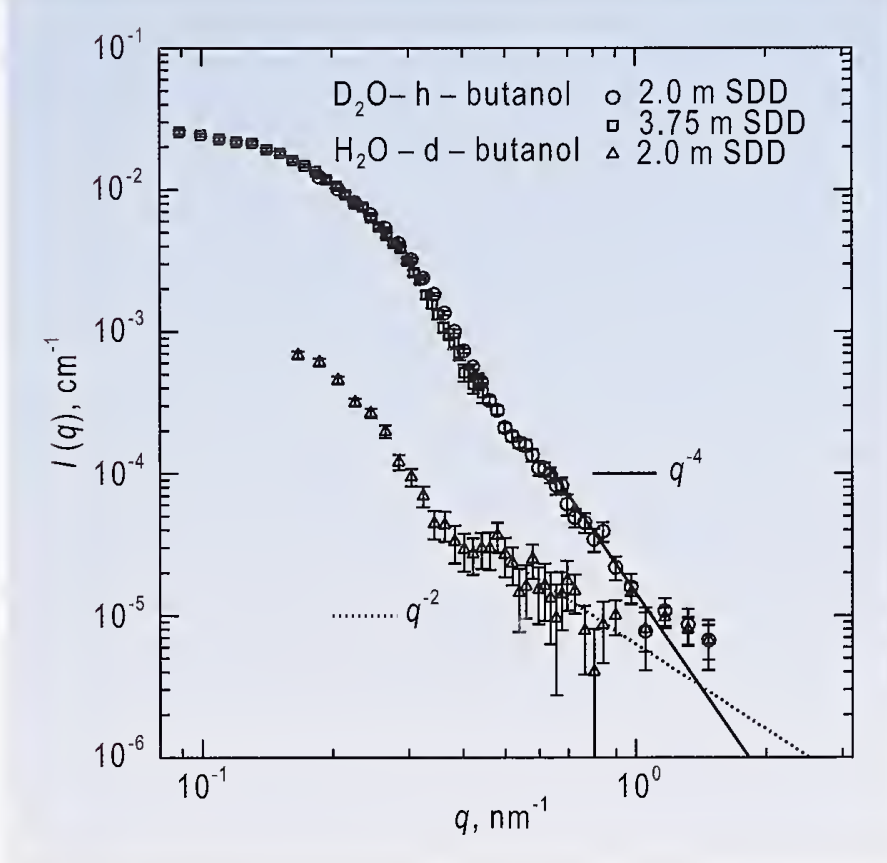

FIGURE 3. Scattering from a $D_{2} O-h$-butanol aerosol is compared to that from a $\mathrm{H}_{2} \mathrm{O}-\mathrm{d}$-butanol aerosol formed under identical conditions in the nozzle. Both aerosols contain $\approx 6 \%$ molecular fraction butanol. In the high $q$ region, the intensity falls off as $q^{-4}$ for the $D_{2} 0$-rich droplets, but only as $q^{-2}$ for the $\mathrm{H}_{2} \mathrm{O}$-rich droplets. Our preliminary modeling shows that the feature in the region $0.4 \mathrm{~nm}^{-1}<q<0.5 \mathrm{~nm}^{-1}$ of the $\mathrm{H}_{2} \mathrm{O}-\mathrm{d}$-butanol spectrum cannot be reproduced by a well mixed droplet model. SDD is the sample-to-detector distance. 
T he next generation of interlayer dielectric materials for microelectronics must have an ultra-low dielectric constant of less than 2.0 to meet the National Technology Roadmap for Semiconductors [1]. In addition, the material must have excellent thermal stability and mechanical properties $[2,3]$. One route is to introduce nanometer sized pores into a material with an initially low dielectric constant ( $k \approx 2$ to 3 ). The presence of the pores lowers the dielectric constant of the nanoporous film as the dielectric constant of air is 1.0. One class of materials that is receiving significant attention is based on poly(methylsilsesquioxane) (PMSSQ) which has a dielectric constant between 2.7 and 2.8 (lower than conventional $\mathrm{SiO}_{2}$ ) and good thermal stability up to $500{ }^{\circ} \mathrm{C}[4,5]$. The dielectric constant is a function of the level of porosity and detailed knowledge of the morphology is required for implementation in electronic packaging.

A schematic of the synthesis process is shown in Fig. 1. The materials are based on an inorganic matrix of PMSSQ containing a porogen (i.e., a labile pore generating material) based on a poly(methylmethacrylate) copolymer (coPMMA) as a template for creating the pores [6, 7]. A film is prepared by dissolving the PMSSQ matrix precursor material and the coPMMA porogen in a common solvent and spin casting onto a silicon substrate.

The film is then slowly ramped up in temperature to $450{ }^{\circ} \mathrm{C}$. During this temperature ramp, the matrix film fully cures by about $225^{\circ} \mathrm{C}$, resulting in microphase separation of the coPMMA polymer. Above about $350^{\circ} \mathrm{C}$ the coPMMA

PMMA copolymer

porogen material

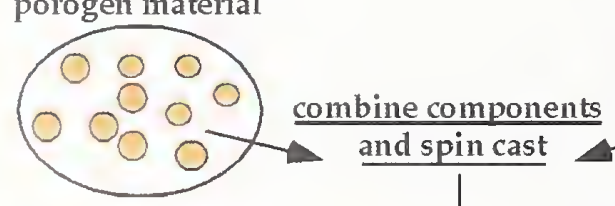

porogen material or pores

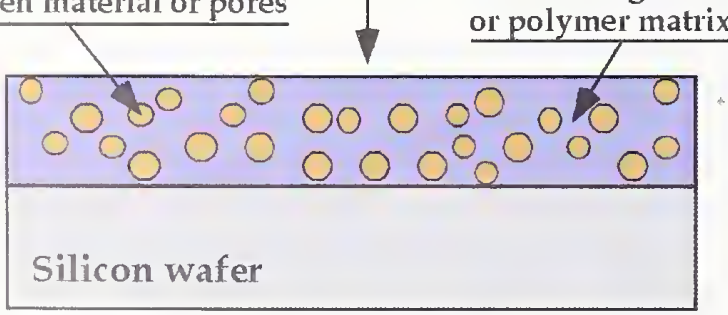

FIGURE 1. Schematic showing the generation of nanoporous PMSSQ films. polymer degrades leaving nanometer sized pores. Neutron scattering was used with a deuterated coPMMA polymer to understand the morphological development of the films during the cure. The use of the deuterated porogen provides contrast for neutron scattering in the hybrid (polymer containing) system that is not available with $\mathrm{x}$-ray scattering.

In situ studies of the curing process by small angle neutron scattering (SANS) were performed. To provide sufficient scattering signal, four or more samples on $\mathrm{Si}$ wafers were stacked to increase the scattering volume.

Figure 2 shows a set of SANS data from a PMSSQ thin film containing $20 \%$ molecular fraction of porogen at various cure temperatures. The scattered intensity varies as a function of curing temperature due to morphology changes occurring in the sample. For the as-spun materials there is a shoulder at high $q$ from which a radius of gyration $\left(R_{\mathrm{g}}\right)$ of $12 \AA$ was obtained by Guinier analysis. After heat treatment at $225^{\circ} \mathrm{C}$ the shoulder in $I(q)$ becomes more pronounced and shifts to about $q=0.06 \AA^{-1}$. The shift to smaller $q$ indicates a coarsening of the microphase separated porogen domain structure. A radius of gyration of $R_{\mathrm{g}}=23 \AA$ for the domains was obtained for the cured materials. The scattering curves do not change significantly between $225^{\circ} \mathrm{C}$ and $300^{\circ} \mathrm{C}$, indicating that the morphology remains fixed until the degradation of the polymer at $450{ }^{\circ} \mathrm{C}$. The dramatic

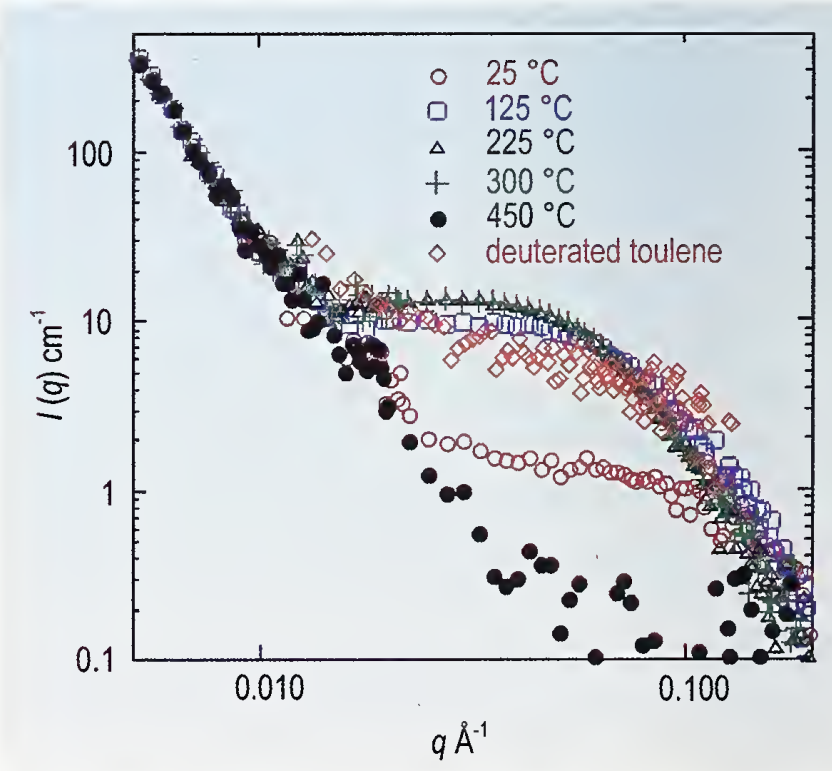

FIGURE 2. SANS data taken during cure of a nanoporous PMSSQ thin film. 
G. Y. Yang and R. M. Briber

Department of Materials and Nuclear Engineering

University of Maryland

College Park, MD 20742
E. Huang, H.-C. Kim,

W. Volksen, and R. D. Miller

IBM Almaden Research Center

San Jose, CA 95120
K. Shin

Department of Materials Science and Engineering State University of New York

Stony Brook, NY 11794 decrease in intensity of the SANS curve at $450{ }^{\circ} \mathrm{C}$ is due to a loss of neutron scattering length density contrast because of the degradation of the porogen and the formation of pores in the film.

To provide neutron contrast between the pores and the matrix, the fully cured sample was exposed to deuterated toluene. These data are also shown in Fig. 2. The scattering is largely recovered after exposure to deuterated toluene, indicating that the porous structure is maintained with no collapse after degradation of the polymer.

Figure 3 shows a set of neutron reflectivity profiles and scattering length density (SLD) profiles of a nanoporous PMSSQ thin film prepared with $20 \%$ molecular fraction of porogen and deposited on a silicon substrate. The film was exposed to deuterated toluene liquid and vapor to examine solvent swelling. The film thickness is observed to increase upon exposure to both liquid and vapor. A higher porosity is observed in a $50 \AA$ region next to the silicon surface. This is probably due to porogen surface segregation at the substrate interface and is in agreement with results from transmission electron microscopy.

\section{References}

[1] The National Technology Roadmap for Semiconductors: Technology Needs, SIA, Semiconductor Industry Association, 1997 Edition, p. 101.

[2] R. D. Miller, J. L. Hedrick, D. Y. Yoon, R. F. Cook, and J. P. Hummel, Mater. Res. Soc. Bulletin 22, 44 (1997).

[3] C. Jin, J. D. Luttmer, D. M. Smith, and T. A. Ramos, Mater. Res. Soc. Bulletin 22, 39 (1997).

[4] C. V. Nguyen, R. B. Beyers, C. J. Hawker, J. L. Hedrick, R. L. Jaffe, R. D. Miller, J. F. Remenar, H. W. Rhee, M. F. Toney, M. Trollsas, and D. Y. Yoon, Polymer Preprint 40 (1), 398 (1999).

[5] R. F. Cook, E. G. Liniger. D. P. Klaus, E. E. Simonyi, and S. A. Cohen, Mater. Res. Soc. Symp. Proc. 511, 33 (1998).

[6] C. V. Nguyen, K. R. Carter, C. J. Hawker, J. L. Hedrick, R. L. Jaffe, R.D. Miller, J. F. Remenar, H. W. Rhee, P. M. Rice, M. F. Toney, M. Trollsas, and D. Y. Yoon, Chem. Mater. 11, 3080 (1999).

[7] C. Nguyen, C. J. Hawker, R. D. Miller, E. Huang, and J. L. Hedrick, Macromolecules 33, 4281 (2000).
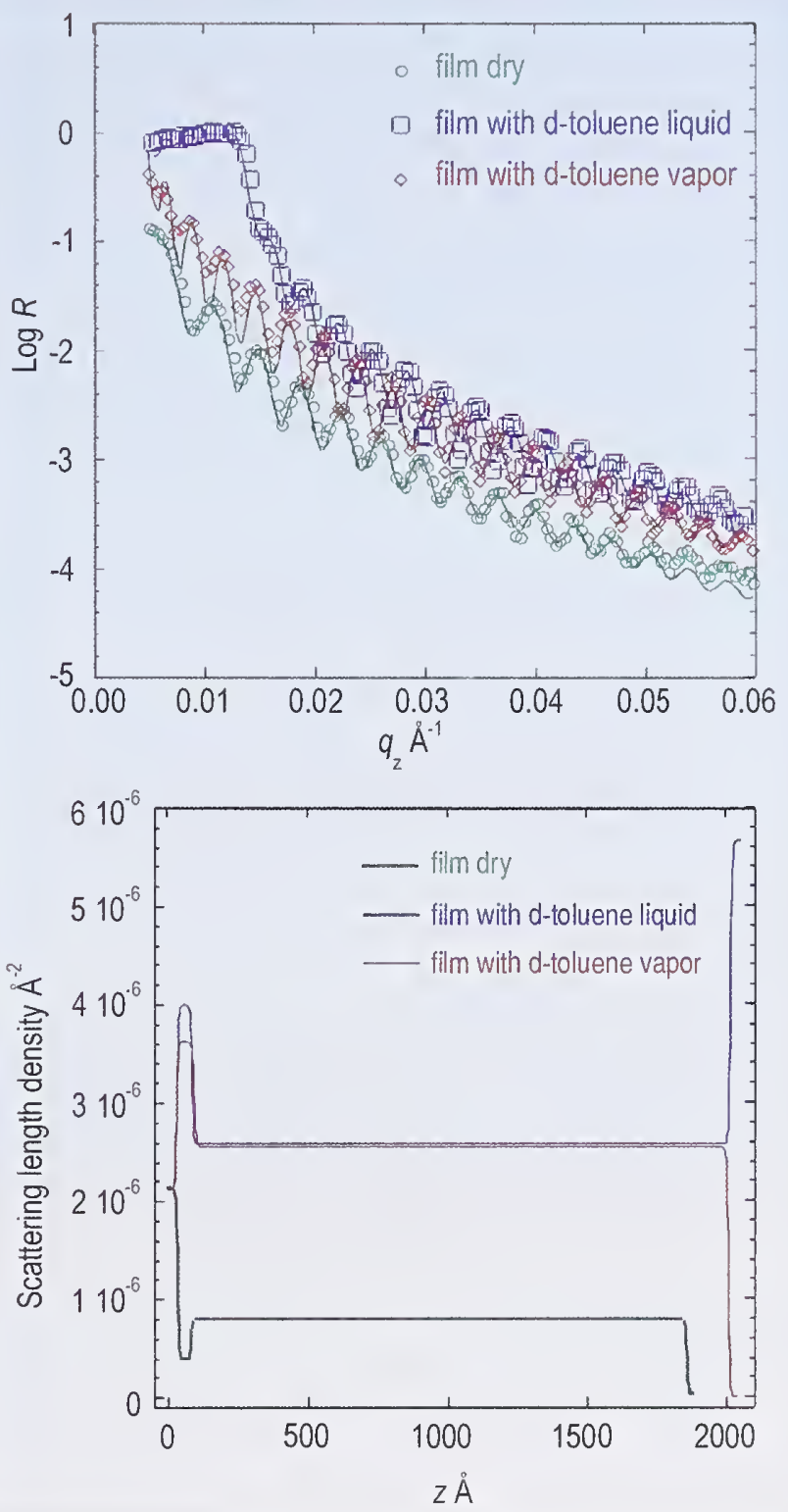

FIGURE 3. Above, neutron reflectivity data (symbols) and best fit (lines) calculated from the scattering length density profiles shown below for a nanoporous PMSSQ thin film under various conditions. 


\section{Neutron Reflectivity Studies of Surfactants at Electrode Surfaces}

$\mathrm{K}$ nowledge of the forces that control the assembly of surfactant molecules at the solid-liquid interface is vital for traditional fields such as detergency, flotation, oil recovery and tribology [1]. Thin organic films deposited at solid surfaces also find application in the fabrication of optoelectronic devices, sensors, biosensors, and chemically modified electrodes [2]. It has long been established that the assembly of surfactants at the solid-liquid interface depends on the charge at the solid surface [3,4]. For example, the spreading of vesicles into a phospholipid bilayer requires that the surface of the solid be negatively charged and hydrophilic.

However, the present understanding of the role played by charge on the interaction of a surfactant molecule with the electrified solid surface is far from being complete. Electrochemistry provides a unique opportunity to study the effect of the charge on the properties of amphiphilic and ionic surfactants at the charged solid-liquid interface [5]. When an organic film is deposited on a gold electrode, the charge density at the metal surface may be varied from about $30 \mu \mathrm{C} / \mathrm{cm}^{2}$ to about $40 \mu \mathrm{C} / \mathrm{cm}^{2}$. This magnitude of charge generates electric fields on the order of $10^{10} \mathrm{~V} / \mathrm{m}$. Such a field interacts with polar molecules in the membrane. By changing the sign of the charge one can use attractive or repulsive forces. In this manner, by turning a knob on a control instrument one can force phase transitions in the film of organic molecules or force surfactants to desorb or readsorb on the surface.

We have recently employed electrochemical techniques, atomic force microscopy, and neutron reflectivity to study the field driven transformations of thin films formed by a model anionic surfactant, sodium dodecyl sulfate (SDS), at the surface of a gold electrode [6]. Figure 1 shows how the surface concentration of SDS at the Au electrode surface changes with the electrode potential. A convenient way to interpret these data is to look at the electrode potential as an operational variable that can be easily adjusted using a control instrument.

Figure 1 shows that the character of SDS adsorption is strongly influenced by the charge on the metal. At sufficiently negative potentials SDS molecules are totally desorbed from the electrode surface. At moderate negative

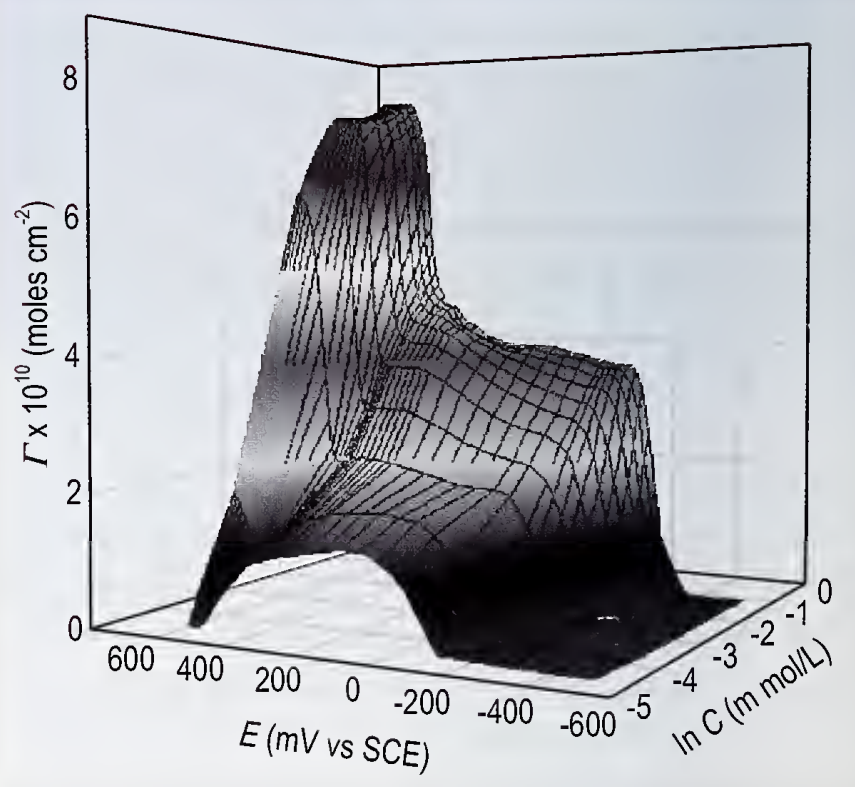

FIGURE 1. Three dimensional plots of the surface concentration of SDS as a function of electrode potential measured versus the calomel reference electrode (SCE) and the logarithm of the bulk SDS concentration.

charge densities SDS forms a film characterized by a limiting surface concentration $4.0 \times 10^{-10} \mathrm{~mol} \mathrm{~cm}^{-2}$. When the metal surface is positively charged the surface concentration of SDS increases to $8.1 \times 10^{-10} \mathrm{~mol} \mathrm{~cm}$.

Neutron reflectivity experiments carried out on the NG-7 reflectometer were employed to determine the structure of the film formed by SDS at different charge densities at the gold surface. Thin layers of chromium $(\approx 20 \AA)$ and gold $(\approx 80 \AA)$ were sputtered onto the crystal quartz substrate. After cleaning, the crystal was mounted on a specially constructed Teflon ${ }^{\circledR}$ cell [7]. The cell had ports for the counter (gold foil) and reference electrodes $(\mathrm{Ag} / \mathrm{AgCl}$, $E \approx-40 \mathrm{mV}$ versus SCE). $\mathrm{D}_{2} \mathrm{O}$ (99.9\% molecular fraction) was used as a solvent in reflectivity studies.

Figure 2 a shows the neutron reflectivity data determined for SDS adsorption at various electrode potentials, and Fig. $2 \mathrm{~b}$ shows the scattering length density profiles calculated from the reflectivity curves. The neutron reflectivity data are consistent with electrochemical measurements. They show that at very negative potentials the gold solution interface is free from hydrogenated species. When 
I. Burgess, V. Zamlynny, G. Szymanski, and J. Lipkowski

Department of Chemistry and Biochemistry

University of Guelph

Guelph, ONT N1G 2W1, Canada
J. Majewski and G. Smith

Los Alamos National Laboratory

Los Alamos, NM 87545
S. Satija and R. Ivkov

NIST Center for Neutron Research

National Institute of Standards and

Technology

Gaithersburg, MD 20899-8562
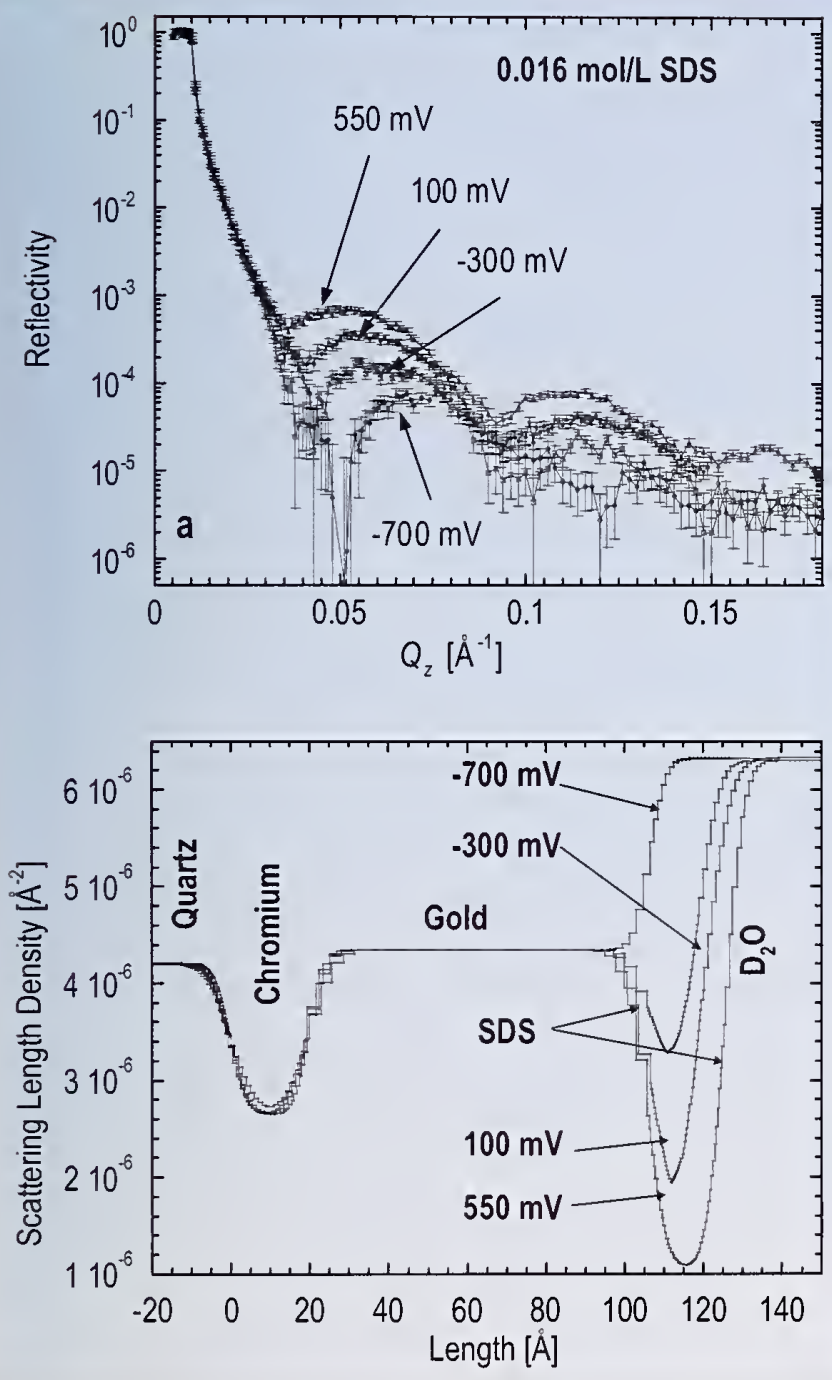

FIGURE 2. (a) Normalized neutron reflectivity curves for a Au/Cr-coated quartz substrate in $0.016 \mathrm{~mol} / \mathrm{L}$ SDS in $D_{2} O$. (b) Scattering length density profiles of the interface as determined from the fitting procedure.

the potential increases, the film of hydrogenated species appears at the electrode surface. The thickness of this film increases, and the scattering length density progressively decreases, with increasing potential. When combined with the results of electrochemical measurements and atomic force imaging, the neutron reflectivity data allow the determination of the structure of the film formed at different charge densities. At small or moderate negative charge densities SDS molecules form a hemimicellar film that consists of hemicylindrical stripes, as first observed by
Manne [4]. The packing of SDS molecules in a cross section of that hemicylinder is shown schematically in Fig. 3 (top). At positive charge densities the hemimicellar state is transformed into the interdigitated bilayer schematically shown in Fig. 3 (bottom).

The results of this study demonstrate the need for the use of neutron reflectometry to study adsorption and phase transitions in films of surfactants adsorbed at the solidsolution interface. Specifically, they show that when neutron reflectivity measurements are combined with electrochemical studies and atomic force microscopy, they provide unique opportunities to study different stages involved in the interaction of surfactants with solid surfaces.
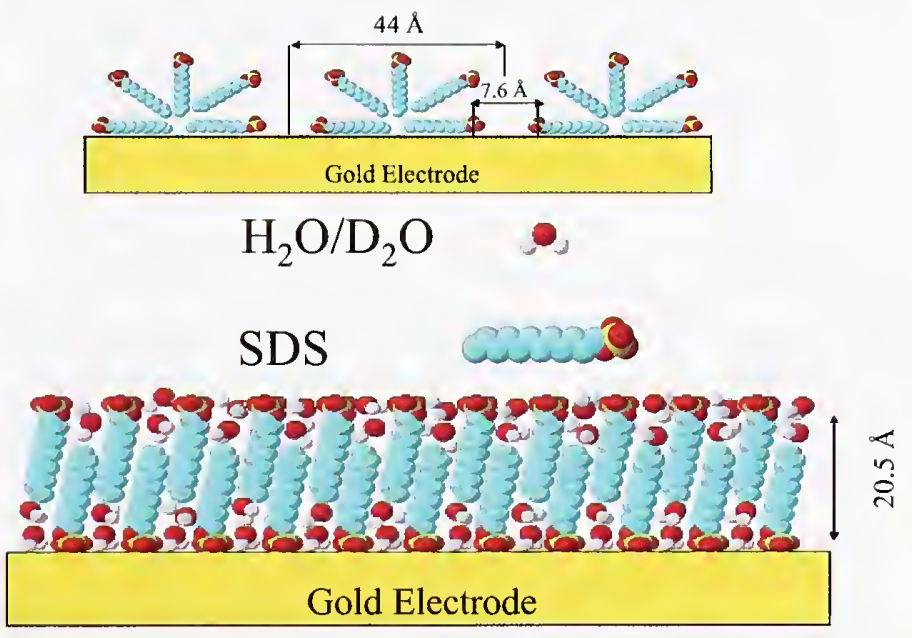

FIGURE 3. Models of SDS adsorption at the Au-solution interface: (top) cross section of hemicylindrical aggregates observed at moderately negative charge densities; (bottom) interdigitated bilayer observed at positive charge densities.

\section{References}

[1] A.W. Adamson, Physical Chemistry of Surfaces, $5^{\text {th }}$ ed., John Wiley \& Sons, New York (I990).

[2 E. Sackmann, Science 271, 43 (1996).

[3] P. Chandar, P. Somasundaran, N. J. Turro, J. Colloid Interface Sci. 117, 31 (1987)

[4] S. Manne, Progr. Colloid Polym. Sci. 103, 226 (1997).

[5] D. Bizzotto, J. Lipkowski, J. Electroanal. Chem. 409, 33 (1996).

[6] V. Zamlynny, I. Burgess, G. Szymanski, J. Lipkowski, J. Majewski. G. Smith, S. Satija, and R. Ivkov, Langmuir 16, 9861(2000).

[7] I. Burgess, V. Zamlynny, G. Szymanski, J. Lipkowski, J. Majewski. G. Smith, S. Satija, and R. Ivkov, Langmuir 17, 3355 (200I). 
urrently a great deal of attention is being focused on

spintronics, a new area of solid-state electronics. In spintronics not only the electric current but also its spin state is controlled. Spin valves and spin injectors are the first practical applications of spintronics. Further progress in developing new devices hinges critically on the availability of suitable materials. Such materials need to be "good" semiconductors, easy to integrate in typical integrated circuits, and their electronic properties should exhibit strong sensitivity to the carrier's spin, ferromagnetism being an especially desirable property.

EuS is one of the very few natural ferromagnetic (FM) semiconductors. Since it becomes FM at a low temperature $\left(T_{c}=16.6 \mathrm{~K}\right)$ it is an unlikely choice for applications.

However, studying the properties of heterostructures made on its base may give an important insight into fundamental processes taking place in all classes of materials under consideration.

GaMnAs is a man-made FM semiconductor. It is an example of a diluted magnetic semiconductor (DMS) in which a fraction of nonmagnetic cations (Ga) is substituted with magnetic ions (Mn). Such a material can readily be incorporated into modern GaAs-based semiconductor devices. Its $T_{\mathrm{c}}$ is still below room temperature, but this limitation may be lifted in other materials of this class [1].

Interlayer exchange coupling (IEC) in superlattices (SL), composed of ferromagnetic and nonmagnetic layers, is a crucial element of all spin-valve type devices that utilize the giant magnetoresistance effect. In metallic SLs currently being used, conduction electrons transfer the interlayer interactions through nonmagnetic spacers [2]. Here we address the question whether IEC phenomena are possible in all-semiconductor superlattices, like EuS/PbS and GaMnAs/ GaAs, where the carrier concentrations are many orders of magnitude lower than in metals.

The nonmagnetic spacer in EuS/PbS SLs is a narrow gap semiconductor with electron concentration of the order of $10^{17} \mathrm{~cm}^{-3}$ to $10^{18} \mathrm{~cm}^{-3}$. For thin PbS layers $\left(d_{\mathrm{PbS}}<70 \AA\right)$ neutron reflectivity spectra, shown in Fig. 1, have revealed a pronounced maximum of magnetic origin at the position corresponding to the doubled structural SL periodicity, thus indicating the existence of antiferromagnetic (AFM) interlayer arrangements [3].

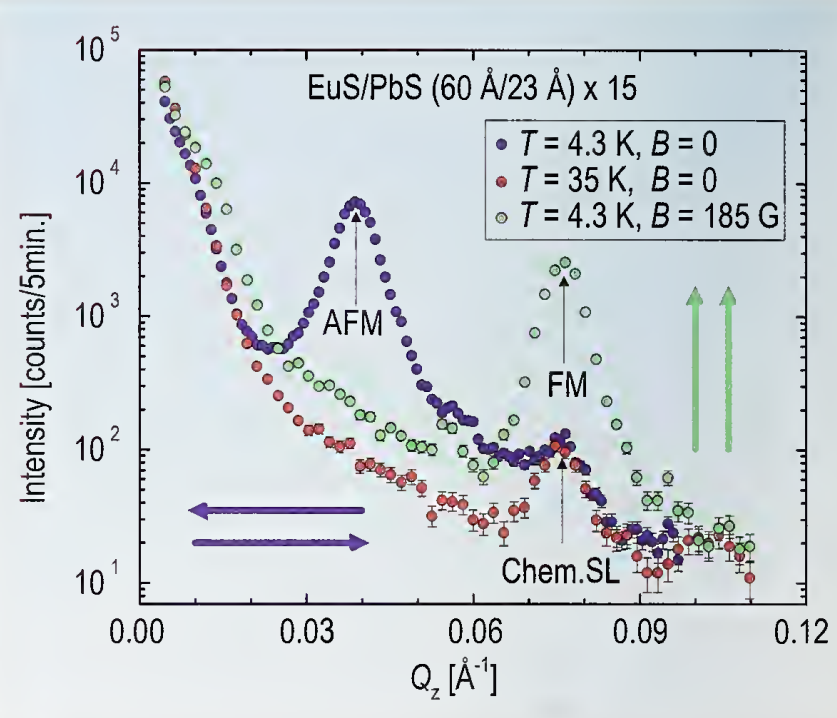

FIGURE 1. Unpolarized neutron reflectivity spectra for EuS/PbS SL with thin $(23 \AA)$ PbS spacer. Antiferromagnetic interlayer exchange coupling below $T_{\mathrm{c}}$ and at zero external field is clearly visible (blue curve). Applying a strong enough magnetic field ( $185 \mathrm{G}$ in this case) parallel to the SL surface forces all the EuS layer's magnetizations to ferromagnetic configuration (green curve). Above $T_{c}$ the system is nonmagnetic, the only Bragg peak comes from the chemical SL periodicity.

For much thicker PbS spacers $\left(d_{\mathrm{PbS}}>120 \AA\right)$ the only magnetic peaks visible in the reflectivity profiles, see e.g., Fig. 2, coincide with the chemical ones, thus leading to the conclusion that the magnetization vectors in adjacent EuS layers are parallel, which indicates FM IEC.

In the intermediate $\mathrm{PbS}$ thickness range (70 $\AA<d_{\mathrm{PbS}}<120 \AA$ ), both AFM and FM peaks are present. Polarized neutron analysis of these maxima gives evidence that the magnetization vectors of adjacent EuS layers are not colinear. Hence, the IEC found in EuS/PbS SLs has an oscillatory character similar to that occurring in metallic SLs, although the oscillation period is much longer than the one in metallic systems.

In order to confirm that the free carriers, present in the $\mathrm{PbS}$ layer in such a small amount, are the cause of the observed oscillatory IEC, a series of analogous measurements have been carried out on EuS/YbSe SLs. The structure and lattice constant of YbSe are the same as those of $\mathrm{PbS}$. In contrast to PbS, YbSe is a semi-insulator with a negligible carrier concentration. Neutron reflectivity profiles have shown no evidence of any interlayer coupling in the all 
A. Yu. Sipatov, V. Volobuev Kharkov State Polytechnical University Kharkov, Ukraine

H. Kepa, J. Kutner-Pielaszek, A. Twardowski Institute of Experimental Physics,

Warsaw University

Warsaw, Poland
T. Story, J. Sadowski Institute of Physics,

Polish Academy of Sciences

Warsaw, Poland

T. M. Giebultowicz

Physics Department

Oregon State University

Corvallis, OR 97331
C. F. Majkrzak

NIST Center for Neutron Research

National Institute of Standards and Technology

Gaithersburg, MD 20899-8562

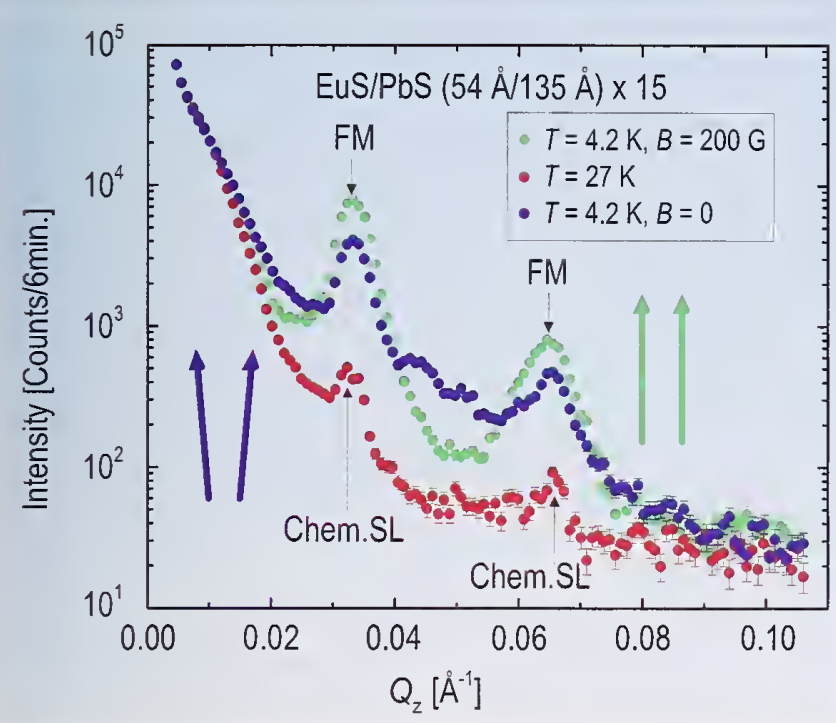

FIGURE 2. The sample with thick ( $135 \AA$ ) PbS layers is almost ferromagnetically coupled. Application of an external magnetic field enhances the FM Bragg peaks and lowers the intensity between them (at the AFM peak position).

investigated samples. That finding, together with the oscillatory character of coupling in SLs with PbS spacer, strongly points to the leading role of $\mathrm{PbS}$ free electrons in providing the necessary IEC mechanism, similar to that discovered in metallic multilayers.

Ferromagnetic ordering in GaMnAs is carrier (holes) induced; the Mn atoms, apart from being the magnetic element in the system, act also as acceptors providing the holes responsible for transferring exchange interactions between them. The details of the magnetic ordering, in particular its range, are still being disputed.

To address the latter issue, polarized neutron reflectometry has been performed on a number of GaMnAs/GaAs superlattices. Figure 3 shows an example of the obtained reflectivity profile in the vicinity of the first SL Bragg peak, for one of the samples. The very presence of the magnetic contribution to the structural SL Bragg peak is a strong confirmation of the FM IEC between consecutive GaMnAs layers. The absence of any spin-flip scattering shows that the sample is in a one-domain state, i.e., the FM ordering in $\mathrm{GaMnAs}$ is long range, and the sample is spontaneously saturated. The peak in (--) cross section, and its absence in

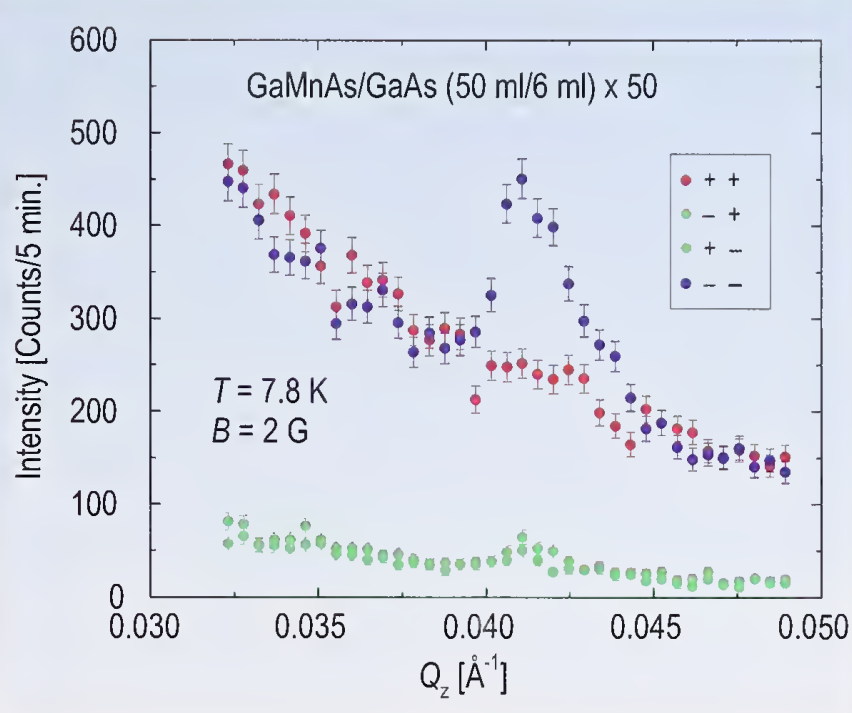

FIGURE 3. Polarized neutron reflectivity spectra for GaMnAs/GaAs superlattice.

the $(++)$, is proof that the magnetization is directed oppositely to the external magnetic guide field, hence the long range ordering has formed spontaneously, without the influence of the external field. More details can be found in Reference 4.

\section{References}

[1] T. Dietl, H. Ohno, F. Matsukura, J. Cibert, D. Ferrand, Science 287,1018 (2000),

[2] P. Bruno, Phys. Rev. B52, 411 (1995).

[3] H. Kepa, J. Kutner-Pielaszek, J. Blinowski, A. Twardowski, C. F. Majkrzak, T. Story, P. Kacman, R. R. Galazka, K. Ha, H. J. M. Swagten, W. J. M de Jonge, A. Yu. Sipatov, V. Volobuev, T. M. Giebultowicz, Europhys. Lett. 56, 54 (2001).

[4] H. Kepa, J. Kutner-Pielaszek, A. Twardowski, C. F. Majkrzak, J. Sadowski, T. Story, T. M. Giebultowicz, Phys. Rev. B64, 121302 (2001) 


\section{Pinpointing Chiral Structures with Front/Back Polarized Neutron Reflectometry}

$\mathbf{W}$ e have developed a new method of using polarized neutron reflectometry (PNR) to extract the structure of buried magnetic spirals in magnetic films. This technique improves upon earlier methods by being particularly sensitive to the presence of magnetic twists vis-à-vis structures in which the magnetization direction does not vary appreciably. Tracking the formation and growth of twists may solve a number of puzzles that hamper the development of magnetic thin film devices.

In collaboration with IBM scientists, we have applied the technique to a thin-film exchange-spring magnet and confirmed that the results may violate the current theory regarding the behavior of such magnets. It has been predicted that exchange-spring magnets, comprised of soft and hard ferromagnets in close proximity, are a composite that has a strong moment and does not readily demagnetize [1]. Therefore, exchange-spring magnets should give industry the ability to make much smaller permanent magnets for use in the magnetic recording devices, and elsewhere. As a side effect, when a small external magnetic field is opposed to that of the magnet, the portion of the soft ferromagnet farthest from the hard ferromagnet may twist into alignment with the field. When the field is removed, the soft ferromagnet untwists. The film provided by IBM consists of the hard ferromagnet $\mathrm{Fe}_{55} \mathrm{Pt}_{45}$ topped by the soft ferromagnet $\mathrm{Ni}_{80} \mathrm{Fe}_{20}[2]$.

Figure 1 shows a simplified diagram of the behavior predicted by current theories [1]. A magnetic field of $0.890 \mathrm{~T}$, provided by an electromagnet, is sufficient to align both the soft and the hard layers of our exchange-spring magnet, as shown on the left. When a modest reverse field (on the order of $0.025 \mathrm{~T}$ ) is applied to the exchange spring magnet, only the top of the soft layer will realign with the magnetic field. The hard layer remains pinned in the original direction, and a continuous twist is induced in the soft layer, as the direction of magnetization changes smoothly between the reverse field direction to the aligning field direction.

Although there are many alternatives to PNR to measure the magnetization, typically they measure only the average orientation of the magnetic spins, and cannot readily distinguish a spiral from a structure in which all the spins are canted with respect to an external field. PNR can extract the
Soft ferromagnet

Hard ferromagnet

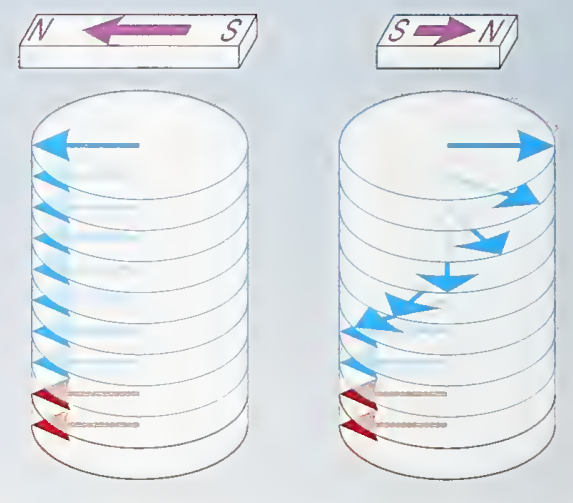

FIGURE 1. Model for field behavior of exchange-spring magnets. On the left the magnet has been aligned by a large external magnetic field. On the right a smaller field opposed to the first field causes a twist to form in the soft ferromagnet, while the hard ferromagnet remains aligned.

depth-dependence of the magnetic and chemical structure. We have studied the sample over a wide range of external magnetic fields, and can track the development of the spiral with field [3].

A PNR experiment begins with neutrons whose magnetic moments are aligned parallel $(+)$ or opposite $(-)$ to the external magnetic field. When the magnetization of the sample is perpendicular to this magnetic field, the neutron moment precesses as it interacts with the sample. When this happens the spin-flip (SF) reflectivities $R^{+-}$and $R^{-+}$are strong. If the magnetization of the sample is parallel to the external magnetic field, no precession occurs, but the nonspin-flip (NSF) reflectivities $R^{++}$and $R^{--}$will differ. The NSF reflectivities also provide information about the chemical structure of the film.

Our new modification of the PNR method greatly enhances the contrast between colinear and certain noncolinear magnetic structures [4]. We first measure the reflectivity with neutrons glancing off the front surface of the material, and then repeat with neutrons glancing off the back surface. The experiment is akin to holding the plane of the film up to a "magnetic mirror" to see whether the mirror image is the same as the original structure. In a colinear structure, all the spins are aligned along a common direction, and the mirror image is very much like the original structure. 


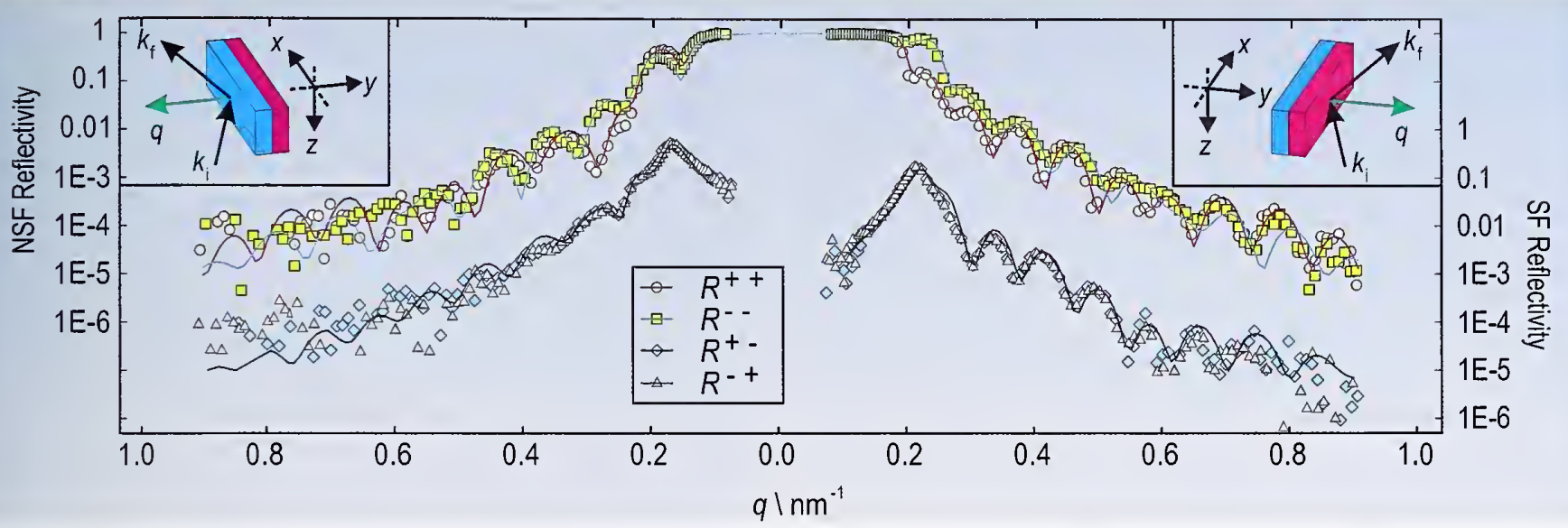

FIGURE 2. Reflectivity of a $\mathrm{Ni}_{80} \mathrm{Fe}_{20} \mathrm{IFe}_{55} \mathrm{Pt}_{45}$ bilayer. The front reflectivity is plotted on the right while the back reflectivity is plotted on the left. The SF reflectivities $R^{+*}$ and $R^{+^{+}}$are plotted against the right ordinate axis. The NSF reflectivities $R^{++}$and $R^{\cdots}$ are plotted against the left ordinate axis.

But the mirror image of a magnetic twist to the right is a magnetic twist to the left. Therefore, if the front and back reflectivities are significantly different, we can deduce the presence of a spiral. Fitting the data confirms the spiral's existence.

Figure 2 shows data collected at $0.026 \mathrm{~T}$ after aligning in $-0.89 \mathrm{~T}$. Fits to the data are shown as solid lines. The data from the front reflectivity are shown on the right, and the data from the back reflectivity are shown on the left. The spin-flip (SF) reflectivities $R^{+-}$and $R^{-+}$are plotted against the right-hand axis, which have been shifted relative to the NSF reflectivities $R^{++}$and $R^{--}$plotted against the left axis. At $q=0.2 \mathrm{~nm}^{-1}$, there is a splitting in the front NSF reflectivity that is much more pronounced than that of the back reflectivity at the same $q$. This is a hallmark of the spiral structure.

Figure 3 shows the magnetic structure that gives the excellent fit to the data plotted in Fig. 2. The location of the hard/soft interface is marked in Fig. 3. Surprisingly, we discover the spiral invades the hard ferromagnet even at extremely low fields. Current theory predicts that when this occurs, the soft ferromagnet will not be able to untwist fully. Yet, other magnetic studies show that our exchange-spring magnet does untwist when this field is removed. Thus, our PNR measurements have identified a shortcoming of current theory.
With this new technique, NIST is now able to better characterize the magnetic properties of thin films, which can improve the capability and reliability of industrial devices for magnetic recording and sensing.

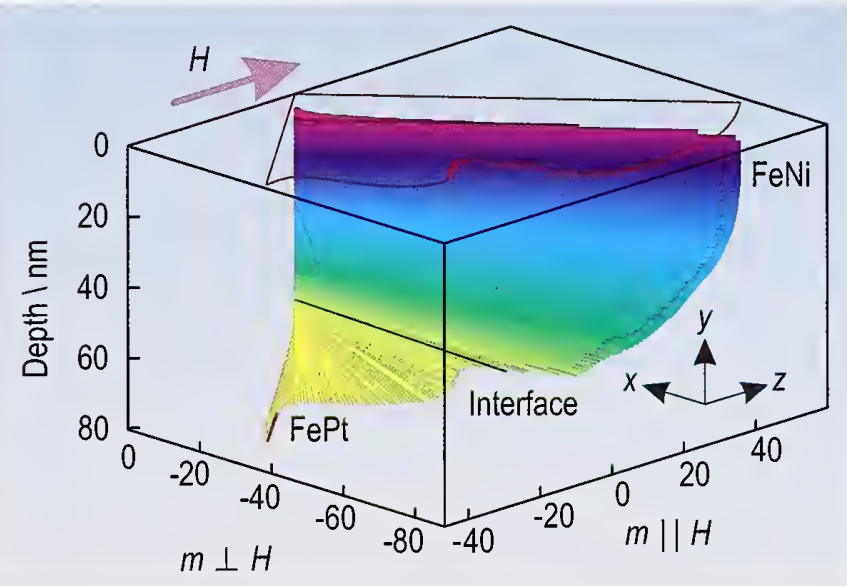

FIGURE 3. Fitted magnetization of the data presented in Fig. 2. The front of the sample is at a depth of $0 \mathrm{~nm}$ and the back is at a depth of $70 \mathrm{~nm}$. The red curve is a projection of the magnetic structure into the plane of the front surface.

\section{References}

[1] E. F. Kneller and R. Hawig, IEEE Trans. Magn. 27, 3588 (1991).

[2] O. Hellwig, J. B. Kortright, K. Takano, and E. E. Fullerton, Phys. Rev. B62, 11694 (2000).

[3] K. V. O'Donovan, et al., in preparation.

[4] K. V. O'Donovan, et al., submitted to Applied Physics A. 


\section{The Nature of Vibrational Softening in $\alpha=$ Uranium}

$\mathrm{T}$ he standard textbook explanation for phonon softening with increasing temperature in a single phase is that the interatomic potentials are not perfectly harmonic, but it has been suggested that phonon softening can also occur if the potential itself can change with temperature, while remaining harmonic. For example, a large softening of the vibrational properties of $\alpha$-uranium has been observed [1] that cannot be explained in terms of the anharmonic lattice contribution alone. In the present work a harmonic contribution to the phonon softening is made evident by treating inelastic neutron scattering spectra as an expansion of the vibrational power spectrum of the atomic motion.

Earlier diffractometry measurements by Lawson $\mathrm{et} \mathrm{al}$, [2] suggested that the Debye temperature decreased by $40 \%$ in going from $300 \mathrm{~K}$ to the $\beta$-phase transition temperature at $940 \mathrm{~K}$. In terms of entropy this corresponds to an additional $\Delta S=-3 k_{\mathrm{B}} \ln (0.6)=1.5 k_{\mathrm{B}}$ /atom. The usual thermodynamic argument is that this increase in vibrational entropy compensates for the elastic energy generated by thermal expansion. However, from heat capacity data, the entropy needed to compensate for the elastic energy is nearly an order of magnitude too small at $0.16 k_{\mathrm{B}}$ /atom.

All experiments used uranium powder. High temperature measurements were made using the Fermi-Chopper Spectrometer (FCS) at the NCNR. Low temperature measurements were performed with the Low Resolution Medium Energy Chopper Spectrometer (LRMECS) at Argonne National Laboratory. Figure 1 shows the phonon density of states obtained from the measured spectra, corrected for the incoherent multiphonon scattering using a procedure described elsewhere [3]. There is a redistribution of intensity in the main features at $\approx 8 \mathrm{meV}$ and $\approx 12 \mathrm{meV}$, with the higher energy peak gaining extra weight with increasing temperature. These features also show an overall softening of around one meV for every $200 \mathrm{~K}$ increase in temperature.

The Q-summed one phonon scattering function was used to calculate a quantity proportional to the square of the power spectrum and hence to the average potential energy per oscillator, $\langle U\rangle$. In Fig. 2 we show $\langle U\rangle$ for $\alpha$-uranium at the four highest temperatures scaled so that the lowest point is at the harmonic energy $k_{\mathrm{B}} T / 2$. The effects of anharmonicity on $\langle U>$ would be evident as a nonlinearity in

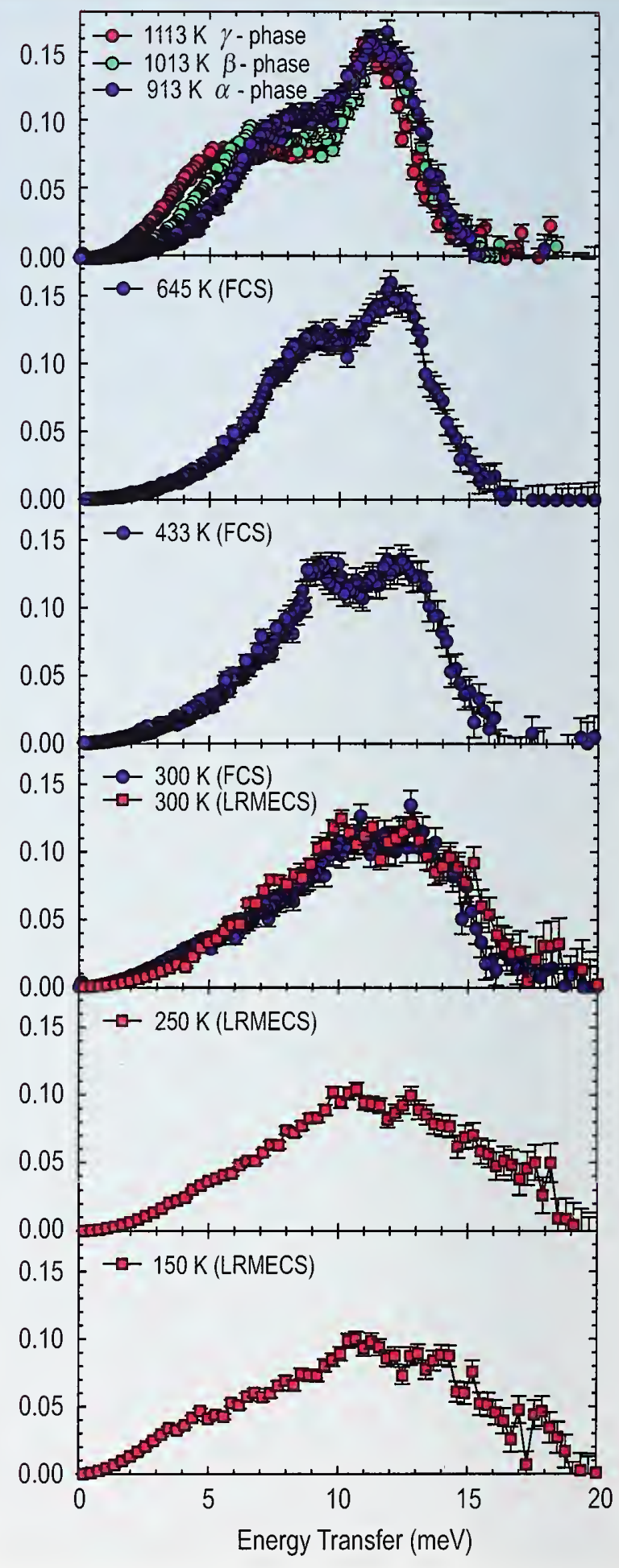

FIGURE 1. The phonon density of states of uranium. Data from $300 \mathrm{~K}$ and above were obtained from spectra acquired with FCS. Data from $300 \mathrm{~K}$ and below were measured on LRMECS. 
M. E. Manley and B. Fultz

California Institute of Technology

Pasadena, CA 91125

M. E. Manley, R. J. McQueeney,

W. L. Hults, J. L. Smith, and D. J. Thoma

Los Alamos National Laboratory

Los Alamos, NM 87545
C. M. Brown

NIST Center for Neutron Research

National Institute of Standards and Technology

Gaithersburg, MD 20899-8562

and

University of Maryland

College Park, MD 20742
R. Osborn

Argonne National Laboratory

Argonne, IL 60439

J. L. Robertson

Oak Ridge National Laboratory

Oak Ridge, TN 37831 the plot of $\langle U\rangle$ vs. temperature. For comparison, attempts were made to calculate the temperature dependence of the potential energy of Morse and Lennard-Jones potentials with the appropriate vibrational softening for uranium [1]. The result shown in Fig. 2 indicates that, in the high temperature limit, the potential energy has nearly a linear dependence on temperature, i.e., the phonon softening in $\alpha$-uranium occurs while the potential remains primarily harmonic. Evidently the interatomic force constants are decreasing with increasing temperature. Since the force constants originate from the change of the electronic energy with atom displacements, it must be that thermal excitations of the electronic states are altering the force constants.

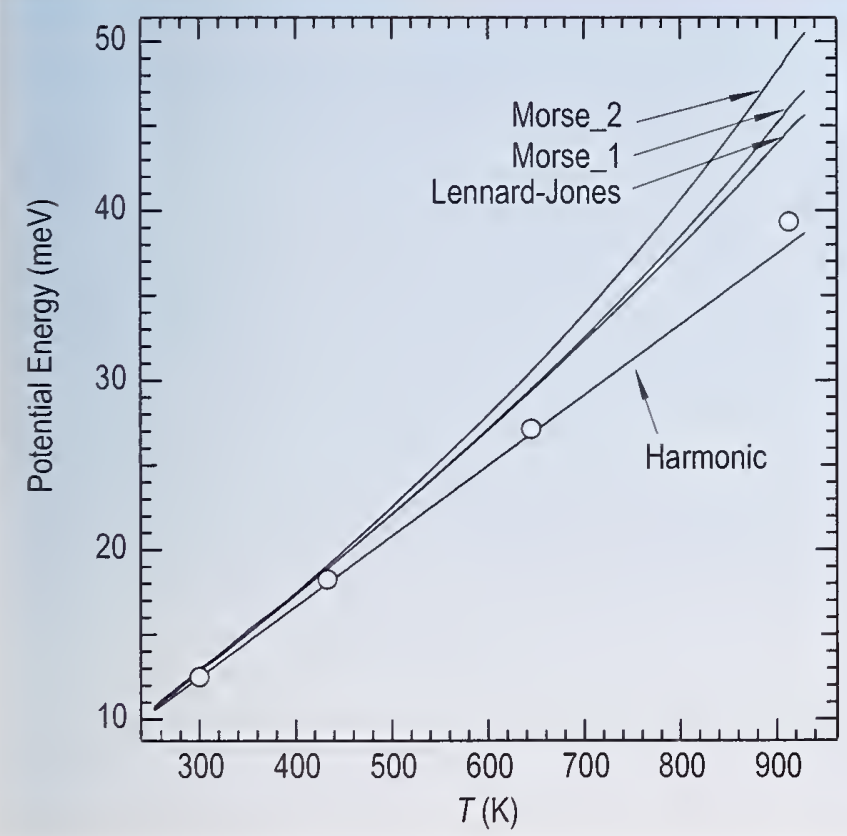

FIGURE 2. Vibrational potential energy of $\alpha$-uranium (open circles). The Lennard-Jones, Morse_ 1 and Morse_2 curves were calculated from potentials described in the text. The Harmonic curve is the result for a harmonic potential in the classical limit.

The phonon density of states of the three solid state phases of uranium, orthorhombic $(\alpha)$, tetragonal $(\beta)$ and body centered cubic $(\gamma)$ are compared in the top panel of Fig. 1. The $\gamma$-uranium phonon density of states was statistically identical at $1113 \mathrm{~K}$ and $1213 \mathrm{~K}$. Evidently, the thermal softening mechanism seen in $\alpha$-phase does not operate in the $\gamma$-phase. The $\beta$-phase was not stable over a wide enough temperature range to obtain a reliable temperature dependence. The phonon softening between each phase accounted for vibrational entropy changes of $0.15 \pm 0.01 k_{\mathrm{B}} /$ atom and $0.20 \pm 0.01 k_{\mathrm{B}}$ /atom for the $\alpha$ to $\beta$ and $\beta$ to $\gamma$ transitions, respectively. Both of these values make up only about $35 \%$ to $40 \%$ of the total entropy changes predicted from latent heat measurements: $\left(S^{\beta}-S^{\alpha}\right)_{\text {tot }}=0.37 k_{\mathrm{B}} /$ atom and $\left(S^{\gamma}-S^{\beta}\right)_{\text {tot }}=0.55 k_{\mathrm{B}} /$ atom. The remaining $60 \%$ of the entropy increases must be electronic in origin. So not only does the phonon softening disappear in the high temperature $\gamma$ phase, but also it does so with a large increase in electronic entropy.

Electronic band structure calculations used to predict phonon frequencies are based on the assumption that thermal effects can be neglected when compared to volume effects. The actinides, however, show the need for more sophisticated treatments of the role of temperature on interatomic interactions.

\section{References}

[1] M. E Manley, B. Fultz, R. J. McQueeney, C. M. Brown, W. L. Hults, J. L. Smith, D. J. Thoma, R. Osborn, J. L. Robertson, Phys. Rev. Lett. 86, 3076 (2001).

[2] A. C. Lawson, B. Martinez, J. A. Roberts, B. I. Bennett and J. W. Richardson, Jr., Phil. Mag. B 80, 53 (2000).

[3] M. E. Manley, R. J. McQueeney, J. L. Robertson, B. Fultz and D. A. Neumann, Phil. Mag. Lett. 80, 591 (2000). 


\section{Native and Partially Unfolded Proteins: Neutron Inelastic Scattering and Simulations}

D roteins are long chain-like molecules that are intricately folded. As they function they unfold in specific ways. How proteins can quickly refold without tangling remains a central mystery in molecular biology. Partially folded proteins provide an approach to this problem, and are also important in cell functioning. The approach is that stable partially folded states have been shown to resemble the kinetic folding intermediate states along the proteinfolding pathway. A molten globule (MG) is a compact partially folded protein, with a backbone resembling the completely folded protein, but lacking the extensive specific side-chain packing interactions of the native-like (i.e., properly folded) structure. A full understanding of the mechanism of protein folding requires the knowledge of the structures, relative energetics, and dynamics of the species populating the folding pathway. Incoherent inelastic neutron scattering (INS), which makes use of the large incoherent cross section of hydrogen nuclei, is a technique well suited to the study of protein internal molecular motion on the picosecond time scale. Molecular dynamics (MD) simulations, on the other hand, are a potentially valuable tool for interpreting neutron data on proteins [1,2], and may provide atomic level description of the motions taking place.

In this study, we have explored the dynamics of bovine $\alpha$-lactalbumin, a calcium-binding protein, in the native and molten globule states, using both techniques. INS data were collected on the Disk-Chopper Spectrometer at $\approx 32 \mu \mathrm{eV}$ resolution for native and molten globular bovine $\alpha$-lactalbumin. The simulations results were generated from $\approx 1$ ns constant temperature and pressure MD trajectories. The model systems consisted each of a single protein monomer, immersed in a large water box

$(60 \AA \times 50 \AA \times 80 \AA)$. For the native state, the $\alpha$-lactalbumin configuration was initiated from the crystal structure. The model molten globule state was generated by partially unfolding the protein at $500 \mathrm{~K}$ at atmospheric pressure until the radius of gyration reached the experimental value determined for the molten globule state (10\% expansion). A $1 \mathrm{~ns}$ trajectory was then generated at $300 \mathrm{~K}$ for data analysis and comparison with experiment.

Figure 1 shows the structure of the $\alpha$-lactalbumin in the native and molten globule states obtained from the MD

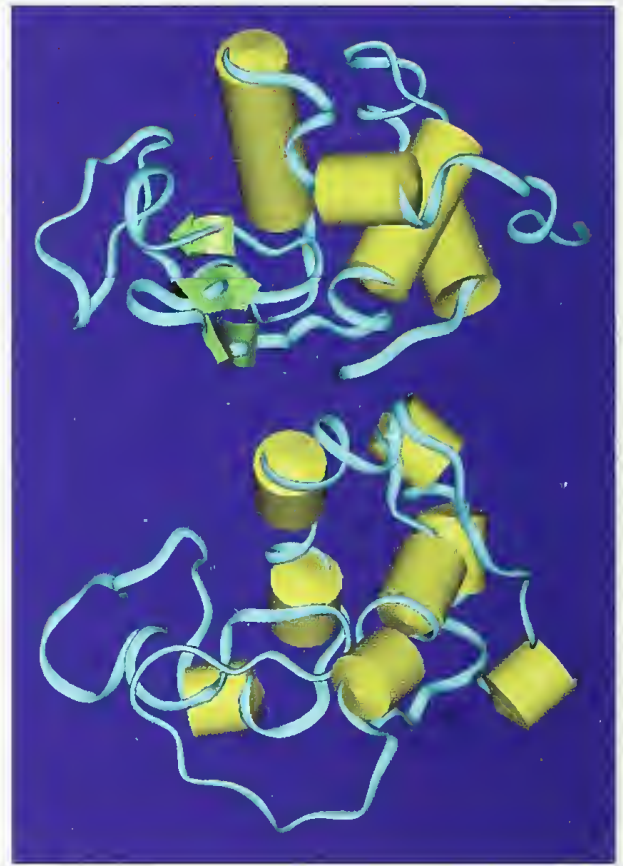

FIGURE 1. $\alpha$-lactalbumin structure in the native (top) and molten globule (bottom) state from MD simulations. Note the loss of the $\beta$-domain structure, with most the $\alpha$ domain (helix) being conserved.

simulations in solution at $300 \mathrm{~K}$. The overall structural change, manifested by the conservation of the helical $\alpha$-domain and the loss of the $\beta$ sheets in the $\beta$-domain, is consistent with NMR data [3]. We therefore regard this structure as a reasonable model of a member of the MG ensemble for comparison with the INS data.

In Fig. 2 we report the incoherent structure factors $S(Q, \omega)$ as a functions of energy transfer $\hbar \omega$ for selected momentum transfer $Q$. measured and calculated from the MD simulations trajectories. In both systems the agreement with experiment is remarkable, assessing the ability of the simulations to reproduce the picosecond dynamics of the protein. In agreement with previous INS data collected at lower resolution [4], the molten globule has a broader quasielastic spectra compared to the native state, which indicates that the protein atoms are more mobile in the MG state. The additional motion is large enough to be detected at the length scale probed by the DCS spectrometer, i.e., in the range $3 \AA$ to $60 \AA$.

To investigate the relationship between the structure of the protein and the motion of its side chains, we have used the MD trajectories to follow the motion of these side chains 
M. Tarek

NIST Center for Neutron Research

National Institute of Standards and Technology

Gaithersburg, MD 20899-8562 and

Center for Molecular Modeling and

Department of Chemistry, University of Pennsylvania

Philadelphia, PA 19103
D. J. Tobias

Department of Chemistry

University of California

Irvine, CA 92697-632
Z. Bu and D. A. Neumann

NIST Center for Neutron Research

National Institute of Standards and Technology Gaithersburg, MD 20899-8562

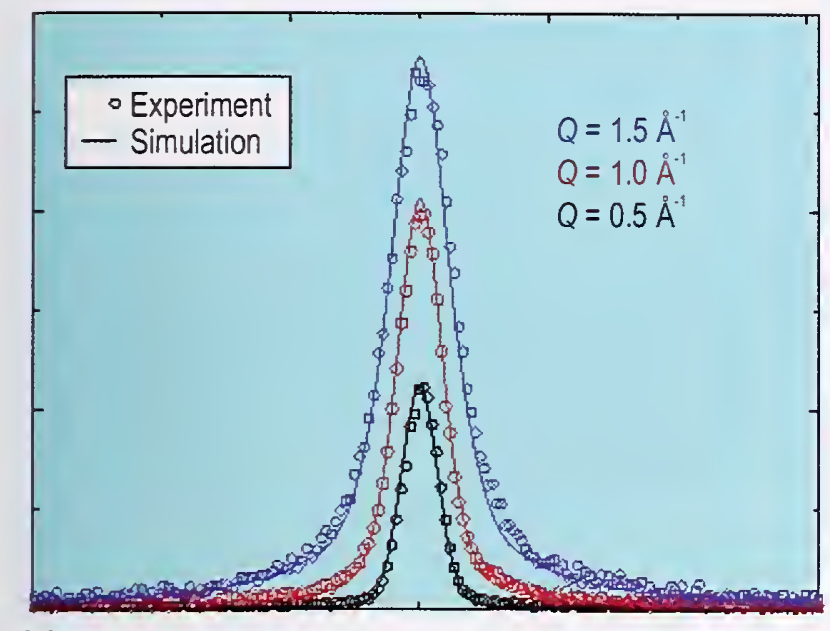

.0 .3

$-0.1$

0.1

0.3

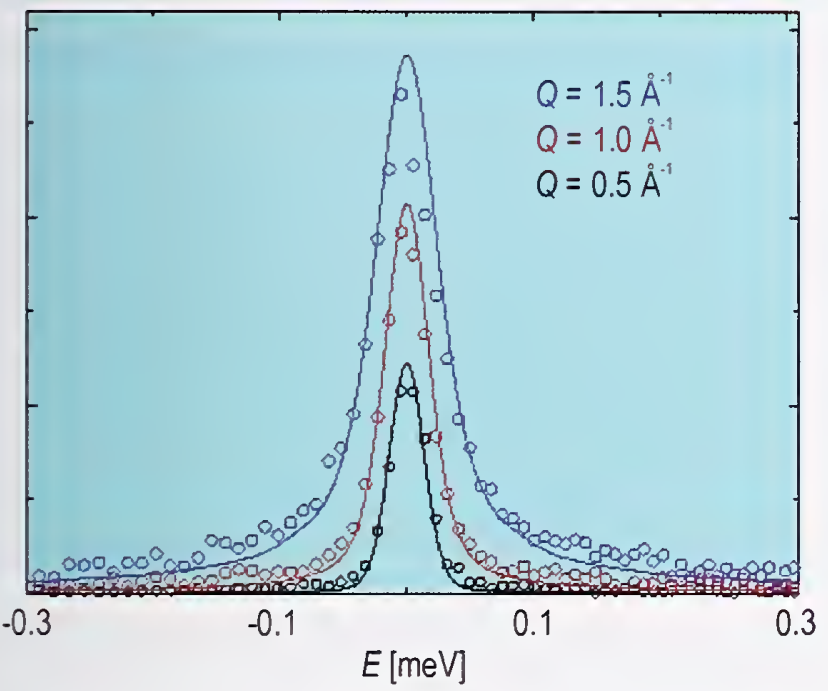

FIGURE 2. Incoherent structure factor $S(Q, \omega)$ at selected wavevectors $Q$, measured and calculated from the simulation for the native (top) and molten globule (bottom) states.

quantitatively. Figure 3 reports the fluctuations per residue along the backbone for the native and the molten globule states, as a function of residue. These correspond to the $100 \mathrm{ps}$ root-mean-square amplitudes of motion of the backbone atoms, averaged over the simulation trajectories.

First for the native state, the backbone fluctuations appear to correlate with the secondary structure of the protein, showing much smaller amplitudes in the $\alpha$-helix and $\beta$-sheet regions. For the molten globule state, a significant increase in the protein fluctuation amplitudes is observed, in agreement with the broadening of the INS spectra.

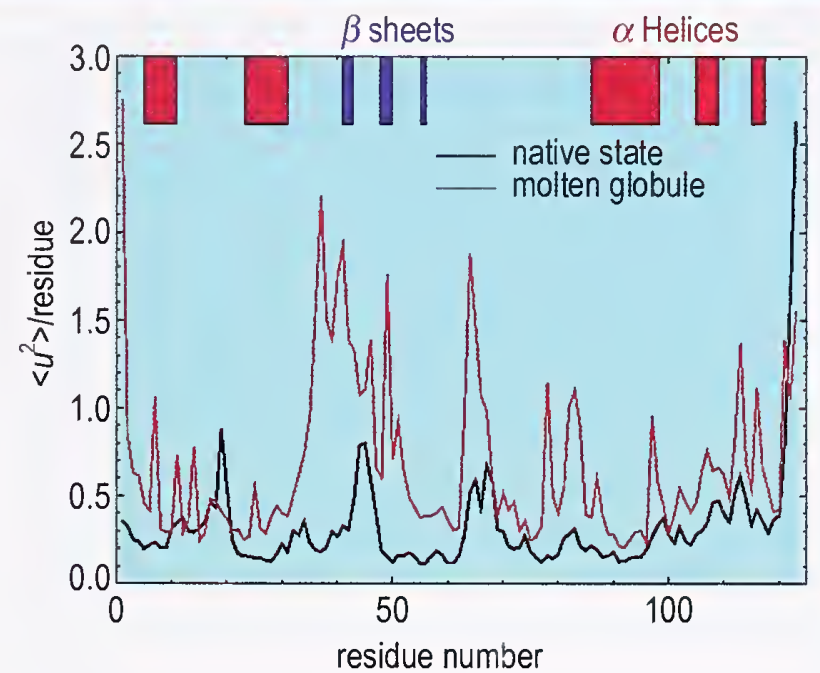

FIGURE 3. 100 ps mean squared fluctuations of the backbone atoms computed from the MD simulations of the native state (black) and the molten globule state (red).

Figure 3 shows clearly that most of the additional motion occurs in the region of the protein that "unfolds," i.e., the $\beta$-domain. The results indicate also that the motion of the side chains is by no means homogeneous. Differences in amplitudes up to 10 folds are observed between parts of the proteins, regardless of exposure to the solvent.

The MD study of the dynamics of partially folded states of the protein should in principle be extended to explore a large portion of the conformational space, i.e., be extended to a large ensemble of non-native conformers. At any rate, the present results, though limited, combined with neutron experiments are believed to capture the essence of the structural and dynamical changes taking place as the protein partially unfolds. They demonstrate the utility of the MD simulations for qualitatively elucidating the dynamical behavior of native and non-native proteins at the atomic level.

\section{References}

[1] M. Tarek, G. J. Matryna, and D. J. Tobias, J. Amer. Chem. Soc. 122, $10450(2000)$

[2] M. Tarek and D. J. Tobias. Biophys. J. 79, 3244 (2000).

[3] J. Baum et al., Biochemistry, 28, 7 (1989).

[4] Z. Bu et al., J. Mol. Biol. 301, 525 (2000). 


\section{Atomic Motions in Confined Polymer Films}

aterial properties at surfaces and interfaces often deviate from their bulk values. An example is viscoelastic properties that are important in many applications such as chemically amplified photoresists, lubricants. adhesives, etc. A viscosity increase of a liquid polymeric film, like those used for hard disc lubricants, could have deleterious consequences. Likewise. a viscosity decrease in a thin photoresist film could enhance photoacid diffusion and create image blur. It is clearly important to characterize the molecular level mobility in thin polymer films. Of particular interest are changes in the glass transition temperature $T_{\mathrm{g}}$, since the glass transition corresponds to a sharp change in viscosity.

It is well known that atomic thermal motions lead to a decrease in the intensity of the elastically scattered neutrons. In a harmonic solid the intensity is related to the amplitude of the motion through the Debye-Waller factor, $I_{\text {inc }}(Q) \sim \exp \left(-1 / 3 Q^{2}<u^{2}>\right)$, where $Q$ is the scattering vector and $\left\langle u^{2}\right\rangle$ is the mean-square atomic displacement. In a logarithmic plot of $I_{\text {inc }}$ as a function of $Q^{2},<t^{2}>$ is proportional to the slope of a linear fit to the data. This approximation has proven very useful in studying the dynamics of polymers, both synthetic [1] and biological [2], despite the fact that their atomic motions are typically anharmonic.

The elastic incoherent neutron scattering intensities can be measured as a function of temperature on the NCNR's High Flux Backscattering Spectrometer (HFBS), using the "Fixed Window" mode. Polycarbonate (PC) films, seen in Fig. 1, are spin cast onto Si wafers, sectioned into small rectangles, and stacked into an Al sample cell. The scattering from the Si substrates and Al cell are negligible because their Bragg peaks are beyond the $Q$ range of the instrument $\left(0.25 \AA^{-1}<Q<1.75 \AA^{-1}\right)$; the scattering is dominated by the large incoherent cross-section of the amorphous PC.

Ideally there should be $200 \mathrm{mg}$ of PC per cell to maximize the signal and minimize multiple scattering effects ( $90 \%$ transmission). For the Si-supported films we are limited by $0.5 \mathrm{mg}$ to $10 \mathrm{mg}$ of PC per cell and a thus obtain a very weak signal ( $99 \%$ transmission). However, the HFBS is sufficiently sensitive to observe this signal, as seen in Fig. 2 where the temperature dependence of the elastic scattering is shown. The relative magnitude of the intensity

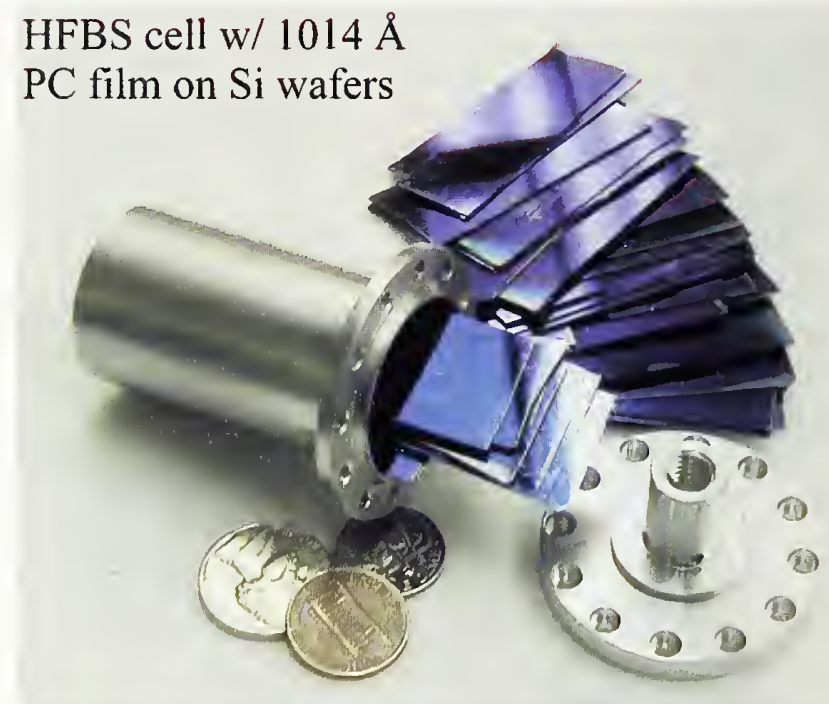

FIGURE 1. A stack of $1014 \AA$ PC films (notice the blue tint) on thin slabs of Si ready to go into the HFBS spectrometer.

change diminishes with decreasing film thickness, a clear indication of reduced atomic mobility in the thin PC films. Given the HFBS energy resolution of $0.85 \mu \mathrm{eV}$, these intensity changes reflect changes in atomic motions at frequencies of $200 \mathrm{MHz}$ or faster.

This reduced mobility is qualitatively reflected in Fig. 3 as strong suppression of $\left\langle u^{2}\right\rangle$. We emphasize qualitatively because $\left\langle u^{2}\right\rangle$ arises from a harmonic approximation. Nevertheless, there is a clear reduction in the amplitudes of the thermal motions, both above and below the calorimetric $T_{\mathrm{g}}$, as the film thickness approaches the root-mean-square (rms) end-to-end length of PC $\left(R_{\mathrm{ee}}=160 \AA\right)$.

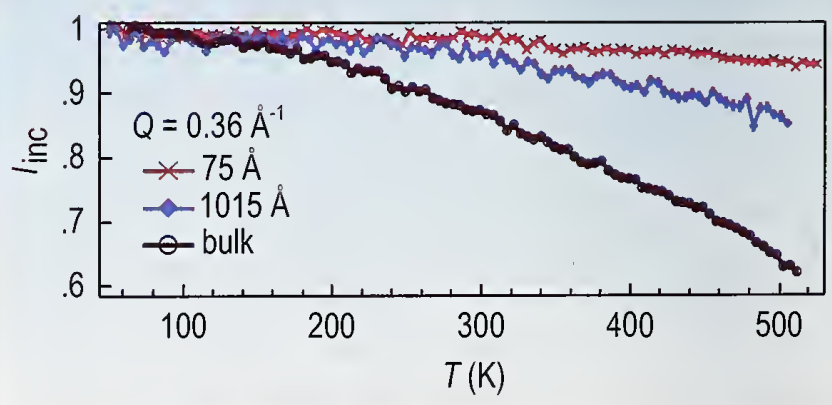

FIGURE 2. The normalized elastic intensities in the HFBS detector at $Q=0.36 \AA^{-1}$. The decrease in intensity at high temperatures reflects the presence of atomic motion in the thin polymer films. 
C. L. Soles, J. F. Douglas, and Wen-li Wu

Polymers Division

National Institute of Standards and Technology

Gaithersburg, MD 20899-8541

\section{R. M. Dimeo}

NIST Center for Neutron Research

National Institute of Standards and Technology

Gaithersburg, MD 20899-8562

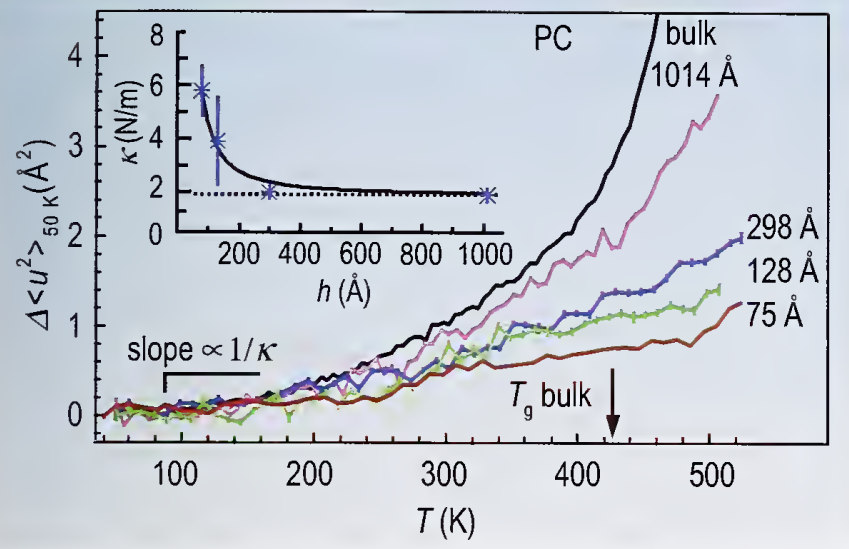

FIGURE 3. $\left\langle u^{2}\right\rangle$ as a function of temperature for the PC thin films. The inset reveals a stiffening of the harmonic (low temperature) force constant $\kappa$ with decreasing film thickness $h$.

At temperatures below $200 \mathrm{~K},\left\langle u^{2}\right\rangle$ is small and the harmonic approximation is reasonable. In this regime the inverse of the slope of $\left\langle u^{2}\right\rangle$ vs. $T$ is proportional to an elastic force constant $\kappa$. The inset of Fig. 3 displays a dramatic stiffening of $\kappa$ with confinement. This implies that the atoms or molecules in PC become strongly caged by their environment in the thinner films.

Similar $\left\langle u^{2}>\right.$ measurements on thin films of polymethyl methacrylate (PMMA; $R_{\mathrm{ee}}=915 \AA$ ) and polyvinyl chloride (PVC; $R_{\mathrm{ee}}=437 \AA$ ) are shown in Figs. 4(a) and 4(b). There is a suppression of $\left\langle u^{2}\right\rangle$ with decreasing film thickness above the bulk $T_{\mathrm{g}}$ that is qualitatively consistent with the PC films. However, below the $T_{\mathrm{g}}$ for bulk PMMA the suppression is less pronounced, and not observed in the PVC films. Careful inspection of Figs. 3 and 4 suggests a relation between the magnitude of $\left\langle u^{2}\right\rangle$ at $T_{\mathrm{g}}$ in the bulk and the degree of $\left\langle u^{2}\right\rangle$ suppression in the thin glassy films.

Bulk PC has the largest $\left\langle u^{2}\right\rangle$ at $T_{\mathrm{g}}$, indicating extensive mobility. In fact, PC is well known for its segmental mobility in the glassy state, which is in part related to its superior impact resistance. Molecular mobility helps dissipate strain energy. (PC is used in bullet-proof glass.) This extensive mobility is also susceptible to thin film confinement, as also seen through the stiffening of the elastic constants from $1.9 \mathrm{~N} / \mathrm{m}$ in bulk PC to $5.8 \mathrm{~N} / \mathrm{m}$ in the $75 \AA$ film. At the other extreme, bulk PVC has the smallest $\left\langle u^{2}\right\rangle$ at $T_{\mathrm{g}}$, indicating a lack of motions to be affected by confinement. This is also consistent with the strong intermolecular
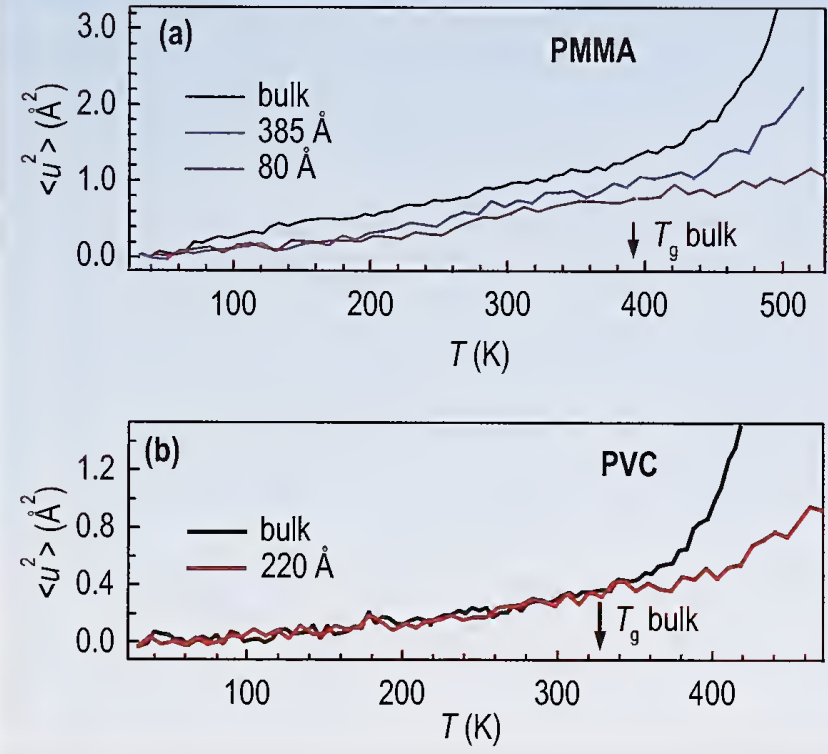

FIGURE 4. $\left\langle u^{2}\right\rangle$ as a function of thickness and temperature for similar PMMA (a) and PVC (b) films.

caging at low temperatures; both bulk PVC and the $220 \AA$ film have large elastic force constants of $4.6 \mathrm{~N} / \mathrm{m}$. This lack of mobility reflects the observation that PVC is a brittle polymer that needs high levels of plasticization for practical use. Only when PVC is taken above the bulk $T_{\mathrm{g}}$, where long-range motions dominate, do the effects of confinement become evident.

In short, we have seen two different responses to thin film confinement. While there is a general suppression of $\left\langle u^{2}\right\rangle$ and the fast dynamics, polymers with greater mobility are more susceptible to confinement, especially below the calorimetric $T_{\mathrm{g}}$. In contrast, polymers with strongly localized motions appear bulk-like, even in highly confined glassy films. More details of these studies can be found in our upcoming publications [3-5].

\section{References}

[1] B. Frick, D. Richter, Science 267, 1939 (1995).

[2] G. Zaccai, Science 288, 1604 (2000).

[3] C. Soles, J. Douglas, W. Wu, R. Dimeo, submitted to Phys Rev E.

[4] C. Soles, J. Douglas, W. Wu, H. Peng, D. Gidley, in preparation.

[5] C. Soles, E. Lin, J. Lenhart, R. Jones, W. Wu, D. Goldfarb, M. Angelopoulos, J. Vac. Sci. Tech. B, in press. 


\section{Tuning the Properties of Carbon Nanotubes by Deformation}

arbon nanotubes continue to surprise scientists with their novel properties. Recently we have discovered many intimate relationships between structural deformation and the properties of single-walled nanotubes (SWNT) that could be important in technological applications $[1,3]$. These observations were made using state-of-the-art firstprinciples total energy calculations within the pseudopotential and the generalized gradient approximations (GGA). Here we present a brief review of our findings.

SWNTs are basically rolled graphite sheets which are characterized by two integers $(n, m)$ defining the rolling vector of graphite. Therefore, the electronic properties of SWNTs, to first order, can be deduced from that of graphite by mapping the band structure from a $2 \mathrm{D}$ hexagonal lattice onto a cylinder. Such an analysis indicates that $(n, n)$ "armchair" nanotubes are always metallic and $(n, 0)$ zigzag nanotubes are metallic only if $n$ is an integer multiple of three. From our first-principles calculations we find that these electronic properties can be significantly modified by radial strain, which distorts the circular cross section to an elliptical one.

Figure 1 shows that the energy gap of an insulating SWNT decreases and eventually vanishes at an insulatormetal transition with increasing applied elliptical strain. The density of states at the Fermi level $N\left(E_{\mathrm{F}}\right)$, of a metallized SWNT increases with increasing deformation. More interestingly, the elliptical deformation necessary to induce metallicity is found to be in the elastic range. Therefore, all strain induced changes in electronic and also in mechanical properties are reversible.

Another significant effect of the elliptical distortion of SWNTs is the change in the uniformity of their charge distribution. This, in turn, imposes changes in the chemical reactivity and hence on the interaction of the tube surface with foreign atoms and molecules. To demonstrate this, we calculate the binding energies of $\mathrm{H}$ and a simple metal atom, $\mathrm{Al}$, on SWNTs as functions of nanotube radius $(R)$, type, and elliptical deformation. We find that the binding energy, $E_{\mathrm{B}}$, is positive (i.e., stable) and follows a remarkably simple $1 / R$ scaling for $(n, 0)$ SWNTs for both $\mathrm{H}$ and $\mathrm{Al}$, even though $\mathrm{H}$ prefers to sit on top of $\mathrm{C}$ atoms and $\mathrm{Al}$ favors the hollow sites (i.e., top of hexagons). Increasing binding energy with
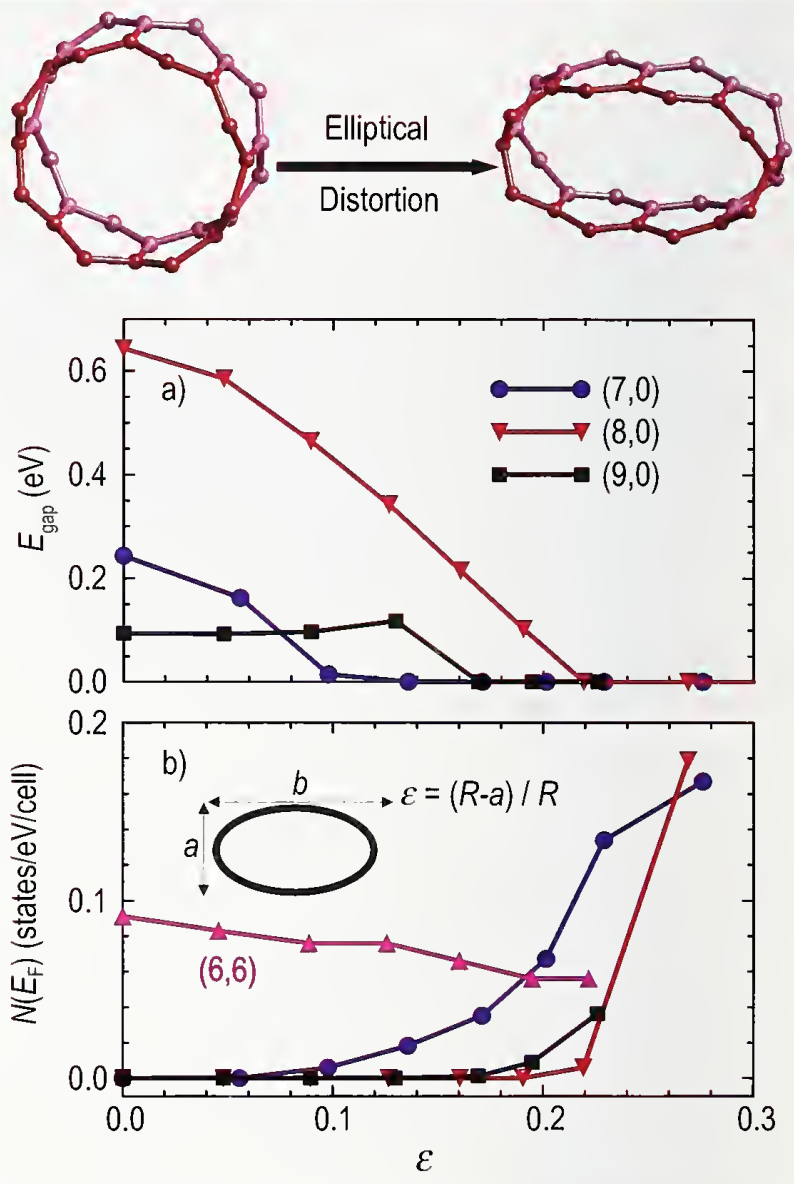

FIGURE 1. Variation of the energy band gap $E_{\text {gap }}$ (a) and density of the states at the Fermi energy (b) as a function of elliptical deformation.

increasing curvature (i.e., decreasing $R$ ) suggests that $E_{\mathrm{B}}$ can be modified by elliptical deformation.

Figure 2 shows the variation of the binding energy $E_{\mathrm{B}}$ of a single hydrogen atom adsorbed on the sharp and flat edges of the $(8,0)$ surface with elliptical distortion. With increasing distortion, the binding energy of the sharp site increases, while it decreases for the flat site, creating an energy difference of $\approx 1.1 \mathrm{eV}$ at the distortion value $\varepsilon=0.3$. This value is $44 \%$ of the binding energy of $\mathrm{H}$ on the undeformed SWNT.

The binding energy of $\mathrm{Al}$ exhibits a behavior similar to that of $\mathrm{H}$, despite $\mathrm{H}$ and $\mathrm{Al}$ favoring different sites on the $(8,0)$ tube. Elliptical deformation increases the binding energy of both the flat and sharp sites for $\mathrm{Al}$, given an energy difference of $0.5 \mathrm{eV}$ at the deformation $\varepsilon=0.3$. It is 
T. Yildirim

NIST Center for Neutron Research

National Institute of Standards and Technology

Gaithersburg, MD 20899-8562

\section{O. Gulseren}

NIST Center for Neutron Research

National Institute of Standards and Technology

Gaithersburg, MD 20899-8562 and

Department of Materials Science and Engineering

University of Pennsylvania

Philadelphia, PA 19104-6202

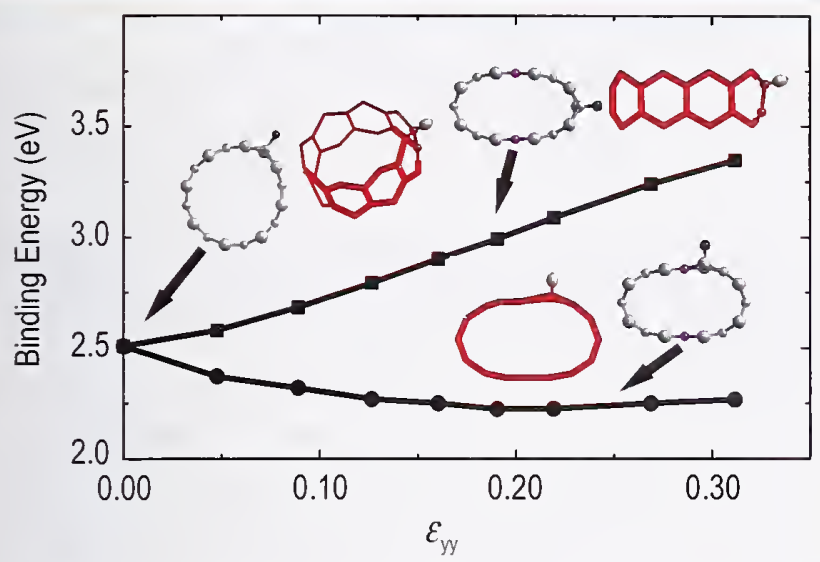

FIGURE 2. Binding energies of the sharp (top) and the flat sites (bottom) versus elliptical deformation for a hydrogen adsorbed on a $(8,0)$ SWNT.

remarkable that $\mathrm{Al}$, which is not bound to the graphite, can be adsorbed at the sharp site of the distorted SWNT with a binding energy of $1.8 \mathrm{eV}$. Hence, we conclude that not only band gap engineering but also chemical reactions taking place on the surface of a SWNT can be engineered through radial deformation.

Explanation of this remarkable change of the binding energy with elliptical deformation is sought in the electronic structure of SWNTs. Figure 3 shows the response of the first conduction band to elliptical distortion. It is clear that the distortion disturbs the uniformity of the charge distribution of the SWNT and pushes the chemically most active electrons from the flat to the sharp site of the nanotube, increasing the adsorption energy of the sharp site significantly. Surprisingly the effect of distortion on metallic armchair $(n, n)$ nanotubes is found to be very small due to the metallic bands of the tubes [2]. This could be important in selective functionalization of nanotubes. See Ref. 1 for animation of this effect.

We believe that the tunable electronic and chemical properties reported here can have important implications for metal coverage and for selective adsorption and desorption of atoms and molecules on carbon nanotubes. This could allow the fine tuning of the properties of SWNTs via reversible deformation and can ultimately lead to a wide variety of technological applications such as variable metalinsulator junctions, quantum wells, catalysts, hydrogen storage devices, magnetic tubes (by absorbing magnetic ions), etc.
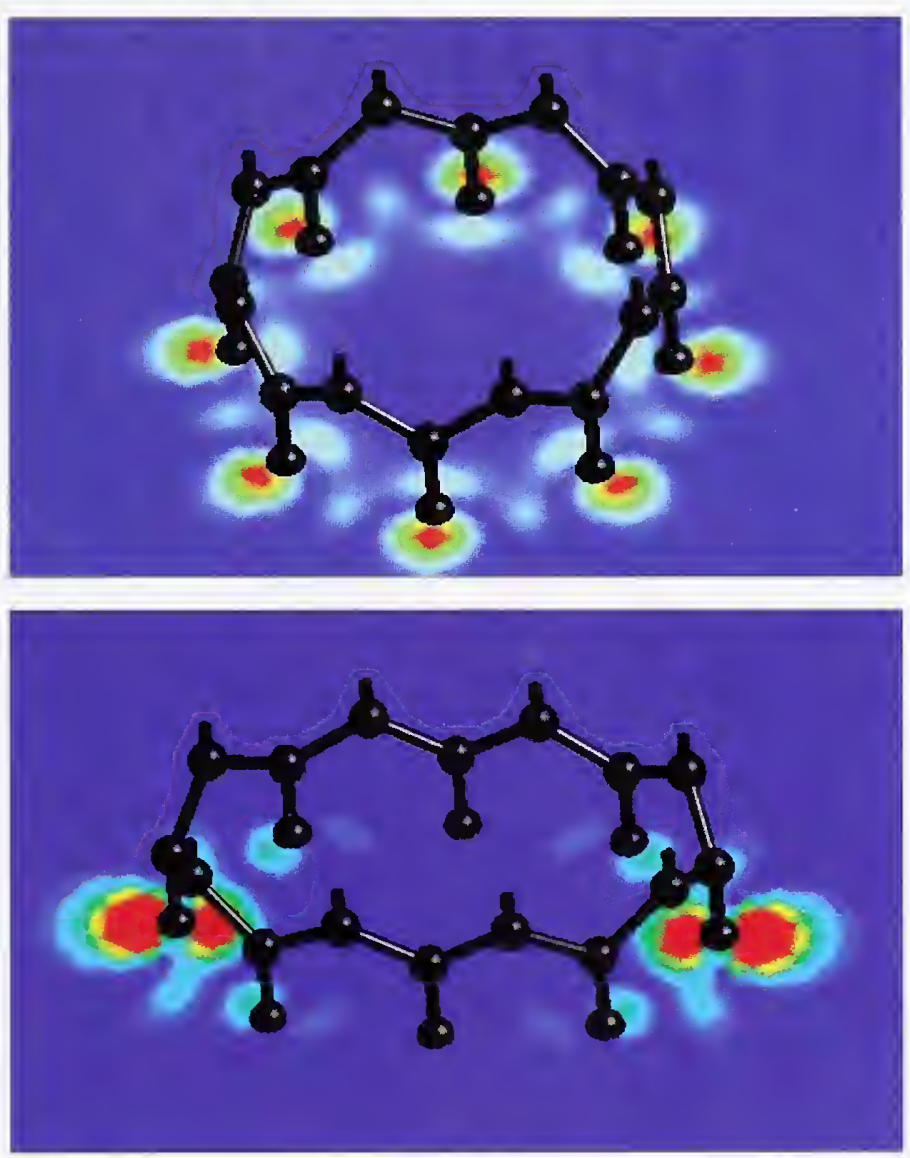

FIGURE 3. Contour plot of the first conduction band of an undeformed (top) and elliptically deformed (bottom) $(8,0)$ SWNT. See Ref. 1 for animation of this effect.

\section{References}

[1] See http://www.ncnr.nist.gov/staff/taner/nanotube.

[2] O. Gulseren, T. Yildirim, and S. Ciraci, Phys. Rev. Lett. 87, 116802 (2001).

[3] C. Kilic, S. Ciraci, O. Gulseren, and T. Yildirim, Phys. Rev. B62, R16345 (2000). 


\section{Serving the Scientific and Technological Communities}

$\mathrm{T}$ he role of the NCNR as a national user facility has expanded significantly over the past few years, as the last of the instruments envisioned in the original cold neutron project has become operational. The Disk-Chopper Spectrometer, the Filter-Analyzer Neutron Spectrometer, the High Flux Backscattering Spectrometer, and the Neutron Spin-Echo spectrometer now permit U.S. scientists to carry out neutron spectroscopy with greatly improved resolution and sensitivity. The thermal perfect-crystal small angle neutron scattering instrument (USANS) has expanded the length scale available by this technique to $10^{4} \mathrm{~nm}$. User experiments show a steadily increasing diversification in subject area and technique, enabled by the new instruments. We anticipate that the trend will continue over the next few proposal cycles.

User participation over the past 15 years shows continuing growth (see Fig. 1). The NCNR currently accommodates more than half of all neutron users in the U.S. While the Spallation Neutron Source is being built at Oak Ridge, the NCNR continues to be the nation's premier facility for providing neutrons to the U.S. research community.

\section{The NCNR User Program}

Researchers may obtain use of NCNR neutron beam instruments in several ways, the most direct being through the formal proposal system. Approximately twice a year, a

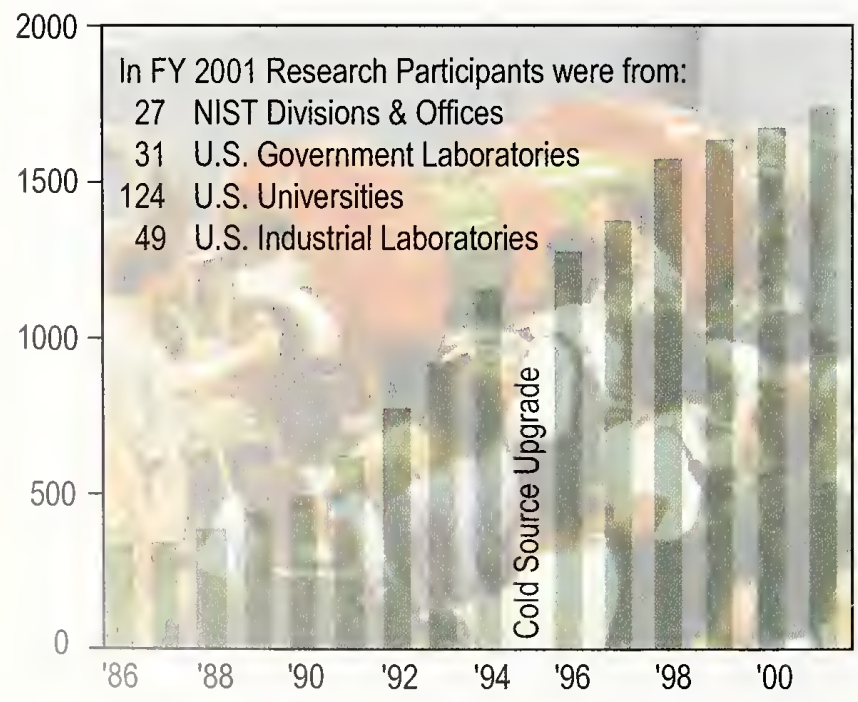

FGDPE 1. Research Participants at the NCNR.
Call for Proposals is issued. After a thorough review process by external referees and by the NCNR Program Advisory Committee (PAC), approved proposals are allocated beam time. The PAC is a panel of distinguished scientists with expertise across a broad range of neutron methods and scientific disciplines. It is the body primarily responsible for proposal review and recommending user policies for the NCNR, working closely with the Center's Director and staff. Its current membership includes Sanat Kumar (Penn State University, chair), Robert Briber (University of Maryland), Michael Crawford (DuPont), Dieter Schneider (Brookhaven National Laboratory), Kenneth Herwig (Oak Ridge National Laboratory), Yumi Ijiri (Oberlin College), Michael Kent (Sandia National Laboratories, John Tranquada (Brookhaven National Laboratory), and Andrew Allen (NIST).

At the recent PAC meeting in January 2001, the PAC considered 121 proposals for SANS and reflectometry, in addition to 49 for inelastic neutron scattering. Although we expect that both categories will see increased user demand in future proposal rounds, the latter area is likely to see more growth, since the new inelastic scattering spectrometers have added capabilities previously unavailable at U.S. neutron facilities.

\section{The Center for High Resolution Neutron Scattering}

The National Science Foundation (NSF) through the Center supports several NCNR instruments for High Resolution Neutron Scattering (CHRNS), a very important component of the user program. Until the present, CHRNS instruments included a $30 \mathrm{~m}$ SANS machine, the SPINS tripleaxis spectrometer, and USANS. Approximately $40 \%$ of the instrument time allocated by the PAC went to experiments carried out on CHRNS instruments. During the past year, the NSF approved a proposal to provide support for a substantial enlargement of CHRNS, enabling us to offer more beam time to users on the three new high-resolution inelastic scattering instruments. In the near future, another SANS diffractometer, the $8 \mathrm{~m}$ machine on neutron guide NG-1, which is presently used primarily for NIST programmatic research, will be upgraded to a more powerful $9 \mathrm{~m}$ 
instrument with a new detector, and made available to users through CHRNS. The expanded suite of CHRNS instruments will provide enhanced technical support and accessibility to the user community.

\section{Summer School on Methods and Applications of Neutron Spectroscopy}

The NCNR's seventh annual Summer School on neutron scattering, held from June 18-22, 2001, focused on high-resolution neutron spectroscopy. Most of the 32 participants were graduate and postdoctoral students; we also had an undergraduate student and a professor.

In a radical departure from the format of previous NCNR summer schools, plenary lectures were presented on the first morning only. After lunch the students were divided into four groups of eight, and each group performed an experiment using a different instrument (in one case a pair of instruments). Students learned the scientific motivation for the experiment, how the instrument works, and how to set up measurements. The instruments collected data overnight and on the following morning the data were analyzed. After lunch on each of the second, third and fourth days the groups rotated to new experiments. After lunch on the final day, eight of the students gave short presentations describing an experiment.

Students used the following instruments: the Spin Polarized Inelastic Neutron Spectrometer, the Disk Chopper Spectrometer, the High-Flux Backscattering Spectrometer, the Neutron Spin Echo spectrometer, and the Filter Analyzer Neutron Spectrometer. Together these instruments probe motions in materials over six decades in time (from approximately $10^{-7} \mathrm{~s}$ to $10^{-13} \mathrm{~s}$ ) and two orders of magnitude in distance $(0.1 \mathrm{~nm}$ to $10 \mathrm{~nm})$. Test samples included a geometrically frustrated antiferromagnet, a transition metal compound with organic ligands, a "symmetric top" molecule, and a system of spherical micelles. The experiments illustrated coherent scattering, incoherent scattering, magnetic scattering, and tunneling. In evaluating the school more than $80 \%$ of the participants described it as "excellent," and generally high ratings were received in all areas. The lectures and experiment handouts have been placed on the Web (www.ncnr.nist.gov/programs/

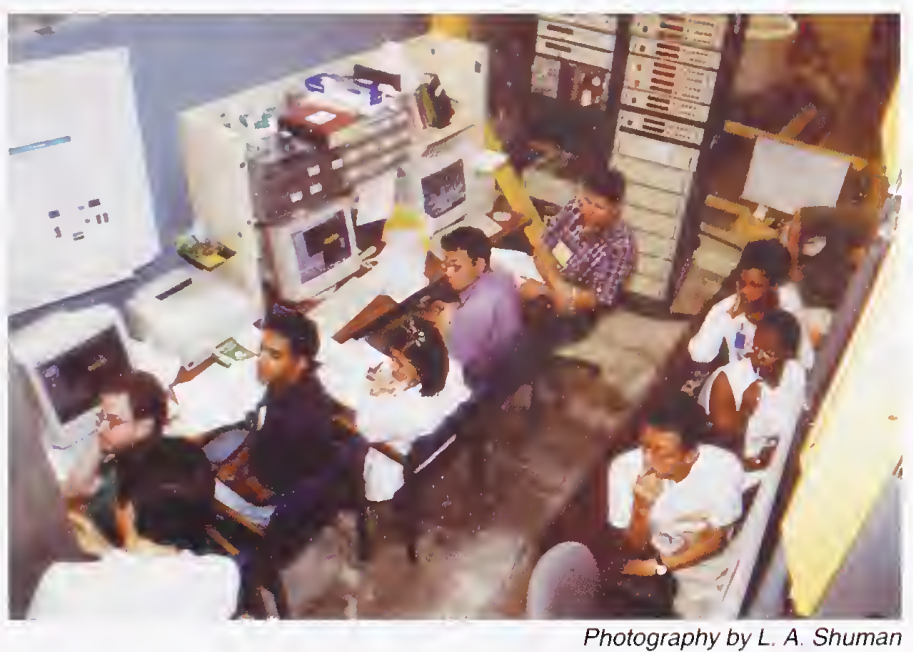

FIGURE 2. Seventh annual Summer School on Neutron Scattering participants analyze results with NCNR's Seung-Hun Lee (bottom left).

spectroscopy/SS01/SS01_materials.html.) A short report about the summer school will appear in "Neutron News." As in the past, the summer school was jointly sponsored with the National Science Foundation, which provided financial assistance to many of the university participants.

\section{Collaborations}

Direct collaborations on specific experiments remain a common way for users to pursue their ideas using NCNR facilities, accounting for approximately half of the number of instrument-days. The thermal-neutron triple-axis spectrometers are mainly scheduled in this way. Most of the time reserved for NIST on these and all other NCNR instruments is devoted to experiments that are collaborations with non-NIST users. Collaborative research involving external users and NIST scientists often produces results that could be not obtained otherwise.

Another mode of access to the NCNR is through Participating Research Teams (PRTs). In this case, groups of researchers from various institutions join forces to build and operate an instrument. Typically, $50 \%$ to $75 \%$ of the time on the instrument is then reserved for the PRT, and the remaining time is allocated to general user proposals. For example, a PRT involving ExxonMobil, the University of Minnesota, and NIST cooperates on the NG-7 $30 \mathrm{~m}$ SANS instrument. Similar arrangements involving other PRTs 
apply for the horizontal-sample reflectometer, the highresolution powder diffractometer, the filter-analyzer spectrometer, and the neutron spin-echo spectrometer.

\section{Independent Programs}

There are a number of programs of long standing located at the NCNR that involve other parts of NIST, universities, industrial laboratories, or other government agencies.

The Polymers Division of the Materials Science and Engineering Laboratory has two major program elements at the NCNR. In the first, the purpose is to help the U.S. microelectronics industry in addressing their most pressing materials measurement and standards issues. In today's integrated circuits and packages the feature size on a chip is ever shrinking, approaching $250 \mathrm{~nm}$, while the size of a polymer molecule is typically $5 \mathrm{~nm}$ to $10 \mathrm{~nm}$. As feature size shrinks, the structure and properties of interfaces play an increasingly important role in controlling the properties of the polymer layers used in interconnects and packages. NIST scientists use both neutron reflectivity and other neutron scattering methods to characterize polymer/metal interfaces with regard to local chain mobility, moisture absorption, glass transition temperature and crystalline structure.

In the second program element, the objective is to understand underlying principles of phase behavior and phase separation kinetics of polymer blends, both in the bulk and on surfaces, in order to help control morphology and structure during processing. SANS and reflectivity measurements in equilibrium, in transient conditions, and under external fields, provide essential information for general understanding as well as for specific application of polymer blend/alloy systems. Customers include material producers and users, ranging from chemical, rubber, tire, and automotive companies, to small molding and compounding companies. The focus of research on polymeric materials includes commodity, engineering and specialty plastic resins, elastomers, coatings, adhesives, films, foams, and fibers.

The ExxonMobil Research and Engineering Company is a member of the Participating Research Team (PRT) that operates, maintains, and conducts research at the NG-7
$30 \mathrm{~m}$ SANS instrument and the recently commissioned NG5 Neutron Spin Echo Spectrometer. The mission is to use those instruments, as well as other neutron scattering techniques, in activities that complement research at ExxonMobil's main laboratories as well as at laboratories of its affiliates around the world. The aim of these activities is to deepen understanding of the nature of ExxonMobil's products and processes, so as to improve customer service and to improve the return on shareholders' investment. Accordingly, and taking full advantage of the unique properties of neutrons, most of the experiments use SANS or other neutron techniques to study the structure and dynamics of hydrocarbon materials, especially in the fields of polymers, complex fluids, and petroleum mixtures. ExxonMobil regards its participation in the NCNR and collaborations with NIST and other PRT members not only as an excellent investment for the company, but also as a good way to contribute to the scientific health of the Nation.

The Nuclear Methods Group (Analytical Chemistry Division, Chemical Science and Technology Laboratory) has as its principal goals the development and application of nuclear analytical techniques for the determination of elemental compositions with greater accuracy, higher sensitivity and better selectivity. A high level of competence has been developed in both instrumental and radiochemical neutron activation analysis (INAA and RNAA). In addition, the group has pioneered the use of cold neutron beams as analytical probes with both prompt gamma activation analysis (PGAA) and neutron depth profiling (NDP). PGAA measures the total amount of a particular analyte present throughout a sample by the analysis of the prompt gamma rays emitted during neutron capture. NDP, on the other hand, determines concentrations of several important elements (isotopes) as a function of depth within the first few micrometers of a surface by energy analysis of the prompt charged particles emitted during neutron bombardment. These techniques (INAA, RNAA, PGAA, and NDP) provide a powerful combination of complementary tools to address a wide variety of analytical problems of great importance in science and technology, and are used to help certify a large number of NIST Standard Reference Materials. 
During the past several years, a large part of the Group's efforts has been directed toward the exploitation of the analytical applications of the guided cold-neutron beams available at the NIST Center for Neutron Research. The Group's involvement has been to design and construct stateof-the-art cold neutron instruments for both PGAA and NDP and provide facilities and measurements for outside users, while retaining and utilizing our existing expertise in INAA and RNAA.

The Center for Food Safety and Applied Nutrition, U.S. Food and Drug Administration (FDA), directs and maintains a neutron activation analysis (NAA) facility at the NCNR. This facility provides agency-wide analytical support for special investigations and applications research, complementing other analytical techniques used at FDA with instrumental, neutron-capture prompt-gamma, and radiochemical NAA procedures, radioisotope x-ray fluorescence spectrometry (RXRFS), and low-level gamma-ray detection. This combination of analytical techniques enables diverse multi-element and radiological information to be obtained for foods and related materials. The NAA facility supports agency quality assurance programs by developing in-house reference materials, by characterizing food-related reference materials with NIST and other agencies, and by verifying analyses for FDA's Total Diet Study Program. Other studies include the development of RXRFS methods for screening foodware for the presence of $\mathrm{Pb}, \mathrm{Cd}$ and other potentially toxic elements, use of instrumental NAA to investigate bromate residues in bread products, and use of prompt-gamma NAA to investigate boron nutrition and its relation to bone strength.

\section{The Neutron Interactions and Dosimetry Group}

(Physics Laboratory) provides measurement services, standards, and fundamental research in support of NIST's mission as it relates to neutron technology and neutron physics. The national and industrial interests served include scientific instrument calibration, electric power production, radiation protection, defense nuclear energy systems, radiation therapy, neutron radiography, and magnetic resonance imaging.

The Group's activities may be represented as three major activities. The first is Fundamental Neutron Physics including magnetic trapping of ultracold neutrons, operation of a neutron interferometry and optics facility, development of neutron spin filters based on laser polarization of ${ }^{3} \mathrm{He}$, measurement of the beta decay lifetime of the neutron, and investigations of other coupling constants and symmetries of the weak interaction. This project involves a large number of collaborators from universities and national laboratories.

The second is Standard Neutron Fields and Applications utilizing both thermal and fast neutron fields for materials dosimetry in nuclear reactor applications and for personnel dosimetry in radiation protection. These neutron fields include thermal neutron beams, "white" and monochromatic cold neutron beams, a thermal-neutron-induced ${ }^{235} \mathrm{U}$ fission neutron field, and ${ }^{252} \mathrm{Cf}$ fission neutron fields, both moderated and unmoderated. The third is Neutron Cross Section Standards including experimental advancement of the accuracy of neutron cross section standards, as well as evaluation, compilation and dissemination of these standards.

Several universities have also established long term programs at the NCNR. The University of Maryland is heavily involved in the use of the NCNR, and maintains several researchers at the facility. Johns Hopkins University participates in research programs in solid-state physics and in instrument development at the NCNR. The University of Pennsylvania is working to help develop biological applications of neutron scattering. It is also participating in the second stage construction of the filter analyzer neutron spectrometer, along with the University of California at Santa Barbara, DuPont, Hughes, and Allied Signal. The University of Minnesota participates in two PRTs, the NG-7 $30 \mathrm{~m}$ SANS and the NG-7 reflectometer. The University of Massachusetts also participates in the latter PRT. 


\section{Operations}

$\mathbf{T}$ he NIST neutron source (NBSR) operated for 240 full power (20 MW) days during the past year, or $100 \%$ of the scheduled time. Routinely, the NBSR is scheduled to operate a seven-week cycle, seven times a year. Each operating cycle includes 38 days on-line and 11 days shutdown for refueling, routine maintenance, and surveillance tests. (Figure 1 shows the operations staff adjusting the power-regulating rod.) This year, four days in March were required for maintenance on the Thermal Column Cooling System. The NBSR was shutdown on August 26, 2001. for a planned maintenance period of three months. During this time the cold source will be replaced, building transformers and switchgear will be replaced to provide additional electrical capacity, and the new cooling tower will undergo final hookup and testing. (Figure 2 shows the new cooling tower in the final stages of construction. For comparison the old cooling tower is seen on the left.)

A comparison has been made of thermal-hydraulic characteristics of the NBSR based on two calculations. The first calculation used correlations and simple models available when the licensed power level was raised to $20 \mathrm{MW}$ in 1985. The second used newer correlations that better represent research neutron source characteristics. The analysis shows that the heat transfer coefficient, onset of flow instability, and critical heat flux of the original calculation are all well within the operating conditions predicted by the new calculation. In addition, the NBSR also has an ample margin before the onset of nucleate boiling.

The above results are for extreme yet still conceivable conditions of operation. The Energy Sciences and Technology Department at the Brookhaven National Laboratory has been tasked to perform transient analysis with state-of-the art methods. Monte Carlo calculations will be used to accurately predict parameters that are used in the transient analysis.

Work is also continuing in the preparation of an updated license renewal submittal to the NRC. In conjunction with this effort, an in-service inspection and ultrasonic testing of the primary cooling system are planned for the summer/fall shutdown.

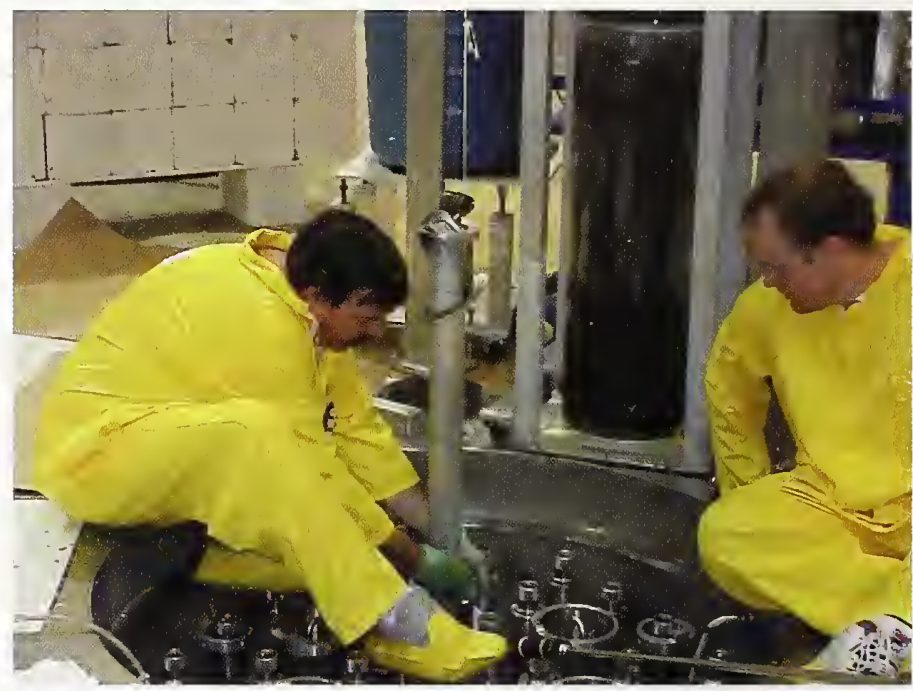

FIGURE 1. Bill Mueller and Greg Heller of the operations staff adjust the power-regulating rod.

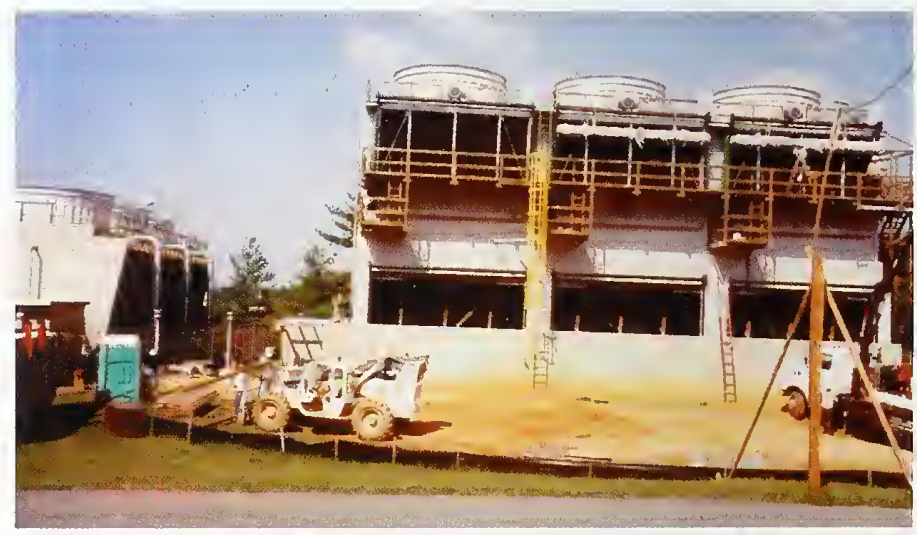

FIGURE 2. The new plume-abated cooling tower (right) nears completion. It will replace the lower capacity and less efficient system that had no abatement ability (left). 


\section{Instrumentation Developments}

\section{The Advanced Cold Neutron Source}

After six years of safe and reliable operation, the liquid hydrogen cold source cryostat at the NIST reactor is being replaced by one with a new advanced design. Based on extensive operating experience and sophisticated Monte Carlo calculations of the cold source and NIST reactor core, the new design improves the neutron coupling between the reactor fuel and moderator and the cold source cryostat. The advanced source, (called Unit 2), will differ from the original in many key respects, as can be seen in Fig. 1. The most important change is the addition of 60 liters of $\mathrm{D}_{2} \mathrm{O}$, partially surrounding the moderator chamber, reducing the
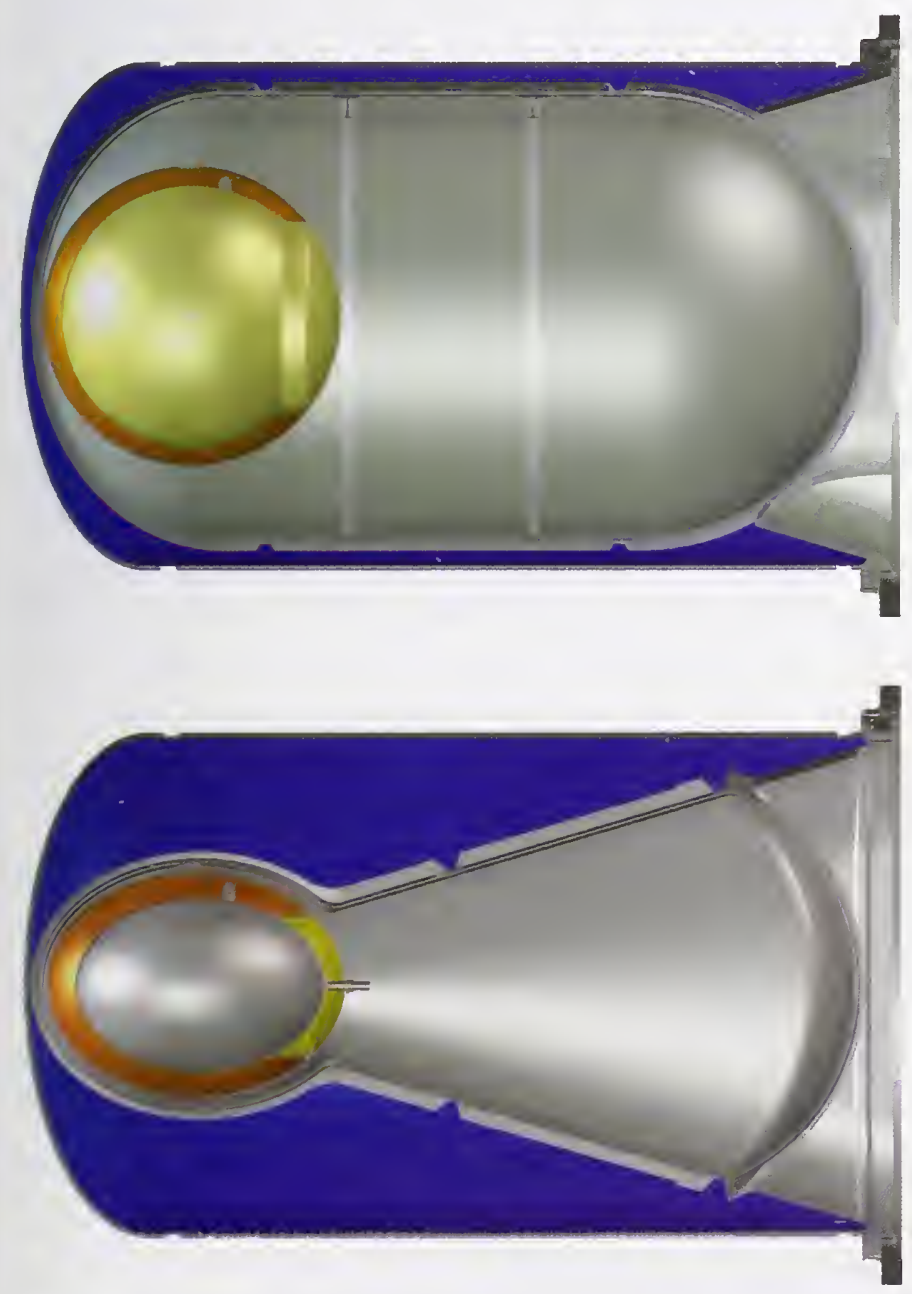

FIGURE 1. Comparison between the original (top) and new designs for the liquid hydrogen cold source cryostat at NIST. Heavy water volume is indicated in blue, liquid hydrogen in orange, and hydrogen vapor in yellow. moderator void volume and increasing the thermal neutron flux in the cryostat region by about $40 \%$.

The new cryostat is shaped like an ellipsoidal shell, rather than a spherical one. This results in a smaller volume so that more $\mathrm{D}_{2} \mathrm{O}$ can be introduced in the cryostat assembly. The center of the inner ellipsoid is offset, so the liquid hydrogen shell is $30 \mathrm{~mm}$ thick near the reactor core. This change results in an additional $5 \%$ to $10 \%$ increase in flux.

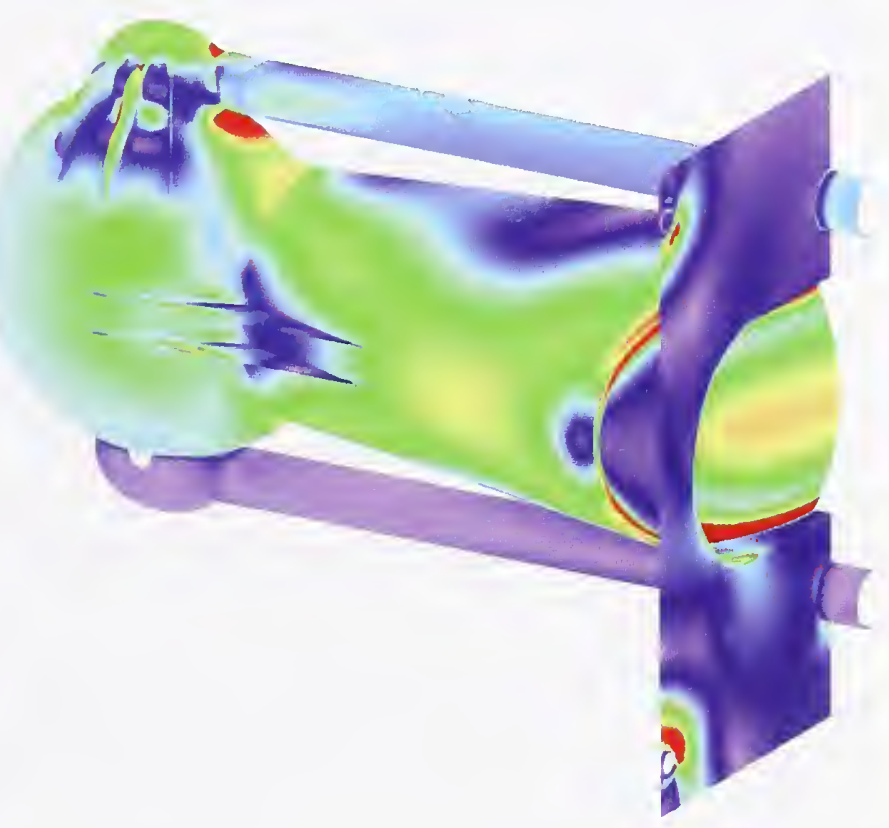

FIGURE 2. False color representation of stresses from finite element calculations of the helium containment jacket with an internal pressure of 82 atmospheres.

Finally, in contrast to the original design, the inner ellipsoid will be evacuated through a small port, removing most of the hydrogen vapor. This change will add another $15 \%$ to $20 \%$ gain. Based on these changes, users can expect an overall intensity increase of $50 \%$ to $80 \%$ between $2 \AA$ and $20 \AA$ with this design.

The complex geometry of the advanced source posed many engineering challenges. The moderator chamber is surrounded by a vacuum vessel, which is surrounded by a helium containment vessel, strong enough to withstand the design basis accidental detonation of liquid hydrogen and solid oxygen. These vessels determine the extent of the $\mathrm{D}_{2} \mathrm{O}$ 
volume, so they were designed to partially surround the moderator chamber, allowing an opening just large enough to fully illuminate the neutron guides. This "hour-glass" shape created regions of very high mechanical stress, analyzed and ultimately mitigated with the aid of finite element analysis (see Fig. 2). During the three-month period beginning at the end of August 2001, we are removing the old cryostat and installing Unit 2.

\section{Development of an Air-Pad and Floor System for New Neutron Spectrometers}

The NCNR is currently developing several new neutron triple axis spectrometers with a key design feature that allows interchangeability of the back-end detector/ analyzer systems. Exchanging the back-end system on these instruments requires a universal connection and positioning system between it and the sample position of the instrument. This interface must be capable of achieving an angular accuracy of 0.1 degrees, an angular precision of 0.01 degrees and a vertical positional accuracy of less than a millimeter.

The design approach adopted uses a commercial airpad system with a precision-leveled floor. After considering several different types of floors we chose to use epoxy as our

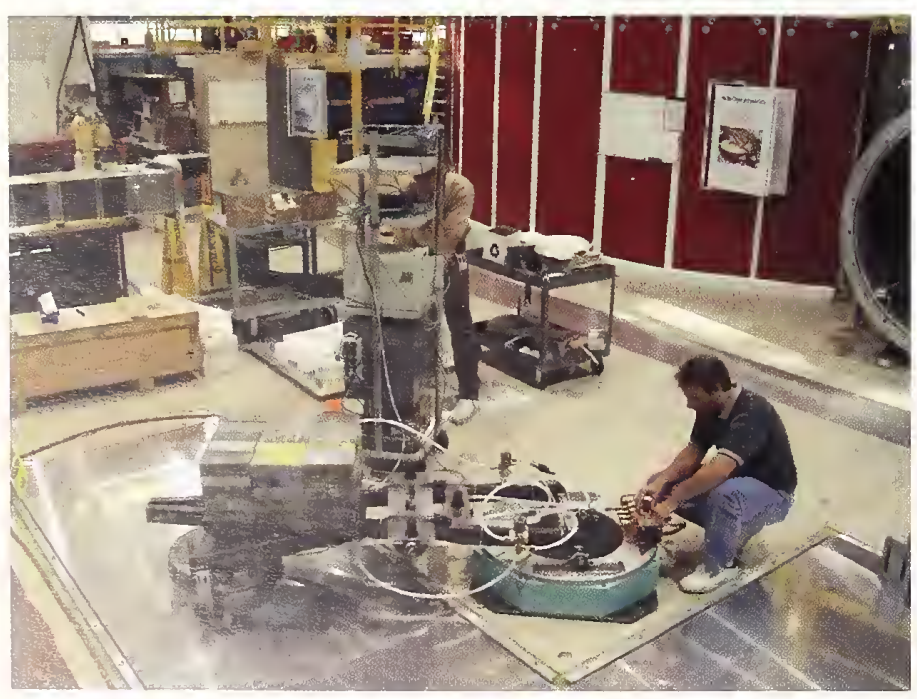

FIGURE 3. Michael Murbach and Colin Wrenn conduct tests on the prototype floor system for a triple axis spectrometer. sub-floor with precision cut and anodized aluminum plates as our contact surface. Epoxy will self-level, fill in holes, and mask over any imperfections in the concrete floor and results in a highly smooth and level surface to build on. The epoxy is also low cost, relatively easy to use, and provides great flexibility for repair or maintenance. The aluminum plates provide protection to the epoxy from cryogenic spills, increased wear resistance, and an easily removable/reusable floor.

We have developed and tested a prototype single airpad system that has successfully demonstrated the construction techniques for the floor system and the ability of the air pad positioning system to meet the accuracy and repeatability requirements for a 1 ton test load. Currently, a three-pad prototype system is under development that will allow us to test control system concepts needed in a final design.

\section{Development of an Intercalated Graphite Monochromator}

As part of an ongoing collaboration between NIST and Harvard University to measure the neutron lifetime using magnetically trapped ultracold neutrons (UCNs), a new monochromator has been developed. In the lifetime experiment, ultracold neutrons (UCN: neutrons with energies $\approx 100 \mathrm{neV}$ ) are produced in a superfluid ${ }^{4} \mathrm{He}$ bath inside the trapping apparatus using the "superthermal process." A beam of cold neutrons is introduced into the helium, in which neutrons with wavelength near $0.89 \mathrm{~nm}$ scatter to UCN energies by creation of a single phonon. Only neutrons within a narrow wavelength band $( \pm 0.01 \mathrm{~nm}$ for our current magnetic trap) participate in this single-phonon production mechanism. Since the wavelength band for UCN production occupies only a small fraction of the neutron spectrum produced by the cold source (see Fig. 4), a significant signal-to-background improvement is possible with appropriate wavelength filtering of neutrons that enter the apparatus. Bragg reflection from a crystalline monochromator with a lattice-plane spacing $d$ can be used to select a narrow wavelength band for neutrons with wavelengths up to $2 d$. Unfortunately, widely used materials for neutron monochromatization, such as pyrolytic graphite, have insufficiently large $d$ spacing for reflecting $0.89 \mathrm{~nm}$ neutrons. One attractive class of materials for producing 
long wavelength monochromators is intercalated graphite. Intercalation is the process of inserting foreign atoms or molecules between the layers of the graphite, and results in a crystal with a larger $d$ spacing. Intercalated graphites are formed in "stages," where the stage number defines the number of planes of graphite between layers of intercalant atoms.

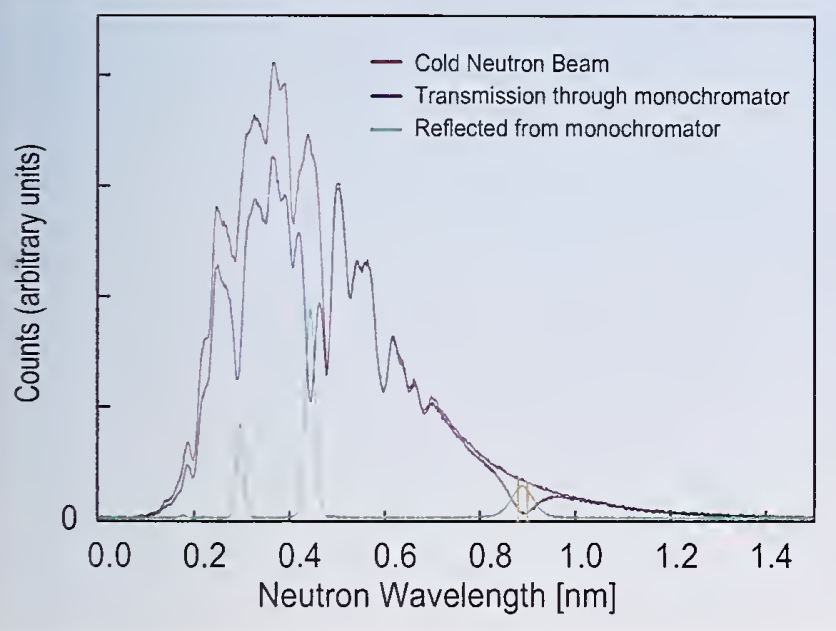

FIGURE 4. Neutron wavelength spectra were measured using a time-offlight technique. Only $1 \%$ of the neutrons emerging from guide NG-6 (red) lie within the wavelength band (between the orange vertical bars) available for production of UCN. The spectra of neutrons transmitted (blue) and reflected (green) by the monochromator are also shown.

We have designed, fabricated, and tested a new $0.89 \mathrm{~nm}$ monochromator using stage-2 potassium-intercalated graphite. Nine pieces of intercalated graphite are tiled to form the monochromator, which has a total size of $6 \mathrm{~cm} \times 15 \mathrm{~cm}$. Heating potassium and graphite to two different temperatures in an evacuated glass cell produces each tile. Performance of the assembled monochromator was determined using time-of-flight techniques (see Fig. 4). The monochromator reflects more than $80 \%$ of the neutrons incident upon it in the wavelength band relevant for UCN production. However, only $3 \%$ of all incident neutrons are reflected. When combined with additional techniques that filter out neutrons from higher order Bragg reflections, the monochromator results in an improvement of the ratio of useful to total neutrons of almost two orders of magnitude while sacrificing few trapped neutrons. During the summer of 2001, this new monochromator was used in conjunction with the trapping apparatus in a temporary setup. Presently, we are permanently installing the monochromator at the fundamental physics station at NG-6. This installation will place the monochromator much closer to the end of the neutron guide and will result in an increase by an additional factor of two in the number of trapped UCNs.

\section{NCNR Computer and Software Environment}

The NCNR's computerized data acquisition and reduction environment encompasses all stages of research: experiment preparation, data collection and analysis of results. NCNR provides site-specific tools that augment the tools in researchers' own scientific arsenal. Some specific areas of recent activity are described below:

- NCNR is providing simulation tools that model the behavior of our instruments, to evaluate the feasibility of proposed experiments. They can be used by prospective users, especially those who aren't already familiar with neutron scattering experiments, to explore "what-if" scenarios with different sample and instrument configurations. Currently, we have simulations of small angle scattering experiments, with reflectivity and stress measurement simulations being developed.

- Researchers who plan to use NCNR instrumentation must submit their proposals for review. We have created a web-based proposal submission system that tracks user proposals from submission to review and ranking, to the final instrument time allocation.

- All instruments available at NCNR are computercontrolled. The control software running on these computers must satisfy two seemingly contradictory needs: simplicity, for straightforward use, and sophistication, for power uses. New, more complex instruments, such as the spin echo spectrometer, require carefully designed graphical user interfaces (GUIs) that hide unnecessary detail, while still providing access to all functions, if needed. Since the NCNR instruments run around the clock, the data acquisition functions must be capable of automation and unattended operation. To this end, the experiment control software is scriptable, in addition to the GUI interface. The scriptable, command-based interface fulfills "power-user" needs, while 


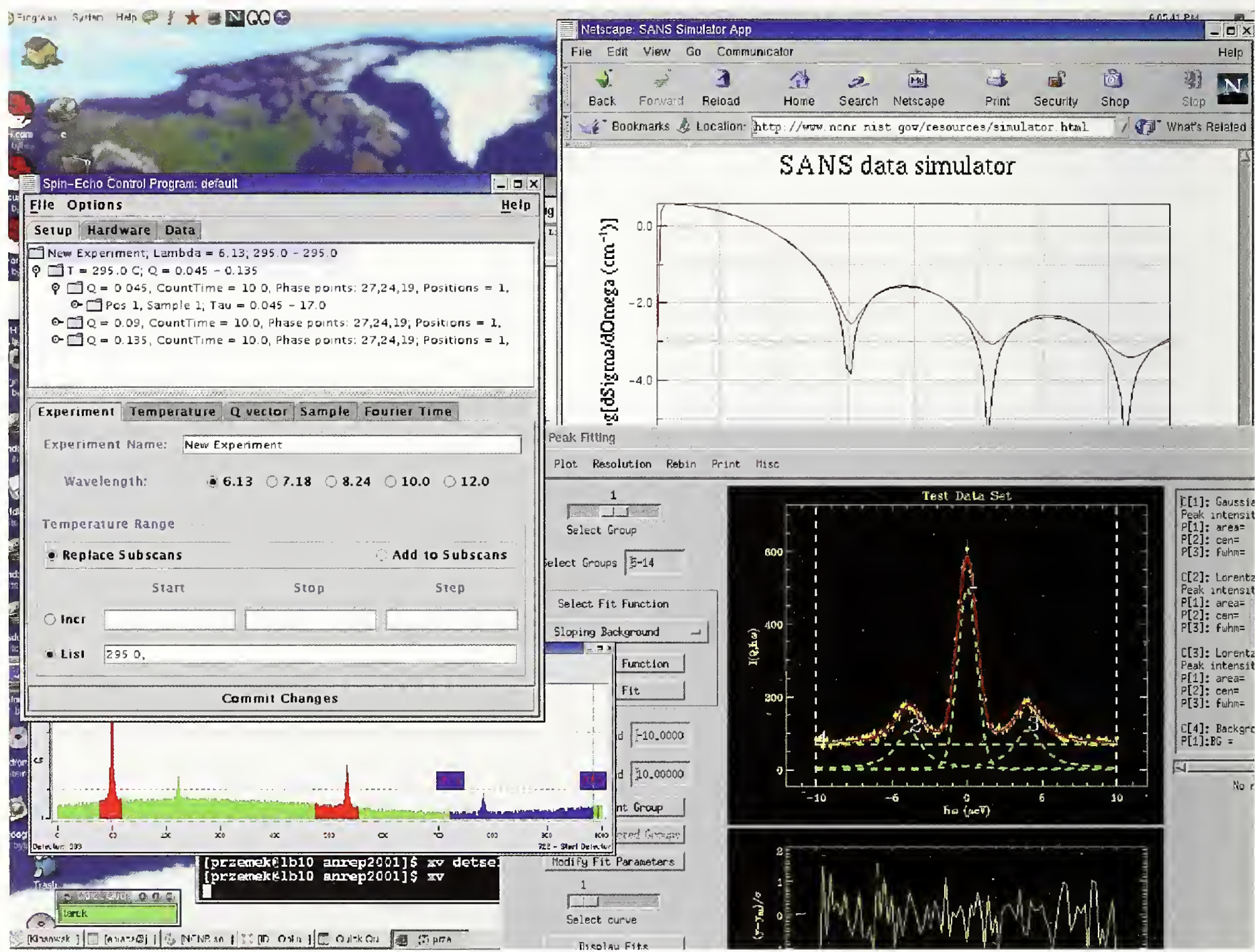

FIGURE 5. A screenshot of several NCNR applications. Clockwise from top left corner: the web-based simulator of a SANS experiment, peak analysis application, Time-of-flight detector selection, and a Spin Echo instrument control panel.

a simplified GUI interface tends to be easier to learn and use.

- Rapid post-acquisition data reduction is important to guide the ongoing experiments, and, later, to aid in the processing of data to obtain the scientific results. Many data reduction procedures are common to all the instruments. The Data Visualization and Analysis Environment (DAVE) is a set of tools commonly used by NCNR scientific community; these tools include programs to manipulate and compare data, extract parameters of theoretical models by data fitting, etc.
- NCNR maintains a sophisticated computer infrastructure including a modern network installation, and an ensemble of nearly 400 computers and other IT equipment, for scientific and office computing, ranging from email servers to supercomputer-style parallel computing clusters.

- NCNR participates in inter-laboratory collaborations developing software for specialized domains. Perhaps the best known of these is the GSAS software for crystallographic data reduction. Another ongoing NCNR collaboration is in international scattering data exchange format, NeXuS 


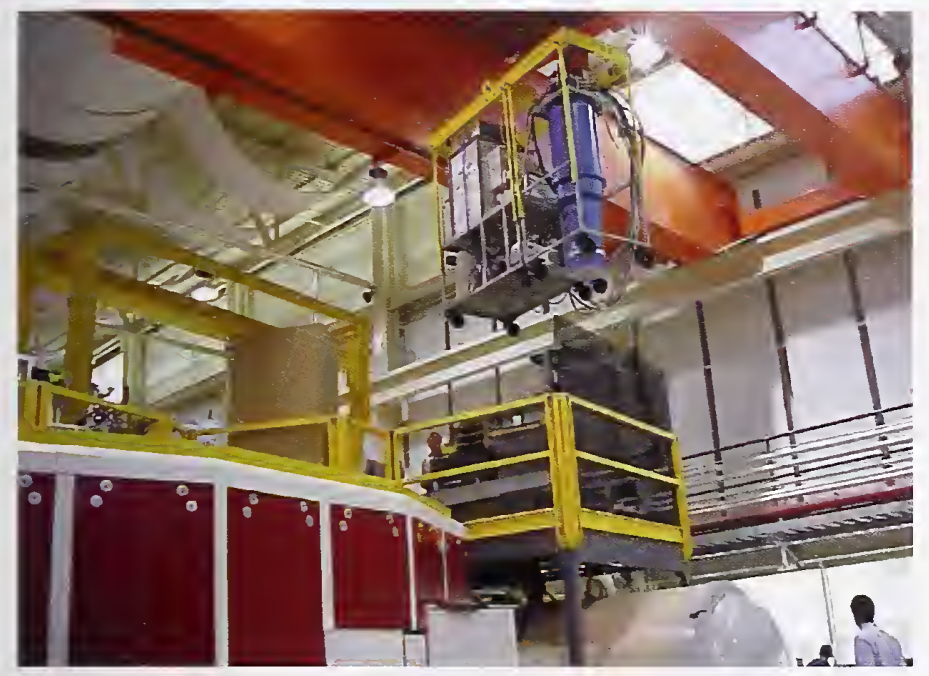

FIGURE 6. Superconducting magnet/dilution refrigerator as it is being lifted onto the DCS spectrometer. The adjustable lifting frame compensates for the lifting point shifting in case liquid cryogens are added.

\section{Sample Environment Team}

The sample environment team expanded by two new members this year, bringing the total number to four employees that are actively working to ensure that the needs of researchers working at the NCNR are met. This team works to improve the infrastructure sample environment equipment and to support neutrons scattering instruments at the NCNR. A principal objective is to provide safe and easy to use equipment to the research staff. Equipment development activities are done in collaboration with the engineering and technical staff, with input from both staff and guest scientists. As an example, a new superconducting magnet/ dilution refrigerator system needed modifications for use on the Disk Chopper Spectrometer (DCS) for an experiment. The impracticality of separating the cryostat from its support pumps and electronics led to the decision to lift the entire system onto the instrument for use. A new adjustable lifting frame was design and manufactured to lift the various components of the magnet (see Fig. 6).
Another experiment required the development of a system capable of going to temperatures as high as $675 \mathrm{~K}$ with a high voltage applied to the sample. The design goal was to be able to achieve a $5 \mathrm{kV}$ potential difference across the sample. After continued development work, we were able to apply a potential difference of $8 \mathrm{kV}$ to the sample while at the maximum temperature of $675 \mathrm{~K}$. In addition to the human safety features designed in from the beginning, a circuit was developed which prevents the continued arcing that can occur if the sample breaks down during the course of the experiment. 
Abdurashitov, J. N., Gavrin, V. N., Kalikhov, A.V., Matushko, V. L., Shikhin, A. A., Yants, V. E., Zaborskaia, O. S., Adams, J. M., Nico, J. S., Thompson, A. K., "A High Resolution Low Background Fast Neutron Spectrometer," in Nuclear Instruments and Methods, in press.

Adams, C. P., Lee, Y. S., Lee, S.-H., Lynn, J. W., "Triple-Axis Research at NIST," Neutron News 12 (2), 15 (2001).

Adams, C. P., Lynn, J. W., Mukovskii, Y. M., Arsenov, A. A., Shulyatev, D. A., "Charge Ordering and Polaron Formation in the Magnetoresistive Oxide $\mathrm{La}_{0.7} \mathrm{Ca}_{0.3} \mathrm{MnO}_{3}$," Phys. Rev. Lett. 85 (18), 3954 (2000).

Adams, J. M., "Results From the NIST Round Robin Test of Fissionable Dosimeters in a Reactor Leakage Spectrum," REACTOR DOSIMETRY, in Proceedings Radiation Metrology and Assessment, ASTM STP 1398, edited by J. Williams, D. Vehar, F. Ruddy, D. Gilliam, (ASTM, West Conshohocken, PA, Feb. 2001), p. 295.

Adams, J. M., Greenhouse, L. A., "Nuclear Science at the National Institute of Standards and Technology: A Historical Overview," Transactions of the American Nuclear Society, November 2000 , (Washington, DC), 83, 492 (2000).

Akpalu, Y. A, Karim, A., Satija, S., Balsara, N. P., "Suppression of Lateral Phase Separation in Thin Polyolefin Blend Films," Macromol. 34 (6), 1720 (2001).

Allen, A. J., Ilavsky, J., Long, G. G., Wallace, J. S., Berndt, C. C., Herman, H., "Microstructural Characterization of YttriaStabilized Zirconia Plasma-Sprayed Deposits Using Multiple Small-Angle Neutron Scattering," Acta Mater. 49 (9), 1661 (2001).

Allen. A. J., Long, G. G., Boukari, H., Ilavsky, J., Kulkarni, A., Sampath, S., Herman, H., Goland, A. N., "Microstructural Characterization Studies to Relate the Properties of Thermal Spray Coating to Feedstock and Spray Conditions," Surf. Coat. Techn., in press.

Allman, B. E., McMahon, P. J., Nugent, K. A., Paganin. D., Jacobson, D. L., Arif, M., Werner, S. A., "Phase Radiography with Neutrons," Nature 408, 158 (2000).

Amos, T. G., Sleight, A. W., "Negative Thermal Expansion in Orthorhombic NbOPO „" J. Solid State Chem., 160, 230 (2001).

Anderson, C. R., Andersen, K. H., Stirling, W. G., Sokol, P. E., Dimeo, R. M., "Dynamics of Superfluid ${ }^{4} \mathrm{He}$ Confined in Xerogel Glass," Phys. Rev. B, in press.

Anderson, D. L., Capar, S. G., "Identification of Lead in Metal Food Can Seanı Using ${ }^{57}$ Co RXRFS," J. Radioanal. Nucl. Chem. 249 (1), 77 (2001).

Anderson, D. L., Cunningham, W. C., "Revalidation and Long-Term Stability of National Institute of Standards and Technology Standard Reference Materials," J. AOAC International, 83 (5), $1121(2000)$.
Anderson, D. L., Cunningham, W. C., Capar, S. G., Baratta, E. J., Mackill, P., "Nuclear Methods for Food Analysis at the U. S. Food and Drug Administration," J. Radioanal. Nucl. Chem. 249 (1). 29 (2001).

Bao, W., Aeppli, G., Lynn, J. W., Pagliuso, P. G., Sarrao, J. L., Hundley, M. F., Thompson, J. D., Fisk, Z., “Anisotropic ThreeDimensional Magnetic Fluctuations in Heavy Fermion CeRhIn ${ }_{5}$," Phys. Rev. Lett., in press.

Bao, W., Christianson, A. D., Pagliuso, P. G., Sarrao, J. L., Thompson, J. D., Lacerda, A. H., Lynn, J. W., "Effect of La-Doping on Magnetic Structure in Heavy Fermion CeRhIn ${ }_{5}$," Physica B, in press.

Bao, W., Pagliuso, P. G., Sarrao, J. L., Thompson, J. D., Fisk, Z., Lynn, J. W., "Magnetic Structure of Heavy Fermion $\mathrm{Ce}_{2} \mathrm{RhIn}_{8}$," Phys. Rev. B64 (2), 0401 (2001).

Bao, W., Pagliuso, P. G., Sarrao, J. L., Thompson, J. D., Fisk, Z., Lynn, J. W., Erwin, R. W., "Incommensurate Magnetic Structure of CeRhIn ," Phys. Rev. B63 (21), 9901 (2001).

Bateman, F. B., Boukharouba, N., Brient, C. E., Carlson, A. D., Grimes, S. M., Haight, R. C., Massey, T. N., Wasson, O. A., "New Measurements of the H(n,n)H Angular Distribution," REACTOR DOSIMETRY, Radiation Metrology and Assessment, ASTM STP 1398, edited by J. Williams, D. Vehar, F. Ruddy, D. Gilliam, (American Society for Testing Materials, West Conshohocken, PA, Feb. 2001), p. 431.

Bauer, B. J., Amis, E. J., "Characterization of Dendritically Branched Polymers by SANS, SAXS, and TEM," Tomalis/Frechet: Dendritic Polymers. 2000, in press.

Bauer, B. J., Lin, E. K., Lee, H. J., Wang, H., Wu, W.-L., "Structure and Property Characterization of Low-k Dielectric Porous Thin Films," J. Electron. Mater. 30 (4), 304 (2001).

Becker, P. R., Mackey, E. A., Demiralp, R., Epstein. M. S., Donais, M. K., Goessler, W. A., Porter, B. J., Wise, S. A., "Heavy Metals and Other Trace Elements Measured in Tissues of Marine Mammals Archived by the Alaska Marine Mammal Tissue Archival Project from the Bering Sea and Cook Inlet, Alaska," in Proceedings of The AMAP Heavy Metals Workshop, in press.

Becker, P. R., Pugh, P. R., Schantz, M. M., Mackey, E. A., Oflaz, R. D., Epstein, M. S., Donais, M. K., Porter, B. J., Wise, S. A.. Mahoney, B. A., "Persistent Chlorinated Compounds and Elements in Tissues of Cook Inlet Beluga Whales Banked by the Alaska Marine Mammal Tissue Archival Project," NISTIR 6702, (Gaithersburg, MD), p. 67.

Baklanov, M. R., Kondoh, E., Lin, E. K., Gidley, D. W., Lee, H.-J., Mogilnikov, K. P., Sun, J. N., "Comparative Study of Porous SOG Films with Different Non-Destructive Instrumentation," in Proceedings of the National Interconnect Technology Conference, in press.

Bellitto, C., DiMarco, M. G., Branford, W. R., Green, M. A., Neumann, D. A., "Cation Distribution in Ga-Doped $\mathrm{Li}_{1.02} \mathrm{Mn}_{2} \mathrm{O}_{4}$ ", Solid State Ionics 140 (1), 77 (2001). 
Bianco-Peled, H., Dori, Y., Satija, S., Schneider, J., Sung, L., Tirrell, M., "Structural Study of Langmuir Monolayers Containing Lipidated Poly (Ethylene Glycol) and Peptides," Langmuir, in press.

Blackman, M. J., Redford, S., "Northern Syrian Luster and Fruitware: Petrographic and Chemical Implications for Productions and Distribution," Antiquity, in press.

Bode, P., de Nadai Fernandes, E. A., Greenberg, R., "INAA as a Primary Method in Metrology for Chemical Measurements," in Proceedings Metrolsul. '99, 2nd South American Congress of Metrology (Foz do Iguacu, Brazil, 2000), p. 89.

Borchers, J. A., Ijiri, Y., Lind, D. M., Ivanov, P. G., Erwin, R. W., Qasba, A., Lee, S.-H., O`Donovan, K. V., Dender, D. C., "Detection of Field-Dependent Antiferromagnetic Domains in Exchange-Biased $\mathrm{Fe}_{3} \mathrm{O}_{4} / \mathrm{NiO}$ Superlattices," Appl. Phys. Lett. 77 (25), 4187 (2000).

Bordallo, H. N., Chapon, L. C., Cook., J. C., Copley, J. R. D., Goremychkin, E., Kern, S., Lee, S.-H., Yildirim, T., Manson, J. L., "Spin Excitations in 3D Molecular Magnets Probed by Neutron Scattering," Appl. Phys. A, in press.

Borgoul-Maciejczyk, P., Ondov, J. M., "Heterogenous Nature of Metallic Components in Baltimore Particulate Matter," Environ. Sci. Technol., in press.

Bossev, B. P., Kline, S. R., lsraelachvilli, J. N., Paulaitis, M. E., "Pressure-Induced Freezing of the Hydrophobic Core Leads to a L1AEH1 Phase Transition for $\mathrm{C}_{12} \mathrm{E}_{5}$ Micelles in $\mathrm{D}_{2} 0$," Langmuir, in press.

Bossev, D. P., Ferdinand, S., Lesemann, M., Paulaitis, M. E., "The Effect of Kilobar Pressures on Microstructure in $\mathrm{C}_{12} \mathrm{E}_{5}$ Nonionic Microemulsions," Langmuir, in press.

Bossev, D. P., Ferdinand, S., Paulaitis, M. E., "Effect of Pressure on Microstructure in Water-in-Oil and Oil-in-Water Microemulsions for $\mathrm{C}_{12} \mathrm{E}_{5} \mathrm{n}$-Octane/ $\mathrm{D}_{2} 0$ Mixtures," Langmuir, in press.

Bossev, D., Kline, S., Paulaitis, M. E., "Pressure-Induced Aggregation of $C_{12} E_{5} D_{20}$ Wormlike Micelles," Langmuir, in press.

Bourdarot, F., Lynn, J. W., Baggio-Saitovitch, E., Huang, Q., Sanchez, D. R., Skanthakumar, S., "Magnetic Behavior of the Rare Earth Compounds RNiBC by Neutron Scattering," Phys. Rev. B64, 104410 (2001).

Brome, C. R., Butterworth, J. S., Dzhosyuk, S. N., Mattoni, C. E. H., McKinsey, D. N., Doyle, J. M., Huffman, P. R., Dewey, M. S., Wietfeldt, F. E., Golub, R., Lamoreaux, S. K., Coakley, K. J., "Magnetic Trapping of Ultracold Neutrons," Phys. Rev. C 63 (5), 5502 (2001).

Brown, A. D. B., Rennie, A. R., "Monodisperse Colloidal Plates Under Shear," Phys. Rev. E 62 (1), 851 (2000).

Bu, Z., Chowdhuri, Z., Callaway, D. J. E., "Coexistence of Fluctuating Secondary Structure and Long-Range Tertiary Interaction in Highly Denatured Alpha-Lactalbumin - A Neutron Scattering Study," Protein Sci., in press.
Bu, Z., Cook, J., Callaway, D. J. E., "Dynamics Regimes and Correlated Structural Dynamics in Native and Denatured AlphaLactalbumin," J. Mol. Biol., in press.

Buckley, C. E., Birnbaum, H. K., Lin, J. S., Spooner, S., Bellmann, D., Staron, P., Udovic, T. J., Hollar, E., "Characterization of H Defects in the Aluminium-Hydrogen System Using Small-Angle Scattering Techniques," J. Appl. Crystallogr. 34, 119 (2001).

Burauer, S., Belkoura, L., Strey, R., Co, C. C., Kaler, E. W., "Size and Polydispersity of Polymer Particles Obtained by Cryo-TEM, SANS and DLS," Elektronenmikroscopie 21, 20 (2001).

Burgess, 1., Zamlynny, V., Szymanski, G., Lipkowski, J., Majewski, J., Smith, G., Satija, S., lvkov, R., "Electrochemical and Neutron Reflectivity Characterization of Dodecyl Sulfate Adsorption and Aggregation at the Gold-Water Interface," Langmuir 17, 3355 (2001).

Butler, P. D., Porcar, L., Warr, G. G., Hamilton, W. A., comment on "Evidence of a Sponge-to-Lamellar Phase Transition Under Shear by X-Ray Scattering Experiments in Couette Cell," Phys. Rev. Lett., in press.

Caffrey, P. F., Ondov, J. M., "Dry Deposition of Elemental Aerosol Constituents to Lake Michigan Surface Waters, by Size and by Source," Environ. Sci. Technol., in press.

Caffrey, P. F.. Ondov, J. M., "Dry Removal of Elemental Aerosol Particulate Constituents in Near Shore Gradient of Southern Lake Michigan," Environ. Sci. Tech., in press.

Caliskan, G., Kisliuk, A., Tsai, A. M., Soles, C. L., Sokolov, A. P., "Protein Dynamics in Viscous Solvents," J. Biophys., in press.

Cambell, B. J., Billings, S., Lynn, J. W., Sinha, S. K., "The Structure of Jahn-Teller Polarons in the Colossal Magnetoresistive Manganites," in press.

Campbell, B. J., Osborn, R., Argyriou, D. N., Vasiliu-Doloc, L., Mitchell, J. F., Sinha, S. K., Ruett, L. U., 1slam, Z., Lynn, J. W., "The Structure of Nanoscale Polaron Correlations in a Colossal Magnetoresistive Manganite," Phys. Rev. B, in press.

Cappelletti, R. L., "The National Institute of Standards and Technology Center for Neutron Research (NCNR)," Neutron News, 12 (2), 10 (2001).

Cappelletti, R. L., Clutter, L. K., edited by, "NIST Center for Neutron Research Accomplishments and Opportunities, NCNR 2000," N1ST SP 962 (2001).

Cappelletti, R. L., Clutter, L. K., edited by, "N1ST Center for Neutron Research, Programs and Accomplishments," N1ST1R 6598.

Cappelletti, R. L., Glinka, C. J., Krueger, S., Lindstrom, R. A., Lynn, J. W., Prask, H. J., Prince, E., Rush, J. J., Rowe, J. M., Satija, S. K., Toby, B. H., Tsai, A., Udovic, T. J., "Materials Research With Neutrons at N1ST,” J. Res. Natl. Inst. Stand. 106 (1), 187 (2001).

Carpenter, J. M., Iverson, E. B., Mildner, D. F. R., "Time Focusing of Pulsed-Source Crystal Analyzer Spectrometers II Practical Expressions," Nucl. lnstrum. Meth. A, in press. 
Cava, R. J., Yamaura, K., Loureiro, S., Huang, Q., Erwin, R. W., Lynn, J. W., Young, D., "Magnets, Mischief and Metals in Cobalt Analogs of the Superconducting Cuprates," Physica C 341, 351 (2000).

Chen, S. H., Liao, C.. Fratini, E., Baglioni, P., Mallamace, F., "Interaction, Critical, Percolation and Kinetic Glass Transitions in Pluronic L-64 Micellar Solutions," Colloid Surface, 183, 95 (2001).

Chen, W. C., Heuser, B. J., King, J. S., "SANS Investigation of Deuteride (Hydride) Precipitation and Decomposition Morphology in Single Crystal Pd," J. Appl. Crystallogr. 33, 442 (2000).

Chen, Y. Y., Honda, Z., Zheludev, A., Broholm, C., Katsumata, K., Shapiro, S. M., "Field-Induced Three- and Two-Dimensional Freezing in a Quantum Spin Liquid," Phys. Rev. Lett. 86 (8), $1618(2001)$.

Chen, Y. Y., Lodge, T. P., Bates, F. S., "Entropically Driven Phase Separation of Highly Branched Linear Polyolefin Blends," J. Polym. Sci. Pol. Phys. 22, 2965 (2000).

Cheng, Y. T., Mildner, D. F. R., "A Sensitive High Resolution Neutron Imaging Detector," Nucl. Instrum. Meth. A 454 (2), 452 (2000).

Cheng, Y. T., Mildner, D. F. R., Chen-Mayer, H. H., Sharov, V. A., Glinka, C., "An lmaging Plate Detector for Small-Angle Neutron Scattering," J. Appl. Crystallogr. 33, 1253 (2000).

Chen-Mayer, H. H., Lamaze, G. P., Mildner, D. F. R., Zeissler, R., Gibson, W. M., "Neutron Imaging and Prompt Gamma Activation Analysis Using a Monolithic Capillary Neutron Lens," in Proceedings of the 3rd International Congress on Analytic Sciences, Analytical Science, in press.

Chen-Mayer, H. H., Lamaze, G. P., Satija, S. K., "Characterization of BPSG Films Using Neutron Depth Profiling and Neutron/X-Ray Reflectometry," in Proceedings of 2000 International Conference on Characterization and Metrology for ULSI Technology, edited by D. G. Seiler, A. C. Diebold, W. M. Bullies, T. J. Shaffner, R. McDonald, E. J. Walters (AIP Press, Woodbury, NY), 550, 407 (2001)

Choi, S. M., Barker, J. G., Glinka, C. J., Cheong, Y. T., Gammel, P. L., "Focusing Cold Neutrons with Multiple Biconcave Lenses for Small-Angle Neutron Scattering," J. Appl. Crystallogr. 33 (792), 2000 .

Choi, S. M., Briber, R. M., Bauer, B. J., Liu, D.-W., Gauthier, M., "Small Angle Neutron Scattering of Blends of Arborescent Polystyrenes," Macromol. 33 (17), 6495 (2000).

Choi, S. M., Lynn, J. W., Lopez, D., Gammel, P. L., Varma, C. M.. "Direct Observation of Spontaneous Weak-Ferromagnetism in the Superconductor ErNi, B C," Phys. Rev. Lett. 86, 7121 (2001)

Christianson, R. J., Leheny, R. L., Birgeneau, R. J., Erwin, R. W., "Critical Dynamics of a Spin-5/2 Two-Dimensional Isotropic Antiferromagnet," Phys. Rev. B63 (14), 0401 (2001).

Co, C. C., De Vries, R., Kaler E. W., "Small-Angle Neutron Scattering Study of Monomer Partitioning," Macromol. 34, 3224 (2001).

Cohen, R. E., Gulseren, O., "Thermal Equation of State of Tantalum," Phys. Rev. B63, 224101 (2001).

Copley, J. R. D., "NIST Recommended Practice Guide; The Fundamentals of Neutron Powder Diffraction," NIST SP 960-2, in press.
Cosgrove, T., Howe, A., Thomsen, L., Armes, S. P., Billingham, N., "The Structure of Hydrophilic-Hydrophobic Block Copolymers and Their Interactions With Salt and a Surfactant," Langmuir, in press.

DeVries, R., Co, C. C., Kaler, E. W., "Influence of Monomer Partitioning, Termination, and Diffusion Limitations on Polymerization Kinetics," Macromol. 34, 3233 (2001).

DeWall, J., Dimeo, R. M., Sokol, P. E., "Diffusion and Phase Transitions of Molecular Hydrogen in Zeolites," Phys. Rev. B, in press.

Dewey, M. S., Kessler, E. G., "Precision Measurement of Fundamental Constants Using GAMS4,” J. Res. Natl. Inst. Stan. 105 (1), 11 (2000).

Dimeo, R. M., Chowdhuri, Z., Meyer, A., Gehring, P. M., Neumann, D. A., "The NIST High Flux Backscattering Spectrometer," Appl. Phys. A, in press.

Dimeo, R. M., Neumann, D. A., "Low Temperature Dynamics of Confined Methyl Iodide," Phys. Rev. B63 (1), 4301 (2001).

Dimeo, R. M., Neumann, D. A., "The Effects of Confinement on Rotational Tunneling," Appl. Phys. A, in press.

DiNoia, T. P., Kirby, C. F., Van Zanten, J. H., McHugh, M. A., "SANS Study of Polymer-Supercritical Fluid Solutions: Transitions from Liquid to Supercritical Fluid Solvent Quality," J. Am. Chem. Soc. 33 (17), 6321 (2000).

Divita, F., Terry, J., Kidwell, C. A., Ondov, J. M., Pandis, S., "Characterization of Primary Aerosol Emissions from a Municipal Incinerator Via Ground-Level Sampling with a Micro-Orifice 1mpactor." Atmos. Environ., in press.

Douglas, J. F., Kent, M. S., Satija, S. K., Karim, A., "Swelling of Grafted Polymer 'Brushes,' " in Proceedings Encyclopedia of Materials: Science and Teclinology, in press.

Downing, R. G., Iyengar, G. V., "Methodological Issues in the Analytical Determination of Boron," Environ. Health Perspect., in press.

Dubsky, J., Prask, H. J., Matejicek, J., Gnäupel-Herold, T., "Stresses in Plasma Sprayed $\mathrm{Cr}_{2} \mathrm{O}_{3}$ Coatings Measured by Neutron Diffraction," in Proceedings of the International Conference on Neutron Scattering (Munich, Germany, September 2001), in press.

Eid, K., Portner, D., Borchers, J. A., Loloee, R., Tsai, M., O’Donovan, K. V., Pratt, W. P., Bass, J., "Mean-Free Path Effects in CPP Magnetoresistance of Magnetic Multilayers," Phys. Rev B, in press.

Elliot, J., Meuse, V., Silin, V., Krueger, S., Woodward, J. T., PetralliMallow, T. P., Plant, A. L., "Biomedical Membranes on Metal Supports: Opportunities and Challenges," in Proceedings of the Biomolecular Films: Design, Function, and Application, edited by J. Rusling (New York, Marcel Dekker, Inc.), in press.

Esker, A. R., Grull, H., Satija, S. K., Han, C. C., "Probing Polymer Dynamics with Ultra-Thin Membranes by Neutron Reflectivity" Polymer Preprints 221, (2001).

Esker, A. R., Grull, H., Wegner, G., Satija, S. K., Han, C. C., "Isotopic Selectivity in Ultra Thin Langmuir-Blodgett Membranes of a Cross-Linked Cellulose Derivative," Langmuir 17, 4688 (2001).

Evmenenko, G., Bauer, B. J., Kleppinger, R., Forier, B., Dehaven, W., Amis, E. J., Miscehnko, N., Reynaers, H., "The Influence of Molecular Architecture and Solvent Type on the Size of Structure of Poly (Benzyl Ether) Dendrimers by SANS," Chem. Phys. 202 (6), 891 (2001). 
Farrow, R. F. C., Marks, R. F., Toney, M. F., Hannibal, K., David, S., Kellock, A., Borchers, J. A., O'Donovan, K. V., Smith, D. J., "Spontaneous Chemical Ordering and Exchange Bias in Epitaxial $\mathrm{Mn}_{0.52} \mathrm{Pd}_{0.48} / \mathrm{Fe}(001)$ Bilayers Prepared at Room Temperature," Appl. Phys. Lett., in press.

Feeney, M., Brown, C. M., Tsai, A., Neumann, D. A., Debenedetti, P. G., "Incoherent Quasi-Elastic Neutron Scattering From Fructose-Water Solutions," J. Phys. Chem. 105 (32), 7799 (2001).

Ferdinand, S., Lesemann. M., Paulaitis, M. E., "Effect of Pressure on Microstructure of $\mathrm{C}_{12} \mathrm{E}_{5}$ n-Octane-in- $\mathrm{D}_{2} 0$ Microemulsions," Langmuir, 16, 10106 (2000).

FitzGerald, S. A., Thomas, J. J., Neumann, D. A., Livingston, R. A., "A Neutron Scattering Study of the Role of Diffusion in the Hydration of Tricalcium Silicate," Cement and Concrete Res., in press.

Fratini, E., Chen, S.-H. Baglioni, P., Cook, J., Copley, J., "Dynamic Scaling of Quasi-Elastic Neutron Scattering Spectra From Interfacial Water," Phys. Rev. E, in press.

Freeman, K. S., Beck Tan, N. C., Trevino, S. F., Kline, S., McGown, L. B., Kiserow, D. J., "Size and Polydispersity Determinations of AOT/Bile Salt Reversed Micelles Obtained by Small-Angle Neutron Scattering," Langmuir 17 (13), 3912 (2001).

Fultz, B., Witham, C. K., Udovic, T. J., "Distributions of Hydrogen and Strains in $\mathrm{LaNi}_{5}$ and $\mathrm{LaNi}_{4.75} \mathrm{Sn}_{0.25}$," J. Alloys Compnds., in press.

Garvey, C. J., Parker, I. H., Simon, G. P., Whittaker, A. K., Knott, R. B., "An Experimental Study by NMR and SANS of the Ambient Hydration of Paper," in Proceedings of the 12th Fundamental Research Symposinm, (Pulp and Paper Fundamental Research Society, Oxford), in press.

Gehring, P. M., Park, S.-E., Shirane, G., "Dynamical Effects of the Nanometer-Sized Polarized Domains in $\mathrm{Pb}\left(\mathrm{Zn}_{1 / 3} \mathrm{Nb}_{2 / 3}\right) \mathrm{O}_{3}$ " Phys. Rev. B63 (22), 4109 (2001).

Geissler, E., Horkay F., Hecht, A. M., "'Structure of Polymer Solutions and Gels Containing Fillers," Macromol. Symp. 171, 171 (2001).

Geissler, E., Hecht, A. M., "Treatment of Incoherent Signal Subtraction" in Proceedings of the Frontiers in SAXS and SANS, ESRFILL-CEA Workshop (Grenoble, France, February 12-13, 1999), in press.

Geissler, E., Horkay, F., Hecht, A. M., Deschamps, P., "Neutron Scattering From Elastomer Gels," Kautschuk, Gummi, and Kunstoffe, in press.

Geissler, E., Horkay, F., McKenna, G. B., Deschamp, P., "Osmotic and Mechanical Observations of Swollen Polyisoprene Rubber and its Microscopic Structure," in Proceedings of Worldwide Amazon Rubber Conference (Manaus, Brazil, October 31 - November 4, 1999), in press.

Gentile, T. R., Jones, G. L., Thompson, A. K., Rizi, R. R., Dimitrov, I. E., Roberts, D., Gefter, W., Schnall, M. D., Leigh, J. S., “A Compact Compressor for Application of Metastability-Exchange Optical Pumping of ${ }^{3} \mathrm{He}$ to Human Lung Imaging," Magn. Reson. Med., in press.
Gentile, T. R., Rich, D. R., Thompson, A. K., Bailey, C., Gericke, M., Hussey, D., Neff, B., Snow, W. M., Jones, G. L., Wildman, E., "The NIST-Indiana-Hamilton Polarized ${ }^{3} \mathrm{He}$ Spin Filter Program," in Proceedings of the Fundamental Physics with Pulsed Nentron Beans Conference, edited by C. R. Gould, G. L. Green, F. Plasil, W. M. Snow (World Scientific, Singapore, 2001), p. 230.

Gibson, W. M., Chen-Mayer, H. H., Mildner, D. F. R., Prask, H. J., Schultz, A. J., Youngman, R., Gnäupel-Herold, T., Miller, M. E., Vitt, R., "Polycapillary Optics Based Neutron Focusing for Small Sample Neutron Crystallography," in Proceedings of the Adv. In X-Ray Analysis 45, in press.

Giebultowicz, T. M., Kepa, H., Blinowski, J., Kacman, P., "Neutron Scattering Studies of Interlayer Correlations in Magnetic Semiconductor Superlattices," Physics E, in press.

Gilra, N., Cohen C., Briber, R. M., Bauer, B. J., Hedden, R., Panagiotopoulos, A. Z., "A SANS Study in Strained and Unstrained End-Linked Elastomers," Macromol., 34 (22), 7773 (2001).

Gilliam, D. M., "NIST Neutron Physics and Dosimetry," in Proceedings of the Transactions of the American Nuclear Society, November 12-16, 2000, (Washington, DC, 2000), p. 496.

Glagolenko, I. Y., Carney, K. P., Kern, S., Goremychkin, E. A., Udovic, T. J., Copley, J. R. D., Cook, J. C., "Quantitative Analysis of $\mathrm{UH}_{3}$ in $\mathrm{U}$ Metal and $\mathrm{UO}_{2}$ Matrices by Neutron Vibrational Spectroscopy," Appl. Phys. A, in press.

Glinka, C. J., "Review of Methods of X-Ray and Neutron Scattering in Polymer Science," by Ryong-Joon Roe, Physics Today 54 (3), 60 (2001).

Gnäupel-Herold, T., "Single Crystal Elastic Constants From Powder Measurements," in Proceedings of Accuracy in Powder Diffraction. III (NIST Special Publication), in press.

Gnäupel-Herold, T., Haeffner, D. R., Prask, H. J., Matejicek, J., "Stress Determination by Whole Pattern Analysis-Application to Synchrotron HEXRD Measurements on Thermal Sprayed Coatings," in Proceedings of The Sixth International Conference on Residnal Stresses, (IOM, London, 2000), p. 751.

Gnäupel-Herold, T., Matejicek, J., Prask, H. J., "Mechanical Properties of Plasma Sprayed Coatings - Measured by Diffraction," in Proceedings of the 9th International Metallurgical Conference Metal 2000, (Ostrava, CZ), paper no. 508 (CD-ROM).

Gnäupel-Herold, T., Prask, H., Fisher, J., Cheng, X., "Residual Stress Modification by Means of Ultrasonic Impact Treatment," in Proceedings of the Annual Conference of Society for Experimental Mechanics. (Portland, OR, June 2001), in press.

Gnäupel-Herold, T., Prask, H. J., Magiera, J., "Effect of Grinding Strategy on Accumulation of Damage in Rails: Neutron Diffraction Investigation of Residual Stresses in Transverse and Oblique Cut Rail Slices," in Proceedings of The Sixth International Conference on Residnal Stresses (IOM, London, 2000). p. 879 .

Green, M. A., Waldron, J. E. L., Neumann, D. A., "Charge and Spin Ordering in Monoclinic $\mathrm{Nb}_{12} \mathrm{O}_{29}$," J. Am. Chem. Soc. 123 (24), 5833 (2001).

Greenberg, C. C., Foster, M. D., Turner, C. M., Corona-Galvan, S., Cloutet, E., Quirk, R. P., Butler, P. D., Hawker, C., "Effective Interaction Parameter Between Branched and Linear PS," J. Polym. Sci. 39, 2549 (2001). 
Greenberg, C. C., Teale, D. M., Foster, M. D., Turner, C. M., CoronaGalvan, S., Cloutet, E., Quirk, R. P., Majkrzak, C. F., Demaree, D., "Surface Segregation in Blends of Linear and Regularly Star Branched Polystyrene," Macromol., in press.

Greenberg, C. C., Foster, M. D., Turner, S., Corona-Galva, E., Cloutet, R., Quirk, P., Butler, P. D., Hammouda, B., Hawker, C., "Effective Interaction Parameter Between Branched and Linear PS," J. Polym. Sci. 39 (21), 2549 (2001).

Grohn, F., Bauer, B. J., Akpalu, Y. A., Jackson, C. L., Amis, E. J., "Dendrimers as Templates for the Formation of Inorganic Nanoclusters," Macromol. 33 (16), 6042 (2000).

Grohn, F., Bauer, B. J., Amis, E. J., "Organic-Inorganic Hybrid Particles by Dendrimer Nanotemplating," in Proceedings of the Materials Research Society Meeting (April 2000), p. 62.8.

Gruell, H., Sung, L., Karim, A., Douglas, J. F., Satija, S. K., Hayashi, M., Jinnai, H., Hashimoto, T., Han, C. C., "Surface Segregation of a Symmetrical Segregating Polymer Blend Film Above and Below the Critical Point of Phase Separation," Phys. Rev. Lett., in press.

Gulseren, O., Yildirim, T., Ciraci, S., "Tunable Adsorption on Carbon Nanotubes," Phys. Rev. Lett. 87 (11), 116802 (2001).

Gulseren, O., Yildirim, T., Ciraci, S., Kilic, C., "First-Principles Study of the Electronic Structure of Carbon Nanotubes: Variable and Reversible Band Gap Engineering," Phys. Rev. B, in press.

Guo, L., Colby, R. H., Lin, M. Y., Dado, G. P., "Micellar Structure Changes in Aqueous Mixtures of Nonionic Surfactants," J. Rheol. 45, 1223 (2001).

Hagemann, I. S., Huang, Q., Gao, X. P. A., Ramirez, A. P., Cava, R. J., "Geometric Magnetic Frustration in $\mathrm{Ba}_{2} \mathrm{Sn}_{2} \mathrm{ZnGa}_{4} \mathrm{Cr}_{6} \mathrm{O}_{22}$ : A TwoDimensional Spinel Based Kagome Lattice," Phys. Rev. Lett. 86 (5). 894 (2001)

Hammouda, B., "SANS From Polymers Under Pressure," Neutron News 12, 20 (2001).

Han, C. C.. Hobbie, E. K., Nakatani, A. I., Karim, A., "Critical Temperature Shift and Mixing in Polymer Blends Under Simple Shear Flow," J. Phys. Soc. Japan., 70, 317 (2001).

Han, C. C.. Schmidt, G., Nakatani. A. I., Butler, P., “A Small Angle Neutron Scattering Study on Polymer Clay Solutions" in Proceedings ACS PMSE, (September 2001, Chicago, IL) p. 219.

Han, C. C., Schmidt, G., Nakatani, A. I., Karim, A., "Characterization of Polymer-Clay Solutions by Rheology, Flow Birefringence and SANS," Polymer Prints, 42, 269 (2001).

Hanakahi, L. A., Bu, Z., Maizels, N., "The C-Terminal Domain of Nucleolin Accelerates Nucleic Acid Annealing," Biochem. 39. 15493 (2000)

Hayashi, M., Hashimoto, T., Hasegawa, H., Takenaka, M., Gruell, H., Esker, A. R., Weber, M., Satija, S. K., Han, C. C., Nagao, M., "Interface Between a Polysulfone and Polyamide as Studied by Combined Neutron Reflectivity and Small-Angle Neutron Scattering Techniques," Macromol. 33 (22), 8375 (2000).

Hayashi, M., Hashimoto, T., Weber, M., Gruell, H., Esker, A., Han, C. C., Satija, S. K., "Transient Interface Instability in Bilayer Polymer Films as Observed by Neutron Reflectivity Studies," Macromol., in press.

He, T., Huang, Q., Cava, R. J., "Comparison of the Magnetic Properties of Isoelectronic $\mathrm{Sr}_{x}\left(\mathrm{Na}_{0.5} \mathrm{La}_{0.5}\right)_{1-\mathrm{x}} \mathrm{RuO}_{3}$ and $\mathrm{Sr}_{x} \mathrm{Ca}_{1-\mathrm{x}} \mathrm{RuO}_{3}$," Phys. Rev. B63, 4402 (2001).
He, T., Huang, Q., Ramirez, A. P., Wang, Y., Regan, K. A., Rogado, N., Hayward, M. A., Haas, M. K., Slusky, J. S., Inumaru, K., Zandbergen, H. W., Ong, N. P., Cava, R. J., "Superconductivity in the Non-Oxide Perovskite $\mathrm{MgCNi}_{3}$ ", Nature 411, 54 (2001).

Heath, C. H., Streletzky, K. A., Wolk, J., Wyslouzil, B. E., Strey, R., " $\mathrm{D}_{2} \mathrm{O}-\mathrm{H}_{2} \mathrm{O}$ Condensation in Supersonic Nozzles Experiments," in Proceedings Nucleation and Atmospheric Aerosols, 2000, edited by B. H., Hale, Kulmama, M., (American Institute of Physics, New York, 2000), p. 59.

Hecht, A. M., Horkay, F., Geissler, E., "Neutron Scattering Investigations on a Bimodal Polymer Gel," J. Phys. Chem. B 105, 5637 (2001).

Hecht, A. M., Horkay, F., Geissler, E., "Structure of PDMS Solutions Containing Fumed Silica," Phys. Rev. E., in press.

Heller-Zeisler, S. F., Borgoul, P. V., Moore, R. R., Smoliar, M., Suarez, A. E., Ondov, J. M., "Comparison of INAA and RNAA Methods with Thermal-Ionization Mass Spectrometry for Iridium Determinations in Atmospheric Tracer Studies," J. Radioanyl. Nuc. Chem. 244, 93 (2000).

Heuser B. J., Chen, W. C., "Small-Angle Neutron Scattering Measurements of Deuteride (Hydride) Formation and Decomposition in Single-Crystal Pd," Phys. Rev. B, in press.

Hiraka, H., Endoh, Y., Fujita, M., Lee, Y. S., Kulda, J., Ivanov, A., Birgeneau, R. J., "Spin Fluctuations in the Underdoped High-TC Cuprate $\mathrm{La}_{1.93} \mathrm{Sr}_{0.07} \mathrm{CuO}_{4}$," J. Phys. Soc. Japan 70 (3), 853 (2001).

Ho, D. L., Briber, R. M., Glinka, C. J., "Studies on Organically Modified Clays Using Scattering Techniques," ACS Symposium of Polymer Nanocomposites 804, (11) (2001).

Ho, D. L., Briber, R. M., Glinka, C. J., "Characterization of Organically Modified Clays Using Scattering and Microscopy Techniques," Chem. Mater. 13 (5), 1923 (2001).

Hoffman, A., Fitzsimmons, M. R., Dura, J. A., Majkrzak, C. F., "Investigating Magnetic Proximity Effects in NiO/Pd with Polarized Neutron Reflectometry," Phys. Rev. B, in press.

Hone, J. H. E., Howe, A. M., Cosgrove, T., "A Rheological and Small Angle Neutron Scattering Study of the Structure of Gelatin/ Polyelectrolyte Complexes Under Shear," Macromol. 33 (4), 1199 (2000).

Horkay, F., Basser, P. J., Hecht, A. M., Geissler, E., "Osmotic and SANS Observations on Sodium Polyacrylate Hydrogels in Physiological Salt Solutions," Macromol. 33, 8329 (2000).

Horkay, F., Basser, P. J., Hecht, A. M., Geissler, E., "Ion-Exchange Induced Change in the Structure and Osmotic Properties of Sodium Polyacrylate Hydrogels," Macromol. Symp. 171, 201 (2001).

Horkay, F., Hecht, A. M., Basser, P. J., Geissler, E., "Comparison Between Natural Gels and Neutralized Polyelectrolyte Gels in the Presence of Divalent Cations," Macromol. 34, 4285 (2001).

Horkay, F., Hecht, A. M., Geissler, E., "Neutron Scattering Investigations on Filled Polymer Gels," in Proceedings of the 219th ACS National Meeting 41 (San Francisco, CA, March 26-30, 2000) 219, 158 (2000).

Horkay, F., Hecht, A. M., Geissler, E., "Neutron Scattering Investigations on Bimodal Polymer Gels," J. Phys. Chem. B 105, 5637 (2001).

Horkay, F., McKenna, G. B., Deschamps, P., Geissler, E., "Neutron Scattering Properties of Randomly Cross-Linked Polyisoprene Gels," Macromol. 33 (14), 5215 (2000). 
Horkay, F., McKenna, G. B., Deschamps. P., Geissler, E., "Relationship Between Microscopic Structure and Osmotic and Mechanical Behavior of Polyisoprene Gels," Macromol. 33, 5215 (2000).

Horkay, F., McKenna, G. B., Geissler, E., "Nanostructures in Swollen Polymer Networks," in Proceedings of the Polymer Networks 98, 14th Polymer Networks, Group Conference (Trondheim,

Norway, June 28 - July 1, 1998), The Wiley Polymer Networks Group Review, in press.

Huang, Q., He, T., Regan, K. A., Rogado, N., Hayward, M., Haas, M. K., Inumaru, K., Cava, R. J., "Temperature Dependence of the Structural Parameters of the Non-Oxide Perovskite Superconductor $\mathrm{MgCNi}_{3}$," Physica $\mathrm{C}$, in press.

Hudson. B. S., "Inelastic Neutron Scattering: A Tool in Molecular Vibrational Spectroscopy and a Test of Ab Initio Methods," J. Phys Chem. A 105, 3949 (2001).

Huffman, P. R., Brome, C. R., Butterworth, J. S., Coakley, K. J,, Dewey. M. S., Dzhosyuk. S. N.. Gilliam, D. M., Golub, R., Greene, G. L., Habicht, K., Lamoreaux, S. K., Mattoni, C. E. H., McKinsey, D. N., Wietfeldt, F. E., Doyle. J. M., "Progress Towards Magnetic Trapping of Ultra-Cold Neutrons," Nucl. Instrum. Meth. A 440 (3), 522 (2000).

Huffman, P. R., Brome, C. R., Butterworth, J. S., Coakley, K .J., Dewey, M. S., Dzhosyuk, S. N., Golub, R., Greene, G. L., Habicht, K., Lamoreaux, S. K., Matton, C. E. H., McKinsey, D. N., Wietfeldt, F. E., Doyle, J. M., "Magnetic Trapping of Neutrons," Nature 403 (6765), 62 (2000).

Huffman, P. R., Brome, C. R., Butterworth, J. S., Dzhosyuk, S. N., Golub, R.. Lamoreaux, S. K., Mattoni, C. E. H., McKinsey, D. N., Doyle, J. M., "Magnetically Stabilized Luminescent Excitations in Hexagonal Boron Nitride." Lumin. 92 (4), 291 (2001).

Huffman, P. R., Coakley, K. J., Thompson, A. K., Yang, G. L., Wietfeldt, F. E., Alvine, K. J., Brome, C. R., Dzhosyuk, S. N., Mattoni, C. E. H., Michniak, R. A., McKinsey, D. N., Yang, L., Doyle, J. M., Golub, R., Habicht, K., Koch, J., Lamoreaux, S. K., Leite, M. L., Maia, L., Rodrigues, A. C., Zanotto, E. D., "Magnetic Trapping of Ultracold Neutrons: Prospects for an Improved Measurement of the Neutron Lifetime," in Proceedings of The Workshop on Fundamental Plysics with Pulsed Neutron Beams (World Scientific Publishing Co. Pte. Ltd., Singapore, 2001), p.156.

Hwang, S.-R., Li, W.-H., Lee, K. C., Lynn, J. W., Luo, H. M., Ku, H. C. "Structural and Magnetic Properties of $\operatorname{Pr}_{1+x} \mathrm{Ba}_{2-x} \mathrm{Cu}_{3} \mathrm{O}_{7+y}$ " Phys. Rev. B63 (17), 2401 (2001).

Hwang, S.-R., Li, W.-H., Lee, K. C., Lynn, J. W., Wu, C.-G., "Spiral Magnetic Structure of Fe in van der Waals Gapped FeOCI," Phys. Rev. B62 (21), 14157 (2000).

Ilavsky, J., Stalick, J. K., Wallace, J., in Proceedings of the Ist International Thermal Spray Conference (May 8-11, 2000, Montreal, Quebec Canada) edited by C. C. Berndt (New York), p. 1185.

Ivkov, R., Butler, P. D., Satija, S. K., "Effect of Solvent Flow on a Polymer Brush: A Neutron Reflectivity Study of the Brush Height and Chain Density Profile," Langmuir 17 (10), 2999 (2001).
Ivkov, R., Gehring, P. M., Maliszewskyj, N. C., Krishnamoorti, R., "Structure and Dynamics of Polymers in Confined Geometries: Neutron Studies of Intercalated Clay-Polymer Nanocomposites," in Proceedings SPE 57th Annual Technical Conference and Exhibits, (Society of Plastics Engineers, Inc., Brookfield, 2000), p. 1783 .

James, W. D., Zeisler, R., "Uptake of Oxygen in a Coal Standard Reference Material Determined by 14-MeV Neutron Activation Analysis," J. Radioanal. Nucl. Ch. 248 (1), 233 (2001).

Jeng, U., Lin, T.-L., Wang, L. Y., Chiang, L. Y., Ho, D. L., Han, C. C., "SANS Structural Characterization of Fullerenol-Derived Star Polymers in Organic Solutions," Appl. Phys. A, in press.

Jones, G. L., Adams, J. M., Anaya, J. M., Bowles, T. J., Chupp, T. E., Coulter, K. P., Dewey, M. S., Freedman, S. J., Fujikawa, B. K., Garcia, A., Greene, G. L., Hwang, S. R., Lising, L. J., Mumm, H. P., Nico, J. S., Robertson, R. G. H., Steiger, T. D., Teasdale, W. A., Thompson, A. K., Wasserman, E. G., Wietfeldt, F. E., Wilkerson, J. F., "Time Reversal in Polarized Neutron Decay: The Emit Experiment," Nucl. Instrum. Meth. A 440 (3), 648 (2000).

Jones, G. L., Gentile, T. R., Thompson, A. K., Chowduri, Z., Dewey, M. S., Snow, W. M., Wietfeldt, F. E., "Test of He-3 Based Neutron Polarizers at NIST," Nucl. Instrum. Meth. A 440 (3), 772 (2000).

Jones, R. L., Kumar, S. K., Ho, D. L., Briber, R. M., Russell, T. P., "Chain Conformation in Ultrathin Polymer Films Using Small Angle Neutron Scattering," Macromol. 34 (3), 559 (2001).

Jung, H. T., Coldren, B., Zasadzinski, J. A., Iampietro, D. J., Kaler, E. W., "The Origins of Stability of Spontaneous Vesicles," in Proceedings of the National Academic Science, (200I) p. 1535.

Kamitakahara, W. A., Trevino, S. F., Buga, S. G., Blank, V. D., "Destructive Annealing of a Superhard Carbon Derived From $\mathrm{C}_{60}$," Phys. Rev. $\mathbf{B}$, in press.

Karlsson, E. B., Abdul-Redah, T., Udovic, T. J., Hjörvarsson, B., Chatzidimitriou-Dreismann, C. A., "Short-Lived Proton Entanglement in Yttrium Hydrides," Appl. Phys. A, in press.

Keimer, B., Casa, D., Ivanov, A., Lynn, J. W., Zimmermann, M. V., Hill, J. P., Gibbs, D., Taguchi, Y., Tokura, Y., "Spin Dynamics and Orbital State in LaTiO ${ }_{3}$,' Phys. Rev. Lett. 85 (18), 3946 (2000).

Kent, M., "A Quantitative Study of Tethered Chains in Various Solution Conditions Using Langmuir Diblock Copolymer Monolayers," Macromol., in press.

Kepa, H., Kutner-Pielaszek, J., Twardowski, A., Sipatov, A. Y., Majkrzak, C. F., Story, T., Galazka, R. R., Giebultowicz, T. M., "Interlayer Correlations in Ferromagnetic Semiconductor Superlattices EuS/PbS," J. Magn. Magn. Mater., in press.

Kessler, E. G., Dewey, M. S., Deslattes, R. D., Henins, A., Borner, H. G., Jentschel, M., Doll, C., Lehmann, H., "The Deuteron Binding and the Neutron Mass," Phys. Lett. A 255 (4-6), 221 (2000).

Kessler, E. G., Dewey, M. S., Deslattes, R. D., Henins, A., Borner, H. G., Jentschel, M., Lehmann, H., "The GAMS4 Flat Crystal Facility," Nucl. Instrum. Meth. A 457 (1-2), 187 (2000).

Khalifah, P., Huang, Q., Ho, D. M.. Zandbergen, H. W., Cava. R. J., " $\mathrm{La}_{7} \mathrm{Ru}_{3} \mathrm{O}_{18}$ and $\mathrm{La}_{4.87} \mathrm{Ru}_{2} \mathrm{O}_{12}$ : Geometric Frustration in Two Closely Related Structures With Isolated $\mathrm{RuO}_{6}$ Octahedra," J. Solid State Chem. 155 (1), 189 (2000). 
Khalifah, P., Jin, R., Liu, Y., Huang, Q., Gao, X. P. A., Ramirez, A. P., Cava, R. J., "Non-Fermi-Liquid Behavior in $\mathrm{La}_{4} \mathrm{Ru}_{6} \mathrm{O}_{19}$ : A Cubic, Non-Perovskite Ruthenate With Metal-Metal Bonding," Nature 411, 669 (2001).

Kilic, C., Ciraci, S., Gulseren, O., Yildirim, T., "Variable and Reversible Quantum Structures on a Single Carbon Nanotube," Phys. Rev. B62 (24), 16345 (2000).

Kim. S. D., Klein, A., Sperling, L. H., Boczar, E. M., Bauer, B. J., "SANS Study of Sulfite End Group Effect on Polystyrene SelfDiffusion," Macromol. 33 (22), 8334 (2000).

Kim, W. G., Chang, M. Y., Garetz, B. A., Newstein, M. C., Balsara, N. P., Lee, J. H., Hahn, H., Patel, S. S., "Effect of Quench Depth on Grain Structure in Quiescently Ordered Block Copolymers," J. Chem. Phys. 114, (22), 10196 (2001).

Kim, W. G., Garetz, B. A., Newstein, M. C., N. P., Balsara, "Maximizing Grain Growth Rate During the Disorder-to-Order Transition in Block Copolymer Melts," J. Polym. Sci. 39 (19), 2231 (2001).

Kim, Y. J., Birgeneau, R. J., Chou, F. C., Erwin, R. W., Kastner, M. A., "Critical Spin Dynamics of the 2D Quantum Heisenberg Antiferromagnets $\mathrm{Sr}_{2} \mathrm{CuO}_{2} \mathrm{C}_{12}$ and $\mathrm{Sr}_{2} \mathrm{Cu}_{3} \mathrm{O}_{4} \mathrm{C}_{12}$," Phys. Rev. Lett. 86 (14), 3144 (2001).

Kim, Y. J., Birgeneau, R. J., Chou, F. C., Greven, M., Kastner, M. A., Lee, Y. S., Wells, B. O., Aharony, A., Entin-Wohlman, O., Korenblit, 1. V., Harris, A. B., Erwin, R. W., Shirane, G., "Neutron Scattering Study of $\mathrm{Sr}_{2} \mathrm{Cu}_{3} \mathrm{O}_{4} \mathrm{C}_{12}$ " Phys. Rev. B64 (2), 4435 (2001).

Kim, Y. J., Wakimoto, S., Shapiro, S. M., Gehring, P. M., Ramirez, A. P., "Neutron Scattering Study of Antiferromagnetic Order in $\mathrm{CaCu}_{3} \mathrm{Ti}_{4} \mathrm{O}_{12}$," Phys. Rev. $\mathbf{B}$, in press.

Kiryukhin, V., Kim, Y. J., Thomas, K. J., Chou, F. C., Erwin, R. W., Huang, Q. Kastner, M. A., Birgeneau, R. J., "Magnetic Properties of the $S=1 / 2$ Quasi-One-Dimensional Antiferromagnet $\mathrm{CaCu}_{2} \mathrm{O}_{3}$," Phys. Rev. B63 (14), 4418 (2001).

Kneller, L. R., Edwards, A. M., Nordgren, C. E., Blasie, J. K., Berk, N. F., Krueger, S., Majkrzak, C. F., "Hydration State of Single Cytochrome Monolayers on Soft lnterfaces Via Neutron Interferometry," Biophys. J. 80 (5), 2248 (2001).

Koehler, R. D., Raghavan, S. R., Kaler, E. W., "Microstructure and Dynamics of Wormlike Micellar Solutions Formed by Mixing Cationic and Anionic Surfactants," J. Phys. Chem. B 104, (47), 11035 (2000)

Koga, T., Shin, K., Zhang, Y., Seo, Y.-S., Rafailovich, M. H., Sokolov, J., Chu, B., Satija, S. K., "Fluctuation-1nduced Swelling of Polymer Thin Films-CO Systems as Gas-Supercritical Transition," J. Phys. Soc. Japan. 70, 347 (2001).

Koga, T., Shin, K., Zhang, Y., Seo, Y.-S., Rafailovich, M. H., Sokolov, J., Chu, B., Satija, S. K., "Polymer Thin Films in Supercritical $\mathrm{CO}_{2}$," in Proceedings of the ASR-2000, J. Phys. Soc. Japan, in press.

Kolb, R., Lin, E. K., Wu, W.-L., Satija, S. K., "Neutron Reflectivity Measurements of Polymer Melt Mobility Near Oxidized Sillicon Surfaces Modified with Self-Assembled Monolayers," Langmuir, in press.

Kondoh, E., Baklanov, M. R., Lin, E. K., Gidley, D. W., Nakashima, A., "Comparitive Study of Pore Size of Low-Dielectric-Constant Porous Spin-on-Glass Films with Different Ways of NonDestructive Instrumentation," J. Appl. Phys., in press.
Koubi, L., Tarek, M., Bandyopadhyay, S., Klein, M. L., "Membrane Structural Perturbations Caused by Anesthetics and NonImmobilizers: A Molecular Dynamics Investigation," Biophys. $\mathrm{J}$. , in press.

Koubi, L., Tarek, M., Bandyopadhyay, S., Klein, M. L., Scharf, D., "Distribution of Non-lmmobilizers and Anesthetics in Model Membranes," Anesthesiology, in press.

Krishnan, K., Almdal, K., Burghardt, W. R., Lodge, T. P., Bates, F. S., "Shear-Induced Nano-Macro Structural Transition in a Polymeric Bicontinuous Microemulsion," Phys. Rev. Lett. 87 (9), 301 (2001).

Krueger, J. K., Gallagher, S. C., Wang, C. L., Trewhella, J., "Calmodulin Remains Extended Upon Binding to Smooth Muscle Caldesmon: A Combined Scattering and Fourier Transform Infrared Spectroscopy Study," Biochem. 39 (14), 3979 (2000).

Krueger, S., "Neutron Reflection From Interfaces With Biological and Biomimetic Materials," Curr. Opin. Coll. Int. Sci. 6 (2), 111 (2001).

Krueger, S., Meuse, C. W., Majkrzak, C. F., Dura, J. A., Berk, N. F., Tarek, M., Plant, A. L., "Investigation of Hybrid Bilayer Membranes With Neutron Reflectometry: Probing the Location of Interactions of Melittin," Langmuir 17 (2), 511 (2001).

Kuznetsov, 1. A., Rudnev, Y. P., Serebrov, A. P., Solovei, V. A., Stepanenko, I. V., Vasiliev, A. V., Mostovoy, Y. A., Zimmer, O. Yerozolimsky, B. G., Dewey, M. S., Wietfeldt, F., "An Experiment to Measure Lambda $=\mathrm{G}(\mathrm{A}) / \mathrm{G}(\mathrm{V})$ From a Combination of Angular Correlation Coefficients in the Beta Decay of Polarized Neutrons," Nucl. Instrum. Meth. A 440 (3), 539, (2000).

Kuznicki, S. M., Bell, V. A., Hillhouse, H. W., Braunbarth, C. M., Jacubinas, R. M., Nair, S., Toby, B. H., Tsapatsis, M., "A Titanosilicate Molecular Sieve with Adjustable Pores for SizeSelective Adsorption of Molecules," Nature 412, 720 (2001).

Lamaze, G. P., Chen-Mayer, H. H., Gerouki, A., and Goldner, R. B., "Analysis of Lithium Transport in Electrochromic Multilayer Films by Neutron Depth Profiling," Surf. Interface Anal. 29, 638 (2000)

La-Orauttapong, D., Noheda, B., Ye, Z.-G., Gehring, P. M., Toulouse, J., Cox, D., Shirane, G., "New Phase Diagram for the Relaxor $\left(\mathrm{Pb}\left(\mathrm{Zn}_{1 / 3} \mathrm{Nb}_{2 / 3}\right) \mathrm{O}_{3-\mathrm{x}} \mathrm{PbTiO}_{3}\right.$," Phys. Rev. $\mathbf{B}$, in press.

Lee, J. H., Balsara, N. P., Krishnamoorti, R., Jeon, H. S., Hammouda, B., "Designing Balanced Surfactants for Mixtures of 1mmiscible Polymers," Macromol. 34, 6557 (2001).

Lee, S.-H., Cheong, S.-W., Yamada, K., Majkrzak, C. F., Aeppli, G., "Charge and Canted Spin Order in $\mathrm{La}_{2-x} \mathrm{Sr}_{x} \mathrm{NiO}_{4}$," Phys. Rev. B63 (6), 0405 (2001).

Lee, S.-H., Qiu, Y., Broholm, C., Ueda, Y., Rush, J. J., "Spin Fluctuations in a Magnetically Frustrated Metal $\mathrm{LiV}_{2} \mathrm{O}_{4}$," Phys. Rev. Lett. 86 (24), 5554 (2001).

Lee, S.-H., Tranquada, J. M., Yamada, K., Buttrey, D. J., Li, Q., Cheong, S.-W., "Freezing of a Stripe Liquid," Phys. Rev. Lett., in press.

Lee, Y., Reisner, B. A., Hanson, J. C., Jones, G. A., Parise, J. B., Corbin, D. R., Toby, B. H., Freitag, A., Larese, J. Z., "New Insight 1nto Cation Relocations Within the Pores of Zeolite Rho: In Situ Synchrotron X-Ray and Neutron Powder Diffraction Studies of Pb- and Cd-Exchanged Rho," J. Phys. Chem. B 105 (30), 7188 (2001). 
Lefebvre, A. A., Lee, J. H., Balsara, N. P., Hammouda, B., "Neutron Scattering From Pressurized Polyolefin Blends Near the Limits of Metastability," Macromol. 33 (21), 7977 (2000).

Leighton, C., Fitzsimmons, M. R., Yashar, P., Hoffman, A., Nogues, J., Dura, J., Majkrzak, C. F., Schuller, I. K., "Two-Stage Magnetization Reversal in Exchange Biased Bilayers," Phys. Rev. Lett. 86 (19), 4394 (2001).

Lenhart, J. L., Van Zanten, J. H., Dunkers, J. P., Parnas, R. S., "Interfacial Response of a Fluorescent Dye Grafted to Glass," Am. Chem. Soc. 16 (21), 8145 (2000).

Lenhart, J. L., Van Zanten, J. H., Dunkers, J. P., Parnas, R. S., "Studying the Buried Interfacial Region With an Immobilized Fluorescence Probe," Macromol. 34 (7), 2225 (2001).

Levin, I., Chan, J. Y., Geyer, R. G., Maslar, J. E., Vanderah, T. A., "Cation Ordering Types and Dielectric Properties in the Complex Perovskite $\mathrm{Ca}\left(\mathrm{Ca}_{1 / 3} \mathrm{Nb}_{2 / 3}\right) \mathrm{O}_{3}$," J. Solid State Chem. 156 (1), 122 (2001).

Levin, I., Chan, J. Y., Maslar, J. E., Vanderah, T. A., Bell, S. M., "Phase Transitions and Microwave Dielectric Properties in the Perovskite-Like $\mathrm{Ca}\left(\mathrm{A}_{10.5} \mathrm{Nb}_{0.5}\right) \mathrm{O}_{3} \mathrm{CaTiO}_{3}$ System," J. Appl. Phys. 90 (2), 904 (2001).

Liao, C., Choi, S. M., Mallamace, F., Chen, S. H., "SANS Study of the Structure and Integration of L64 Triblock Copolymer Micellar Solution in the Critical Region," J. Appl. Crystallogr. 33, 677 (2000).

Licci, F., Gauzzi, A., Marezio, M., Huang, Q., Santoro, A., Masini, R., Bougerol-Chaillout, C., Bordet, P.. "Effects of Re Substitute on the Structure and Superconductivity of $\mathrm{Cu}_{1-x} \operatorname{ReBa}_{2} \mathrm{Cu}_{2} \mathrm{O}$," Physica C 355 (3), 267 (2001).

Likos, C. N., Vaynberg, A., Lowen, H., Wagner, N. J., "Colloidal Stabilization by Adsorbed Gelatin," Langmuir 16 (9), 4100 (2000).

Lin, E. K., Lee, H. J., Bauer, B. J., Wang, H., Wetzel, J. T., Wu, W.-L., "Structure and Property Characterization of Low-k Dielectric Porous Thin Films Determined by X-Ray Reflectivity and SmallAngle Neutron Scattering," "Low Dielectric Constant Materials for IC Applications," edited by P. S. Ho, J. Leu, W.W. Lee, (Springer Publishing Inc.), p. 346.

Lin, E. K., Lee, H. J., Wang, H., Wu, W.-L., "Structure and Property Characterization of Low-k Dielectric Porous Thin Films Determined by X-Ray Reflectivity and Small-Angle Neutron Scattering," in Proceedings of the 2000 International Conference on Characterization and Metrology for ULSI Technology (American Institute of Physics, July 2000), p. 453.

Lin, E. K., Pochan, D. J., Wu, W.-L., Satija, S. K.. "Neutron Reflectivity Measurements of Molecular Mass Effects on Polymer Mobility Near the Polymer/Solid Interface," in Proceedings of the Materials Research Society Meeting (April 2000), p. 256.

Lin, E. K., Soles, C. L., Wu, W.-L., Satija, S. K., Lin, Q., Angelopoulous, M., "Neutron Reflectivity Measurements for the Interfacial Characterization of Polymer Thin Films Photoresists," in Proceedings of the I2th International Conference on Photopolymers," (Society of Plastics Engineers, Brookfield, CT, 2001 ), p. 299.
Lin, E. K., Wu, W.-L., Jin, C., Wetzel, J. T., "Structure and Property Characterization of Porous Low-k Dielectric Constant Thin Films Using X-Ray Reflectivity and Small-Angle Neutron Scattering," in Proceedings of the Materials Research Society Meeting (April 2000), p. 425.

Lin, E. K., Wu, W.-L., Lin, Q. H., Angelopoulos, M., "Feature-Shape and Line-Edge Roughness Measurement of Deep Sub-Micron Lithographic Structures Using Small-Angle Neutron Scattering," Proceeding of the SPIE 26th Annual Internationl Conference on Microlithograply, in press.

Lin, M. Y., Peiffer, D. G., Zhang, Y., Rafailovich, M., "Neutron Scattering Applications in Hydrocarbon Materials," J. Phys. Soc. of Japan. 70, 551 (2001).

Lin, Z., Wu, C., Heller-Zeisler, S., Ondov, J. M., "Chemical Mass Balance of Primary Aerosol Constituents in Baltimore Air," Atmos. Environ, in press.

Lindstrom, R. M., "Limits for Qualitative Detection and Quantitative Determination," edited by, D. R. Lide, A Century of Excellence in Measurements, Standards, and Technology (NIST SP 958), U.S. Govt. Print. Office, Washington, DC, 164-166, 2001.

Ling, X. S., Park, S. R., McClain, B. A., Choi, S. M., Dender, D. C., Lynn, J. W., "Superheating and Supercooling of Vortex Matter in a Nb Single Crystal: Direct Evidence for a Phase Transition at the Peak Effect From Neutron Diffraction," Phys. Rev. Lett. 86 (4), 712 (2001).

Lising, L. J., Hwang, S. R., Adams, J. M., Bowles, T. J., Browne, M. C., Chupp, T. E., Coulter, K. P., Dewey, M. S., Freedman, S. J., Fujikawa, B. K., Garcia, A., Greene, G. L., Jones, G. L., Mumm, H. P., Nico, J. S., Richardson, J. M., Robertson, R. G. H., Steiger, T. D., Teasdale, W. A., Thompson, A. K., Wasserman, E. G., Wietfeldt, F. E., Welsh, R. C., Wilkerson, J. F., "New Limit on the D Coefficient in Polarized Neutron Decay," Am. Phys. Soc. 62 (5), 5501 (2000).

Liu, W. J., Jeng, U., Lin, T. L., Lai, S. H., Shih, M. C., Tsao, C. S., Chiang, L. Y., Sung, L. P., "Adsorption of DodecahydroxylatedFullerene Monolayers at the Air-Water Interface," Physica B 283 (1), 49 (2000).

Livingston, R., Neumann, D. A., Allen, A. J., Fitzgerald, S. A., Berliner, R., "Application of Neutron Scattering to Portland Cement," Neutron News 11 (4), 18 (2000).

Loureiro, S. M., Felser, C., Huang, Q., Cava, R. J., "Refinement of the Crystal Structures of Strontium Cobalt Oxychlorides by Neutron Powder Diffraction," Chem. Mat. 12 (10), 3181 (2000).

Luchette, P. A., Vetman, T. N., Prosser, R. S., Hancock, R. E. W., Nieh, M.-P., Glinka, C. J., Krueger, S., Katsara, S. J., "Morphology of Fast Tumbling Bicelles: A Small Angle Neutron Scattering and NMR Study," Bioch. Et Biophy. Acta 1513 (2), 83 (2001).

Lynch, M. L., Wireko, F., Tarek, M., Klein, M., "Intermolecular Interactions and the Structure of Fatty Acid-Soap Crystals," J. Phys. Chem. B 105, 552 (2001).

Lynn, G. W., Dadmun, M. D., Wu, W.-L., Lin, E. K., Wallace, W. E., "Neutron Reflectivity Studies of the Interface Between a Small Molecule Liquid Crystal, and a Polymer," Liquid Crystals, in press.

Lynn, J. W., "Neutron Studies of Exchange-Coupled Superconductors," in Proceedings of the NATO Advanced Research Workshop (June 13-18th), 14, 121 (2001). 
Lynn, J. W., Canfield, P. C., Hilsher, G., Muller, K. H., Naroozhnyi, V. N., "Progress in Borocarbide Research," in Proceedings of the NATO Conference, 14, 431 (2001).

Lynn, J. W., "Spin Dynamics in the Ferromagnetic Phase of Colossal Magnetoresistive Oxides," J. Supercond. Novel Magn. 13 (2), 263 (2000).

Lynn, J. W., "Magnetic Neutron Scattering," in Methods in Materials Research, edited by E. N., Kaufman, R. Abbaschian, A., Bocarsly, C.-L., Chien, D. Dollimore, B., Doyle, A. Goldman, R., Gronsky, S. Pearton, J. Sanchez, (John Wiley \& Sons, 2000), chap. 13.

Lynn, J. W., Adams, C. P., Mukovskii, Y. M., Arsenov, A. A., Shulyatev, D. A., "Charge Correlations in the Magnetoresistive Oxide $\mathrm{La}_{0.7} \mathrm{Ca}_{0.3} \mathrm{MnO}_{3}$," J. Appl. Phys. 89 (11), 6846 (2001).

Lynn, J. W., Skanthakumar, S., "Neutron Scattering Studies of Lanthanide Magnetic Ordering," in Handbook on the Physics and Chemistry of Rare Earths, edited by K. A. Gschneider, Jr., L. Eyring, and M. B. Maple (Elsevier Science, 2001), p. 315

Lynn, J. W., Huang, Q., Santoro, A., Borchers, J. A., Erwin, R. W., "Magnetic Structure Determinations at NBS/NIST," J. Res. Natl. Inst. Stan., in press.

Magid, L. J., Li, Z., Butler, P. D., "Flexibility of Elongated Sodium Dodecylsulfate Micelles in Aqueous Sodium Chloride: A SmallAngle Neutron Scattering Study," Langmuir 16 (26), 10028 (2000).

Majkrzak, C. F., "Applications of Specular Neutron Reflectometry in Materials Science,” J. Neutron Res. 7, 159 (2000).

Majkrzak, C. F., Berk, N. F., "Advances in Specular Neutron Reflectometry," J. Appl. Phys., in press.

Majkrzak, C. F., Berk, N. F., Krueger, S., Dura, J. A., Tarek, M., Tobias, D., Silin, V., Meuse, C. W., Woodward, J., Plant, A. L., "First-Principles Determination of Hybrid Bilayer Membrane Structure by Phase-Sensitive Neutron Reflectometry," Biophysical J. 79 (6), 3330 (2000).

Majkrzak, C. F., Satija, S. K., Berk, N. F., Krueger, S. K., Borchers, J. A., Dura, J. A., Ivkov, R., O'Donovan, K., "Neutron Reflectometry at the NCNR," Neutron News 12, 25 (2001).

Manley, M. E., Fultz, B., McQueeny, R. J., Brown, C. M., Hults, W. L., Smith, J. L., Thomas, D. J., Osborn, R., Robertson, J. L., "Large Harmonic Softening of the Phonon Density of States of Uranium," Phys. Rev. Lett. 86 (14), 3076 (2001).

Manson, J. L., Huang, Q., Lynn, J. W., Koo, H., Whangbo, M., Bateman, R., Wada, N., Awaga, K., Argyriou, D. N., Miller, J. S., "Long-Range Magnetic Order in $\mathrm{Mn}\left[\mathrm{N}(\mathrm{CN})_{2}\right]_{2}($ pyz) $\{$ pyz $=$ pyrazine $\}$ : Susceptibility, Magnetization, Specific Heat and Neutron Diffraction Measurements and Electronic Structure Calculations," J. Am. Chem. Soc. 123 (1), 162 (2001).

Maranzano, B. J., Wagner, N. J., "The Effects of Interparticle Interactions and Particle Size on Reversible Shear Thickening: HardSphere Colloidal Dispersions," J. Rheol. 45 (5), 1205 (2001).

Maranzano, B. J., Wagner, N. J., "The Effects of Particle Size on Reversible Shear Thickening of Concentrated Colloidal Dispersions," J. Chem. Phys. 114 (23), 10514 (2001).

Maranzano, B. J., Wagner, N. J., Fritz, G., Glatter, O., "Surface Charge of 3-(trimethoxysilyl) Propyl Methacrylate (TPM) Coated Stober Silica Colloids by Zeta-PALS and SANS," Langmuir 16, 10556 (2000).
Martter, T. D., Foster, M. D., Yoo, T., Xu, S., Lizzaraga, G., Quirk, R. P., Butler, P. D., "Determination of the Thermodynamics Interaction Parameter for Blends of Star and Linear Polybutadience," Macromol., in press.

Matejicek, J., Ilavsky, J., Gnäupel-Herold, T., "Neutron Scattering in Studies of Complex Anisotropic Microstructure," in Proceedings 3rd International Conference on Materials Structure and Micromechanics of Fracture, edited by Sandera, P., (Vutium, Brno, CZ, 2001), p. 502.

Matejicek, J., Sampath, S., Gnäupel-Herold, T., Prask, H. J., "Processing Effects on Residual Stress in Sprayed Ni+5\% Al Coatings Comparison of Different Spraying Methods," in Proceedings of the International Thermal Spray Conference, edited by C. C., Berndt, (Materials Park, OH, 2001), p. 351.

Maxelon, M., Pundt, A., Pyckhout-Hintzen, W., Barker, J., Kirchheim, R., "Interaction of Hydrogen and Deuterium With Dislocations in Palladium as Observed by Small Angle Neutron Scattering," Acta Mater. 49 (14), 2625 (2001).

Maxelon, M., Pundt, A., Pyckhout-Hintzen, W., Kirchheim, R., "Small Angle Neutron Scattering of Hydrogen Segregation at Dislocations in Palladium," Scripta Materialia 44 (5), 817 (2001).

McKinley, J. P., Serne, R. J., Zachara, J. M., Schaef, H. T., Zeissler, C. J., Lindstrom, R. M., "The Distribution of Retention of ${ }^{137} \mathrm{Cs}$ in Sediments at the Hanford Site," Environmental Science and Technology, in press.

McKelvey C. A., Kaler, E. W., "Characterization of Nanostructured Hollow Polymer Spheres with Small-Angle Neutron Scattering (SANS)," Colloid and Interface Sci., in press

McKelvey, C. A., Kaler, E. W., Zasadzinski, J. A., Coldren, B., Jung, H. T., "Templating Hollow Polymeric Spheres from Catanionic Equilibrium Vesicles: Synthesis and Characterization," Langmuir 16, 8285 (2000).

McLaughlin, J. C., Neumann, D. A., Gress, D. L., Livingston, R. A., "Non-Destructive Measurement of Freeze/Thaw Cycles in Cement Paste Using Quasi-Elastic Neutron Scattering," in Proceedings of the Third International Conference on Concrete Under Severe Conditions, edited by N. Banthia, K. Sakai, O. E. Gjorv, (The University of British Columbia, Vancouver, Canada, 2001), p.1408.

Michels, A., Weissmuller, J., Wiedenmann, A., Pedersen J. A., Barker, J. G., "Measuring the Exchange-Stiffness Constant of Nanocrystalline Solids by Elastic Small-Angle Neutron Scattering," Phil. Mag. Lett. 80 (12), 785 (2000).

Mildner, D. F. R., "Time Focusing of Pulsed-Source Crystal Analyzer Spectrometers II. Practical Expressions," J. Nuci. Instrum. \& Meth., in press.

Mildner, D. F. R., "Neutron Optics," in The Handbook of Optics III, $X$-Ray and Neutron Optics, edited by W. M. Gibson, C. A. MacDonald, (Optical Society of America, McGraw-Hill Publishing, New York, NY, 2001), p. 36.

Mildner, D. F. R., Carpenter, J. M., "Time Uncertainty for Guided Long Wavelength Neutrons on a Pulsed Source," J. Nucl. Instrum. \& Meth. A, in press.

Mildner, D. F. R., Arif, M., Werner, S. A., "Neutron Transmission Through Pyrolytic Graphite Monochromators," J. Appl. Cryst. 34 (3), 258 (2001). 
Mombru, A. W., Pardo, H., Suescum, L., Toby, B. H., Ortiz, W. A., Negreira, C. A., Araujo-Moreira, F. M., "Influence of Oxygen Disorder on the Magnetic Properties of $\mathrm{LaBaCuFeO}_{5+8}$ : an EXAFS and Neutron Diffraction Study," Physica C 356 (1), 149 (2001).

Mook, H. A., Dai, P., Hayden, S. M., Hiess, A., Lee, S.-H., Dogan F., "Polarized Neutron Beam Observation of Antiferromagnetic Order in $\mathrm{Yba}_{2} \mathrm{Cu}_{3} \mathrm{O}_{6.6}$, Science, in press.

Nair, S., Tsapatsis, M., Toby, B. H.. Kuznicki, S. M., "A Study of Heat-Treatment Induced Framework Contraction in Sr-ETS-4 by Powder Neutron Diffraction and Vibrational Spectroscopy," J. Am. Chem. Soc., in press.

Nakatani, A. I., "Chain Dimensions in Polysilicate-Filled Poly(Dimethylsiloxane)," in Proceedings of the International Journal of Thermophysics, 14th Symposium on Thermophysical Properties, (Boulder, $\mathrm{CO}$ ), in press.

Nakatani, A. I., Chen, W., Schmidt, R. G., Gordon, G. V., Han, C. C., "Chain Dimensions in Polysilicate-Filled

Poly(Dimethylsiloxane)," Polymer 42 (8), 3713 (2001).

Nakatani, A. I., Ivkov, R., Papanek, P., Jackson, C. L., Yang, H., Nikiel, L., Gerspacher, M., Krishnamoorti, R., "The Significance of Percolation in the Dynamics of Polymer Chains Bound to Carbon Black," in Proceedings of the Filled and Nanocomposite Polymer Materials, edited by A. Nakatani, Hjelm, I., Gerpacher, M., Krishnamoorti, R. P., (Materials Research Society, Pittsburgh, 2001), in press.

Nakatani, A. I., Ivkov, R., Papanek, P., Yang, H., Gerspacher, M., "Inelastic Neutron Scattering From Filled Elastomers," Rubber Chem. Technol. 73 (5), 847 (2000).

Nico, J. S., Abdurashitov, J. N., Gavrin, V. N., Girin, S. V., Gorbachev, V. V., Ibragimova, T. V., Kalikhov, A. V., Khairnasov, N. G., Knodel, V. N., Mirmov, I. N., Shikhin, A., Verentenkin, E. P., Vermul, V. M., Yants, V. E., Zatsepin, G. T., Bowles, T. J., Teasdale. W. A., Wark, D. L., Cherry, M. L., Cleveland, B. T., Davis, R., Lande, K., Wildenhain, P. S., Elliott, S. R., Wilkerson, J. F., "Solar Neutrino Results From SAGE," in Proceedings of the 10th International Baksan School: Particles and Cosmology, edited by E. N. Alexeev, V. A. Matveev, K. H. S., Nirov, V. A. Rubakov, (Institute for Nuclear Research of the Russian Academy of Sciences, Moscow, 2000), in press.

Nieh, M.-P., Glinka, C. J., Krueger, S., Prosser, R. S., Katsaras, J., "A SANS Study on the Effect of Lanthanide Ions and Charged Lipids on the Morphology of Phospholipid Mixtures," Biophys. $\mathrm{J}$. , in press.

Nieh, M.-P., Glinka, C. J., Krueger, S., Prosser, R. S., Katsaras, J., "SANS Study of the Structural Phases of Magnetically Alignable Phospholipid Mixtures," Langmuir 17 (9), 2629 (2001).

Nieh, M.-P., Kumar, S., Ho, D., Briber, R. , "Neutron Scattering of Polymer Conformations in Energetic Neutral Pores of the Vycor," Macromol., in press.

Ngai, K. L., Soles, C. L., Bao, L., Yee, A. F., "Correlations of Positron Annihilation and Other Dynamic Properties in Small Molecule Glass-Forming Substances," Phys. Rev. Lett. 87 (21), 901 (2001).

Norman, B. R., Iyengar, G. V., "Further Applications of Pre-Irradiation Combustion and Neutron Activation Analysis Technique for the Determination of Iodine in Food and Environmental Reference Materials: Merits and Demerits," Fresen. J. Anal. Chem., in press.
O’Donovan, K. V., Borchers, J. A., Majkrzak, C. F., Hellwig, O., Fullerton, E. E., "Extracting Buried Twists With Polarized Neutron Reflectometry," Appl. Phys. Lett. A, in press.

O'Donovan, K. V., Borchers, J. A., Majkrzak, C. F., Hellwig, O., Fullerton, E. E., "Pinpointing Chical Structures Using Front/ Back Polarized Neutron Reflectivity," Phys. Rev. Lett., in press.

Ohwada, K., Hirota, K., Rehrig, P. W., Gehring, P. M., Noheda, B., Fujii, Y., EaglePark, S.-E., Shirane, G., "Neutron Diffraction Study of the Irreversible R-MA-MC Phase Transition in Single Crystal $\mathrm{Pb}\left[\left(\mathrm{Zn}_{1 / 3} \mathrm{Nb}_{2 / 3}\right)_{1-x} \mathrm{Ti}_{x}\right] \mathrm{O}_{3}$," J. Phys. Soc. Japan. 70 (9), 429 (2001).

Olson, D. H., Toby, B. H., Reisner, B. A., "Neutron Diffraction Study of Protons in Four Lanthanum Exchanged X and LSX Zeolites," in Proceedings of the 13th International Zeolite Congress, in press.

Ondov, J. M., Caffrey, P. F., Suarez, A. E., Borgoul, P. V., Holsen, T., Paode, R. D., Sofuoglu, S. C., Sivadechathep, J., Lu, J., Kelly, M. J., Church, T., Scudlark, J., "Atmospheric Deposition of Trace Elements to Lake Michigan: Influence of Urban and Industrial South Shore Sources," Environ. Sci. Technol., in press.

Papanek, P., Fischer, J. E., Sauvajol, J. L., Dianoux, A. J., McNeillis, P. M., Mathis, C., Francois, B., "Low-Frequency Phonons in Pristine and Doped Phases of Polyacetylene: An Inelastic Neutron Scattering Study," J. Chem. Phys., in press.

Papanek, P., Kamitakahara, W. A., Zhou, P., Fischer, J. E., "Neutron Scattering Studies of Disordered Carbon Anode Materials," J. Phys. Condens. Mat. 13, 8287 (2001).

Paul, R. L., "Development of a Procedure for Measuring Nitrogen by Cold Neutron Prompt Gamma-Ray Activation Analysis," Analyst 126 (2), 217 (2001).

Paul, R. L., "Determination of Nitrogen in Some Reference Materials by Cold Neutron Prompt Gamma-Ray Activation Analysis," J. Radioanal. Nucl. Chem., in press.

Paul, R. L., "Nitrogen in Some Reference Materials by Cold Neutron Prompt Gamma-Ray Activation Analysis," Analyst, in press.

Paul, R. L., "Nondestructive Measurement of Hydrogen and Other Elements by Cold Neutron Capture Prompt Gamma-Ray Activation Analysis," Geostandards Newsletter, in press.

Paul, R. L., "Reference Minerals for Microanalysis of Light Elements," Geostandards Newsletter, in press.

Paul, R. L., Chen-Mayer, H. H., Lindstrom, R. M., "Characterization of Hydrogen in Concrete by Cold Neutron Prompt Gamma-Ray Activation Analysis and Incoherent Scattering," in Proceedings Nondestructive Methods and Materials Characterization, edited by G. Y. Baaklini, N. Meyendorf, T. E. Matikas, R. S. Gilmore (Matls. Res. Soc., Warrendale, PA, 2000), p.175.

Paul, R. L., Simons, D. S., "Neutron Activation Analysis for Calibration of Phosphorus Implantation Dose," in Proceedings of the Characterization and Metrology for ULSI Technology, edited by D. G. Seiler, A. C. Diebold, W. M. Bullies, T. J. Shaffner, R. McDonald, E. J. Walters (American Institute of Physics, Woodbury, NY, 2001), p. 677.

Payne, A. C., Sprauve, A., Olmstead, M. M., Kauzlarich, S. M., Chan, J. Y., Reisner, B. A., Lynn, J. W., "Synthesis, Magnetic, and Electronic Properties of Single Crystals of EuMn $\mathrm{P}_{2}$," J. Solid State Chem., in press. 
Pelton, R., Richardson, R., Cosgrove, T., Ivkov, R., "The Effects of Temperature and Methanol Concentration on the Properties of Poly(N-Isopropylacrylamide) at the Air-Solution Interface," Langmuir 17 (I6), 5118 (2001).

Perez-Salas, U., Nasser, L., Briber, R. M., Hamilton, W. A., Rafailovich, M. H., Sokolov, J., "Polystyrene Network Interdiffusion," Macromol., in press.

Perez-Salas, U., Briber, R. M., Rafailovich, M. H., Sokolov, J., "Interfacial Fracture Toughness Between Glassy Polymer Networks," J. of Polym. Sci. Part B: Poly. Phys., in press.

Petrash, S., Cregger, T., Zhao, B., Pokidysheva, E., Foster, M. D., Brittain, W. J., Sevastinov, V., Majkrzak, C. F., "Changes in Protein Adsorption on Self-Assembled Monolayers with Monolayer Order: Comparison of Human Serum Albumin and Human Gamma Globulin," Langmuir, in press.

Pivovar, A. M., Ward, M. D., Yildirim, T., Neumann, D. A., "Vibrational Mode Analysis of Isomorphous Hydrogen-Bonded Guanidinium Sulfonates With Inelastic Neutron Scattering and Density Functional Theory," J. Chem. Phys. 115 (4), 1909 (2001).

Plazanet, M., Johnson, M. R., Gale, J. D., Yildirim, T., Kearley, G. J., Trommsdorff, H. P., Fernandez-Diaz, M. T., Sanchez-Portal, D., Artacho, E., Soler, J. M., Ordejon, P., Garcia, A., "The Structure and Dynamics of Crystalline Durene by Neutron Scattering and Numerical Modeling Using Density Functional Methods," Chem. Phys. 261 (1), 189 (2000).

Pochan, D. J., Lin, E. K., Satija, S. K., Wu, W.-L., "Thermal Expansion of Supported Thin Polymer Films: A Direct Comparison of Free Surface vs Total Confinement," Macromol. 34 (9), 3041 (2001).

Pochan, D. J., Lin, E. K., Wu, W.-L., "Surface Directed Crystallization and Autophobic in Semicrystalline Polymer Thin Films," Phys. Rev. Lett., in press.

Pochan, D. J., Lin, E. K., Wu, W.-L., Satija, S. K., Cheng, S. Z. D., "Thermal Expansion and Glass Transition Behavior of Thin Polymer Films With and Without a Free Surface via Neutron Reflectometry," in Proceedings of the Dynawics in Small Confining Systems IV, edited by J. M. Drake, G. S. Grest, J. Klafter, R. Kopelman, MRS Symp. Series 543, (Materials Research Society, Warrendale, PA, 1999), p. 163.

Pochan, D. J., Pakstis, L., Nowak, A. P., Deming, T. J., "SelfAssembled Polypeptide Hydrogels; Morphology and Cytotoxicity," Biomacromol., in press.

Popovici, M., Stoica, A., Hubbard, C., Spooner, S., Prask, H., Gnäupel-Herold, T., Gehring, P., Erwin, R., "Multi-Wafer Focusing Neutron Monochromators and Applications," in Proceedings of the SPIE Optical Science and Teclmology, in press.

Porcar, L., Hamilton, W. A., Butler, P. D., Warr, G. G., "A New Vapor Barrier Couette Shear Cell, for Small Angle Neutron Scattering Measurements," Rev. Sci. Instrum., in press.

Prask. H. J., Brand, P. C., Gnäupel-Herold, T., Hicho, G. E., "Determination of Residual Stresses Using Neutron Diffraction: Tank-Car Welds and Rail Slices," in ASNT's Topics on Nondestructive Evaluation Series, in press.

Prask, H. J., Gnäupel-Herold, T., "Materials Science Using Neutron," in Proceedings Transactions of the American Nuclear Society, American Nuclear Society (Washington, DC, November 12-16, 2000) 83, 495 (2000).
Prask, H. J.. Gnäupel-Herold, T., Fisher, J. W., Cheng, X., Stuart, J. T. "Residual Stress Modification by Means of Ultrasonic Impact Treatment," in Proceedings of the 2001 SEM Annual Conference \& Exposition on Experimental and Applied Mechanics, in press.

Quijada, M. A., Simpson, J. R., Drew, H. D., Lynn, J. W., VasiliuDoloc, L., Mukovskii, Y. M., Karabashev, S. G., "Temperature Dependence of Low-Lying Electronic Excitations of $\mathrm{LaMnO}_{3}$," Phys. Rev. B64, in press.

Raghavan, S. R., Edlund H., Kaler, E. W., "Cloud-Point Phenomena in Wormlike Micellar Systems Containing Cationic Surfactant and Salt," Langmuir, in press.

Raghavan, S. R., Kaler, E. W., "Highly Viscoelastic Wormlike Micellar Solutions Formed by Cationic Surfactants With Long Unsaturated Tails," Langmuir 17, 300 (2001).

Reidy, R. F., Allen, A. J., Krueger, S., "Small Angle Neutron Scattering Characterization of Colloidal and Fractal Aerogels," J. Non Cryst. Solids 285 (1), 181 (2001).

Reisner, B. A., Lee, Y., Hanson, J. C., Jones, G. A., Parise, J. B., Corbin, D. R., Toby, B. H., Freitag, A., Larese, J. Z., Kahlenberg, V., "Understanding Negative Thermal Expansion and Trap Door Cation Relocations in Zeolite Rho," Chem. Comm. 22, 2221 (2000).

Reisner, B. A., Tripathi, A., Parise, J. B., " $\mathrm{Na}_{8}\left[\mathrm{Zn}_{4} \mathrm{Ge}_{8} \mathrm{O}_{18}(\mathrm{OH})_{12}\right] \cdot 2 \mathrm{H}_{2} \mathrm{O}$ : An Interrupted-Framework Zincogermanate Built From $\left[\mathrm{GeO}_{3}(\mathrm{OH})\right]$ and $\left[\mathrm{Ge}_{2}(\mathrm{OH})_{2}\right]$ Units," J. Mater. Chem. 11, 887 (2001).

Rich, D. R., Fan, S., Gentile, T. R., Hussey, D., Jones, G. L., Neff, B., Snow, W. M., Thompson, A. K., "Polarized ${ }^{3}$ He Neutron Spin Filter Development and Application at Indiana University and NIST," Physica B, in press.

Rols, S., Benes, Z., Anglaret, E., Sauvajol, J. L., Papanek, P., Fischer, J. E., Coddens, G., Schober, H., Dianoux, A. J., "Phonon Density of States of Single-Wall Carbon Nanotubes," Phys. Rev. Lett. 85 (24), 5222 (2000).

Rosenkranz, S., Osborn, R., Vasiliu-Doloc, L., Lynn, J. W., Sinha, S. K., Mitchell, J. F., "Observation of Kosterlitz-Thouless Spin Correlations in the Colossally Magnetoresistive Layered Manganite $\mathrm{La}_{1.2} \mathrm{Sr}_{1.8} \mathrm{Mn}_{2} \mathrm{O}_{7}$," Phys. Rev. Lett., in press.

Rossbach, M., Zeisler, R., "Certified Reference Materials for Microanalytical Methods," in Proceedings of the Reference Materials for Chemical Analysis Certification, Availability, and Proper Usage, edited by M. Stoeppller, W. R. Wolf, P. Jenks, (Wiley-VCH Verlag Chemie, Weinmeim, Germany, 2001), p. 127.

Salamat, G., De Vries, R., Kaler, E. W., Satija, S., Sung, L., "Undulations in Salt-Free Charged Lamellar Phases Detected by SmallAngle Neutron Scattering and Neutron Reflectivity," Langmuir 16, I02 (2000).

Sankar, S., Dender, D., Borchers, J. A., Smith, D. J., Erwin, R. W., Kline, S. R., Berkowitz, A. E., "Magnetic Correlations in NonPercolated Co-SiO, Granular Films," J. Magn. Magn. Mater 221 (1), 1 (2000).

Santoro, A., "Neutron Crystallography at NBS/NIST," ACA Newsletter, in press.

Sayer, E. V., Yener, A. K., Joel, E. C., Blackman, M. J., Özbal, H., "Stable Lead lsotope Studies of Black Sea Anatolian Ore Sources and Related Bronze Age and Phrygian Artifacts From Nearby Archaeological Sites Appendix: New Central Taurus Ore Data," Archaeometry, in press. 
Schirber, J. E.. Morosin. B., Kwei, G. H., Yildirim, T. A., Fischer, J. E., Jorgensen, J. D.. "Superconductivity in the Polymeric Phase of $\mathrm{Na}_{2} \mathrm{CsC}_{60}$," Physica C 353, 207 (2001).

Schmidt. G.. Nakatani, A. I., Butler, P. D., Ferreiro, V., Karim, A., Han, C. C., "Polymer-Clay Nanocomposite Materials, Solution and Bulk Properties," in Proceedings of the Materials Research Society Symposium, (2001), p. 20.

Schmidt, G., Nakatani, A. I., Butler, P. D., Karim, A., Han, C. C., "Shear Orientation of Viscoelastic Polymer-Clay Solutions," in Proceedings of the XIIIth International Congress on Rheology (Cambridge, UK, 2000), p. 122.

Schober, H., Losert, C., Mezei, F., Cook, J. C., "Chopper Cascades: An Analytical Treatment of the Contamination Problem." J. Neutron Res. 8, 175 (2000).

Schulz, J. C., Warr, G. G., Butler, P. D., Hamilton, W. A., "Adsorbed Layer Structure of Cationic Surfactants on Quartz," Phys. Rev. E 63 (4), 1604 (2001).

Schwickert, B. E., Kline, S. R., Zimmermann, H., Lantzky, K. M., Yarger, J. L.. "Early Stages of Glacial Clustering in Supercooled Triphenyl Phosphite," Phys. Rev. B64 (4), 5410 (200I).

Sharpless, K. E., Colbert, J. C., Greenberg, R. G., Schantz, M. M., Welch, M. J., "Recent Developments in Good-Matrix Reference Materials at NIST," Fres. J. Anal. Chem. 370, 275 (200I).

Shin, K., Zhang, Y., White, H., Rafailovich, M. H., Sokolov, J., Peiffer, D., "Organoclay Nanoplatelets at the Air/Water Interface," Mat. Res. Soc. Symp, KK8.4 (2001).

Shin, K., Hu, X., Zheng, X., Rafailovich, M. H., Sokolov, J., Zaitsev, V., Schwartz, S. A., "Silicon Oxide Surface as a Substrate for Polymer Thin Films," Macromol. 34 (I4), 4993 (2001).

Shin, K., Pu, Y., Rafailovich, M., Sokolov, J., Seeck, O. H., Tolan, M., Sinha, S., "Corrected Surfaces of Free Standing Polystyrene Thin Films," Macromol. 34 (16), 5620 (2001).

Shin, K., Rafailovich, M. H., Sokolov, J., Change, D. M., Cox. J. K., Lennox, R. B., Eisenberg, A., Huang, J., Hsu, S. L., Satija, S., "Observation of Surface Ordering of Alkyl Side Chains in Polystyrene/Polyelectrolytes Diblock Copolymer Langmuir Films," Langmuir 17 (16). 4955 (2001).

Shin, K., Rafailovich, M., Sokolov, J., Nguyen, D., Yang, N.-L., Eisenburg, A., Satija, S. K., "lonomer Thin Films in Mixed Solvent," Langmuir, 21, 6675 (2001).

Shirane, G., Gehring P. M., "Soft Phonon Anomalies in Relaxor Ferroelectrics." J. Phys. Soc. Jpn. 84 (22). 5216 (2000).

Silas J. A., Kaler, E. W., "Multiple Scattering from Efficient Microemulsions," Colloid and Interface Sci., in press.

Sitepu, H., Prask, H. J., "Use of Generalized Spherical-Harmonic Method for Describing Preferred Orientation With Neutron Powder Diffraction Data," J. Mater. Sci., in press.

Sitepu, H., Prask, H. J., Vaudin, M. D., "Texture Characterization in X-Ray and Neutron Powder Diffraction Data Using the Generalized Spherical-Harmonic," Advances in X-Ray Analysis 44. 241 (2001).

Sitepu, H., Schmahl, W. W., Stalick, J. K., "Use of Generalized Spherical-Harmonic Model for Describing Texture in NiTi Shape Memory Alloy by Neutron Diffraction," Appl. Phys. A, in press.

Skripov, A. V., Cook, J. C., Udovic, T. J., Kozhanov, V. N., "Quasielastic Neutron Scattering Study of Hydrogen Motion in C15-Type HfMo ${ }_{2} \mathrm{H}_{0.26}$ "' Phys. Rev. B62 (21), 14099 (2000).
Skripov, A. V., Cook, J. C., Udovic, T. J., Kozhanov, V. N., Hempelmann, R., "Quasielastic Neutron Scattering Studies of H Motion in Laves-Phase Compounds," Appl. Phys. A. in press.

Smee, S. A., Brand, P. C., Barry, D. D., Broholm, C. L., Anand, D. K., "An Elastic, Low-Background Vertical Focusing Element for a Doubly Focusing Neutron Monochromator," Nucl. Instrum. Methods A 466 (3), 513 (2001).

Smee, S. A., Orndorff, J. D., Scharfstein, G. A., Qiu, Y., Brand, P. C., Broholm, C. L., Anand, D. K., "MACS Low Background Doubly Focusing Neutron Monochromator," Appl. Phys. A, in press.

Snow, W. M., Chowduri, Z., Dewey, M. S., Fei, X., Gilliam, D. M., Greene, G. L., Nico, J. S., Wietfeldt, F. E., "A Measurement of the Neutron Lifetime by Counting Trapped Protons," Nucl. lnstrum. Meth. A 440 (3), 528 (2000)

Sokolov, M. A., Spooner, S. E., Odette, G. R., Wirth, B. D., Lucas, G. E., "SANS Study of High-Copper RPV Welds in Irradiated and Annealed Conditions," in Proceedings of the Effects of Radiation on Materials: 18th Intemational Symposiun, ASTM 1325, edited by R. K. Nanstad, M. L. Hamilton, F. A. Garner, A. S. Kumar (ASTM, West Conshohocken, PA, 1999), p. 333.

Soles, C. L., Dimeo, R. M., Neumann, D. A., Kisliuk, A., Sokolov, A. P., Liu. J., Yee, A. F., Wu, W.-L., "Correlations of the Boson Peak with Annihilation in Series of Polycarbonate Copolymers," Macromol. 34 (12), 4082 (2001).

Soles, C. L., Douglas, J. F., Wu, W.-L., Dimeo, R. M., "Incoherent Neutron Scattering and the Dynamics of Confined Polycarbonate Films," Phys. Rev. E, in press.

Soles, C. L., Douglas, J. F., Wu, W.-L., Penh, H., Gidley, D. W., "A Broad Perspective on Dynamics of Highly Confined Polymer Films," in Proceedings of the MRS (Nov. 2001), in press.

Soles, C. L., Lin, E. K., Lehart. J. L., Jones, R. L., Wu, W.-L., Goldfarb, D., Angelopoulos, M., "Thin Film Confinement Effects on the Thermal Properties of Model Photoresist Polymers," J. Vac. Sci. Technol., in press.

Stancik, C. M., Pople, J. A., Hedrick, J. L., Gast, A. P., "Novel Dendrimer-like Star Copolymer Architectures Investigated With Scattering Techniques, "Polymeric Materials: Science \& Engineering," 85, 127 (2001).

Steinle-Neumann, G., Stixrude, L., Cohen, R. E., Gulseren, O., "Elasticity of Iron at High Pressure and Temperature: Implications for the Earth's 1nner Core," Nature 413, 57 (2001).

Stone, M. B., Rittner, J., Chen, Y., Yardimci, H., Reich, D. H., Broholm, C., Ferraris, D. V., Lectka, T., "Frustrated 3-Dimensional Quantum Spin Liquid in $\mathrm{CuHpCl}$," Phys. Rev. B, in press.

Stone, M. B., Zaliznyak, I. A., D. H., Reich, Broholm, C., "Frustration Induced Quantum Disordered Phase in Two Dimensional Heisenberg Antiferromagnet Piperazinium Hexachlordicuprate," Phys. Rev. B64 144405 (2001).

Streletzky, K. A., Zvinevich, Y., Wyslouzil, B. E., "Controlling Nucleation and Growth of Nanodroplets in Supersonic Nozzles," J. Chem. Phys., in press.

Suarez, A. E., Ondov, J. M., "Atmospheric Deposition of Trace Elements to the Chesapeake Bay: Influence of Urban and Industrial Sources in Baltimore," Environ. Sci. Tech., in press.

Sung, L., Karim, A., Douglas, J. F., Han, C. C., "Modification of Thin Film Phase Separation by a Surfactant," Macromol., in press. 
Tarek, M., Tobias, D. J., "The Dynamics of Protein Hydration Water: A Quantitative Comparison of Molecular Dynamics Simulations and Neutron Scattering Experiments." Biophys. J. 79 (6), 3244 (2000).

Tarek, M., Tobias, D. J., "Effects of Solvent Damping on Side Chain and Backbone Contributions to the Protein Boson Peak," J. Chem. Phys. 115 (3), 1607 (2001).

Terry, J. S., Heller-Zeisler, S., Ondoc, J. M., "Behavior of Three Atmospheric Tracer Materials in a Pilot-Scale Coal Combuster," J. Radio. Analyt. Nuc. Chem., in press.

Thomas, J. J., Neumann, D. A., Fitzgerald, S. A., Livingston, R. A., "The State of Water in Hydrating Tricalcium Silicate and Portland Cement Pastes as Measured by Quasi-Elastic Neutron Scattering," J. Am. Ceram. Soc. 84 (8), 1811 (2001).

Toby, B. H., "The Classification of Powder Diffraction Data," International Tables for Crystallography, in press.

Toby, B. H., "Diffraction Analysis and Crystallographic Structure Determination," Handbook of Ceramic Engineering, edited by M. N. Rahaman, in press.

Toby، B. H., "EXPGU1, a Graphical User 1nterface for GSAS," J. Appl. Crystallgr. 34, 210 (2001).

Toby, B. H., "Structural Studies of Zeolitic Materials With the NIST BT-1 Neutron Powder Diffractometer," Neutron News, 12 (2), 30 (2001).

Trollas, M., Atthof, B., Wursch, A., Hedrick, J. L., Pople, J. A., Gast, A. P., "Constitutional Isomers of Dendrimer-Like Start Polymers: Design, Synthesis, and Conformational and Structural Properties," Macromol. 33, 6423 (2000).

Truong, M. T., Walker, L. M., "Controlling the Shear-Induced Structural Transition of Rodlike Micelles Using Nonionic Polymer," Langumir 16, 7991 (2000).

Tsai, A. M., Neumann, D. A., Bell, L. N., "Molecular Dynamics of Solid-State Lysozyme as Affected by Glycerol and Water: A Neutron Scattering Study," Biophys. J. 79 (5), 2728 (2000).

Tsai. A. M., Udovic, T. J., Neumann, D. A., “The Inverse Relationship Between Protein Dynamics and Thermal Stability," Biophys. J. 81, 2339 (2001).

Tung, C.-S., Wall, M. E., Gallagher, S. C., Trewhella, J., "A Molecular Model of the Troponin C/Troponin Interaction Using Constraints From X-Ray Crystallography, NMR. Small Angle Scattering, Cross-Linking and FRET," Protein Science 78 (1), 2158 (2001).

Udovic, T. J., Karmonik, C., Huang, Q., Rush, J. J., Vennström, M., Andersson, Y., Flanagan, T. B., "Comparison of the Dynamics of Hydrogen and Deuterium Dissolved in Crystalline $\mathrm{Pd}_{9} \mathrm{Si}_{2}$ and $\mathrm{Pd}_{3} \mathrm{P}_{0.8}, " \mathrm{~J}$. Alloy Compnds., in press.

Vajk, O. P., Mang, P. K., Greven, M., Gehring, P. M., Lynn, J. W., "Quantum Impurities in the Two-Dimensional Spin-1/2 Heisenberg Antiferromagnet," Science, in press.

Vasiliu-Doloc, L., Osborn, R., Rosenkranz, S., Mesot, J., Mitchell, J. F., Sinha, S. K., Seeck, O. H., Lynn, J. W., Islam, Z., "Neutron and X-Ray Evidence of Charge Melting in Layered Colossal Magnetoresistance Manganites," J. Appl. Phys. 89 (11), 6840 (2001).

Vasiliu-Doloc, L., Osborn, R., Rosenkranz, S., Mesot, J., Mitchell, J. F., Sinha, S. K., Seeck, O. H., Lynn, J. W., Islam, Z., "Polaron Ordering in Ferromagnetic Colossal Magnetoresistive Oxides," Int. J. Mod. Phys. B 14 (29), 3711 (2000).
Wada, N., Kamitakahara, W. A., "Quasielastic and Inelastic Neutron Scattering Studies on Intercalated Water Molecules in Zeolite LTA and FAU," Mol. Cryst. Liq. Cryst., in press.

Wagner, N. J., Butera, R., "Shear Distortion and Relaxation Dynamics of Colloidal Crystals Investigated by SANS Time Slicing" Phys. Rev. E, in press.

Wakimoto, S., Birgeneau, R. J., Lee, Y. S., Shirane, G., "Hole Concentration Dependence of the Magnetic Moment in Superconducting and lnsulating $\mathrm{La}_{2-x} \mathrm{Sr}_{x} \mathrm{CuO}_{4}$," Phys. Rev. $\mathrm{B} 63$ (17), 2501 (2001).

Wakimoto, S., Tranquada, J. M., Ono, T., Kojima, K. M., Uchida, S., Lee, S.-H., Gehring, P. M., Birgeneau, R. J., "Diagonal Static Spin Correlations in the Low Temperature Orthorhombic Pecn Phase of $\mathrm{La}_{1.55} \mathrm{Nd}_{0.4} \mathrm{Sr}_{0.05} \mathrm{CuO}_{4}$," Phys. Rev. B, in press.

Walker, L. M., Truong, M. T., "Rheology and Structural Transitions in Wormlike Micellar Systems Controlled by Added Nonionic Polymer," in Proceedings of the XIIIth International Congress on Rheology (Cambridge, UK 2000), p.310.

Wall, M. E., Gallagher, S. C., Trewhella, J., "Large-Scale Shape Changes in Proteins and Macromolecular Complexes" Annual Reviews of Physical Chemistry 51, 335 (2000).

Wang, H. Douglas, J. F., Satija, S. K., Composto, R. J., Han. C. C., "Early-Stage Compositional Segregation in Polymer Blend Films," Phys. Rev. Lett., in press.

Wang, H. Y., Hobbie, E. K., Han, C. C., Composto, R. J., "Multiple Lateral Scales in Phase-Separating Thin Films Polymer Blends" Langmuir, 17, 2857 (2001).

Wang, H. Y., Hwang, S. R., Li, W.-H., Lee, K. C., Lynn, J. W., Luo, H. M., Ku, H. C., "Variation in the Structural and Magnetic Properties Induced by La-Doping in $\operatorname{Pr}\left(\mathrm{Ba}_{1-x} \mathrm{La}_{x}\right)_{2} \mathrm{Cu}_{3} \mathrm{O}_{7+y}$," Phys. Rev. B62 (17), 11549 (2000).

Weissmüller, J., Michels, A., Barker, J. G., Wiedenmann, A., Pedersen, J. S., Erb, U., Shull, R. D., "Analysis of Small-Angle Neutron Scattering of Nanocrystalline Ferromagnets by a Micromagnetics Model," Phys. Rev. B63 (21), 4414 (2001).

Welp, K. A., Co, C., Wool, R. P., "Improved Reflectivity Fitting Using SERF: Spreadsheet Environment Reflectivity Fitting," J. Neutron Res., in press.

Wesley, R., Armes, S. P., Thompson, L. J., "Block Copolymer Interactions With Surfactants," Langmuir, in press.

Wesley, R. D., Cosgrove, T., Thompson, L., Armes, S. P., Billingham, N., "The Structure of Hydrophilic-Hydrophobic Block Copolymers and Their Interactions With Salt and a Surfactant," in press.

Williams, R. E., "Recent Advances in Cold Neutron Utilization at the NIST Research Reactor," Trans. Am. Nucl. Soc. 84, 124 (2001).

Williams, R. E., Rowe, J. M., "Developments in Neutron Beam Devices and an Advanced Cold Source for the N1ST Research Reactor," Physica B, in press.

Wirth, B. D., Odette, G. R., Pavinich, W. A., Lucas, G. E., Spooner, S. E., "Small Angle Neutron Scattering Study of Linde 80 RPV Welds," in Proceedings of the Effects of Radiation on Materials: 18th International Symposium, ASTM STP 1325, edited by R. K. Nanstad, M. L. Hamilton, F. A. Garner, and A. S. Kumar (West Conshohocken, PA, 1999), p. 102.

Wiyatno, W., Pople, J. A., Gast, A. P., Waymouth, R., Fuller, G. G., "A Scattering Study of Elastomeric Polypropylene," Macromol., in press. 
Wong-Ng, W., Huang, Q., Lavin, I., Kaduk, J. A., Dillingham, J., Huagan. T., Suh, J., Cook, L. P., "Crystal Chemistry and Phase Equilibria of Selected $\mathrm{SrO}-\mathrm{R}_{2} \mathrm{O}_{3}-\mathrm{CuO}_{x}$ and Related Systems, $\mathrm{R}=$ Lanthanides and Yttrium," J. Solid State Chem., in press.

Wong-Ng, W., Kaduk, J. A., Huang, Q., Roth, R. S., "Crystal Structure of the Monoclinic Perovskite $\mathrm{Sr}_{3.94} \mathrm{Ca}_{1.31} \mathrm{Bi}_{2.70} \mathrm{O}_{12}$," Powder Diff. 15 (4), 227 (2000).

Wu. W.-L., Lin. E. K., Jin, C., Wetzel, J. T. A., "A Three-Phase Model for the Structure of Porous Thin Films Determined by X-Ray Reflectivity and Small Angle Neutron Scattering," in Proceedings of the Materials Teclinology, and Reliability for Advanced Interconnects and Low-k Dielectrics, edited by K. Maex, Y.-C. Joo, G. S., Oehrlein, S. Ogawa, J. T. Wetzel (Materials Research Society, San Francisco. CA, 2001), p. 612.

Wu, W.-L., Lin, E. K., Lin, Q., Angelopolous, M., "Small-Angle Neutron Scattering Measurements for the Characterization of Lithographically Prepared Structures," in Proceedings of the 2000 International Conference on Characterization and Metrology for ULSI Technology, (2000), p. 357.

Wu, W.-L., Lin, E. K., Lin, Q., Angelopolous, M., "Small Angle Neutron Scattering Measurements of Nanoscale Lithographic Features," J. Appl. Phys. 88 (12), 7298 (2000).

Wyslouzil. B. E., Wilemski, G., Strey, R., "Aerosol SANS: A New Method to Probe the Structure of Nanodroplets," in Proceedings of Nucleation and Atmospheric Aerosols 2000, edited by Hale, B. H., Kulmala, M., (American Institute of Physics, New York, 2000), p. 724.

Xu, G., Broholm, C., Reich, D. H., Adams, M. A., "Triplet Waves in Quantum Spin Liquid," Phys. Rev. Lett., in press.

Xu, P., Qu. S., Rosenmayer, T., Lin, M. Y., “High-Temperature Mechanical Behavior and Phase Morphology of Poly(tetrafluoroethylene)/Siloxane Nanocomposites Used as Ultra Low-k Dielectrics," in Proceedings of Materials, Technology, and Reliability for Advanced Interconnects and Low-k Dielectrics, edited by K. Maex, Y.-C., Joo, G. S., Oehrlein, S. Ogawa, J. T. Wetzel (Materials Research Society, Warrendal, P. A., 2000), p. 292.

Yamaura, K., Huang, Q., Takayama-Muromachi, E., "Crystal Structure and Magnetism of the Linear-Chain Copper Oxides $\mathrm{Sr}_{5} \mathrm{~Pb}_{3-\mathrm{x}} \mathrm{Bi}_{x} \mathrm{CuO}_{12}$ " Phys. Rev. B, in press.

Yang. G. Y., Briber, R. M., Huang, E., Rice, P. M., Volksen, W., Miller, R. D., "Formation and Characterization of Ultra-Low Dielectric Materials Using TEM and SANS," in Proceedings of the American Chemical Society, Polymeric Materials: Science and Engineering 85, 18 (2001).

Yang, G. Y., Briber, R. M., Huang, E., Rice, P. M., Volksen, W., Miller, R. D., "Morphological Development in Nanoporous PMSSQ Films," Chem. Mater., in press.

Yang, G. Y., Briber, R. M.. Huang, E., Rice, P. M., Volksen, W., Miller, R. D., "Morphology of Nanoporous PMSSQ Films," Appl. Phys. Lett., in press.

Yang, H., Al-Brithen, H., Smith, A. R., Borchers, J. A., Cappelletti, R. L., Vaudin. M. D., "'Structural and Magnetic Properties of $\eta$-phase Manganese Nitride Films Grown by Molecular Beam Epitaxy," App. Phys. Lett. 78 (24), 3860 (2001).

Yang, L., Harroun, T. A., Weiss T. M., Ding, L., Huang, H. W., "Barrel-Stave Model or Toroidal Model? A Case Study of Melittin Pores," Biophys. J. 81, 1475 (2001).
Yang, S., Mirau, P., Pai, C. S., Nalamasu, O., Reichmanis, E., Lin, E. K., Lee, H. J. Gidley, D., Frieze, W., Dull, T., Sun, J., Yee, A. F.. "Design of Nanoporous Ultra Low-Dielectric Constant Organosilicates by Self-Assembly," in Proceedings of the ACS National Meeting 2001, Polymeric Materials: Science and Engineering, in press.

Yang, S., Mirau, P., Pai, C. S., Nalamasu, O., Reichmanis, E., Pail, J. C., Obeng, Y. S., Seputro, J., Lin, E. K., Lee, H. J., Sun, J. Gidley, D. W., "Nanoporous Ultra Low-Dielectric Constant Organosilicates Templated by Triblock Copolymers," Chem. Mat., in press.

Yang, S., Mirau, P., Pai, C. S., Nalamasu, O., Reichmanis, E., Lin, E. K., Lee, H. J. Gidley, D. W., Sun, J., "Molecular Templating of Nanoporous Ultra Low-Dielectric-Constant $(\sim 1.5)$ Organosilicates by Tailoring the Microphase Separations of Triblock Copolymers," Chem. Mat., in press.

Yang. S., Pai, C. S., Nalamasu, O., Reichmanis, E., Mirau, P., Obeng, Y. S., Seputro, J., Lin, E. K., Lee, H. J., "Design of a Nanoporous Ultra Low-Dielectric Constant Organosilicate," in Proceedings of the 7th International Conference of Polymers in Electronic Packing, in press.

Yang, L., Weiss, T. M., Huang, H. W., "Crystallization of Antimicrobial Pores in Membranes: Magainin and Protegrin," Biophys J. 79 (4), 2002 (2000).

Yildirim, T., "Structure and Dynamics From Combined Neutron Scattering and First-Principles Studies," Chem. Phys. 261 (1), 205 (2000).

Yildirim, T., Gehring, P. M., Neumann, D. A., "The Dynamics of Solid Cubane," Neutron News 12 (2), 18 (2001).

Yildirim, T., Gulseren. O., "A Simple Theory of $40 \mathrm{~K}$ Superconductivity in $\mathrm{MgB}_{2}$; First Principles Calculations of $\mathrm{T}_{c}$, its Dependence in Boron Mass and Pressure," J. Phys. Chem. Solids, in press.

Yildirim, T., Gulseren, O., "First-Principles Zone-Center Theory of Superconductivity in $\mathrm{MgB}_{2}$, " Physica A, in press.

Yildirim, T., Gulseren, O., Ciraci, S., "Exohydrogenated Single-Wall Carbon Nanotubes," Phys. Rev. B64 (7), 5404 (2001).

Yildirim, T., Gulseren, O., Ciraci, S., Kilic, C., "Pressure-Induced Interlinking of Carbon Nanotubes," Phys. Rev. B62 (19), 12648 (2000).

Yildirim, T., Gulseren, O., Lynn, J. W., Brown, C. M., Udovic, T. J., Huang, Q. Z., Rogado, N., Regan, K. A., Sluksy, J. S., He, T., Haas, M. K., Khalifah, P., Inumaru, K., Cava, R. J., "Giant Anharmonicity and Non-Linear Electron-Phonon Coupling in $\mathrm{MgB}_{2}$; Combined First-Principles Calculations and Neutron Scattering Study," Phys. Rev. Lett. 87 (3), 7001 (2001).

Yildirim, T., Reisner, B., Udovic, T. J., Neumann, D. A., "The Combined Neutron Scattering and First-Principles Study of Solid State Protonic Conductors," Solid State Ionics, 145, 429 (2001).

Yong, G., Toulouse, J., Erwin R., Shapiro, S. M., Hennion, B., "Pretransitional Diffuse Neutron Scattering in the Mixed Perovskite Relaxor $\mathrm{K}_{1-x} \mathrm{Li}_{\mathrm{x}} \mathrm{TaO}_{3}$," Phys. Rev. B62 (22), 14736 (2000).

Young, S. K., Trevino, S. F., Beck Tan, N. C.," Small Angle Neutron Scattering Investigation of Structural Changes in Nafion Membranes Induced by Swelling with Various Solvents," J. Polym. Sci. Part B: Polym. Phys. in press. 
Young, S. K., Trevino, S. F., Beck Tan, N. C., Paul, R. L., "Utilization of Prompt Gamma Neutron Activation Analysis in the Evaluation of Various Counterion Nafion Membranes," J. Polym. Sci. Part B: Polym. Phys., in press.

Yun, S. 1., Briber, R. M., Kee, R. A., Gauthier, M., "Small Angle Neutron Scattering of Arborecent Graft Poly(styrene)-Poly(2vinylpyridine) Polymers," in Proceedings of the American Chemical Society, Polymeric Materials: Science and Engineering 85, 224 (2001).

Zaliznyak, I. A., Hill, J. P., Tranquada, J. M., Erwin, R., Moritomo, Y., "Independent Freezing of Charge and Spin Dynamics in $\mathrm{La}_{1.5} \mathrm{Sr}_{0.5} \mathrm{CoO}_{4}$," Phys. Rev. Lett. 85 (20), 4353 (2000).

Zaliznyak, I. A., Lee, S.-H., Petrov, S. V., "Continum in the Spin Excitation Spectrum of a Haldane Chain, Observed by Neutron Scattering in $\mathrm{CsNiCl}_{3}$ " Phys. Rev. Lett. 87 (1), 7202 (2001).

Zamlynny, V., Burgess, I., Szymanski, G., Lipkowski, J., Majewski, J., Smith, G., Satija, S., Ivkov, R., "Electrochemical and Neutron Reflectivity Studies of Spontaneously Formed Amphiphilic Surfactant Bilayers at the Gold-Solution Interface," Langmuir 16 (25), 9861 (2000).

Zeisler, R., Jenks, P., "Proper Usage of Reference Materials," in Proceedings of the Reference Materials for Chemical AnalysisCertification, Availability, and Proper Usage, edited by M. Stoeppller, W. R. Wolf, P. Jenks (Wiley-VCH Verlag Chemie, Weinheim, Germany, 2001), p. 236.

Zeisler, R., Lamaze, G. P., Chen-Mayer, H. H., "Coincidence and AntiCoincidence Measurements in Prompt Gamma Neutron Activation Analysis With Pulsed Cold Neutron Beams," J. Radioanal. Nucl. Ch. 248 (1), 35 (2001).

Zeissler, C. J., Lindstrom, R. M., McKinley, J. P., "Radioactive Particle Analysis by Digital Autoradiography," J. Radioanal. Nucl. Chem. 248, 407 (2001).

Zhang, Y., Douglas, J. F., Ermi, B. D., Amis, E. J., "Influence of Counterion Valcncy on the Scattering Properties of Highly Charged Polyelectrolyte Solutions," J. Chem. Phys. 114 (7), 3299 (2001).

Zhang, Y. M., Ge, S., Tang, B., Rafailovich, M. H., Sokolov, J. C., Peiffer, D. G., Li, Z., Dias, A. J., McElrath, K. O., Satija, S. K., Lin, M. Y., Nguyen, D., "Reduced Mobility of Elastomers Near A Solid Surface as Measured by Neutron Reflectometry," Langmuir 17 (14), 4437 (2001).

Zhang, Y., Ge, S., Tang, B., Rafailovich, M. H., Sokolov, J., Peiffer, D., Li, Z., Lin, M., Dias, J. A., McElrath, K. O., Nguyen, D., Satija, S., Schwarz, S. A., "Interfacial Properties of Brominated Isobutylene-co-p-Methylstyrene and Butadiene Polymers," in Proceedings of the American Chemical Society 154th Rubber Conference, in press.

Zhang, Y., Ge, S., Tang, B., Koga, T., Rafailovich, M. H., Sokolov, J. C., Peiffer, D. G., Li, Z., Dias, A. J., McElrath, K. O., Lin, M.Y., Satija, S. K., Urquhart, S. G., Ade, H., Nguyen, D., "Effect of Carbon Black and Silica Fillers in Elastomer Blends," Macromol. 34, 7056 (2001).

Zhang, Y., Ge, S., Tang, B., Rafailovich, M. H., Sokolov, J. C., Peiffer, D. G., Li, Z., Dias, A. J., McElrath, K. O., Satija, S. K., Lin, M. Y., Nguyen, D., "Reduced Mobility of Elastomers Near" a Solid Surface as Measured by Neutron Reflectometry," Langmuir 17, 4437 (2001).
Zhang, Y., Li, W., Tang, B., Ge, S., Hu, X., Rafailovich, M. H., Sokolov, J. C., Gersappe, D., Peiffer, D. G., Li, Z., Dias, A. J., McElrath, K. O., Lin, M. Y., Satija, S. K., Urquhart, S. G., Ade, H., "Interfacial Properties of Elastomer Blends as Studied by Neutron Reflectivity," Polymer 42, 9133 (2001).

Zhang, Y., Rafailovich, M. H., Sokolov, J. C., Lin. M. Y., Peiffer, D. G., "Interfacial and Nanostructural Studies of Polymer Blends," in Proceedings $8^{\text {th }}$ Asia Pacific Physics Conference, edited by Y. D., Yao, H.-Y. Cheng, C.-S., Chang, Lee, S. F., (World Scientific, Singapore, 2001), p. 292.

Zheludev, A., Chen, Y., Broholm, C. L., Honda, Z., Katsumata, K., "Haldane-Gap Excitations in the Low-H-c One-Dimensional Quantum Antiferromagnet $\mathrm{Ni}\left(\mathrm{C}_{5} \mathrm{D}_{14} \mathrm{~N}_{2}\right)_{2} \mathrm{~N}-3\left(\mathrm{PF}_{6}\right)$," Phys. Rev. B63 (10), 4410 (2001).

Zheludev, A., Honda, Z., Chen, Y., Broholm, C. L., Katsumata, K., Shapiro, S. M., "Gapless Excitations in the High-Field Phase of a Haldane Antiferromagnet," Phys. Rev. Lett., in press.

Zheludev, A., Maslov, S., Zaliznyak, I., Regnault, L. P., Masuda, T., Uchinokura, K., Erwin, R., Shirane, G., "Experimental Evidence for Shekbtman-Entin-Wohlman-Aharony (SEA) Interactions in $\mathrm{Ba}_{2} \mathrm{CuGe}_{2} \mathrm{O}_{7}$," Phys. Rev. $\mathbf{B}$, in press.

Zheludev, A., Masuda, T., Tsukada, I., Uchiyanna, Y., Uchinokura, K., Boni, P., Lee, S.-H., "Magnetic Excitations in Coupled Haldane Spin Chains Near the Quantum Critical Point," Phys. Rev. B62 (13), 8921 (2000).

Zhokhov, A. A., Emelchenko, G. A., Naumenko, I. G., Barilo, S. N., Khasanov, S. S., Zverkov, S. A., Lynn, J. W., "Growth of Large $\mathrm{PrBa}_{2} \mathrm{Cu}_{3} \mathrm{O}_{7-\delta}$ Single Crystals by Modified TSSG Method Using Zirconium Dioxide Crucibles," J. Cryst. Growth 219 (4), 385 (2000).

Zhou, C., Hobbie, E. K., Bauer, B. J., Han, C. C., "Equilibrium Structure of Hydrogen-Bonded Polymer Blends," J. Polym. Sci. Part B: Poly. Phys., in press.

Zimmerman, M. H., Baskin, D. M., Faber, K. T., Fuller, E. R., Allen, A. J., Keane, D. T., "Fracture of Textured Iron Titanate," Acta. Mater. 49, 3231 (2001).

Zimmerman, M. V., "X-Ray Scattering Studies of Orbital and Charge Ordering in $\operatorname{Pr}_{1-x} \mathrm{Ca}_{x} \mathrm{MnO}_{3}$," Phys. Rev. B, in press. 


\section{Instruments and Contacts}

High resolution powder diffractometer $(B T-1)$

J. K. Stalick, (301) 975-6223, judy.stalick@ nist.gov

B. H. Toby, (301) 975-4297, brian.toby@ nist.gov

DARTS, Residual stress and texture diffractometer (BT-8)

H. J. Prask, (301) 975-6226, hank@ nist.gov

T. Gnaeupel-Herold, (301) 975-5380, thomas.gnaeupel-herold@ nist.gov

30-m SANS instrument (NG-7)

C. J. Glinka, (301) 975-6242, cglinka@ nist.gov

J. B. Barker, (301) 975-6732, barker@ nist.gov

B. S. Greenwald, (301) 975-5795, bsgreen@nist.gov

30-m SANS instrument (NG-3) (NIST/NSF-CHRNS)

B. Hammouda, (301) 975-3961, hammouda@ nist.gov

S. R. Kline, (301) 975-6243, steven.kline@ nist.gov

D. Ho, (301) 975-6422, derek.ho@ nist.gov

T. K. Misra, (301) 975-6650, tmisra@ nist.gov

8-m SANS instrument (NG-1)

D. Ho, (301) 975-6422, derek.ho@ nist.gov

C. J. Glinka, (301) 975-6242, cglinka@ nist.gov

J. G. Barker, (301) 975-6732, john.barker@ nist.gov

USANS, Perfect crystal SANS (BT-5) (NIST/NSF-CHRNS)

J. B. Barker, (301) 975-6732, barker@ nist.gov

C. J. Glinka, (301) 975-6242, cglinka@ nist.gov

Cold neutron reflectometer-vertical sample-polarized beam option (NG-1)

C. F. Majkrzak, (301) 975-5251, cmajkrzak@nist.gov

J. A. Dura, (301) 975-6251, jdura@ nist.gov

Cold neutron reflectometer-horizontal sample (NG-7)

S. K. Satija, (301) 975-5250, satija@nist.gov

K.W. Shin, (301) 975-8829, kwshin@nist.gov

Triple-axis polarized-beam spectrometer (BT-2)

J.W. Lynn, (301) 975-6246, jeff.lynn@ nist.gov

Triple-axis fixed incident energy spectrometer (BT-7)

J.W. Lynn, (301) 975-6246, jeff.lynn@ nist.gov

Triple-axis spectrometer (BT-9)

R. W. Erwin, (301) 975-6245, rerwin@ nist.gov

P. M. Gehring, (301) 975-3946, pgehring@ nist.gov

SPINS, Spin-polarized triple-axis spectrometer (NG-5)

(NIST/NSF-CHRNS)

S. Park, (301) 975-8369, sungil.park@ nist.gov

P. M. Gehring, (301) 975-3946, pgehring@ nist.gov

S.-H. Lee, (301) 975-4257, seung-hun.lee@nist.gov
FANS, Filter-analyzer neutron spectrometer (BT-4)

T. J. Udovic, (301) 975-6241, udovic@ nist.gov

C. M. Brown, (301) 975-5134, craig.brown@ nist.gov

D. A. Neumann, (301) 975-5252, dan@ nist.gov

FCS, Fermi-chopper time-of-flight spectrometer (NG-6)

C. M. Brown, (301) 975-5134, craig.brown@ nist.gov

T. J. Udovic, (301) 975-6241, udovic@ nist.gov

DCS, Disk-chopper time-of-flight spectrometer (NG-4) (NIST/NSF-CHRNS)

J. R. D. Copley, (301) 975-5133, jcopley@ nist.gov

J. C. Cook, (301) 975-6403, jeremy.cook@ nist.gov

HFBS, High-flux backscattering spectrometer (NG-2) (NIST/NSF-CHRNS)

R. M. Dimeo, (301) 975-8135, robert.dimeo@ nist.gov

Z. Chowdhuri, (301) 975-4404, zema.chowdhuri@ nist.gov

D. A. Neumann, (301) 975-5252, dan@ nist.gov

NSE, Neutron spin echo spectrometer (NG-5) (NIST/NSF-CHRNS)

N. S. Rosov, (301) 975-5254, nrosov@ nist.gov

Prompt-gamma neutron activation analysis (NG-7)

R. M. Lindstrom, (301) 975-6281, dick.lindstrom@ nist.gov

R. L. Paul, (301) 975-6287, rpaul@nist.gov

Other activation analysis facilities

R. R. Greenberg, (301) 975-6285, rgreenberg@ nist.gov

Cold neutron depth profiling (NG-0)

G. Lamaze, (301) 975-6202, lamaze@ nist.gov

Instrument development station (NG-0)

D. F. R. Mildner, (301) 975-6366, mildner@ nist.gov

H. H. Chen, (301) 975-3782, chenmayer@ nist.gov

Neutron interferometer (NG-7)

M. Arif, (301) 975-6303, muhammad.arif@ nist.gov

Fundamental neutron physics station (NG-6)

M. S. Dewey, (301) 975-4843, mdewey@ nist.gov

Theory and modelling

N. F. Berk, (301) 975-6224, nfb@ nist.gov

T. Yildirim, (301) 975-6228, taner@ nist.gov

Sample environment

D. C. Dender, (301) 975-6225, dender@ nist.gov 


\section{NIST Center for Neutron Research}

For copies of or information on this report, contact:

Ronald L. Cappelletti

(301) 975-6221

ron.cappelletti@nist.gov

For additional information on the facility, contact:

\section{J. Michael Rowe}

(301) 975-6210

mike.rowe@nist.gov

John J. Rush

(301) 975-6231

john.rush@nist.gov

To obtain guidelines for preparing proposals to conduct research at the facility, contact:

William Kamitakahara

(301) 975-6878

william.kamitakahara@nist.gov

Location of all contacts:

NIST Center for Neutron Research

Building 235, Room E151, Mail Stop 8562

National Institute of Standards and Technology

Gaithersburg, MD 20899-8562

Details are also available on the

NCNR Web site:

www.nenr.nist.gov. 
